\title{
Role of Distal Regulatory Elements in Cancer Progression and Therapy
}

\author{
Dissertation \\ for the award of the degree \\ "Doctor of Philosophy (Ph.D)" \\ within the doctoral program Molecular Medicine \\ of the Georg-August University School of Science (GAUSS) \\ at the Georg-August-Universität Göttingen
}

Faculty of Medicine

submitted by

Feda' Hisham Moh'd Hamdan

born in Amman, Jordan

Göttingen, 2018 


\section{Thesis Committee}

Prof. Dr. Steven A. Johnsen, Department for General, Visceral and Pediatric Surgery, University Medical Center, Göttingen

Prof. Dr. med. Volker Ellenrieder, Department of Gastroenterology and Gastrointestinal Oncology, University Medical Center, Göttingen

Prof. Dr. Heidi Hahn, Department of Human Genetics, University Medical Center, Göttingen

\section{Members of the Examination Board}

$1^{\text {st }}$ Referee: Prof. Dr. Steven A. Johnsen, Department for General, Visceral and Pediatric Surgery, University Medical Center, Göttingen

$2^{\text {nd }}$ Referee: Prof. Dr. Volker Ellenrieder, Department of Gastroenterology, University Medical Center, Göttingen

\section{Further members of the Examination Board}

Prof. Dr. Heidi Hahn, Department of Human Genetics, University Medical Center, Göttingen

Prof. Dr. Matthias Dobbelstein, Department of Molecular Oncology, University Medical Center, Göttingen

Dr. Nico Posnien, Department of Developmental Biology, Georg-August-University Göttingen

Dr. Ufuk Günesdogan, Department of Developmental Biology, Georg-August-University Göttingen

Date of oral examination: $12^{\text {th }}$ of December, 2018 


\section{Affidavit}

I hereby declare that the PhD thesis entitled "Role of Distal Regulatory Elements in Cancer Progression and Therapy" has been written independently and with no other sources and aids than quoted.

Feda Hamdan

October, 2018

Göttingen 


\section{Table of Contents}

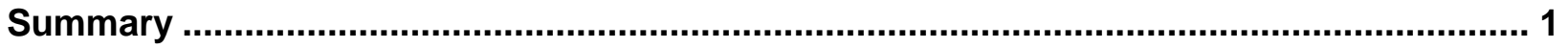

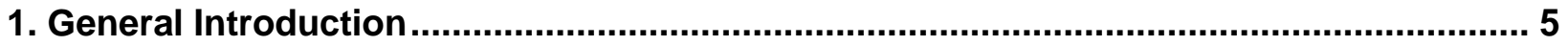

1.1. Epigenetic regulation of gene transcription ……........................................................ 5

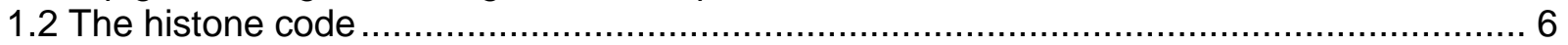

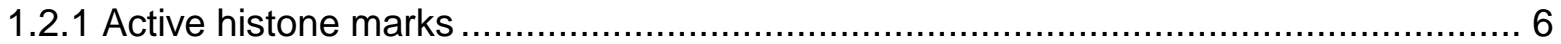

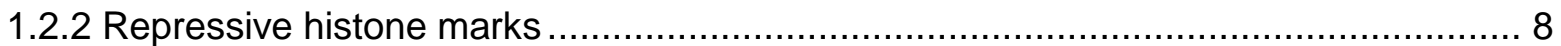

1.2.3 The epigenetic machinery regulating the histone code ……................................. 9

1.2.4 Histone marks signify distinct elements throughout the genome ............................11

1.3 Enhancers and gene transcription ...................................................................

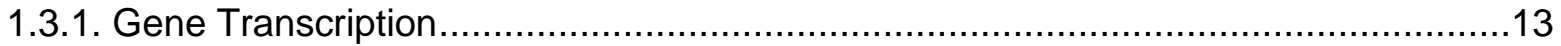

1.3.2 Distal regulatory elements (Enhancers) ……….........................................15

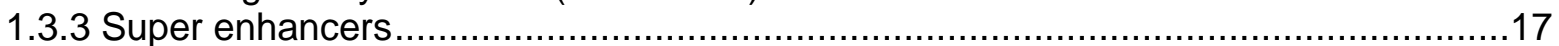

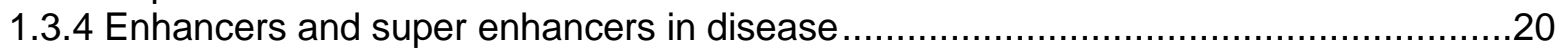

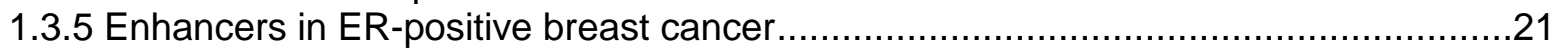

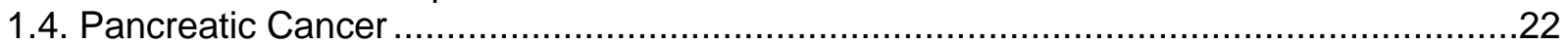

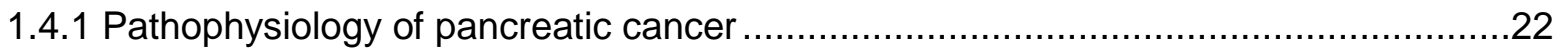

1.4.2 Therapeutic management of pancreatic cancer ...............................................2

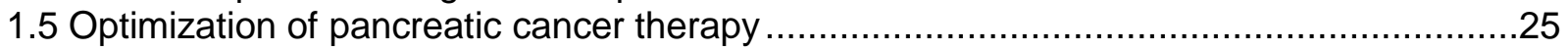

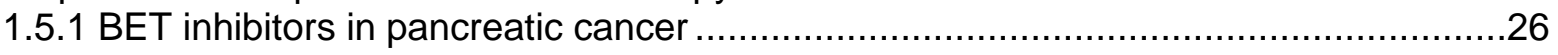

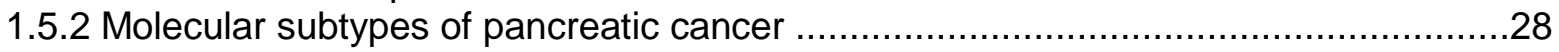

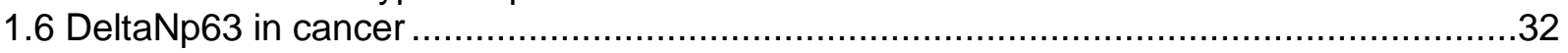

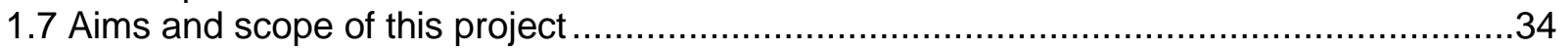

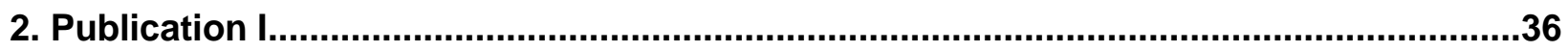

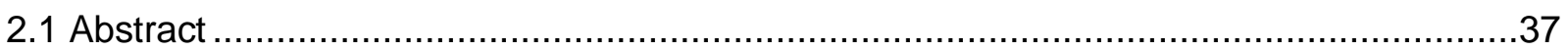

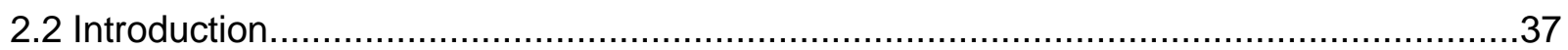

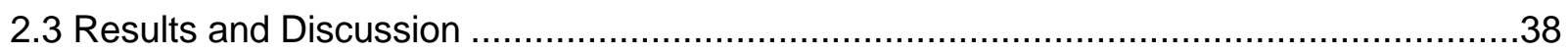

2.3.1 Super vs. Typical Enhancers: What is so bad about being typical? ..........................38

2.3.2 Picking the petals of ROSE: an enhancer is super, an enhancer is not super...........42

2.3.3 Super enhancer subcomponents: one for all or all for one? ................................4

2.3.4 Enhancer RNAs: whether bystanders or effectors, they are anyways a great help....45

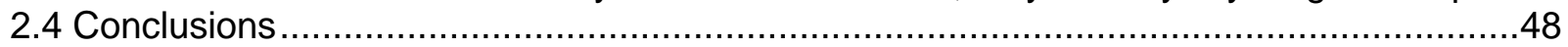

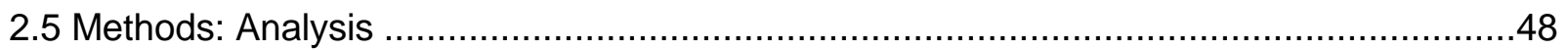

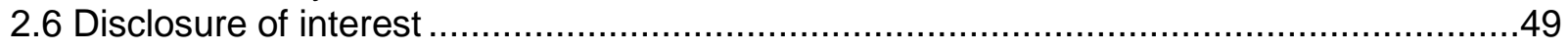

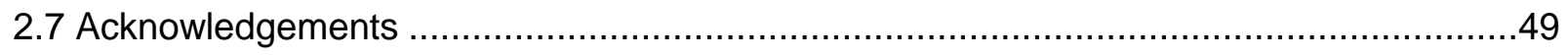

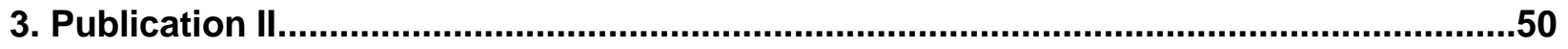

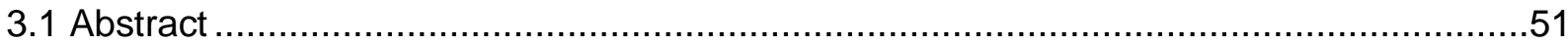

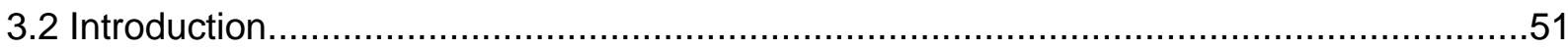

3.3 Bromodomain and Extraterminal Inhibitors in Pancreatic Cancer ................................53

3.3.1 Role and Effects of Bromodomain and Extraterminal Inhibitors in Pancreatic Cancer 
3.3.2 Bromodomain and Extraterminal Inhibition and Metastasis in Pancreatic Cancer......55

3.3.3 Effect of Bromodomain and Extraterminal Inhibition on Distal Regulatory Elements..57

3.4 Histone Deacetylase Inhibitors in Pancreatic Cancer..................................................59

3.4.1 Role and Effects of Histone Deacetylase Inhibitors in Pancreatic Cancer .................60

3.4.2 Histone Deacetylase Inhibition Role at Distal Regulatory Elements..........................61

3.5 Enhancers as an Optimal Paradigm for Therapeutic Targeting of Pancreatic Cancer ......64

3.6 Targeting Transcription Factors in Pancreatic Cancer: A Code for Specificity...................66

3.7 Conclusions: Unconventional Epigenetic Agents Should be Used in Unconventional Ways

3. Methods/Appendix A: Parameters for data analysis used in figure generation

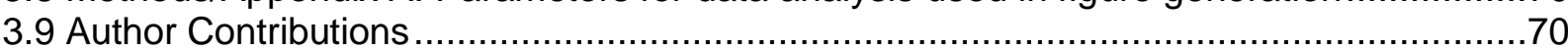

3.10 Acknowledgements ................................................................................

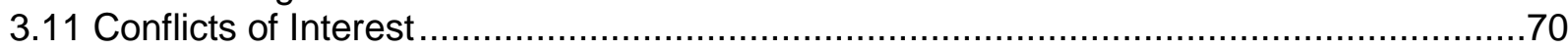

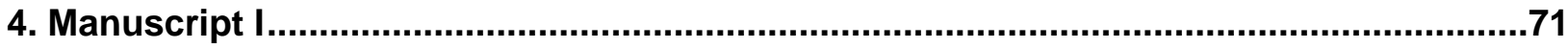

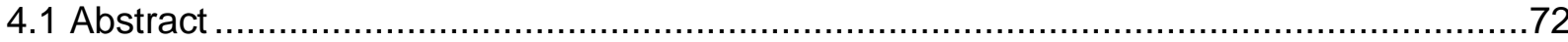

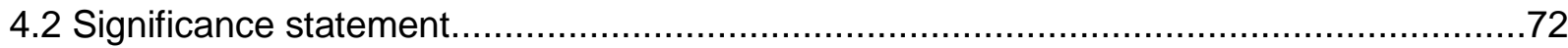

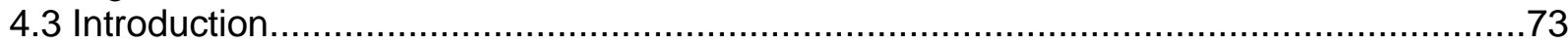

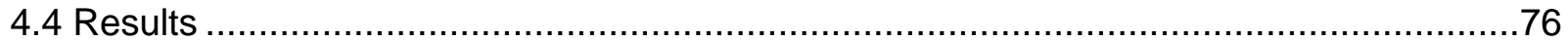

4.4.1 DeltaNp63 is a major driver of differential gene activation in specific pancreatic cancer cell lines and patient-derived xenografts ............................................................. 76

4.4.2 L3.6pl and BxPC-3 highly express deltaNp63 and are representative models for the squamous subtype of pancreatic cancer .............................................................. 79

4.4.3 Depletion of deltaNp63 alters the molecular identity of squamous pancreatic cancer

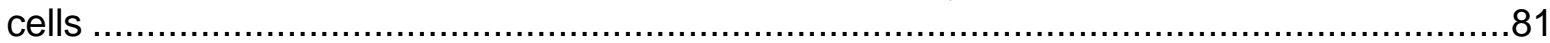

4.4.4 DeltaNp63 exerts its effects through activation of super enhancers .......................83

4.4.5 Super enhancers in the squamous subtype are highly dependent on deltaNp63.......86

4.4.6 DeltaNp63 cooperates with other transcription factors to activate target genes associated with worse prognosis......................................................................... 88

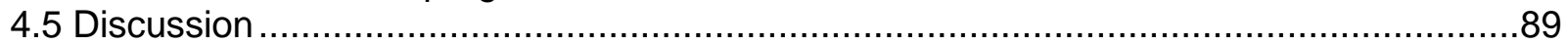

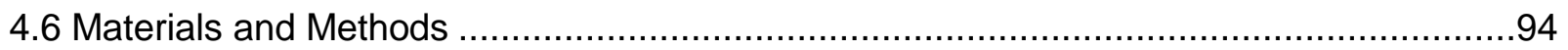

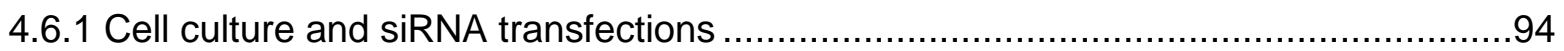

4.6.2 Proliferation and sphere formation assays.......................................................94

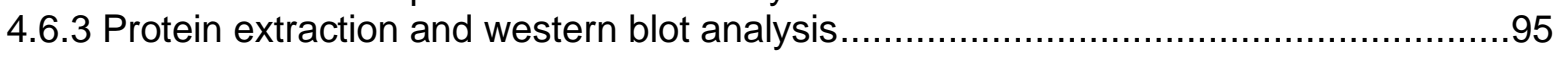

4.6.4 RNA isolation and quantitative real-time PCR (qPCR) …...................................95

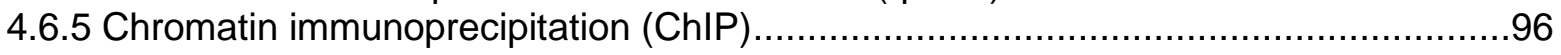

4.6.6 Assay for transposase-accessible chromatin (ATAC) ……..................................97

4.6.7 Library preparation for RNA, ChIP, and ATAC-seq and next-generation sequencing 97

4.6.8 Bioinformatic analysis for ChIP and ATAC-sequencing ........................................98

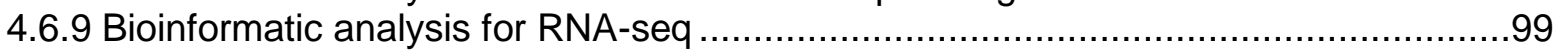

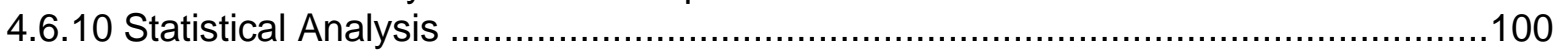

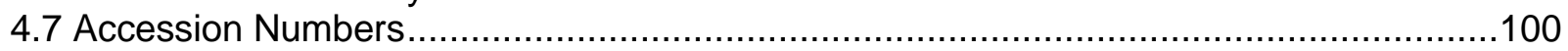

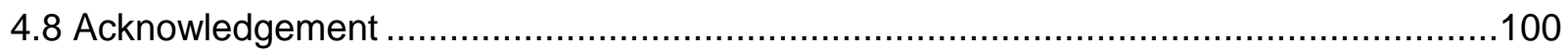

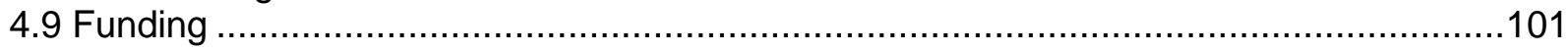

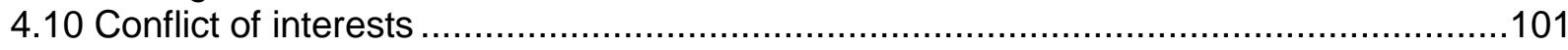

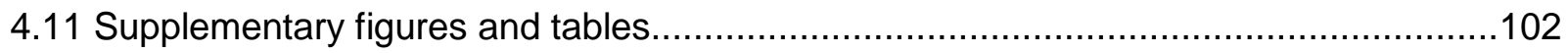

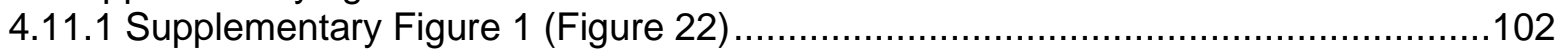

4.11.2 Supplementary Figure 2 (Figure 23) .......................................................103 


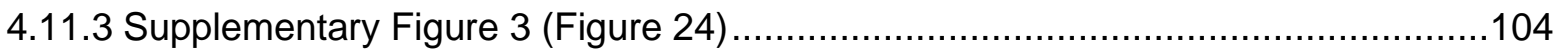

4.11.4 Supplementary Figure 4 (Figure 25) ………...........................................105

4.11.5 Supplementary Table S1 (Table 1) Sequences for primers used in this study (5'-3')

4.11.6 Supplementary Table S2 (Table 2) Accession numbers for next-generation datasets used in this study (ChIP and ATAC-seq) ...........................................................106 4.11.7 Supplementary Table S3 (Table 3) Accession numbers for next-generation datasets used in this study (RNA-seq).

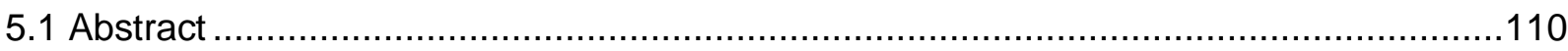

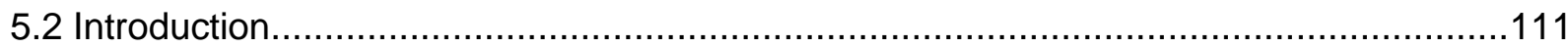

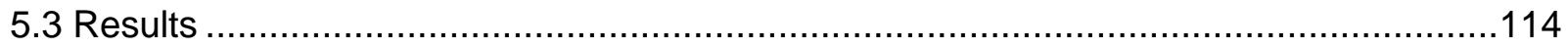

5.3.1 BET inhibitors sensitize normal and resistant pancreatic cancer cells to Paclitaxel .114

5.3.2 JQ1 reverses the regulation of transcriptionally-activated genes in resistant cells ...116

5.3.3 BRD4 is gained in putative enhancer regions of transcriptionally-activated genes in resistant cells

5.3.4 Gained super enhancers in resistant cells drive programs leading to poorer prognosis

5.3.5 BET-dependent genes respond differentially to low and high doses of JQ1 ..........122

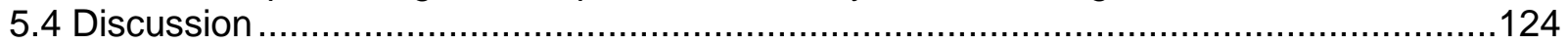

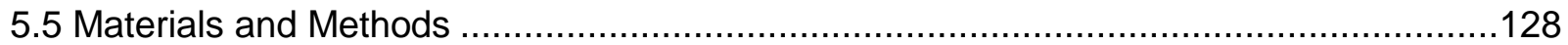

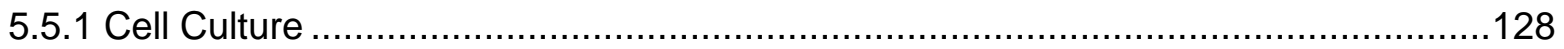

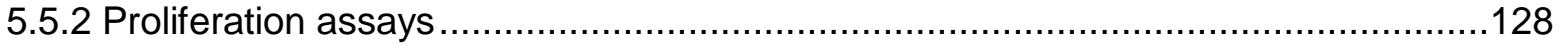

5.5.3 RNA isolation and cDNA synthesis .........................................................129

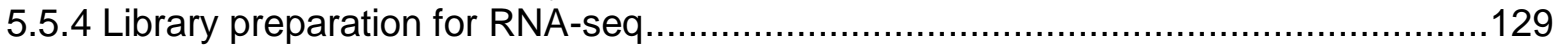

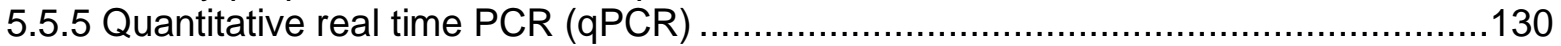

5.5.6 Chromatin immunoprecipitation (ChIP-seq) ……….....................................130

5.5.7 Chem- seq for biotinylated JQ1 …………….........................................131

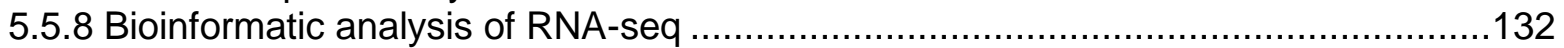

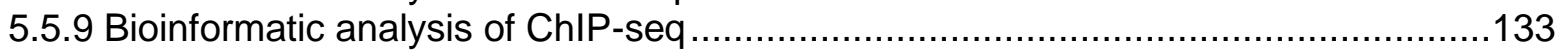

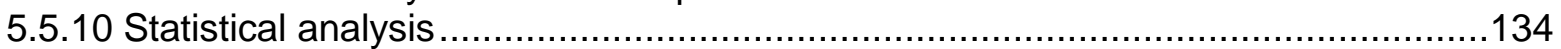

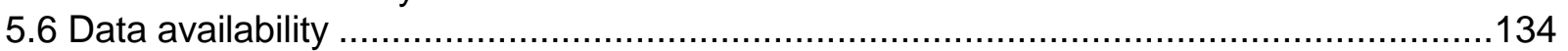

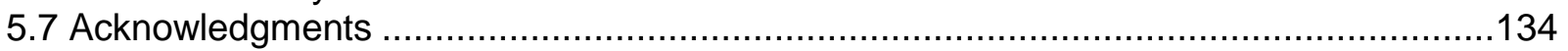

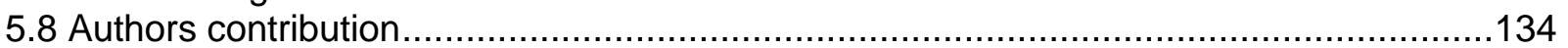

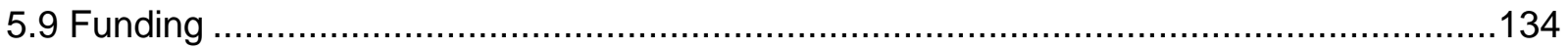

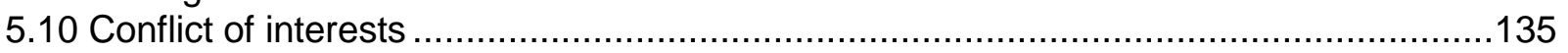

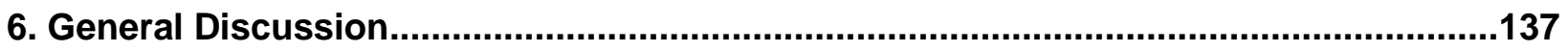

6.1 Enhancers as an emerging therapeutic target in malignancy ....................................137

6.2 Super enhancers as an important subset of enhancer elements...............................142

6.3 Transcription factors circuitry in context-specific gene regulation..............................145

6.4 DeltaNp63 as an activator of gene transcription.................................................147

6.5 Epigenetic approaches in precision-based medicine ..............................................149

4.6 BET inhibitors as eminent epigenetic modulators in cancer .....................................151

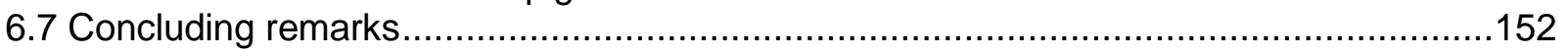




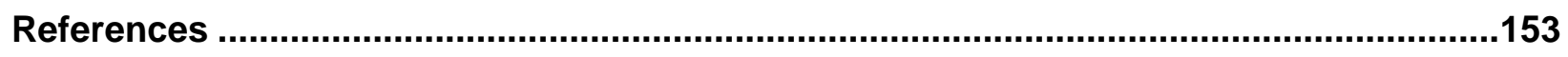

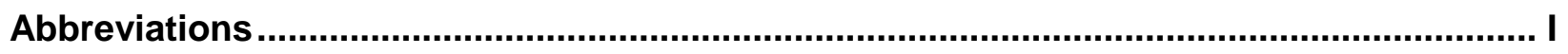

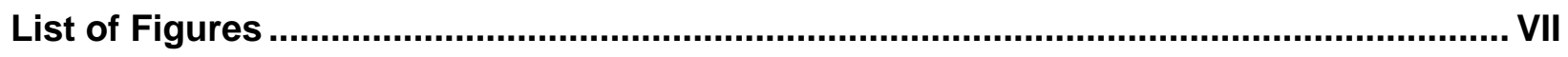

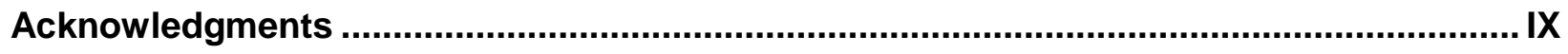

Curriculum Vitae ..................................................................................................... XI 


\section{Summary}

Enhancer elements comprise of regions of DNA that are distal to gene promoters with a characteristic capacity to affect and regulate gene transcription. Enhancers are enriched in a highly context-specific manner allowing for intricate control of gene expression. Current studies endeavor to elucidate the mechanisms underlying enhancer activation and function to ultimately exploit their specificity in targeted therapeutics. Due to the reported addiction of cancer to aberrant gene transcription, targeting enhancer elements is a promising therapeutic target in various malignancies. In this project, we conduct a series of studies with the general aim of extending the knowledge about the molecular mechanisms by which enhancers drive aberrant transcription in cancer. We focus on epigenetic modulation to exploit enhancer elements as therapeutic or prognostic targets.

In the first study included in our project, we evaluated the importance of the super enhancer subcategory of distal regulatory elements in a breast cancer cell line where estrogen plays an important role in driving gene expression through enhancers. Super enhancers are claimed to be a highly active subgroup of distal regulatory elements that is abundantly enriched with transcription factors, span long stretches of DNA, and exhibit preferential efficacy in driving major transcriptional programs in cancer. We identified super enhancers related to estrogen in this system using the standard algorithm and failed to observe a distinct high efficacy of super enhancers compared to typical enhancers. By varying the settings of this algorithm, we also uncovered biases in enhancer identification that extensively influence the results. On the other hand, we observed that major targets of estrogen activation showed a preference for association with super enhancers and concluded that they may indeed tend to regulate the transcription of master regulators. Accordingly, we concluded that the focused attention given to 
super enhancers should not lead to disregarding typical enhancers which also play a significant and important role in gene transcription regulation.

Consequently, in the second study we reviewed the role of enhancers in pancreatic cancer, a malignancy with exceptionally low survival rates. We focused on the application of epigenetic modulators, such as bromodomain and extraterminal proteins inhibitors and histone deactylase inhibitors, in targeting enhancer elements and speculated about mechanisms underlying the reported synergy between these two inhibitors. Interestingly, we used publicly available data to further study the context-specificity of enhancers. Notably, we observed a tendency where the same oncogenic target gene is activated by different enhancers in various systems due to differential expression of transcription factors.

Accordingly, we expanded our studies in pancreatic cancer and uncovered a group of subtypespecific super enhancers that drive the cells into a squamous phenotype which correlates with a particularly poor prognosis. Studying the general activation epigenetic profiles of different pancreatic cancer cell lines identified deltaNp63 as a major driver of the squamous molecular identity in cells and patient-derived xenografts. Moreover, extensive analysis of the role of deltaNp63 in driving a more aggressive phenotype uncovered the implication of super enhancers which are supported by a network of interconnected and differentially expressed transcription factors. This pattern resembles the reports of transcription factor regulatory circuitry driving the pluripotent molecular identity of embryonic stem cells. Identification of the same pattern governing differentiation into specific molecular subtypes in pancreatic cancer opens the door to precision-based medicine approaches targeting this circuitry in this particular subtype. 
Finally, we further investigated the role of enhancer elements in the context of chemotherapeutic resistance in pancreatic cancer. Interestingly, we observed that proinflammatory and migratory programs are activated in paclitaxel-resistant cells via activation of BET-dependent enhancers. Furthermore, we observed that BET inhibition sensitizes resistant and sensitive cells to paclitaxel. Notably, super enhancers that we observed to be enriched in resistant cells were associated with genes that correlate with poor prognosis. This study confirmed the patterns we uncovered in the other studies where enhancers and super enhancers drive aberrant transcription activation in cancer and present a promising target for patient treatment.

Altogether, this project resulted in 2 peer-reviewed publications in the journals of Transcription and Epigenomes, one manuscript that has been peer-reviewed and is currently under revision in Proceedings of the National Academy of Sciences of the United States of America (PNAS), and another manuscript in preparation for submission. These publications/manuscripts join the growing body of literature investigating the role of enhancers in malignancy and aim to guide new approaches for precision-based medicine. 


\section{CHAPTER 1}

\section{General Introduction}

This chapter provides a general overview extending the background information provided in each publication/manuscript and outlining the general aim of all the studies included in this project.

No published data is included in this chapter. 


\section{General Introduction}

\subsection{Epigenetic regulation of gene transcription}

Tightly regulated gene transcription is crucial for homeostasis and disease-free survival. Therefore, cells require systems to ensure the proper expression of genes in a spatial and temporal manner. Epigenetic regulation enables cells to control gene transcription via sequence-independent mechanisms including modification of histones, DNA methylation, nucleosome remodeling, and non-coding RNAs (ncRNAs) (Figure 1) [1]. DNA methylation, especially 5-methyl cytosine, was first reported in 1963 and shortly after was linked to repression of gene transcription [2-4]. DNA inside the nucleus is compacted by forming nucleosomal subunits including approximately 147 base pairs wrapped around a histone octamer composed of pairs of Histone 2A $(\mathrm{H} 2 \mathrm{~A})$, Histone 2B $(\mathrm{H} 2 \mathrm{~B})$, Histone $3(\mathrm{H} 3)$, and Histone $4(\mathrm{H} 4)$ [5]. This protein-DNA complex is known as chromatin. As many modifications of these histones have been identified, they were soon found to faithfully correspond to the states of gene transcriptional activation [6-9]. Additionally, the compaction of DNA via nucleosomes was observed to play a role in controlling gene transcription and the modulation of its structure by chromatin remodelers has been implicated in gene transcription regulation [10-13]. While the mechanism of action of ncRNAs is still not fully clear, they were reported to mediate epigenetic regulation of gene transcription via recruitment of epigenetic modulators to their target genes [14-17]. Altogether, these various tools work intricately and cooperatively to orchestrate gene expression regulation. The most broadly studied aspect of these mechanisms is posttranslational histone modifications as they create a versatile code which plays a significant role in gene transcription activation and repression. 


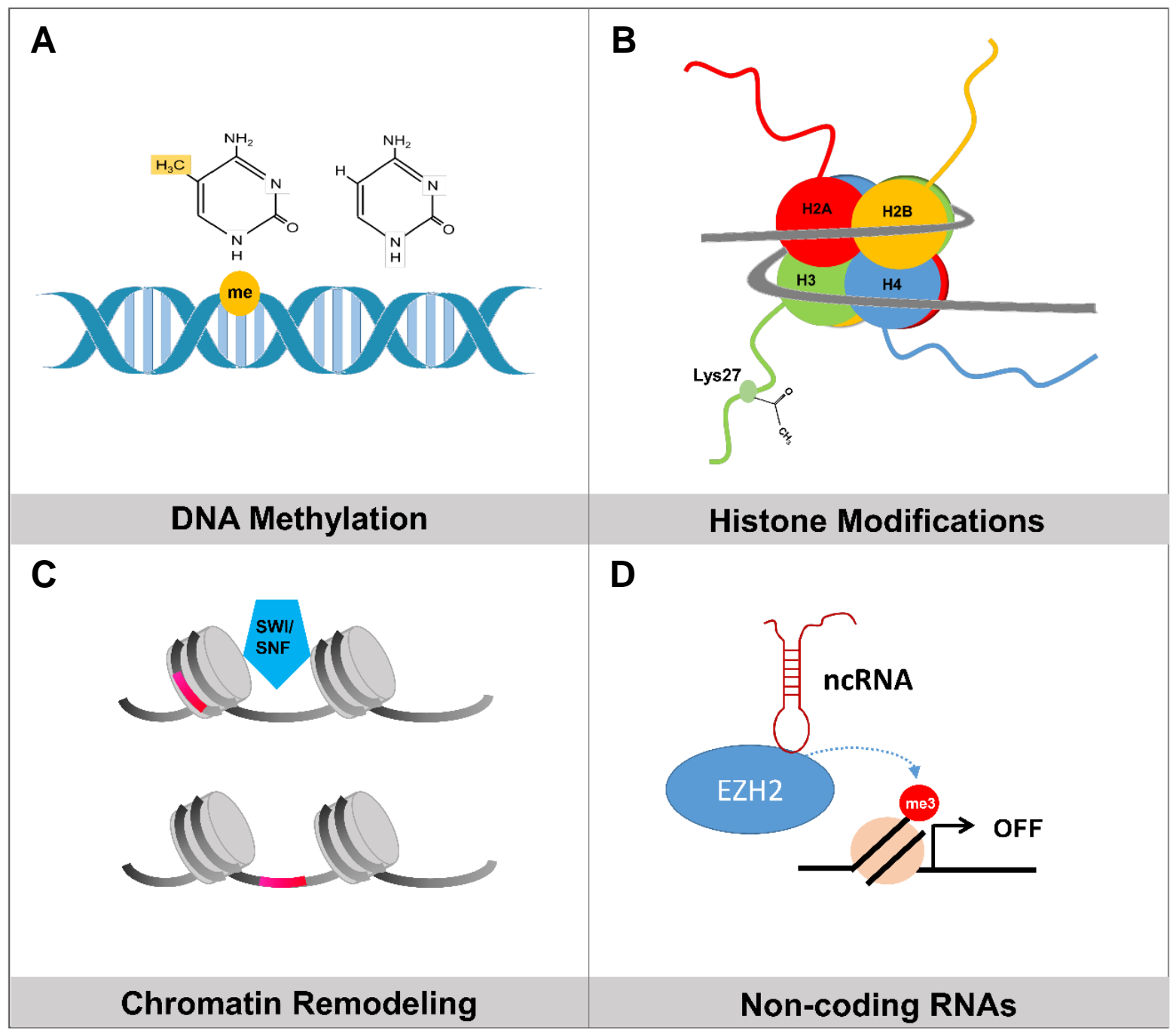

Figure 1: Mechanisms of Epigenetic Gene Regulation. Epigenetic regulation of gene transcription is mediated by various mechanism shown above as simplified diagrams including DNA methylation, histone modifications, chromatin remodeling, and non-coding RNAs. (A) Methylated cytosine is shown as part of the DNA helix as an example for DNA methylation. (B) A graphic representation for the nucleosome including an octamer of $\mathrm{H} 2 \mathrm{~A}, \mathrm{H} 2 \mathrm{~B}, \mathrm{H} 3$, and $\mathrm{H} 4$. An example of histone modifications is shown for Lysine 27 on the tail of $\mathrm{H} 3$ which is acetylated. (C) A chromatin remodeler such as the SWI/SNF complex changes the structure of the chromatin affecting the accessibility of a certain stretch of DNA. (D) Non-coding RNA recruiting an epigenetic modulator leading to the repression of gene transcription.

\subsection{The histone code}

\subsubsection{Active histone marks}

Numerous post-translational histone modifications are associated with active transcription. The most common of these modifications are acetylation of the lysine residues of $\mathrm{H} 3$ and $\mathrm{H} 4$ [18]. 
Histone acetylation was first reported in 1964 and was initially considered to affect transcription by neutralizing the positive charge of histones leading to a more relaxed and transcriptionpermissive chromatin structure [19-21]. However, as acetylation was found to have a minimal effect on chromatin structure [22], more complex mechanisms were proposed to explain the correlation of active transcription with histone acetylation. Protacio et al. [23] observed an increased rate of transcription for DNA upon acetylation of histones in a manner similar to total absence of the histone tails. Additionally, Wang et al. [24] showed that acetylation alters the alpha-helical content of histone tails. Most importantly, it was later uncovered that these marks create new binding motifs and form a "code" that can be recognized by transcription activators $[25,26]$. While many lysine residues are acetylated, the most known and studied is acetylation of Histone 3 Lysine 27 (H3K27ac) which shows the highest correlation to active gene transcription [27]. In addition, acetylation of Histone 4 Lysine 16 (H4K16ac) was found to be associated with decreased compaction of chromatin and acetylation of Histone 4 Lysine 5 and Lysine 8 (H4K5ac and H4K8ac) [28]. Furthermore, acetylation of Histone 3 Lysine 9, 14, 18, and 23 (H3K9ac, H3K14ac, H3K18ac, and H3K23ac) were found to correlate with active gene transcription [21].

Acetylation of lysine residues is not the only histone mark that is associated with gene activation. Methylation of lysine can also occur on the hydrogen of its primary amine which can be mono-, di-, or tri- methylated [29]. Mono-methylation and tri-methylation of Histone 3 Lysine 4 (H3K4me1 and H3K4me3) are known marks for gene activation [1, 30]. Furthermore, trimethylation of Histone 3 Lysine 36 is enriched at the 3'-end of transcriptionally active genes [31]. Mono-ubiquitination of Histone H2B Lysine 120 (H2Bub1) has also been found to correlate with highly expressive genes [32]. Altogether, these marks provide the opportunity to interpret gene activation profiles in different systems with high accuracy and precision (main active histone marks shown in Figure 2A). 


\subsubsection{Repressive histone marks}

Most common repressive histone modifications include methylation [33]. Histone methylation was first reported along with acetylation by Allfrey et al [19]. Methylation can occur either on histone lysine or arginine residues and is usually observed at the same histone lysine side chains that can be acetylated in the case of transcription activation [21, 33, 34]. Thus, acetylation and methylation are usually mutually exclusive at these residues and clearly indicate the state of transcription activation [35]. As previously mentioned, H3K27ac is a mark which highly correlates with the activation state while its counterpart, tri-methylation of Histone 3 Lysine 27 (H3K27me3), is equally predictive of gene inactivation [36, 37]. Furthermore, trimethylation of Histone 3 Lysine 64 (H3K64me3) was found at compacted chromatin regions known as heterochromatin [38]. Moreover, methylation of Histone 3 Lysine 9 is one of the most studied repressive histone marks of gene inactivation [39-41]. Acetylation can also act as a repressive mark as shown by acetylation of Histone 4 Lysine 20 (H4K20ac) which usually does not overlap with H3K27ac and is localized at genes that are minimally expressed [42]. Furthermore, mono-ubiquitination of Histone H2A Lysine 119 (H2Aub1) is a known repressive mark as the big ubiquitin moiety perturbs the dynamics of the nucleosomal architecture [43]. H2Aub1 was found to inhibit transcription elongation machinery and attenuate the recruitment of factors increasing the permissiveness of chromatin [44]. In order to render these histone marks functional (main repressive histone marks shown in Figure 2B), a group of tightly regulated enzymes should be involved to mediate the addition and elimination of these chemical moieties. Furthermore, a group of factors that can recognize these marks and mediate downstream effects is highly crucial for functional epigenetic regulation. These factors are called "writers", "erasers", and "readers", respectively. 

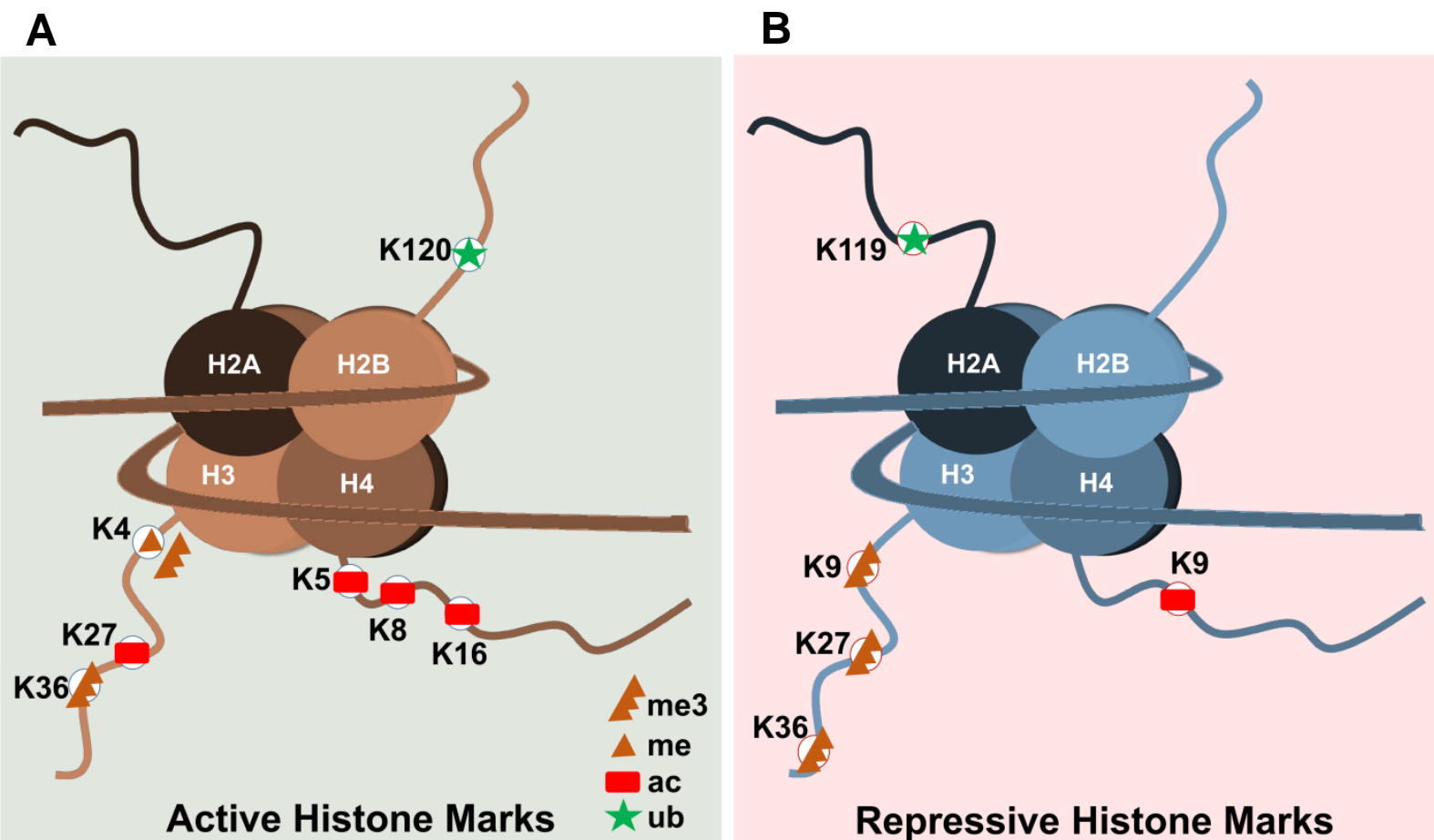

Repressive Histone Marks

Figure 2 : The main histone modifications acting as active and repressive marks. A representative diagram of a nucleosome showing the most studied histone tail modifications with the active histone marks shown in panel (A) and repressive marks shown in (B). me3: tri-methylation; me: methylation; ac: acetylation; ub: ubiquitination; K: Lysine.

\subsubsection{The epigenetic machinery regulating the histone code}

As histone modifications serve as a major regulator of gene transcription, meticulously controlled processes are involved in their maintenance. First of all, writers act by selectively adding these chemical moieties to a specific histone residue. Histone acetyltransferases (HATs), such as p300 and CREB-binding protein (CBP) , transfer an acetyl group from acetylCoA to histone tails [21]. Protein arginine methyltransferases (PRMTs) catalyze the methylation of arginine moieties on histones [45, 46]. Additionally, histone methyltransferases (HMTs) mediate the methylation of lysine moieties and include suppressor of variegation 3-9 homolog 1 (SUV39H1) which methylates Histone 3 Lysine 9 and mixed-lineage leukemia 3/4 (MLL3/MLL4) which methylate Histone 3 Lysine 4 [1, 47, 48]. Furthermore, the polycomb repressive complex 
1 (PRC1) and 2 (PRC2) are extensively studied complexes which mediate H2Bub1 and trimethylation of Histone 3 Lysine 27, respectively [49]. Additionally, ubiquitination of $\mathrm{H} 2 \mathrm{~B}$ is mediated by an E3-ligase which comprises two RING finger proteins, namely Ring Finger Protein 20/40 (RNF20/RNF40) [50].

The epigenetic machinery also includes enzymes that remove histone marks called erasers [51]. They include histone deactylases (HDACs) which mediate the removal of lysine acetylation and consist of multiple classes that can also mediate de-acetylation of non-histone proteins [52]. While methylation was previously considered to be an irreversible modification, lysine-specific histone demethylase 1A (LSD1) was identified in 2002 as a selective mediator of the demethylation of Histone 3 Lysine 4 [33, 53]. Another class of de-methylases has since been reported, namely Jumonji $\mathrm{C}$ domain-containing demethylases which can reverse mono-, di-, and tri-methylation [54].

Eminent factors in the epigenetic machinery include readers that recognize histone marks and recruit various effectors [55]. An extensively studied example is the Bromodomain and Extraterminal (BET) family which comprise of two bromodomains including a hydrophobic pocket that can interact with acetylated lysine and endows the BET family with the ability to read acetyl marks on chromatin [26]. Methylated lysine is recognized by the Royal superfamily members like Tudor- and chromo-domain proteins as well as PHD fingers [56, 57]. Altogether, epigenetic writers, erasers, and readers cooperatively mediate epigenetic regulation via histone modifications and serve as a lucrative target to manipulate gene expression in various systems (Figure 3). 


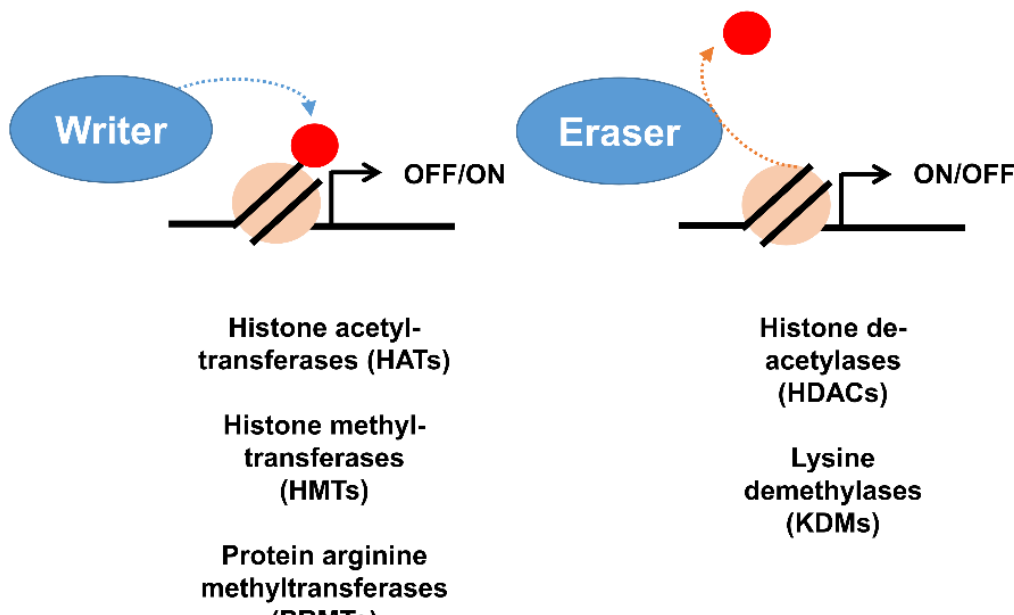

(PRMTs)

Figure 3 : Epigenetic writers, erasers, and readers. Factors forming the epigenetic machinery by which histone modifications regulate gene transcription. Writers add a chemical moiety to histone leading to the activation or inactivation of the gene. Erasers reverses this effect via removal of the histone mark and a reader recognizes the mark recruiting further effectors leading to the observed effects of histone modification. Examples of each class is mentioned below scheme.

\subsubsection{Histone marks signify distinct elements throughout the genome}

Histone marks do not act independently from each other but rather co-exist and co-operate to control gene transcription in what is known as "histone crosstalk" [58]. In addition to being an accurate predictor of gene activation status, many histone marks show preferences to certain elements in the genome. For example, H3K4me3 was reported to be highly enriched at gene promoters, stretches of DNA where gene transcription initiation originates [59,60]. On the other hand, H3K4me1 usually marks active distal regulatory elements (enhancers), which are stretches of DNA that can distally enhance gene transcription [60,61]. High levels of acetylation of $\mathrm{H} 3$ and $\mathrm{H} 4$ are usually observed in regions occupied by H3K4me3 [18, 62]. H3K27ac is enriched on active enhancers and promoters and usually overlaps with H3K4me1 and H3K4me3, respectively [60]. In case of an overlap between the active marks of H3K4me3 or H3K4me1 with the repressive histone mark H3K27me3, a promoter or enhancer is said to be in a bivalent state $[63,64]$. Bivalent genes have poised promoters or enhancers that are primed to 
be quickly activated if a temporal or spatial need for gene activation arises [65]. Understanding the trends and correlations of various histone marks has helped in the study of various elements in the genome. A graphical representation of histone marks on promoters and their states is depicted in Figure 4.

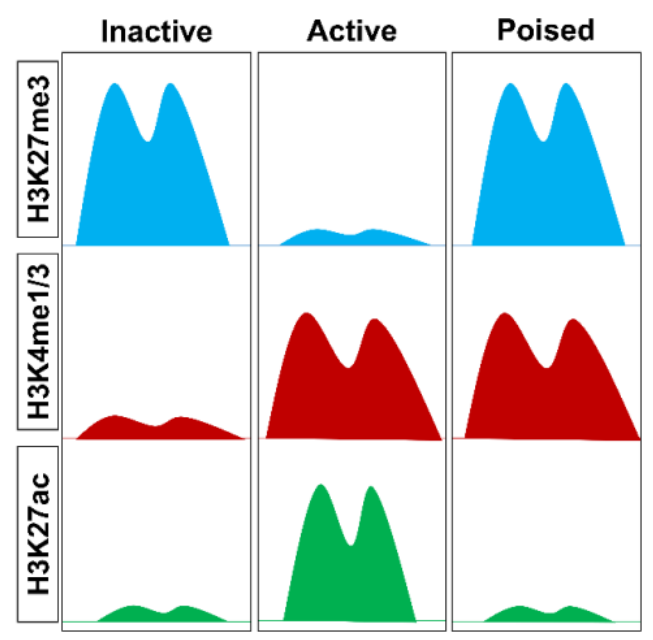

Figure 4 : Defining histone marks of active, inactive, and poised promoters and enhancers. A diagram depicting the distribution of histone marks that define the activation status of a promoter or enhancer. H3K27me3 is enriched at repressed elements while H3K27ac overlap with H3K4me1 on active enhancers and $\mathrm{H} 3 \mathrm{~K} 4 \mathrm{me} 3$ on active promoters. Poised elements are enriched for H3K27me3 along with $\mathrm{H} 3 \mathrm{~K} 4 \mathrm{me} 1 / 3$.

In addition to promoters and enhancers, insulators are important elements of the genome as they contribute to the $3 \mathrm{D}$ compartmentalization of chromatin which enables enhancers to interact with and affect their target genes $[66,67]$. These insulators are usually hyper-acetylated and are known to stop the expansion of $\mathrm{H} 3 \mathrm{~K} 27 \mathrm{me} 3$ into active regions $[68,69]$. Studying these elements via histone marks and chromatin accessibility in 5 different cell lines of different origins and characteristics has uncovered that only enhancers show a cell-specific pattern of enrichment compared to promoters and insulators which showed modest variability and high consistency among different cell lines [60]. 


\subsection{Enhancers and gene transcription}

\subsubsection{Gene transcription}

The assembly of the pre-initiation complex (PIC) at the promoter site marks the initiation of transcription and is followed by the phosphorylation of RNA polymerase II (RNA Pol II) at serine 5 of its C-terminal domain and the capping of nascent RNA [70, 71]. Within the first 100 nucleotides following the transcription start site (TSS), Pol II is temporarily paused by the negative elongation factor (NELF) and DRB-sensitivity inducing factor (DSIF) [70, 72, 73]. Thereby, promoter proximal pausing acts as a crucial rate-limiting step for gene transcription in metazoans [74]. To resume transcription, Pol II is phosphorylated at the serine 2 of its Cterminal domain by the positive elongation factor-b (P-TEFb) while NELF and DSIF are released upon phosphorylation $[75,76]$. This leads to the release of the promoter proximal pausing and transcription elongation proceeds until termination sequences are met and termination proteins lead to the cleavage of RNA followed by adenine capping [77].

An earlier rate limiting step in transcription regulation involves the assembly of the PIC at specific genes which is initiated by the binding of transcription factors (TFs) to specific genomic sequences [78]. PIC comprises mainly of TATA-Box Binding Protein (TBP), TFIIA, TFIIB, TFIIF, TFIIE, TFIIH, and RNA pol II [79]. These general transcription factors along with RNA Pol II interact with mediator which stabilizes the pre-initiation complex and is usually reported as one of its members $[80,81]$. Mediator is a very big complex comprising of multiple subunits and it plays a crucial role in the assembly and activation of PIC through forming a bridge between various sequence-specific TFs and the members of the PIC [78]. In addition to its important role at gene promoters, mediator is reported to connect initiating promoters with active distal enhancers through chromatin loop formation [82]. The first evidence of chromatin loop formation where a distal region can affect the transcription of a gene promoter was first reported in 1984 
by Dunn et al. [83] in bacteria. Approximately 20 years later, Cohesin, which was known to play a role in sister-chromatid adhesion, was revealed to orchestrate the formation of DNA loops with the help of the insulator, CCTC-Binding Factor (CTCF) and the cohesion loader, Nipped-B-Like (NIPBL) [84-86]. Mediator was found to bind Cohesin and NIPBL to bring active enhancers and promoters into near proximity [82]. As mediator is composed of approximately 30 subunits, it can have different conformations [87]. A conformation which includes the kinase module comprising of cyclin dependent kinase 8 (CDK8) and does not interact with RNA Pol II was shown to have more preference to active enhancers [88]. In general, regulation of gene transcription is not restricted to gene promoters but also extends to the distal regulatory elements like enhancers and insulators. A scheme showing the activation of gene transcription at promoters and enhancers is illustrated in Figure 5.

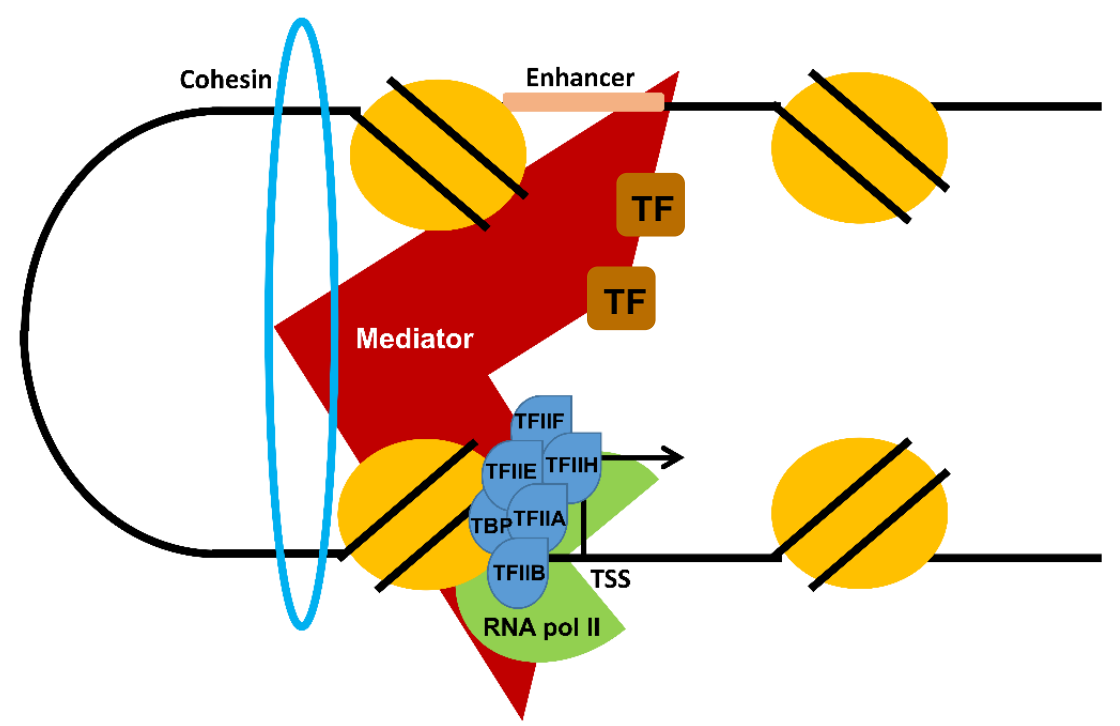

Figure 5 : A schematic diagram depicting the pre-initiation complex at the promoter site. Mediator is shown in the diagram linking enhancers and promoters, helping the DNA loop formation by binding to cohesion, stabilizing the pre-initiation complex, and acting as a bridge between transcription factors and PIC. 


\subsubsection{Distal regulatory elements (Enhancers)}

Enhancers are small genetic elements which are highly enriched in DNA sequences that can be bound by TFs and can augment the transcription of their target genes in a distal and autonomous manner [89]. The first enhancer element was reported in Xenopus oocytes and activated the H2A gene while localized at a distal upstream region [90]. This was followed by the identification of enhancer distal regulatory elements in different systems [91-93]. Soon after their discovery, enhancers were reported to drive differential transcriptional regulation in a more diverse and versatile manner than transcriptional regulation at promoters [94]. As enhancers are activated via binding of sequence-specific TFs and are not restricted to a certain promoter region, they provide a flexible platform for gene activation [95]. TFs use their adaptor characteristics of scanning and binding to specific regulatory sequences throughout the genome to activate transcription via recruitment of chromatin remodelers in addition to various activators $[96,97]$. Certain TFs are reported to be lineage-specific and drive the differentiation of certain states in the cell through activation of different constellations of enhancers [98, 99]. Moreover, it was reported that certain TFs have a preference to enhancers over promoters and vice versa [100]. Additionally, the pattern by which those TFs bind to their target sequence, whether towards the middle or the periphery correlates with certain patterns of recruitment for effectors and co-activators [101]. Furthermore, a subgroup of TFs called pioneer transcription factors have the ability to bind DNA in its compacted state, adding a level of regulation that is independent of histone marks and chromatin remodelers [102]. Examples of pioneer TFs include Forkhead Box A1 (FOXA1) and GATA Binding Protein 4 (GATA4) [97]. In conclusion, it is the combination of differentially expressed TFs and variously activated enhancers that enables the cell to tightly regulate gene expression (Figure 6). 


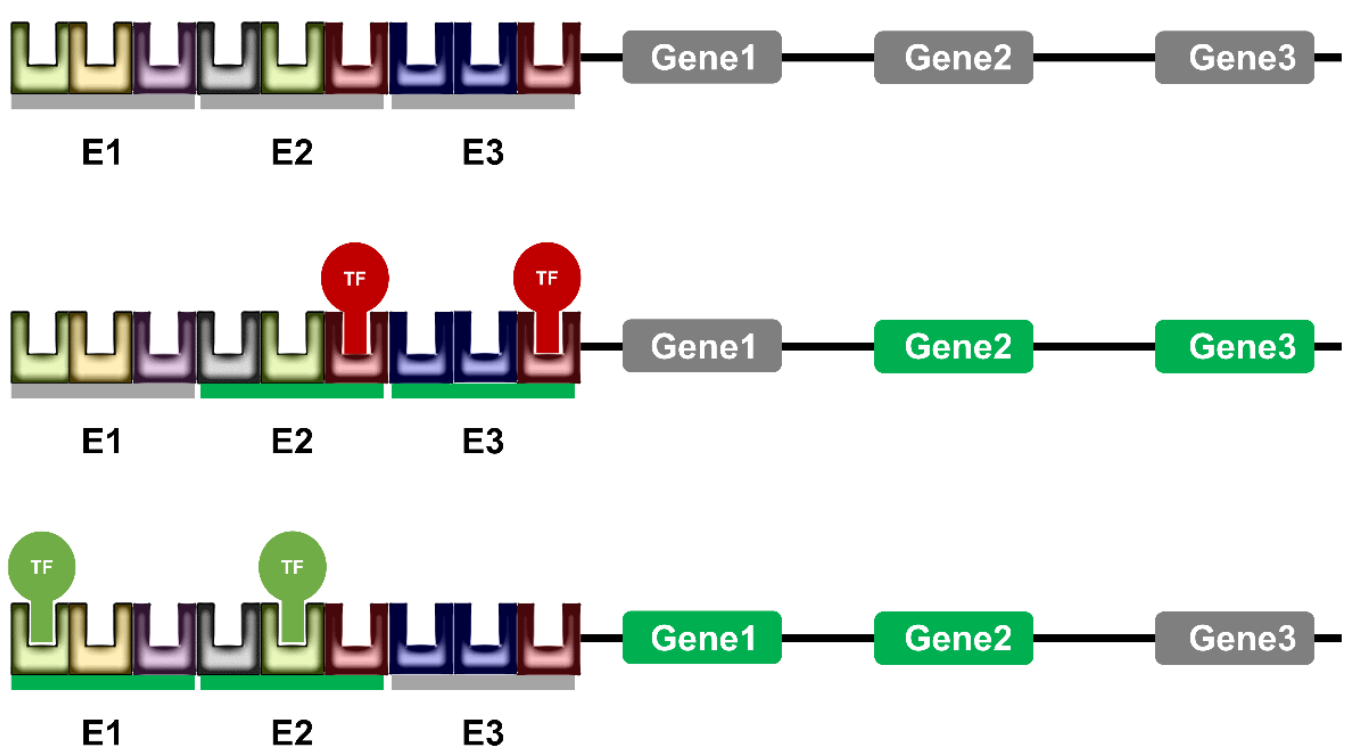

Figure 6 : Transcription factors and enhancers cooperate in differential gene activation. A simplified diagram showing the ability of different transcription factors to activate various sets of enhancers leading to the activation of different combinations genes.

Although enhancer sequences do not contain genes, they were found to be transcribed in contradiction to the general trends of energy conservation inside the cell [103]. The functions and mechanisms of the resulting products, named enhancer ribonucleic acids (eRNAs), are still not fully elucidated [104] . In general, eRNAs were reported to augment gene transcription as their knockdown led to decreased target gene transcription [105, 106]. Additionally, eRNAs were found to interact with NELF and facilitate promoter proximal pausing release [104]. Furthermore, chromatin loop formation and eRNAs production were reported to be preceding events before the activation of gene transcription [107]. Interestingly, the known tumor suppressor, tumor protein P53 (p53), was reported to exert its effects on certain enhancers that are not bound by it via p53-dependent eRNAs [108]. Studying the mechanisms of eRNA functions is expanding as techniques that can detect these highly unstable RNAs make use of labeling newly synthesized products to detect nascent RNA. These techniques include global run-on sequencing (GROseq) [109], transient transcriptome sequencing (TT-seq) [110], precision nuclear run-on sequencing (PRO-seq) [111], and chromatin run-on and sequencing (ChRO-seq) [112]. 
A crucial hurdle facing the investigation of the role of enhancers in transcription activation is the complexity of defining the target genes of each enhancer. In Figure 6, each target gene is given the same number as its enhancer for simplification purposes. In the cell, targets of enhancers are not necessarily in near proximity and can be separated by many unaffected genes [113]. Interactions between enhancers and their target genes are variable in different systems and show more tissue-specificity than differential activation of enhancers themselves [114]. Chromatin conformation capture assays to detect interactions between cis-regulatory elements were first established in 2002 and have been followed by many techniques that extended our knowledge about the interactions between enhancers and their target promoters [115-118]. Most importantly, these techniques identified specific compartments in the genome called topologically associating domains (TADs) that cultivate interactions inside them and isolate their constituents from the other compartments, thereby regulating the interactions between distal regulatory elements and their targets [119]. These TADs are flanked by insulators like CTCF and are highly conserved through different cells and comprise of regions that are characterized by a high rate of interaction [120-122].

Given their tissue- and system- specificity, enhancers have garnered attention as major players in gene transcription activation and drivers of certain programs and differentiation states. This led to the identification of further subgroups of enhancers such as shadow enhancers and super enhancers.

\subsubsection{Super enhancers}

Super enhancers (SEs) were first identified as major drivers of gene expression that are highly enriched by transcription factors binding sites and include clusters of highly active distal regulatory elements $[123,124]$. Target genes that are controlled by more than one enhancer were reported to exhibit higher tissue-specificity [114]. In accordance with this, super enhancers 
were observed to drive lineage-specific programs in various systems such as epithelial differentiation, mesenchymal pluripotency, and estrogen-dependent mammary gland malignancy [125-127]. Interestingly, SEs were not only observed in mammalian systems such as murine and human cells, but were also enriched in Zebrafish where approximately half of the observed SEs were proximal to their counterparts in mammals [128]. Super enhancers were reported to form a phase that is liquid-condensate-like where high rates of transcription occur [129]. These reports all confirm the important, though not fully elucidated role, of super enhancers in gene transcription regulation.

The Ranking of Super Enhancer (ROSE) algorithm is usually used to identify this subcategory of enhancers. The limitations and different settings of this algorithm are thoroughly discussed in chapter 2. Briefly, H3K27ac regions are stitched if the distances between them are less than the default 12.5 kilo base (kb). Afterwards, a density coverage file for a highly enhancer-enriched factor like mediator (MED1) or the BET family member, Bromodomain Containing 4 (BRD4), is used to calculate the density on these stitched regions. Enhancers with high density of these factors are deemed super enhancers (The ROSE algorithm approach is summarized in Figure 7A). Limitations of the algorithm include the stitching distance which is set on an arbitrary number that can be changed and lead to extreme changes of results (Figure 7B). Moreover, TSS inclusion is not accurate in the case of two H3K27ac peaks flanking a TSS leading to the inclusion of promoters in the midst of enhancers (Figure 7C). 
A
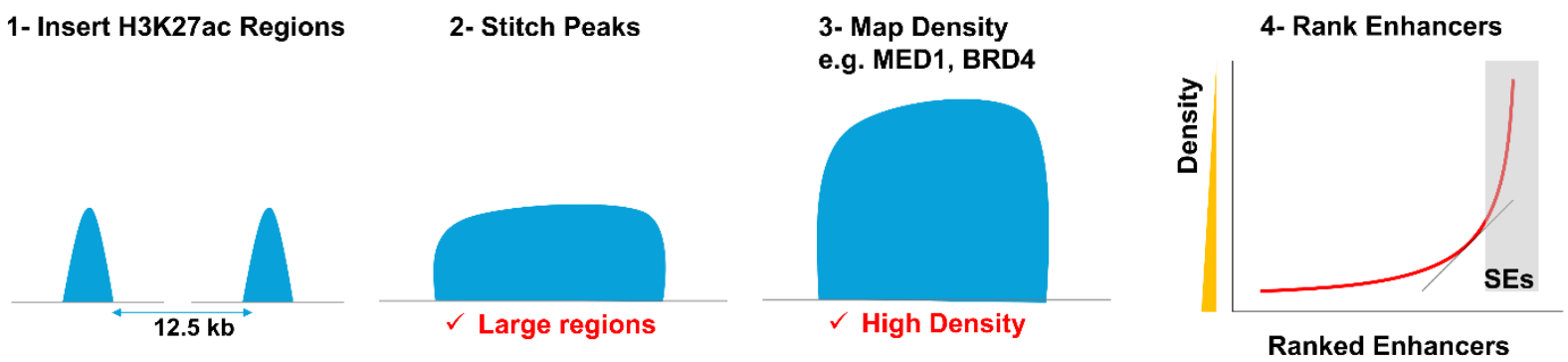

B

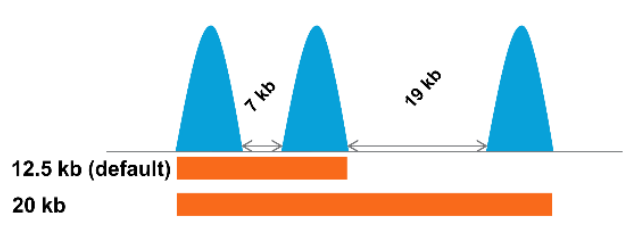

C

Figure 7 : ROSE Algorithm in defining super enhnacers. (A) Regions of H3K27ac are fed into the algorithm and regions that are less than $12.5 \mathrm{~kb}$ apart are stitched and the density of highly active factor on enhancers such as MED1 and BRD4 is used to rank enhancers with the ones having a higher density identified as super enhancers. (B) A diagram depicting the effects of changing the arbitrary cut-off point leading to identification of different enhancers. (C) The inclusion of TSS in the ROSE algorithm where even if regions around TSS are ignored, enhnacers including TSS are counted. This happens in case a TSS is present between two stitched peaks or if two TSS regions are included in one peak.

To solve the arbitrary selection of a stitching distance, a new algorithm was developed called Clustering of genomic REgions Analysis Method (CREAM) [130]. This algorithm uses a machine learning approach to define the stitching region for each constellation of enhancers as it stitches all possible peaks in near proximity and calculates the stitching region from the point where the difference to the maximum window size included in the combination is equal to zero. This ensures the implementation of variable stitching regions for each combination depending on the various window sizes flanked by it. CREAM identifies clusters of cis-Regulatory Elements (COREs) which overlap to a good degree with the identified SEs using ROSE. As SEs were found to associate with various diseases and anomalies, better algorithms to define those enhancers will be highly beneficial. 


\subsubsection{Enhancers and super enhancers in disease}

As enhancers and super enhancers play a crucial role in gene transcription activation, deregulation of these transcriptional hubs were naturally linked to imbalances and diseases. In fact, many single nucleotide polymorphisms (SNPs) linked to various diseases, such as cancer, Rheumatoid arthritis, Diabetes Mellitus type 2, and Alzheimer's disease, were reported to occur in super enhancer regions [131-133]. Interestingly, a clear preference of SNPs to enhancers compared to promoters and other elements was observed in Crohn's disease, systemic lupus erythymatosus, and breast and prostate cancer [134]. The eminent implication of enhancers in numerous diseases led to the development of the term "enhanceropathies" [135].

A chromosomal rearrangement in acute myeloid leukemia (AML) was found to bring an enhancer in the near proximity to the oncogenic MDS1 and EVI1 complex locus (MECOM), precipitating the malignancy [136]. Another example includes the implication of enhancers in therapy resistance in leukemia [137]. Additionally, DNA methylation at super enhancers was reported to be aberrantly changed in malignancies [138]. Amplification of enhancers has also been found to play a role in the pathophysiology of prostate cancer and neuroblastoma [139, 140]. Moreover, reprogramming of the enhancer landscape in pancreatic cancer was reported to play a significant role in promoting metastasis [141]. Due to the fact that cancer is a disease of aberrant transcription, the study of enhancer biology has become a focal point of study when investigating various malignancies [142]. Interestingly, few malignancies where a major driver mediates aberrant transcription are well-defined and provide a chance to study the mechanisms of enhancer implication and identify patterns that can be applicable in other types of cancers. The most known of these systems include hormonal regulation such as androgen receptors $(A R)$ in prostate cancer and estrogen receptors (ER) in ER-positive breast cancers. 


\subsubsection{Enhancers in ER-positive breast cancer}

Breast cancer is the most common diagnosed malignancy and one of the most eminent causes of cancer-related mortality in women around the world $[143,144]$. Therapeutic management of breast cancer has been significantly optimized by the identification of subgroups that respond differently to therapies based on their hormonal status [145]. Estrogen receptor positive $(E R+)$ breast cancer subtype represents $70 \%$ of breast cancer patients and is highly responsive to endocrine therapy [146]. ERa is a master transcription factor in breast cancer which can be activated by estradiol. This leads to conformational changes in the receptor, mediating its dimerization and subsequent binding to specific targets in the genome called estrogen response elements (EREs) [147]. ERa can also exert some effects that are independent of its gene expression effects and include activation of protein-kinase cascades and signaling pathways mediated through membrane-associated receptors [148].

The first characteristic observed when investigating estrogen localization throughout the genome was that it binds minimally to promoters and show an extreme preference to enhancer regions [149]. Silencing of estrogen-bound enhancers revealed different levels of enhancer hierarchy where estrogen binding sites are crucial for gene activation (predominant) or merely augmenting activation (supportive) [150]. Additionally, ER were linked to super enhancers activated upon ER activation as these generally included highly specific EREs [126]. It was also implied that ERa can bind to "canonical" EREs even in the absence of activation and is usually acting along with MED1 and BRD4 to activate enhancers [126, 151]. Indeed, ER binding was shown to recruit BRD4 to enhancer regions which enhanced eRNA production [152]. As estrogen positive systems largely mediate their effects via enhancers and the binding of specific domains, they provide an easily-defined system to study the role of enhancers. This knowledge can be used to test if the patterns discerned in this system apply to other less-defined systems. 
In the remainder of this chapter, focus will be directed at a challenging system where molecular mechanisms and subtypes are still under investigation.

\subsection{Pancreatic Cancer}

\subsubsection{Pathophysiology of pancreatic cancer}

Pancreatic cancer is well-known for its association with dismal survival rates and current studies are investigating various methods to optimize its therapy [153]. Recent findings implied that pancreatic cancer is highly related to enhancers [154]. Accordingly, studying the enhancer biology in pancreatic cancer is of great interest and benefit to patients. The pancreas has dual functions in the body where it regulates the levels of blood sugar on the endocrine level and mediates digestion via excretion of digestive enzymes on the exocrine level [155]. Interestingly, pancreatic cancer occurs with much higher incidence in the exocrinic pancreas, representing about $90 \%$ of pancreatic cancer patients in what is known as pancreatic ductal adenocarcinoma (PDAC) [156]. PDAC is most commonly preceded by the development of histologically distinct atypical legions called pancreatic intraepithelial neoplasms (PanINs) [157, 158]. A progression

model for pancreatic cancer has been proposed where a series of sequential genomic mutations in KRAS proto-oncogene (KRAS), cyclin dependent kinase inhibitor 2A (CDKN2A) and p53 lead to the progression from normal pancreata to PanINs to PDAC [159]. KRAS mutations lead to the constitutive activation of KRAS signaling and its downstream pathways leading to increased proliferation and altered differentiation and migratory potential [160, 161]. Both CDKN2A and p53 are tumor suppressors which in the case of mutation lead to unchecked growth and disabled protective responses to anomalies [162-164]. 
Epigenetic pathways also play a significant part in the initiation and progression of pancreatic cancer. For example CDKN2A can be hindered either by a genomic mutation or hypermethylation of its promoter sequence [165]. Furthermore, it was reported that krüppel like factor 4 (KLF4) acts as pioneer transcription factor that re-programs the gene transcriptional profile in the pancreas and leads to the development of PanIN legions in mice $[166,167]$. SWI/SNF related, matrix associated, actin dependent regulator of chromatin, subfamily A, member 4 (SMARCA4) is a subunit of the SWI/SNF chromatin remodeler complex and its deletion, similar to other known gene mutations, lead to the development of PDAC [168]. Altogether, aberrant epigenetic pathways are major players in PDAC and its implications are thoroughly discussed in chapter 3. A general scheme of the progression model with main genetic and epigenetic precipitants is depicted in Figure 8 (based on model suggested by Hruban et al. [159]).

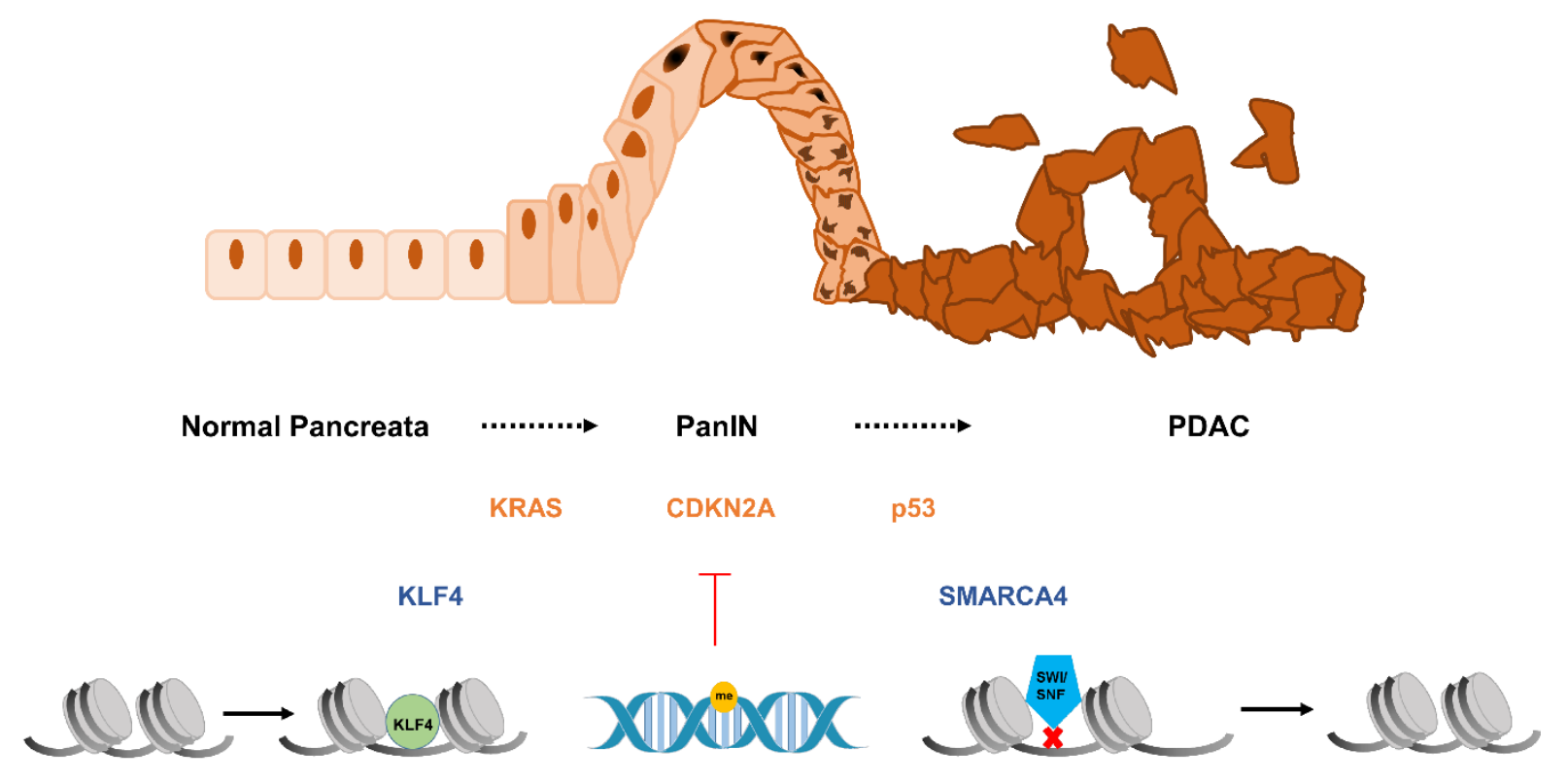

Figure 8 : Progression model for pancreatic cancer. Schematic representation of pancreatic cancer development from normal pancreata to PanINs to PDAC which is accompanied by mutations of KRAS, CDKN2A, and p53. Epigenetic factors playing a role in pancreatic cancer progression asre also depicted including KLF4 acting as a pioneer transcription factor, and SMARCA4 deletion leading to PDAC formation. 


\subsubsection{Therapeutic management of pancreatic cancer}

Incidence and mortality rates for pancreatic cancer are almost equal due to the low survival rates of this aggressive malignancy $[153,169]$. This is due to the fact that most patients present symptoms when the cancer is at later stages and has already metastasized to other regions [170]. Risk factors include familial history, smoking, excessive alcohol consumption and obesity [171]. Management strategies of pancreatic cancer include surgical resection for eligible patients and chemotherapy [172]. First-line chemotherapeutic options in pancreatic cancer patients include gemcitabine, Fluorouracil, nab-paclitaxel, or combinations including these agents [173].

Fluorouracil used to be the standard of care for pancreatic cancer patients in spite of its low efficacy [174]. Fluorouracil exerts its effects by inhibiting thymidylate synthase in addition to the interference with functions of DNA and RNA where its metabolites get incorporated in [175]. Fluorouracil has been brought back to first-line therapy as combination with other agents proved to increased its beneficial effects [176]. Gemcitabine affects replication and DNA synthesis by the virtue of being a nucleoside analog which gets incorporated into DNA hindering its synthesis. It also inhibits ribonucleotide reductase, the enzyme mediating the synthesis of deoxyribonucleotides [177]. Gemcitabine is considered one of the first-line available chemotherapeutic agents affecting, though modestly, the survival-rate of patients and improving symptoms [178]. Interestingly, combination of gemcitabine with other agents has proved to be more beneficial to patients [179]. A particularly interesting combination for gemcitabine is with nab-paclitaxel which showed higher efficacy in increasing survival of patients [180, 181]. Paclitaxel stabilizes microtubules forming the mitotic spindle and prevents their depolymerization, thereby pushing the cells into an arrested cell cycle state and apoptosis [182]. Notably, most pancreatic cancer patients show low responsiveness rates to chemotherapeutic 
agents whether alone or in combination due to resistance [183]. This forms one of the big hurdles in the management of pancreatic cancer and current research is trying to promptly address the problem.

Resistance to chemotherapy can be inherent to the tumor or develop upon treatment [184]. Pancreatic cancer resistance to chemotherapy can stem from extrinsic mechanisms such as undetected micro-metastases and microenvironment-related conditions like hypoxia [185]. It can also be due to intrinsic mechanisms where genetic mutations and epigenetic pathways play a role in evading the toxic effects of chemotherapy. In spite of its positive effects on survival rates, most pancreatic cancer patients acquire resistance to gemcitabine [186]. One of the mechanisms associated with gemcitabine is the stabilization of mutant p53 which helps the cell to evade apoptosis [187]. Another mechanism is the deregulation of enzymes that metabolize gemcitabine [188]. Interestingly, paclitaxel was shown to decrease the levels of the gemcitabine de-activating enzyme, cytidine deaminase, thereby potentiating its effects [189]. Moreover, resistance to gemcitabine did not show cross-reactivity and resistant cells retain their sensitivity to paclitaxel [190]. On the other hand, resistance to paclitaxel itself can also occur due to increased expulsion from the cell by multidrug transporter P-glycoprotein, change in metabolism, and modulation of the target microtubule [184]. In conclusion, the current chemotherapeutic agents used in pancreatic cancer minimally but significantly affect the overall survival of patients and this effect is further attenuated by resistance. Accordingly, investigation of unconventional therapies is currently underway in order to optimize management and find alternative more effective therapies for pancreatic cancer.

\subsection{Optimization of pancreatic cancer therapy}

Many therapeutic targets have recently been identified in pancreatic cancer leading to the initiation of many clinical phase trials to validate the efficacy of their targeting. These therapies 
include anti-inflammatory drugs inhibiting interleukin-1 [191], inhibitors targeting the usually overexpressed epidermal growth factor receptor (EGFR) signaling [192], and targeting modulated DNA damage response by the use of poly-ADP ribose polymerase (PARP) inhibitors [193]. Inhibitors of epigenetic targets are also extensively studied and include HDAC and BET inhibitors [194-196]. Applications of these two classes of epigenetic inhibitors especially in the scope of enhancers are extensively discussed in Chapter 3 . In the following section, the mechanism of action and role of BET inhibitors in pancreatic cancer is slightly extended.

\subsubsection{BET inhibitors in pancreatic cancer}

Recent findings showed that inhibition of BET family proteins is effective, both alone and in combination with conventional chemotherapy, in decreasing pancreatic tumor growth in patientderived xenografts $[197,198]$. The BET family consists of BRD2, BRD3, BRD4, and the testisspecific BRDT [123]. All BET family members contain two conserved bromodomains, which enable them to recognize acetylation marks on the chromatin, and an extraterminal domain that interacts with other proteins [26, 199]. The bromodomain comprises of a hydrophobic pocket that can interact with acetylated lysines and enables the BET family to read acetyl marks on chromatin [26]. It was previously mentioned that P-TEFb is recruited to regions of promoter proximal pausing leading to its release and activation of transcription elongation. BRD4, the most studied of the BET family, is reported to recruit P-TEFb upon recognition of chromatin acetylation which leads to the phosphorylation of the Serine 2 of the RNA Pol II tails [26, 200, 201]. The Serine 2 phosphorylated RNA Pol II interacts with the WAC complex (WW domaincontaining adaptor with coiled-coil) which then recruits RNF40 and RNF20 complex [202]. This E3 ubiquitin ligase complex is responsible for the monoubiquitination of histone H2B lysine 120 tail which further promotes active transcription [203, 204]. (Figure 9) 

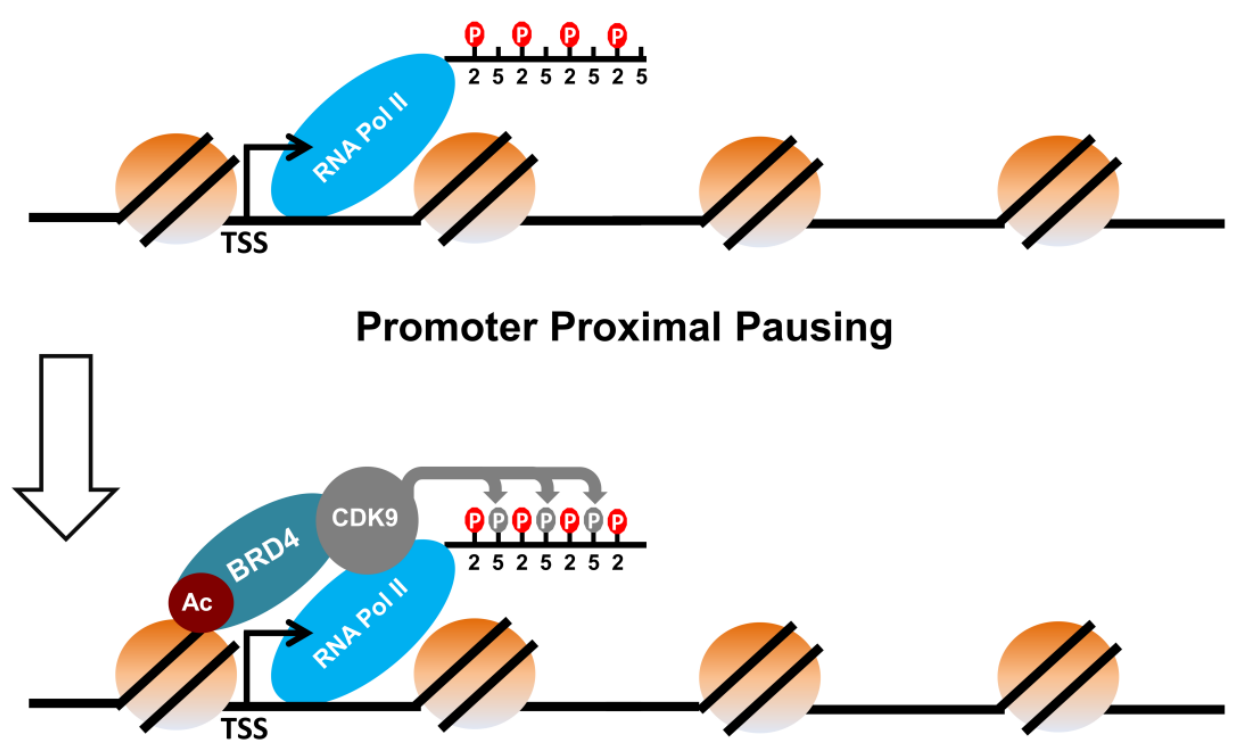

Promoter Proximal Release

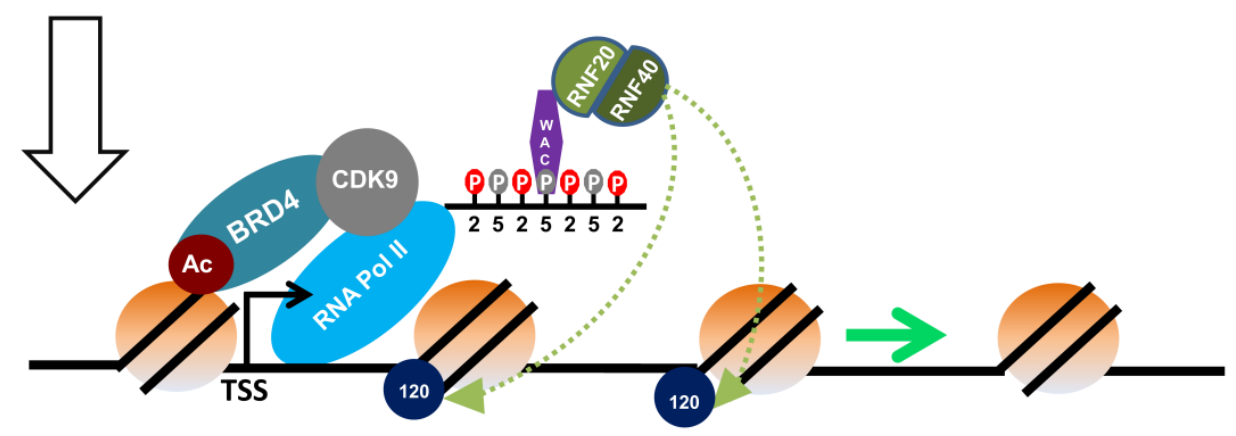

\section{Transcription Elongation}

Figure 9 : The role of BRD4 in transcription elongation. Acetylated histone marks are recognized by BRD4 which recruits CDK9 that phosphorylates RNA polymerase II C- terminal residues at the Ser2 position leading to promoter clearance. RNA Pol II Ser2-P interacts with WAC complex which recruits RNF20/40 complex. The E3 ligase RNF20/40 complex ubiquitinates H2B at lysine 120 (H2Bub1) and promotes transcription.

JQ1 is a thienodiazepine that displaces BRD4 from acetylated lysines by forming hydrogen bonds with a conserved asparagine residue that is situated in the hydrophobic pocket of BRD4 [205] (Figure 10). Many other BET inhibitors have also been developed, with some new agents having different specificity ratios toward BD1 or BD2 like I-BET151, I-BET762, and OTX-015 [205-207]. 


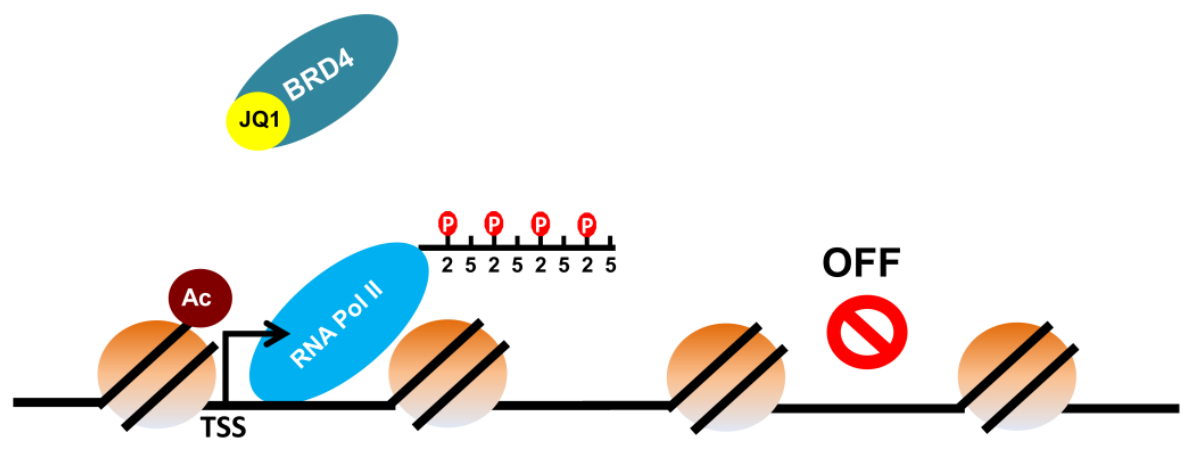

Figure 10 : The small molecule Bromodomain inhibitor JQ1. JQ1 competitively binds to BRD4 and inhibits the binding of BRD4 to acetyl lysine moieties on histone. This leads to the decrease of transcription of specific genes.

BET inhibitors have shown promising effects in sensitizing cells to other conventional therapies and are currently under investigation to be used as adjuvant therapies in pancreatic cancer [208-211]. However, it was reported that various pancreatic cancer cells respond differently to BET inhibition depending on their molecular subtype [212]. Accordingly, investigation of certain therapies would be more precise if conducted in light of different subtypes rather than in pancreatic cancer patients in general. In case if one therapy is particularly active in a particular subpopulation, these effects can be masked by less than optimal effects in the other populations. Thus, subtyping cancer patients into subgroups who share certain attributes and may respond similarly to certain therapies in patients is highly beneficial. Unlike other cancer types like breast and colorectal cancer, molecular subtyping in pancreatic cancer is still novel with many overlaps and inconsistencies.

\subsubsection{Molecular subtypes of pancreatic cancer}

Molecular subtyping of pancreatic cancer was first conceived by Collisson et al. [213] in 2011. The goal of such subtyping was to reproduce the success in optimizing treatment achieved in breast and lung cancer by identifying different subpopulations. By studying gene expression profiles in various datasets of microdissected tumor material, Collisson et al. identified a gene 
signature made up of 62 genes that clustered the tumors into Classical, Quasi-mesenchymal $(\mathrm{QM})$, and Exocrine-like subtypes. Several pancreatic human and mouse pancreatic cancer cells were found to represent the classical and QM subtypes while the exocrine-like subtype was missing. In spite of microdissection-mediated enrichment of tumor cells, this led the authors to speculate that this subtype is the product of an artifact. Exocrine-like subtypes were so called due to their enrichment with digestive enzymes, QM due their mesenchymal characteristics, and classical were more epithelial-like. GATA binding protein 6 (GATA6) was reported to be enriched in the classical subtype and its knockdown showed a specific dependence of the classical but not the QM subtype on anchorage-independent growth. Additionally, the classical subtype has shown more overexpression of KRAS compared to the QM subtype.

Four years later, this study was followed by a new subtyping approach using virtual microdissection by Moffitt et al. [214] who took into consideration the crucial role played by the stroma. Interestingly, the authors used microarray data for gene expression from normal samples in addition to PDAC tumor samples and metastases. They detected that the stroma usually constitutes half of the tumor sample and that their gene expression profile is clearly distinct from normal tissue. Notably, Moffitt et al. identified two types of stroma, normal which correlates with better prognosis and activated which shows an inflammatory signature and corresponds with worse prognosis. Remarkably, gene expression profiles in tumor cells compared to stroma showed enrichment for mitotic checkpoints and DNA replication. Subtyping of tumor cells uncovered two molecular subtypes called classical, correlating to a better survival rate, and basal-like subtype similar to the basal subtype in breast and bladder cancer. Consistently, gene signatures identifying the basal-like subtype in pancreatic cancer were able to cluster the basal subtype in breast and bladder cancer with a high success rate. Notably, most of the metastatic samples exhibited a basal-like phenotype with an enrichment of keratins. Compared to the Collisson subtypes, the Moffitt clustering was reported to be of higher 
prognostic value strengthened due to the addition of the prognostic tendencies of the normal and activated stroma. The exocrine-like subtype recapitulated the gene signature of samples from adjacent normal tissue while the classical was highly reproduced in the classical subtype reported in Moffitt. Most interestingly, the QM subtype represented a mix of signatures from the basal-like cells and the stroma. Studies in patient-derived xenografts (PDX) confirmed the aggressiveness of the basal-like subtype which showed a higher tumor growth rate. No exocrine-like signatures were detected in the PDX samples and the QM subtypes were detected in mice and human further confirming the contamination of this subtype with stromal cells. Interestingly, the basal-like subtype showed a better response to chemotherapy compared to the classical subtype.

In 2016, Bailey et al. [215] extended the available classification and used 96 bulk pancreatic tumors characterized by having at least $40 \%$ of comprising epithelial cells and studied differential gene expression to identify four different molecular subtypes. Based on similarities to histological phenotypes the four subtypes were called squamous, pancreatic progenitor, immunogenic, and aberrantly differentiated endocrine exocrine (ADEX). These subtypes were validated in an extended set of tumors with different epithelial cellular content. The squamous subtype was characterized by the expression of transcriptional programs related to hypoxia, metabolic reprogramming, and inflammation. Interestingly, the squamous subtype correlated with worse prognosis and expressed high levels of the deltaN isoform of tumor protein p63 (deltaNp63) as a hallmark. The progenitor subtype expressed programs that are characteristic of endoderm fate determination. The immunogenic subtype expressed similar programs in addition to immunogenic programs such as antigen presentation and B cell signaling. While the ADEX subtype also showed enrichment of TFs related to differentiation and development, it exhibited exocrinic and endocrinic features at the same time which is atypical in normal cells. The Bailey classification overlapped with the Collisson classification except for the immunogenic 
subtype which was only defined in Bailey with QM recapitulating squamous, classical showing features of pancreatic progenitor, and the exocrine-like reproducing the ADEX subtype. Such overlap was not discerned upon comparison of the Bailey classification with that of Moffitt but half of the squamous samples were basal-like as well.

The most recent classification was performed in 2017 by the Cancer Genome Atlas Research Network (TCGA) and published in Raphael et al. [216] who took into consideration samples of high and low cellular purity. The authors reported that the basal-like and classical subtypes were recapitulated in high purity samples while the exocrine-like, immunogenic, and ADEX subtypes were exclusively observed in low purity samples implying that these subtypes are observed due to tumor impurities. Raphael et al. used other means for clustering their samples including DNA methylation and long non-coding RNA (IncRNA). Clustering was in concordance with the two subtype classification of classical and basal-like resulting from transcriptional profiling. IncRNA clustering uncovered an enrichment of IncRNA enhancing the transcription of Forkhead Box A2 (FOXA2) and GATA6 in the classical subtype.

These four classifications, though not completely overlapping, currently form the standard of molecular subtyping in pancreatic cancer. These subtypes provide an opportunity for implementation of precision-based medicine for therapeutic management of pancreatic cancer patients $[217,218]$. Studies in organoids, PDX, primary human cell lines and biopsies were able to reproduce the molecular subtyping described in the four standard studies (Collision, Moffitt, Bailey, and Raphael) [219-223]. Screening of various inhibitors in different lines representing various subtypes uncovered that distinct subtypes show different dependencies and vulnerabilities [224, 225]. Additionally, studies in pancreatic cancer patients revealed that molecular subtype can correlate not only with prognosis but also with response to chemotherapy [226]. Accordingly, molecular subtyping of pancreatic cancer presents a highly important milestone in the optimization of therapy for pancreatic cancer patients. 
Given the worse prognosis of the squamous/basal-like subtype, understanding the molecular mechanisms taking part in this particular subtype is of the utmost importance. We previously mentioned that high expression of deltaNp63 is a feature of the squamous phenotype. Its role and its functions are discussed in the following section.

\subsection{DeltaNp63 in cancer}

p63 along with the known tumor suppressor p53 and tumor protein P73 (p73) are transcription factors comprising the p53 family [227]. At the time of its discovery, p63 was presumed to play a comparable role to p53 due to their structural similarity [228]. One of the most known roles of p53 is stress-induced initiation of apoptosis [229]. p63 has two major isoforms, the first isoform is the transactivation domain-containing p63 isoform (TAp63) and the second isoform is called the transactivation domain-lacking p63 isoform (DeltaNp63/ $\Delta \mathrm{Np63}$ ) [228]. Each of these has 3 minor isoforms that differ at the C-terminal domain named $\alpha, \beta, \gamma$, respectively. The structure of p63 isoforms compared to p53 and p73 is depicted in Figure 11 (modified from the first paper to clone and identify p63 in 1998, Yang et al. [228]).

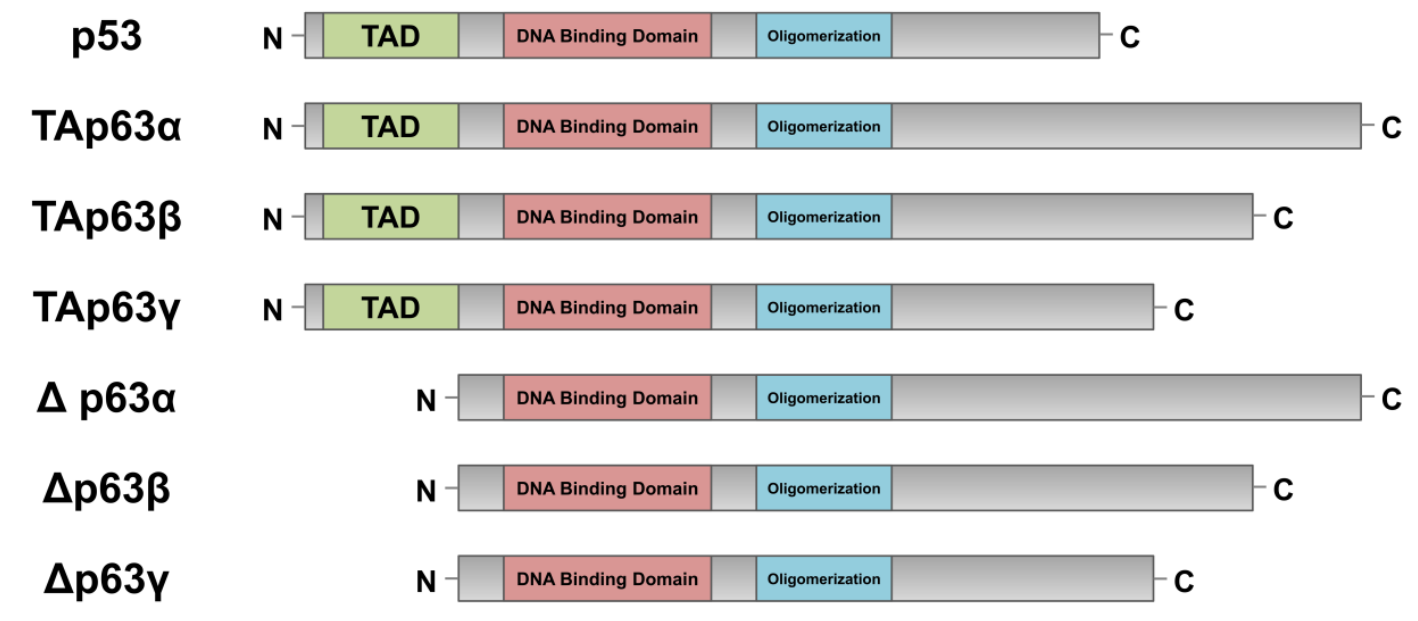

Figure 11 : Schematic structure of the isoforms of p63 compared to p53. (modified from Yang et al.) 
Due to the lack of the transactivation domain in deltaNp63 and its inability to activate p53 target genes in contrast to TAp63 in a reporter assay conducted in osteosarcoma cells, Yang et al. concluded that deltaNp3 is the dominant negative form and reported that it is usually expressed in 200 basal and squamous cells. Consistently, knocking out p63 in mice resulted in deformed or missing limbs and global effects on epithelial tissues [230, 231]. In addition, deltaNp63 was reported to be highly expressed in cancers and molecular subtypes of cancer that are squamous or basal in their nature such as breast, head and neck, lung, and esophageal carcinoma [232-235]. Interestingly, deltaNp63 expression was found to correlate with aggressiveness in salivary glands tumors and with clinical stage and metastasis in oral squamous cell carcinoma [236-238]. Knockdown of deltaNp63 led to increased sensitivity to chemotherapy in head and neck and lung squamous carcinomas [232]. A report in 2005 observed the upregulation of deltaNp63 in pancreatic cancer [239]. DeltaNp63 garnered more attention in the pancreas when Bailey et al. revealed its high implication in the squamous phenotype [215].

Recently, p63 was identified to drive expression of the IncRNA LINC01503 through a super enhancer region [240]. LINC01503 was found to enhance proliferation and invasiveness of esophageal carcinoma cells through activating the mitogen activated protein kinase (MAPK) and phosphatidylinositol 3-kinase and Protein Kinase B (PI3K/AKT) pathways. Interestingly, knockdown of this IncRNA not only decreased proliferation and anchorage-independent growth, but also sensitized cells to inhibitors targeting the pathways it activates. p63 was also reported to drive keratinocyte differentiation with the help of the histone methylase MLL4 through distal enhancers [241]. So far, the mechanisms by which deltaNp63 drives its effects and promotes malignancy are not well-defined. Future studies will extend our knowledge about this important TF and might provide a new therapeutic target in subtypes of cancer that are associated with worse prognosis. 


\subsection{Aims and scope of this project}

This project focuses on investigating the role of distal regulatory elements in driving aberrant gene transcriptional regulation in malignancy-related contexts. In particular, emphasis is given on elucidating the role of super enhancers, validating their importance, and defining major drivers in this subcategory of distal regulatory elements. This is done with the goal of not only shedding light on molecular mechanisms underlying development and progression of malignant disease, but also uncovering new targets for therapies and biomarkers for prognosis and therapeutic effectiveness.

This project includes a series of four studies presented in this thesis as two publications in addition to two manuscripts. The first publication (Chapter 2) aims to validate the importance and alleged high efficacy of super enhancers. This is tested in a clearly-defined system where enhancers and their drivers are clearly elucidated. Accordingly, a pattern for gene transcriptional regulation control via enhancers and/or super enhancers can be concluded from this system (estrogen and BRD4 in breast cancer). Such patterns can be tested in other contexts which could lead to its validation in various systems or the finding of new system-specific patterns. These models can then be manipulated and leveraged in reversing aberrant transcription in malignant diseases.

As a clear superiority of super enhancers in breast cancer was not observed, we extended our study to include the particularly aggressive pancreatic cancer. In the second publication (Chapter 3), our main objective is to evaluate the role of targeting enhancers and super enhancers in pancreatic cancer via the epigenetic modulators, BET and HDAC inhibitors. Additionally, we use publicly available data for transcription factor localization throughout the genome to identify context-dependent enhancers in different malignancies. Moreover, we aim to 
find the mechanism by which BET and HDAC inhibitors work synergistically in pancreatic cancer.

Subsequently, we endeavored to focus on the role of super enhancers in pancreatic cancer. Thereby, we uncovered the transcription factor deltaNp63 as a major driver. Accordingly, in the first manuscript (Chapter 4), our purpose is to understand the molecular mechanisms by which deltaNp63 drives a distinct molecular subtype via super enhancers. We also intend to uncover transcription factors that co-operate with deltaNp63 in molecular subtype development. Additionally, we aim to better characterize the currently defined molecular subtypes of pancreatic cancer and validate the implication of deltaNp63-driven super enhancers using various systems.

Finally, we aim to extensively study the role of enhancers in the development of resistance to chemotherapy in pancreatic cancer. Thus, we aim to identify changes in the pattern of gene transcriptional regulation by enhancers in the second manuscript (Chapter 5). Accordingly, we identified BET inhibitors as a potential sensitizer for chemo-resistant pancreatic cancer. 


\title{
2. Publication I
}

\section{CHAPTER 2}

\section{Super enhancers - new analyses and perspectives on the low hanging fruit}

\author{
Feda H. Hamdan ${ }^{1}$ and Steven A. Johnsen ${ }^{1,2}$ \\ ${ }^{1}$ Department of General, Visceral and Pediatric Surgery, University Medical Center Göttingen, 37075 \\ Göttingen, Germany \\ 2 To whom correspondence should be addressed
}

\section{Keywords}

BRD4, super enhancers, typical enhancers, enhancer RNA, ERa activation, transcriptional regulation, ROSE algorithm

'This is the authors accepted manuscript of an article published as the version of record in Transcription (c) Taylor \& Francis https://doi.org/10.1080/21541264.2017.1372044'

Transcription. 2018;9(2):12t3-130. doi: 10.1080/21541264.2017.1372044. Epub 2017 Nov 3.

Contributions: Bioinformatic analysis was done by F.H.H. Figure 1 was made by F.H.H. and Figure 2 by F.H.H. and S.A.J. F.H.H. and S.A.J conceived the ideas for this project and wrote the manuscript. 


\subsection{Abstract}

Significant attention has recently been given to a class of enhancers termed "super enhancers", while implying that "typical enhancers" are less important. In this report, we examine criteria for identification of super enhancers and address the need to evaluate the differences between BRD4-occupied "typical" and "super" enhancers.

\subsection{Introduction}

Bromo- and extra-terminal (BET) domain proteins, most notably BRD4, represent an important class of epigenetic regulators with a particularly strong relevance for various human diseases including cancer, fibrosis, heart failure, etc [242]. BET proteins function as "epigenetic readers" which recognize acetylated lysine residues on both histone and non-histone proteins and serve to promote target gene transcription [26, 243]. A number of recent studies from our group and others have revealed a particular importance of BET proteins in the control of gene expression via distal enhancer regions. Notably, the discovery of small molecule inhibitors (BETi) which block the binding of BET proteins to chromatin [244, 245] have led to an explosion of research into the biology of these proteins and ultimately to numerous early phase clinical trials to test their efficacy in the treatment of various malignancies [246]. However, a major hurdle that remains is the ability to accurately predict the biological and transcriptional effects of BETi based on transcriptional and/or genome-wide occupancy profiles. We have recently shown that BRD4 plays a significant role in the transcription of lineage-specific genes in human fetal osteoblasts, mainly through localization with different transcription factors at enhancer regions [99]. Similarly, we showed that the majority of BRD4-enriched regions in mammary epithelial cells were associated with putative enhancers [247]. Remarkably, more than one-third of epithelial-to-mesenchymal transition-related genes showed an enrichment of BRD4 at an 
adjacent distal region. Overall, enhancers emerge as a common and decisive mechanism in BRD4-mediated regulation of gene transcription.

Since the term "super enhancer" (SE) was coined [123, 124] a significant emphasis has been placed on this class of enhancers while implying that other "typical enhancers" (TEs) are of less importance. However, the exact characteristics that render an enhancer "super" and not "typical" are still poorly defined and fairly arbitrary. In our previous work $[99,247]$, we identified potential enhancers important for mediating tissue-specific BRD4 activity using a differential occupancy approach, independent of "super" classification. This work resulted in important insights into BRD4 function at putative distal enhancer regions, which could be functionally verified in both systems. In this report, we sought to examine the potential relevance of "super enhancers" by following different approaches to identify BRD4-dependent "super enhancers" with the goal of testing if they indeed represent a special class of regulatory elements with a particularly strong influence on gene regulation. Furthermore, we examined if we can more meticulously identify these presumed highly efficient regulatory units that can be optimally harvested to effectively predict the effects of their pharmacological perturbation on gene transcription.

\subsection{Results and Discussion}

\subsubsection{Super vs. Typical Enhancers: What is so bad about being typical?}

In general, SEs are considered to be large clusters of regulatory elements that are highly occupied by transcription factors and have high potential to activate transcription of their target genes [248, 249]. It was recently found that single nucleotide polymorphisms (SNPs) within BRD4-enriched SEs increase the chances of the development of breast and prostate cancers [250]. BRD4 was also reported to occupy the Colon Cancer Associated Transcript 1 (CCAT1) 
super enhancer, thereby enhancing the expression of the nearby MYC oncogene and accounting for the anti-proliferative effects of BET inhibition in colorectal cancer [251]. Cell lines that do not include a super enhancer driving MYC expression have been reported to be largely unresponsive to BET inhibition. Deleting the upstream super enhancer region of Myc in mice specifically affected its expression in tissues like the colon and prostate and led to a partial loss of the mammary tumorigenic phenotype in these mice [252]. While these and other studies validate the increased interest in SEs, this might inadvertently lead to the disregard of important "typical" enhancers that can significantly mediate the effects of BRD4 and may efficiently be exploited in its perturbation.

In order to evaluate the individual contributions and importance of SEs and TEs, we used our previously published dataset (GSE55921/2) in which we examined the importance of BRD4 in controlling ERa-activated gene transcription in ER-positive MCF7 breast cancer cells [152]. We specifically chose this system as it ideally suits our analyses due to the rapid and direct effect of ligand (estrogen) binding to the estrogen receptor-alpha (ERa) and the robust effects on transcriptional activation. Additionally, ERa activity in this system is highly dependent on BRD4 demonstrated by the observation that $83 \%$ (126 out of 152) of the genes that are significantly upregulated by estradiol (E2) treatment ( $>2$ folds, q-value $<0.05)$ are significantly downregulated upon knockdown of BRD4 ( $<1.4$ folds, q-value $<0.05$ ). We hypothesized that if SEs play a more prominent role in mediating BRD4 effects than TEs, the 126 genes downregulated upon knockdown of BRD4 will show a higher correlation with BRD4-driven "super" than "typical" enhancers. 
In order to identify putative enhancers, we used genome-wide occupancy data for histone 3 acetylated on lysine 27 (H3K27ac) in E2-treated MCF7 (GSE40129) [253] and identified SEs based on their BRD4 signal intensity using the Ranking of Super Enhancers (ROSE) algorithm $[123,124]$, the most commonly utilized approach to identify SEs. In this way, we were able to identify 324 super enhancer regions [Figure 12A] and 24,427 TEs. To selectively narrow enhancers to those related to ERa function, we intersected them with ERa peaks followed by intersection with BRD4 peaks to select only for TEs that are occupied by both ERa and BRD4 and eliminate any regions where a direct effect of estrogen and BRD4 could not be established. Subsequently, we extracted the nearest genes in a window of $500 \mathrm{~kb}$ using the BETA-minus version 1.0.0 on the Galaxy platform and utilized CTCF boundaries to filter the genes.

Interestingly, there were twice as many genes associated with TEs compared to SEs which were downregulated in response to BRD4-depletion (Figure 12A, Venn diagram). This indicates that TEs likely play a highly significant role in BRD4-mediated gene transcription regulation and should not be overlooked. On the other hand, SE-associated genes included major players in estrogen response, including GREB1, TFF1, XPB1, in addition to the widely known BRD4 target, MYC. This may imply that master regulatory genes show a tendency to be more related to SE-mediated regulation, possibly due to more efficient control by a cluster of enhancers rather than by individual ones. In general, we failed to find significant differences between SEand TE-associated genes in their tendencies for downregulation upon BRD4 knockdown (Figure $12 \mathrm{~A}$, box plot). 


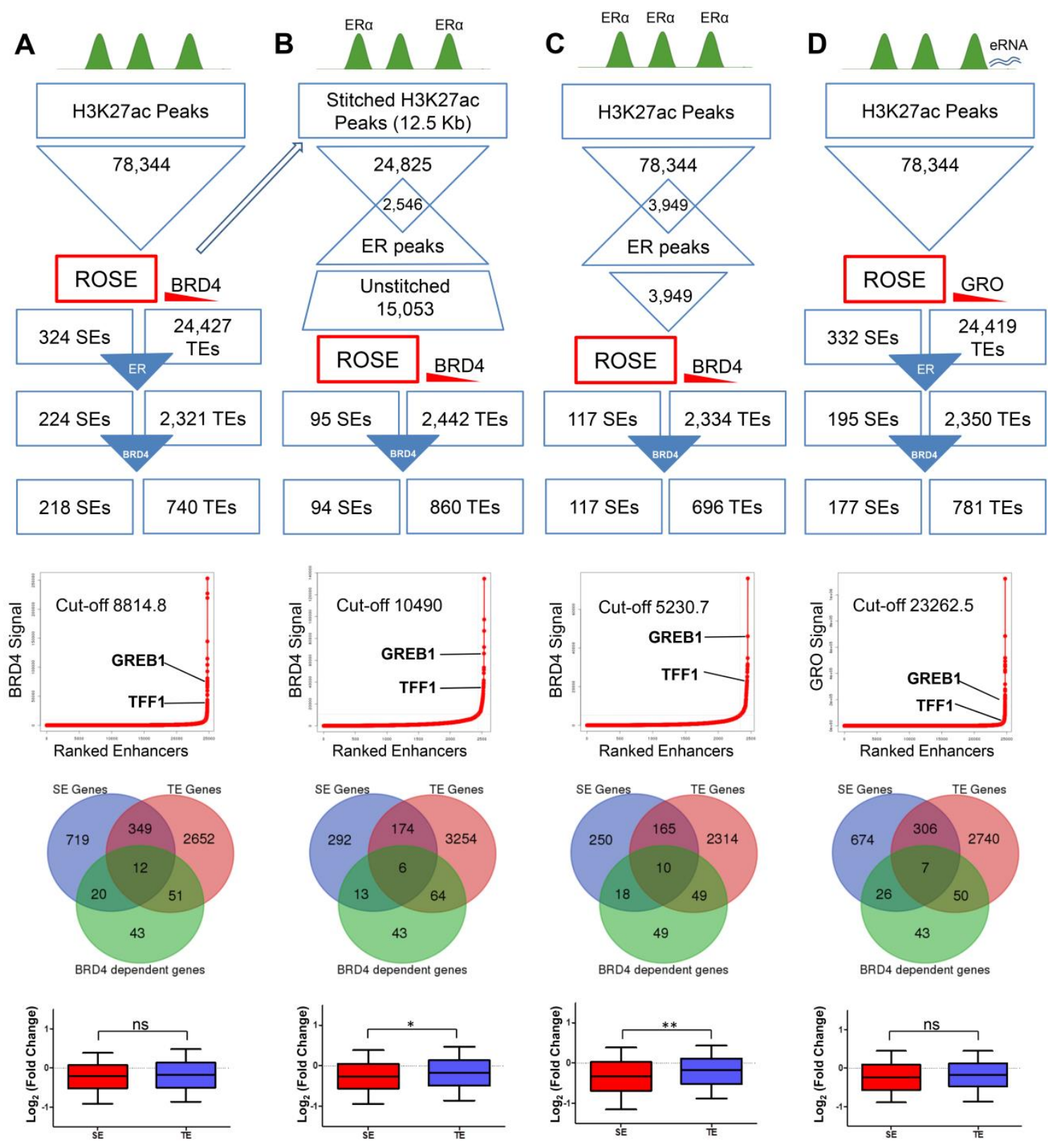

Figure 12 : Different approaches followed to identify super enhancers using the ROSE algorithm. (A-D) Feeding ROSE with H3K27ac peaks and ranking regions based on BRD4 signal (A), only H3K27ac peaks that co-localize with or are adjacent to ERa (B), H3K27ac peaks that only co-localize with ERa (C) or H3K27ac peaks and ranking regions based on GRO-seq signal (D). In each panel, the specific workflow of the SE and TE identification is shown, followed by the ROSE output showing GREB1 and ESR1 approximate ranks as examples. Venn diagrams show the overlap of SE- and TE-associated genes with BRD4-dependent genes (126 genes that are upregulated by E2 treatment and significantly downregulated by BRD4 treatment). At the bottom of each panel, a box plot shows the general tendency of regulation of SE- or TE-associated genes upon knockdown of BRD4. 


\subsubsection{Picking the petals of ROSE: an enhancer is super, an enhancer is not super...}

The ROSE algorithm is currently the gold standard for the identification of super enhancers in addition to other scripts which follow the same rationale. Certain regions are used as an input for ROSE and stitched together if they are less than the default $12.5 \mathrm{~kb}$ apart. Subsequently, stitched regions are ranked according to the intensity of the signal of a chosen transcription factor or cofactor (e.g., Mediator, BRD4, etc.). Super enhancers are identified as those for which the signal is particularly high and surpasses a specific cut-off point. The regions that are close to the transcriptional start site can also be disregarded with a default threshold of $2500 \mathrm{bp}$. Thus, the process of the identification of super enhancers can be affected by different variables.

A deciding factor for the identification of super enhancers is the input regions that are inserted into ROSE. They form the general population among which SEs are selected. Naturally, the higher the number of the regions, the more "difficult" it is for a certain region to rise above the specific cut-off point and be classified as a SE. Super enhancers comprise a luring target not only due to their high activation potential but more importantly due to their specific and dependent mediation of gene regulation. As we were specifically interested in the potential role of BRD4-occupied super enhancers in the context of ERa function, we intersected our enhancer regions with ERa peaks after the identification of SEs by ROSE. To check the effect of limiting the regions before running ROSE, we used H3K27ac peaks that are co-localized or adjacent to ERa peaks as input for the algorithm. As expected, this approach reduced the number of SEs to 95 (Figure 12B). Interestingly, as the processed region numbers were less than $20 \%$ of those used in the standard way, the cut-off point increased and ranks of estrogen-regulated genes improved, which could be expected since only estrogen-related regions were taken into account. Conversely, the number of SE-associated genes that are dependent on BRD4 
decreased, while still including the same key ERa target genes defined previously (Figure 12B, Venn diagram). Remarkably, this approach started to reveal a more significant dependence of SE-associated genes on BRD4 compared to TE-associated genes.

Overall, by inserting a simple focused "tuning" to ROSE, we were able to more precisely select for a predictive subgroup of enhancers. Ultimately, our goal is to identify subgroups of enhancers within a larger population of regions, which may more precisely predict the effects of BETi treatment. Altogether, the ranking of enhancers by ROSE is highly dependent on the number and nature of the regions which are inserted as input into the algorithm in addition to the stitching threshold. As such, the ranking should be used as a tool rather than a goal in identifying highly functional transcriptional regulatory subunits. This can support the rationale to limit the tested regions in the hope of identifying meaningful mediators regardless of the fact if their "super" rank is ultimate (from all possible regions) or regional (from specifically picked regions).

\subsubsection{Super enhancer subcomponents: one for all or all for one?}

While super enhancers are generally considered to comprise very broad H3K27ac peaks, chromatin accessibility can frequently provide an opportunity to narrow down the regions in order to decipher individual components of SEs. Usually, these enhancers include multiple peaks of chromatin accessibility (e.g. from DNase or ATAC-seq), where chromatin is accessible to transcription factors. This raised the question if these clusters work in an additive or synergistic manner [248]. Recent developments in ground-breaking genome editing techniques have enabled scientists to investigate the contribution of individual subcomponents of certain SEs. Mosaic-seq uses CRISPR-mediated deletion of certain clusters of a super enhancer in order to evaluate their role in gene activation. This revolutionary method has revealed that in the 
case of the $\beta$-globin locus control region (LCR), one cluster within this super enhancer region is largely responsible for the activation of its target gene, HBG2 [254]. Conversely, deletion of individual regulatory elements of the $\alpha$-globin SE in mice showed no significant preference of any of the SE subcomponents [255]. Concordantly, the high activation potential of the SE associated with the mammary-specific Wap locus was partially dependent on each of the singular components [256].

Accordingly, an important question arises of whether SEs comprising multiple components solely related to ERa can have greater effects on gene regulation than other SEs which include ERa in only one or a few of its components. For this purpose, we performed a highly biased analysis by using only H3K27ac peaks that intersect with ERa peaks as input for ROSE (Figure 12C). This approach significantly decreased the cut-off point of SE identification to half of that compared to our previous approach when we allowed for all components independent of colocalization with ERa. Surprisingly, this increased the numbers of SEs to 117, but did not increase the number of BRD4-dependent SE-associated genes. SE-associated genes in this case also showed more significant downregulation by BRD4 knockdown (Figure 12C, box plot).

To identify whether SEs affect more prominent regulators than TEs, we used the GREAT analysis tool and performed gene ontology analyses on the two nearest genes in a $500 \mathrm{~kb}$ window to SEs and TEs [257]. To correct for the large difference in numbers between the SEand TE-associated genes, which may lead to a profound bias when calculating significance, we intersected the TE- and SE-associated peaks with DNase-seq from E2-treated MCF7 (GSE33216) [258]. Only SE regions defined in the most biased approach (Figure 12C), where we intersected the H3K27ac peaks with ERa peaks, showed significant association with ontology terms related to estradiol, hormone, and estrogen response, while TE regions did not comprise any significant ontology terms related to estrogen or hormones. Both SE and TE 
regions failed to show meaningful gene associations using the other previous approaches (data not shown).

Overall, our observations imply that SEs can include subcategories depending on the transcription factors and niche of each subcomponent. Overall, not all SEs will necessarily follow the same rule. As we learn more about SEs, we will be able to better understand the impact of their various individual components and identify which are additive and which are synergistic. Currently, it is of high interest to test if the activation of any element of a specific SE will be sufficient to activate a gene that is otherwise not active. To do so, a driving transcriptional cofactor such as BRD4 or p300 can be tethered to specific regions using nuclease-deficient Cas9 (dCas9) in a system where the SE is not active [model in Figure 13]. This approach has been successfully used to ectopically induce DNA methylation [259] and induce acetylation of enhancer regions.[260] Thus, using this approach will enable us to test the effects of targeting different transcriptional regulatory proteins to specific loci (e.g. in TE or SE) in order to derive conclusive data that will help in identifying the dependencies of enhancers and further validate their effects.

\subsubsection{Enhancer RNAs: whether bystanders or effectors, they are anyways a great help}

Enhancers were recently shown to frequently produce bi-directional non-coding, generally shortlived transcripts referred to as enhancer RNAs (eRNA), which appear to be involved in inducing nearby target genes [103]. It was reported that eRNAs near E2 activated genes are upregulated upon E2 treatment and function as stabilizers for the looping of enhancers with the promoters of ERa target genes [261]. Conversely, a recent study has found that eRNAs rarely co-localize with an enhancer-promoter-loop associated with active transcription [262]. Hah et al. showed 
that the majority of macrophage SEs in mice produced eRNAs in contrast to only one third of TEs [263]. To date, it still remains unclear whether eRNAs are a mere by-product given their presence in the midst of transcriptional factories or actual effectors that are important central regulators of gene transcription.

In order to examine this further, we used publically available Global run-on (GRO-seq) (GSE43836) [264] data for E2-treated MCF7 cells to test whether using this information to predict super enhancers results in similar findings to BRD4 ChIP-seq data. Remarkably, we were able to identify a similar number of SEs by ranking them based on GRO-seq, rather than BRD4 (Figure 12D). Out of the 177 SEs that co-localize with ERa and BRD4, 106 enhancers were contained within the 218 genes identified using the BRD4 signal, suggesting there is a substantial, but only partial overlap in the results obtained using the two approaches. Notably, using GRO-seq to classify SEs increased the number of BRD4-dependent genes that are related to SEs and included other estrogen responsive genes like HSP80 and RAB31. However, it did not affect the tendency of downregulation following BRD4 depletion between SE- and TEassociated genes, which stayed insignificant when using all the H3K27ac regions (Figure 12D, box plot). Whether eRNAs are bystanders or effectors, in any case, they can be highly effective predictors of enhancers and may possibly be used in place of the most commonly-used transcription factors like MED1 or BRD4 when identifying and analyzing enhancer regions. 
A No enhancer activation

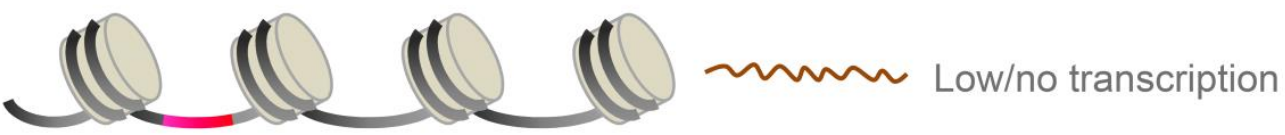

B Super enhancer activation by transcription factors

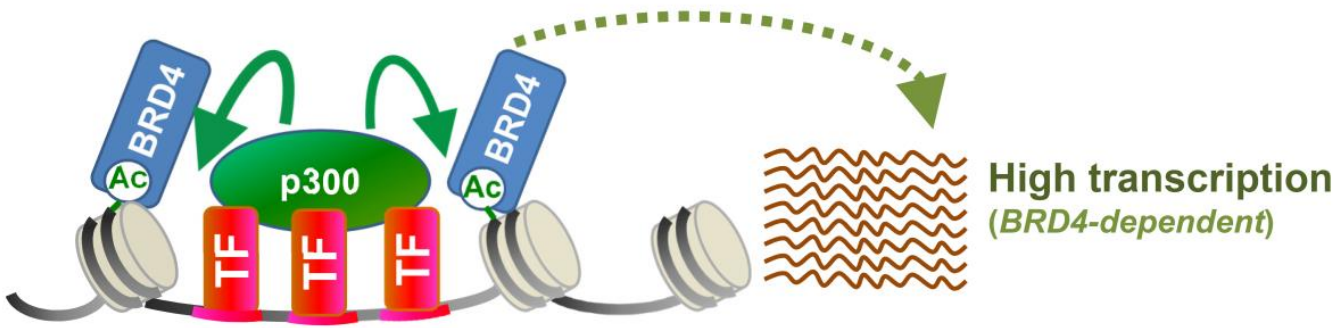

C dCas9 tethering of BRD4 to inactive super enhancers

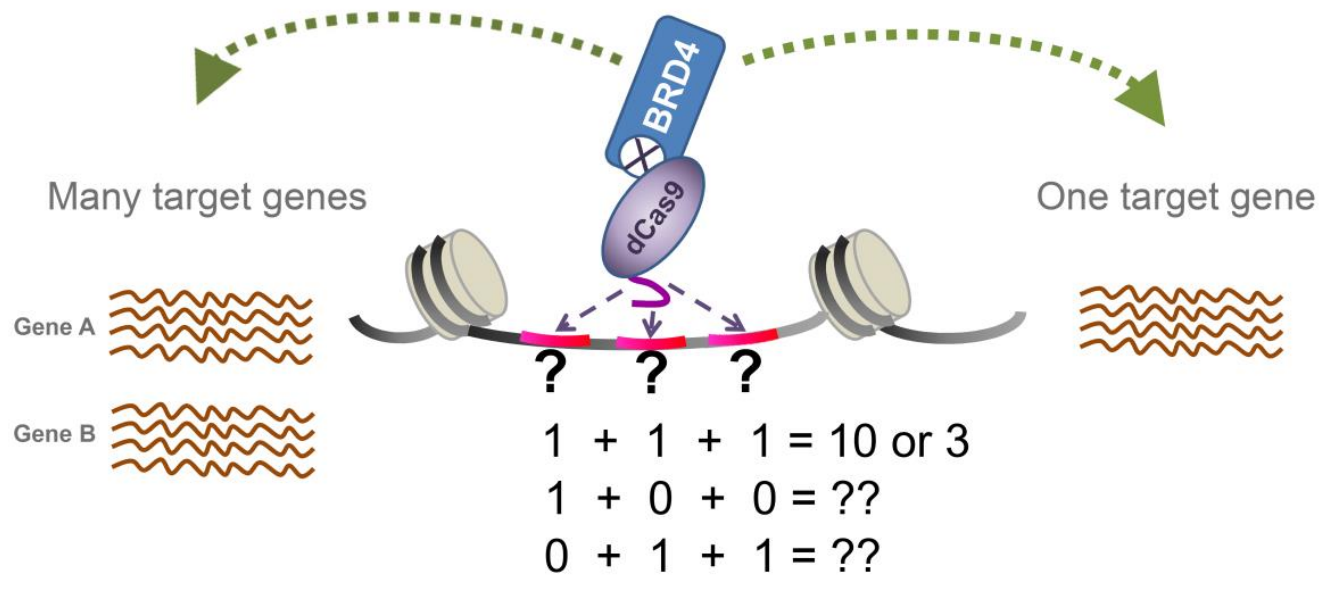

Figure 13 : Tethering of BRD4 to enhancer regions via nuclease-deficient Cas9 (dCas9) can provide causative information about the contribution of individual components of (super) enhancers. (A) An inactive enhancer region is shown which displays no significant transcriptional activity. (B) In the case of an active super enhancer, different or identical transcription factors can lead to the recruitment of other activators, in this model p300-mediated acetylation of histones serves to promote recruitment of BRD4. This then leads to increased transcription of the target gene. (C) Tethering of BRD4 in an acetylation-independent manner to subcomponents of a super enhancer can validate the contribution of each element to the enhancer. By tethering BRD4 to one region, we can verify if one of the elements are sufficient to account for the high activation potential of the super enhancer $(1+0+0=10)$. We can also evaluate the loss of the one component $(0+1+1=5)$ and thus verify if this certain super enhancer works synergistically $(1+1+1=10)$ or in an additive manner $(1+1+1=3)$. Finally, tethering can also provide causative data to identify target genes of specific enhancers and determine the complex interactions between these enhancers and their target gene(s). 


\subsection{Conclusions}

In this report, we sought to challenge the current approach for identifying functionally important distal enhancer regions and make a simple comparison between SEs and TEs. We aimed to shed light on the distinctive roles of TEs and SEs since many researchers remain skeptical about this classification, at least in part due to the lack of a clear definition. Irrespective of categories, increasing our understanding of enhancer mechanisms of action and dependencies will not only provide us with novel approaches to predict the effects of epigenetic manipulation of enhancer function (e.g., through BETi), but will also endow us with unprecedented potential to identify and develop new strategies to specifically manipulate gene expression in different contexts and diseases. We observed that, irrespective of the approach for SE identification, a high number of genes showed regulation by TEs, which underscores the importance of studying the role of all enhancers rather than focusing only on the SE subcategory. While the frequent association of SE with master regulators may justify the recent interest in their characterization, SEs may simply be the "low hanging fruit" of enhancers that can prejudice our investigations and prevent us from identifying other important regulatory elements that can have significant impacts and effects. Instead, a differential occupancy approach as we recently described [99, 247], may provide a more effective approach to identify functionally important enhancers and their underlying transcription factor networks.

\subsection{Methods: Analysis}

Fastq files were mapped to the hg19 genome using BOWTIE2/2.2.6 with very sensitive end-toend options. Peaks were called using MACS2/2.1.0 without building the shifting model and with input peaks as background. Narrow peaks were called for ER a and broad peaks with a cut-off of 0.05 were called for BRD4 and H3K27ac. RNA-seq data were mapped by TOPHAT/2.1.0 to the hg19 genome and differential analysis was performed using CUFFLINKS/2.2.1. GRO-seq 
(SRR653425/6) was mapped using BOWTIE2/2.2.6 and bigwig files were generated using DEEPTOOLS/2.4.0 with ignoring the duplicates and extending for 200bp. Box plots were generated using GraphPad Prism 5 with significance calculated using the Mann-Whitney test.

\subsection{Disclosure of interest}

The authors report no conflict of interest.

\subsection{Acknowledgements}

The authors would like to thank Xin Wang, Madhobi Sen, and Matthias Dobbelstein for valuable discussions. 


\title{
3. Publication II
}

\section{CHAPTER 3}

\section{Epigenetic Targeting of Aberrant Transcriptional Modulation in Pancreatic Cancer}

\author{
Feda H. Hamdan* and Steven A. Johnsen* \\ Department of General, Visceral and Pediatric Surgery, University Medical Center Göttingen, 37075 \\ Göttingen, Germany \\ ${ }^{*}$ Correspondence: fhamdan@gwdg.de (F.H.H.); steven.johnsen@med.uni-goettingen.de (S.A.J.); Tel.: \\ $+49-551-39-13711$ (S.A.J.)
}

\section{Keywords}

Bromodomain and Extra-terminal (BET) inhibitors; histone deacetylase (HDAC) inhibitors; pancreatic cancer; aberrant transcription; enhancers; transcription factors; distal regulatory elements; MYC; Forkhead Box A1 (FOXA1); Bromodomain containing protein 4 (BRD4)

This publication is a freely accessible at www.mpdi.com

Epigenomes 2018, 2(2), 8; https://doi.org/10.3390/epigenomes2020008

Contributions: Bioinformatic analysis was done by F.H.H. F.H.H. and S.A.J. conceived the ideas for the figures and the project and wrote the manuscript. 


\subsection{Abstract}

While the mortality rates of cancer are generally declining, pancreatic cancer persists to be an exception with a 5-year-survival rate of less than $7 \%$. Late diagnosis and resistance to conventional therapies contribute to high mortality rates in spite of the remarkable recent advances in cancer management and research. Consequently, there is an urgent need to find new and unconventional therapeutic targets to improve prognosis and survival of pancreatic cancer patients. In this review, we discuss the transcriptional effects of the most widely used epigenetic inhibitors in pancreatic cancer focusing on Bromodomain and Extraterminal domain (BET) and Histone Deacetylase (HDAC) inhibitors, which are currently highly promising therapeutic options. We suggest that these inhibitors can be better utilized at lower doses which exploit their transcriptional modulatory effects on pancreatic cancer transcriptional programs directed by specific factors such as MYC and Forkhead Box A1 (FOXA1), rather than simply based on their anti-proliferative effects. This approach can potentially help avoid the intolerable adverse events frequently elicited by the use of these treatments at higher doses. In particular, we underscore the crucial role of distal regulatory elements in mediating the specific effects of these epigenetic inhibitors and propose using them in a more selective and prudent manner.

\subsection{Introduction}

While the mortality rates of cancer are generally declining, pancreatic cancer persists to be an exception with a 5 -year-survival rate of less than $7 \%[153,265]$. Late diagnosis and resistance to conventional therapies contribute to high mortality rates in spite of the remarkable recent advances in cancer management and research [246]. Consequently, there is an urgent need to find new and unconventional therapeutic targets to improve prognosis and survival of pancreatic cancer patients. 
In addition to various genomic mutations, such as KRAS and TP53, that play a role in the pathophysiology of pancreatic cancer, other mutations and signaling pathways play an equally important role by affecting transcription of entire subsets of genes, irrespective of genomic sequence [215, 246, 265-269]. Epigenetic pathways affect transcription either via modulation of histone modifications which can be activating or silencing, DNA methylation-mediated silencing, non-coding RNAs, and alteration of chromatin accessibility [270]. This meshwork provides the cells with various tools that can dramatically affect its transcriptome without the need to induce any irreversible changes at the level of the genome. Moreover, in contrast to the permanent and largely "all or nothing" effects of genomic mutations, modulation of the epigenome allows for more subtle, reversible changes in genome regulation.

Epigenetics represents a promising target in pancreatic cancer for various reasons. Firstly, epigenetic modifications are mainly mediated by enzymes and proteins whose activity can (at least in principle) be targeted by small molecule inhibitors. Secondly, many epigenetic pathways were found to be deregulated in pancreatic cancer, suggesting a crucial role of epigenetic regulation in this malignancy [215, 266-268]. Additionally, pancreatic cancer, among others, was found to be addicted to the activation of aberrant transcriptional programs that not only drive the development and progression of cancer, but are also crucial for the maintenance of detrimental malignant characteristics such as metastasis and chemoresistance [271, 272]. Epigenetic pathways were found to be major drivers of the perturbations of such programs in a highly intricate and context-specific manner [273]. Due to its tremendous pliability, epigenetic modulation provides an optimal tool to be hijacked in cancer development and progression creating a specific dependence of cancer cells on these pathways. Accordingly, many epigenetic inhibitors are currently under investigation for the treatment of numerous malignancies, including pancreatic cancer [246, 274-276]. However, results from these studies so far have been unexpectedly modest and, in some cases, these inhibitors were associated 
with intolerable toxicities. Many of these studies use epigenetic inhibitors, whether alone or in combination, in a method akin to other more conventional drugs with defined targets such as chemotherapy and monoclonal antibodies. On the other hand, epigenetic inhibitors target transcriptional regulation in a complex and unconventional manner and this should be taken into consideration when investigating these drugs.

Numerous reviews have deeply discussed and extensively summarized the recent advances of targeting epigenetics in cancer, in general, as well as in pancreatic cancer in particular [246, $270,277-282]$. In this review, we focus on the transcriptional mechanisms of the most widely used epigenetic inhibitors in pancreatic cancer focusing on Bromodomain and Extraterminal (BET) and Histone Deacetylase (HDAC) inhibitors, which represent promising therapeutic options. We suggest that these inhibitors can be better utilized for their transcriptional modulation, rather than solely on their anti-proliferative effects, which can lead to intolerable adverse events. Moreover, we underscore the crucial role of distal regulatory elements in mediating the specific effects of these epigenetic inhibitors and propose using them in a more selective and prudent manner.

\subsection{Bromodomain and Extraterminal Inhibitors in Pancreatic Cancer}

The BET family of proteins consists of BRD2, BRD3, BRD4, and the testis-specific BRDT [123]. All BET family members contain two conserved bromodomains which enable them to recognize acetylation marks on the chromatin, in addition to an extraterminal domain which enables interactions with other proteins $[26,199]$. The BET family has attracted much attention due to its significant role in gene transcription regulation in addition to its implication in the development of the particularly aggressive Nuclear protein in Testis (NUT) midline carcinoma, which is characterized by the presence of a BRD4-NUT fusion gene and arises in the mediastinum, head, and neck of typically young patients [245, 283-285]. This aberrant fusion gene includes 
the two bromo- and extraterminal domains of BRD4, in addition to a histone acetyltransferase (HAT) binding domain from the NUT gene. Thus, the fusion gene has the ability to aberrantly activate large chromatin domains [273]. The synthesis of the prototype BET inhibitor JQ1, which competitively binds to the acetylation-recognizing hydrophobic pockets in all BET members, has marked an explosion in the number of studies investigating the role of BET family members in gene transcription regulation due to their promising anti-proliferative effects in different cancer types $[245,286]$. However, many aspects are still unknown about the role of these factors in driving transcriptional activation and the best way to leverage their context-specific effects.

\subsubsection{Role and Effects of Bromodomain and Extraterminal Inhibitors in Pancreatic Cancer}

Interestingly, a general screen for limiting epigenetic regulators in pancreatic cancer identified two members of the BET family, BRD2 and BRD3, as major drivers in pancreatic cancer growth and progression [287]. In pancreatic cancer cell lines, BET inhibition exerts anti-proliferative effects by selectively targeting inflammatory and oncogenic pathways [196]. This effect was also observed even in pancreatic cells with chemotherapy-resistant and highly migratory phenotypes [210]. Concordantly, BET inhibitors were found to decrease tumor growth in patient-derived xenografts by attenuating inflammatory pathways in cancer cells and their associated fibroblasts [209, 287, 288]. Recently, Andricovich et al. [212] showed that pancreatic cells deficient for the lysine demethylase, KDM6A, are more sensitive to BET inhibition due to the activation of BETdependent super enhancers. Moreover, the MYC proto-oncogene, perhaps the best known target for BET inhibition, was successfully used to subgroup 55 pancreatic cancer patientderived xenografts based on its expression and accurately predicted sensitivity to BET inhibition via apoptosis with high expression of $M Y C$ correlating with more BET dependence [289]. 
Thus, it has become very clear that BET inhibitors have a perceptible anti-proliferative effect in pancreatic cancer and display a very promising potential as highly effective and selective therapeutic agents. These inhibitory effects may largely be due to BET members being crucial for driving aberrant transcriptional programs and can be heightened via specific deficiencies which lead to increased transcriptional dependence on BET family members. This supports the current efforts to identify certain subgroups of patients who may be more responsive to BET inhibition. However, it is important to note that the effects of BET inhibition are varied and highly specific for different tumor types and subgroups.

\subsubsection{Bromodomain and Extraterminal Inhibition and Metastasis in Pancreatic} Cancer

Metastasis is a major contributing factor to the very poor prognosis of the majority of pancreatic cancer patients [290]. Consequently, therapeutic agents that attenuate and/or prevent metastasis can be of extreme benefit to patients. Recently, it was uncovered that pancreatic mouse organoids from metastatic pancreatic ductal adenocarcinomas show a marked reprogramming in their enhancer landscape compared to organoids originated from normal pancreata, early neoplastic (PanIN) lesions or primary tumors [154]. The same pattern of reprogramming was observed in pancreatic cancer patients [291] and also in other cancer types including osteosarcoma, ependymoma, and rhabdomyosarcoma [292-294]. Therefore, it is clear that distal regulatory elements play a significant role in activating metastatic programs in different cancer types. This strongly suggests that targeting enhancers can be a highly efficient approach in managing metastatic pancreatic cancer and potentially preventing metastasis from primary sites.

Interestingly, recent studies implied that BET family members play an important role in modulating gene transcription through regulation of the 3D chromatin structure [295-297]. This 
structure creates specific compartmentalization which enable enhancers to interact with and affect specific target genes $[66,298]$. Moreover, we have previously shown that BET-dependent genes are not necessarily highly enriched for BRD4, the most studied member of the BET family [99]. Instead, while the genes that were highly affected by JQ1 treatment did not have a defined pattern of occupancy for BRD4 at their respective transcription starting site (TSS), they did display a better correlation with tissue-specific BRD4-occupied enhancers. Concordantly, Cao et al. [299] reported that expression of genes can be predicted in part by the activity of their enhancers alone. While this phenomena was observed in other systems, it is highly probable that gene dependence follows the same specificity paradigms in various contexts [300]. Indeed, BET inhibitors were observed to exert marked anti-proliferative effects in metastatic melanoma via deactivating the super enhancer of the oncogenic Adhesion Molecule With Ig Like Domain 2 (AMIGO2) [301]. The BET-dependent super enhancer activating Aldehyde Dehydrogenase 2 Family (ALDH), which promotes resistance to chemotherapy and disease recurrence, was identified as a promising target in ovarian cancer [302]. In the highly metastatic Merkel cell carcinoma, BET inhibition is highly effective as it downregulates MYC by targeting its putative super enhancer [303]. Altogether, BET inhibitors are strong candidates for treating metastatic pancreatic cancer and can potentially be used as adjuvant therapies to prevent metastasis and disease recurrence if found safe in further clinical studies. These inhibitors in particular can play this unique role because, as previously discussed, reprogramming of distal regulatory elements is a hallmark of metastatic phenotype and these enhancers are frequently particularly sensitive to BET inhibition. 


\subsubsection{Effect of Bromodomain and Extraterminal Inhibition on Distal Regulatory}

\section{Elements}

Recent efforts focusing on uncovering the mechanisms by which BET inhibitors affect the proliferation of cancer cells in different systems largely confirmed the implication of distal regulatory elements in mediating the observed effects. Interestingly, a particular subtype of these elements called "super enhancers" are remarkably associated with BET members and can be partly defined by the intensity of BRD4 occupancy at these regions [123, 131]. We have previously tested the validity of the super enhancer concept which suggests that enhancers follow the rule of the vital few (the Pareto principle) where a low percentage of regulatory elements are responsible for most effects on the regulation of gene transcription [304]. While we have failed to discern such a pattern, many studies have indeed defined certain dependencies of crucial enhancers, whether "super" or "typical", on BET members.

In diffuse large B-cell lymphoma, BRD4 was observed to be more preferentially enriched at enhancers. Interestingly, BET-dependent super enhancers of master regulatory transcription factors were correlated with the anti-proliferative effects of JQ1 observed in this system [305]. Metabolic changes promoting proliferation in a specific subgroup of melanoma were reported to occur via upregulation of the BET-dependent super enhancer for Peroxisome Proliferative Activated Receptor-y (PPARG) Coactivator 1 a (PGC-1a) [306]. In castration-resistant prostate cancer, BRD4 was localized at the BET inhibitor-sensitive enhancer regions of the driver oncogene, Transmembrane Protease, Serine 2 (TMPRSS2) [307, 308]. In general, these studies imply that specific BET-dependent enhancers are activated in cancerous cells, rendering them more sensitive to BET inhibition and providing a specific therapeutic target. Intriguingly, recent methods that can detect nascent RNA such as thiol(SH)-linked alkylation for the metabolic sequencing of RNA (SLAM-seq) have shown that the effects of BET inhibition are 
dose-dependent and that high doses of BET inhibitors can lead to universal pausing of transcription while low concentrations affected specific subsets of genes in leukemia cells [309]. These hypersensitive genes were not necessarily controlled by super enhancers and correlated only in sub-clusters with other transcription factors and co-activators. This underscores the complexity of BET-orchestrated specific gene regulation, which may be associated with specific clusters of enhancers, but not exclusively explained by a "super enhancer" model.

In summary, investigating the efficacy of BET inhibitors in pancreatic cancer is highly justified given the promising anti-proliferative effects seen in different models. Additionally, recent data from pancreatic cancer and other systems identify a general pattern of BET-mediated activation of enhancers, or clusters thereof, that play a major role in driving detrimental aberrant transcriptional programs such as metastasis. However, the mechanism of action of BET inhibitors is still not fully understood and is likely highly complex and context-dependent. Many challenges exist in the investigation of the role of these inhibitors including the limitations of current techniques, measuring the anti-proliferative effects for BET inhibitors as a general readout for efficacy, disregarding the bromodomain-independent roles of BETs, and the prejudiced focus of research on only one member of the BET family, BRD4. While BRD2 and BRD3 share a high homology with BRD4, their functions and specific roles are not well-defined [211, 310]. BRD2 has garnered some attention due to recent reports that it frequently co-localizes with CCCTC-binding Factor (CTCF), an important insulator protein that demarcates transcriptional boundaries [297]. Both BRD2 and BRD3 affect gene transcription through different mechanisms than BRD4, namely by interaction with specific transcription factors such as E2F and GATA binding protein 1 (GATA1), respectively [311, 312]. In leukemia, the knockdown of either BRD2 or BRD3 failed to recapitulate the effects of BET inhibition or BRD4 manipulation, implying that they have a lesser role in this system, in contrast to the reported role in pancreatic cancer [287, 309]. Adding to the complexity is the proposed various roles of the different bromodomains 
within each member, namely BD1 and BD2 [207]. New targeting techniques utilizing proteintargeting chimeric molecules (PROTACs) can mark BET proteins for proteasomal degradation and will further shed light on the acetylation-independent effects of BETs [313, 314]. A deeper understanding of each of the BET proteins, their bromodomains, as well as extraterminal domains will enable us to use these agents in a safe and effective manner. Generally, BET inhibitors exert promising synergistic effects in pancreatic cancer with other agents, such as chemotherapeutic drugs such as gemcitabine [209] and epigenetic inhibitors such as HDAC inhibitors [315].

\subsection{Histone Deacetylase Inhibitors in Pancreatic Cancer}

Histone modifications can be active marks such as acetylation of histone 3 at lysine 27 (H3K27ac) and methylation of histone 3 at lysine 4 (H3K4me1), or repressing marks such as trimethylation of histone 3 at lysine 27 (H3K27me3) or ubiquitination of histone 2A at lysine 119 (H2Aub) [1]. Histone acetylation is a marker associated with transcriptional activation and its manipulation can be beneficial in attenuating detrimental pathways in pancreatic cancer. HDAC inhibitors which inhibit the removal of protein lysine acetylation marks have a broader effect on gene expression [316] in comparison to BET inhibitors. HDAC inhibitors are classified based on their homology to yeast into different classes (Class I-IV) [317]. These classes differ in their domains, expression and effects, and their different roles are still to be clearly defined. HDAC inhibitors differ in their potency and selectivity but mainly show promising anti-proliferative effects in cancer with the main rationale of reactivating silenced tumor suppressor genes and reversing deregulated deacetylation of histones in cancer [318, 319]. 


\subsubsection{Role and Effects of Histone Deacetylase Inhibitors in Pancreatic Cancer}

Histone deacetylase inhibitors were observed to induce p53-mediated pro-apoptotic effects in pancreatic cancer cells [320]. Additionally, they selectively inhibited proliferation of pancreatic cancer cells by affecting their aerobic metabolism and rendering them more sensitive to glycolytic inhibition [321, 322]. In general, specific HDAC inhibitors that targeted certain HDAC classes showed variable effects with pan-HDAC inhibitors causing the most marked antiproliferative effects [323]. While HDAC inhibition was found to potentiate the effects of gemcitabine in vitro and was reported to overcome its resistance, a clinical study combining HDAC inhibitors with gemcitabine in pancreatic cancer patients was prematurely terminated because the observed benefits did not outweigh the marked adverse events [194, 324-326]. HDAC inhibitors were also reported to suppress metastasis as HDACs were described to mediate the repressor action of the Zinc Finger E-Box Binding Homeobox 1 (ZEB1) on the promoter of the known epithelial marker, calcium-dependent adhesion protein-1 (CDH1) [327329]. Interestingly, we have also shown that HDAC inhibition attenuates epithelial-tomesenchymal transition (EMT) and decreases stem-like properties in pancreatic cancer cells [330]. Given that both BET and HDAC inhibition show anti-proliferative and metastasissuppressive effects in pancreatic cancer, Mazur et al. [315] combined these two agents in vitro and in vivo and observed a synergistic effect mediated by upregulation of the pro-apoptotic p57 protein. Synergy between those two agents appears at first glance to be paradoxical due to the fact that HDAC inhibitors stabilize a histone mark whose recognition is blocked by BET inhibition. However, both agents may work by attacking related transcriptional mechanisms. As previously mentioned, distal regulatory elements play a significant role in the mechanism of action of BET inhibitors. In contrast, the effects of HDAC inhibitors at these regions are less often reported. Recent studies have investigated the role of HDAC inhibitors on enhancer 
activity, which might further clarify the mechanisms by which these inhibitors act and identify new approaches to use them safely and effectively.

\subsubsection{Histone Deacetylase Inhibition Role at Distal Regulatory Elements}

As HDAC inhibitors stabilize a histone mark associated with active transcription, it is expected that it will lead mainly and directly to an upregulation of dependent genes. Surprisingly, we have detected a significant set of genes that are downregulated upon treatment with selective inhibitors of class I HDACs in the highly metastatic pancreatic cell line L3.6pl [330]. We have observed that while promoters of these downregulated genes gain acetylation as expected, individual associated distal regulatory elements of these genes show a dramatic loss of acetylation and better correlation with gene regulation. This has also been observed in the colorectal cancer cell line, HCT116, where treatment with an HDAC inhibitor also leads to the loss of H3K27ac at certain enhancer regions in a concentration-dependent manner [331]. However, this decrease is not universally observed at all enhancers as an increase of H3K27ac by HDAC inhibition at the enhancer of the pro-apoptotic B cell lymphoma-2-like 11 (BIM) gene was reported in triple negative breast cancer [332]. Consistent with a particular effect on enhancer activity, HDAC inhibition has been shown to repress enhancer RNA (eRNA) expression to a higher extent than BET inhibition in breast cancer cells [333].

Therefore, it can be concluded that HDAC inhibitors affect distal regulatory elements in a manner that is distinct from promoters, probably decreasing acetylation at a subset of specific enhancers, thereby affecting gene transcription in a more complex and diverse manner. Such regulation can be partially explained by the status of activation of the enhancer itself prior to treatment [331]. Enhancers can either be in an active state and marked by H3K27ac and H3K4me1, inactive with only H3K27me3, or poised with a lower threshold to be activated via being marked by the H3K4me1 active mark and H3K27me3 repressive mark [64]. Sanchez et 
al. [331] demonstrated that enhancers which are already active are usually inactivated by HDAC inhibition while poised enhancers show a tendency to be activated. Another very important aspect that may play a central role in defining the response of an enhancer to HDAC inhibition is the repertoire of transcription factors expressed in the cellular system and their importance for cellular phenotype and tumorigenic gene expression. It was reported that HDAC inhibitors not only exert their effects on acetylated histones, but also on acetylated transcription factors [334, 335]. Notably, acetylation of the pioneer transcription factor, Forkhead Box A1 (FOXA1) was shown to directly and negatively affect its ability to bind chromatin [336]. Remarkably, FOXA1 was recently shown to be specifically enriched in enhancer regions that are gained in metastatic pancreatic organoids [154]. Accordingly, we postulate that HDAC inhibitors can be used to attenuate the binding of FOXA1 to these enhancers leading to their deactivation. Indeed, enhancer regions that we identified as being lost following HDAC inhibitor treatment were found to be enriched for FOXA1 occupancy in another pancreatic cancer cell line [337] (Figure 14A). Interestingly, genes targeted by this mechanism were unaffected by BET inhibitor treatment [337]. Thus, combined treatment with HDAC and BET inhibitors can potentially simultaneously target different sets of activated enhancers to decrease the activation of reprogrammed enhancers synergistically and more effectively, activating aberrant transcriptional programs such as metastasis. This presents a model in which these two apparently counteracting agents can work together forming a successful therapeutic regimen in metastatic pancreatic cancer (Figure $14 \mathrm{~B}$ ). 
(a)

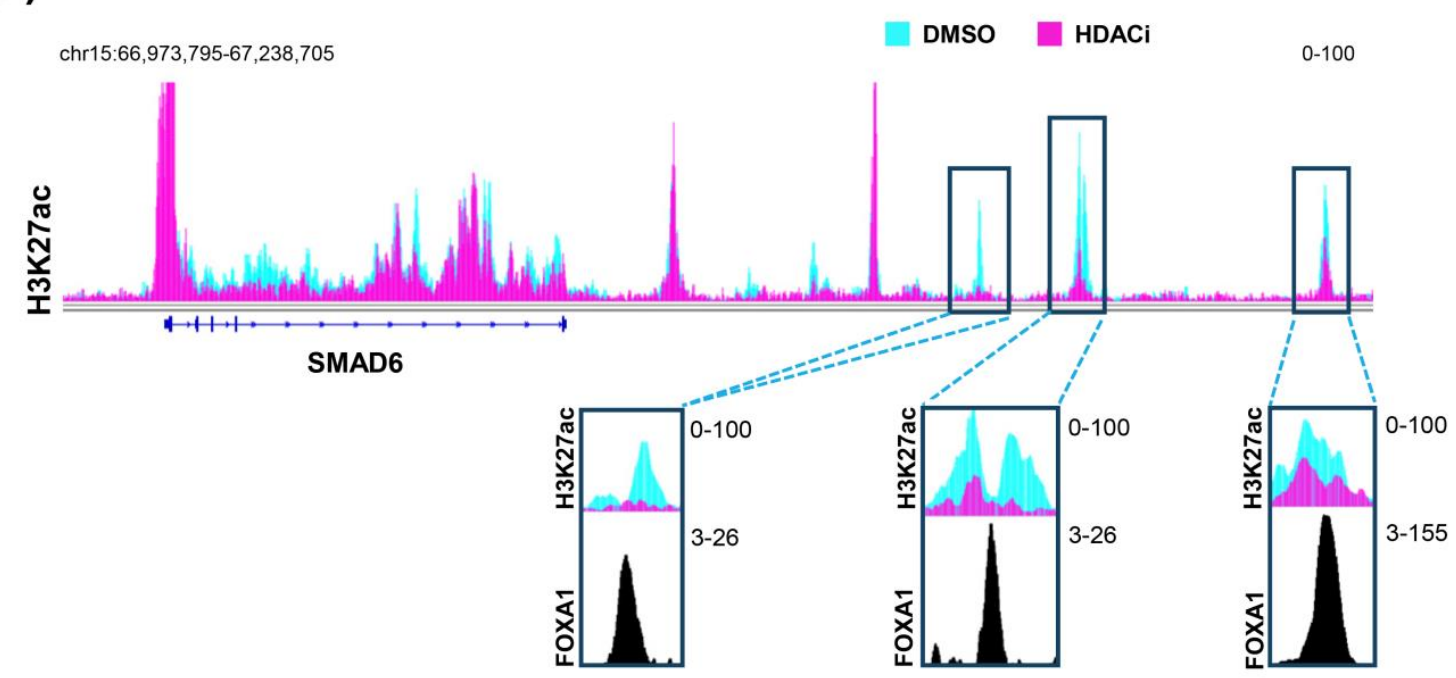

(b)

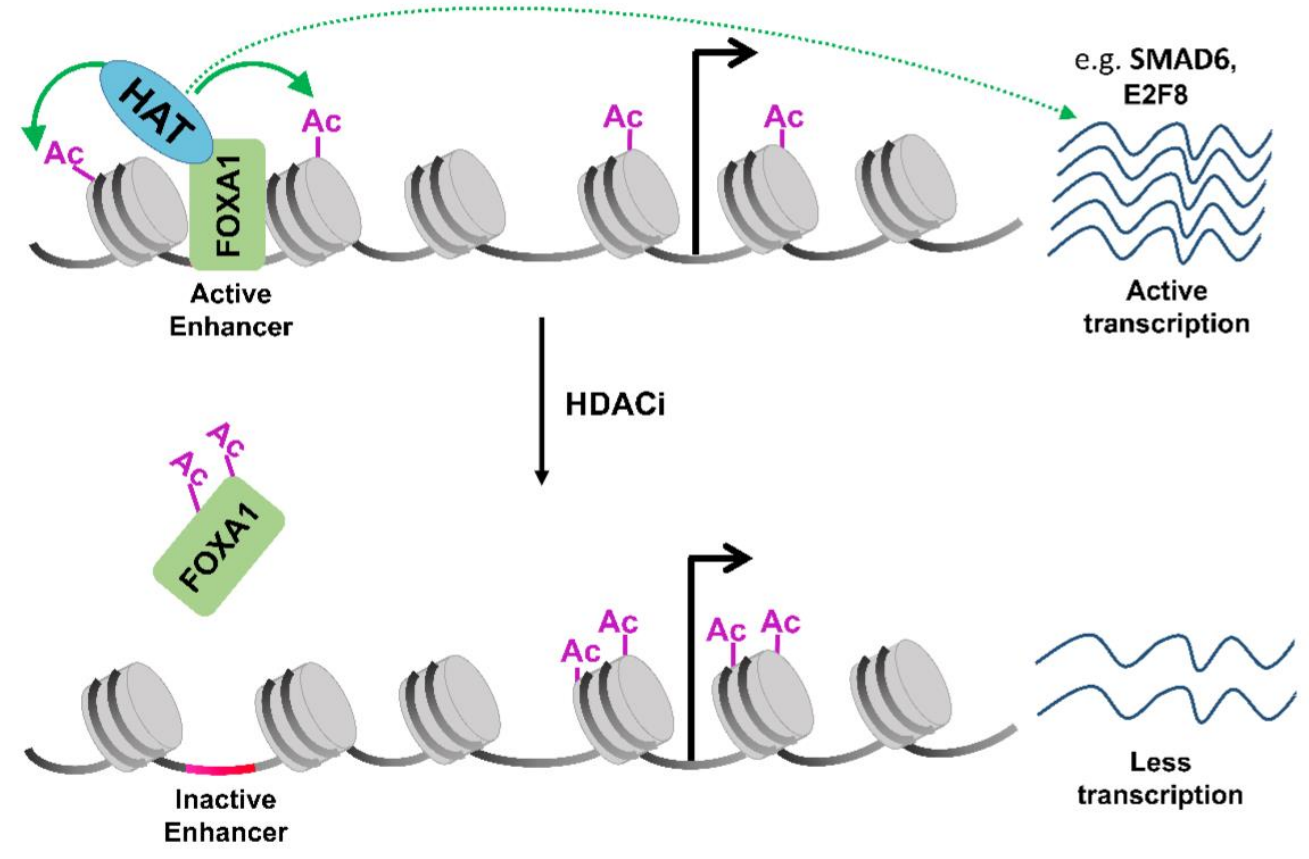

Figure 14 : HDAC inhibition and FOXA1 at distal regulatory elements. (a) H3K27ac profiles in L3.6pl cells at SMAD6, a gene that is downregulated upon treatment with class I HDAC inhibitor (HDACi) showing distal regulatory regions that dramatically lose $\mathrm{H} 3 \mathrm{~K} 27 \mathrm{ac}$ and co-localize with FOXA1 in the CFPAC1 pancreatic cancer cell line; (b) A schematic model showing an enhancer region activated by FOXA1 leading to acetylation of histones by a histone acetyltransferase (HAT), which leads to the activation of the gene. Upon treatment with $\mathrm{HDACi}$, increased FOXA1 acetylation attenuates its binding to chromatin leading to downregulation of the gene. DMSO: Dimethylsulfoxide; HDAC: histone deacetylase; FOXA1: Forkhead Box A1. (See Appendix A for details of data analysis) 


\subsection{Enhancers as an Optimal Paradigm for Therapeutic Targeting of} Pancreatic Cancer

Based on the evidence discussed above, it is clear that distal regulatory elements play a special role in the scope of the effects of BET and HDAC inhibitors. Thus, it is highly probable that these elements will emerge as a major target of therapy in multiple diseases in the upcoming years. Many positive attributes contribute to the adequacy of enhancers as a target for therapy and manipulation. To activate a certain gene, the transcriptional machinery has to be recruited by transcription factors to the promoters of these genes [338]. Diversity in transcription factor recruitment and abundance are thus very important in regulating gene activation in different contexts and systems. Distal regulatory elements provide a platform with vast variability and substantial magnitude for recruitment of various transcription factors, thereby enabling regulation of gene transcription in a temporal and spatial manner. This means that in certain systems, driver oncogenic pathways can be activated by different transcription factors and enhancers, thus creating a dependence which can be specifically targeted. For example, different enhancers drive the activation of $M Y C$ in various systems. In colorectal cancer, the long non-coding RNA, Colon Cancer Associated Transcript 1 (CCAT1) is highly active and plays a significant role in $M Y C$ activation [251, 339]. Consistent with the importance of the Wnt signaling pathway in colorectal cancer, we observed that this enhancer is highly occupied by Wnt-responsive $\beta$-catenin-dependent transcription factor TCF7L2 (Figure 15) [339]. This implies that Wnt-signaling mediated activation of $M Y C$ in colorectal cancer utilizes a specific mechanisms of activation which can be potentially be targeted by HDAC inhibitors, as they were reported to deplete TCF7L2 [340]. Interestingly, CCAT1 was also reported to be play a tumorigenic role in pancreatic cancer [341]. In other systems such as prostate cancer, MYC is activated by a different enhancer called Prostate Cancer Associated Transcript 1 (PCAT1). Consistent, with the androgen receptor (AR) being a primary driver of prostate cancer, we 
observed this enhancer to be particularly occupied by AR in LNCaP prostate cancer cells [251, 342-344]. Alternatively, the PVT1 oncogene is active in many other systems such as leukemia and plays a similar role as an enhancer of the $M Y C$ gene [345]. Analogous to the tumor- and context-specific effects shown for TCF7L2 and AR in colorectal and prostate cancers, respectively, we observed an enrichment of the hematopoietic transcription factor GATA-2 on the MYC enhancer within the PVT1 gene [339]. Together, these examples show the complexity by which diverse distal regulatory elements utilize specific transcription factor repertoires to induce common oncogenic pathways. We postulate that distinct, but similar transcription factor networks will also be discovered in pancreatic cancer which can be specifically targeted by inhibitors of BET, HDAC or other epigenetic regulators. Importantly, activation of oncogenes such as $M Y C$ by alternative BET-independent transcriptional pathways can lead to resistance to BET inhibitors in leukemia cells [346]. Thus, identifying which enhancers are specifically active in pancreatic cancer cells and identifying and targeting their dependencies will play an important role in the optimal application of BET inhibitors in the treatment of pancreatic cancer. Furthermore, targeting tumor-specific enhancer regions will be more likely to spare normal cells and may possibly lead to less long term adverse effects [347]. Development of highly specific BET inhibitors supports this theory by showing selective anti-proliferative effects in certain cancer cell lines while not affecting other systems [348]. Future studies will test the validity of this rationale upon successful prolongation of patient survival. 


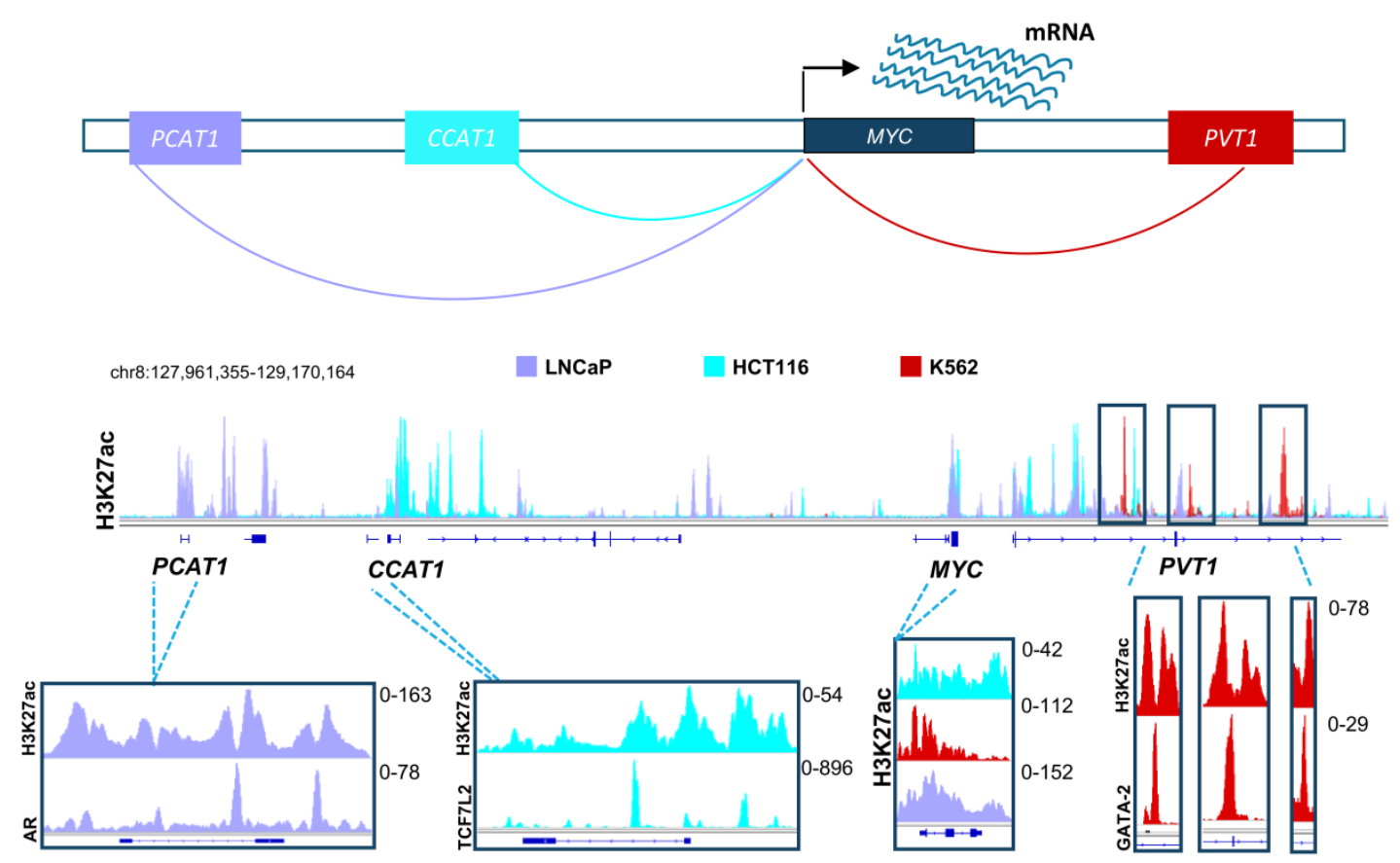

Figure 15 : A schematic model showing distal regulatory mechanisms controlling the expression of the MYC gene in different systems, namely in prostate cancer (shown in light purple), colorectal cancer (shown in turquoise), and leukemic cell lines (shown in red). The H3K27ac layered profile in these different cell lines uncover differential activation of unique enhancers in each system. These enhancers are specifically enriched with driver transcription factors (androgen receptor, AR; TCF7L2 and GATA2), which are hallmarks of these tumor types. TCF7L2: Transcription Factor 7-like 2; GATA2: GATA binding protein-2. (See Appendix A for details of data analysis)

\subsection{Targeting Transcription Factors in Pancreatic Cancer: A Code for}

\section{Specificity}

Enhancers are known to be highly-bound by specific transcription factors that mediate transcriptional activation of target gene expression through the recruitment of other activators and transcription initiators [349]. When transcription factors co-localize with a factor of interest, BETs for example, these factors will naturally play a significant role in its mechanism and effects. Interestingly, the BET context-specific effects discerned in the vast body of literature available can probably be explained by the different interacting factors at specific sites where 
BET members co-localize with other transcription factors. BET-dependent enhancers require BET members and certain transcription factors for their activation, while other BET-independent enhancers might have other factors and activation pathways which make them tolerant to the loss of one of many activators upon BET inhibition. Furthermore, the different expression levels of these transcription factors in different systems, whether absent or highly or lowly expressed, in addition to their pioneering potential can also play a role in enhancer dependency and activity.

Paradoxically, super enhancers are by definition normally highly enriched for transcription factor binding, which can theoretically render them less dependent on one particular factor [248]. However, this is not usually the case given that, as previously discussed, BET inhibition has the ability to turn off certain BET-dependent super enhancers but deletion of individual components of "super enhancers" does not equally impair the activation of the target gene [251, 305, 306, $350,351]$. Thus, it appears that BET proteins serve to integrate the activity of transcription factors at BET-dependent enhancers in a manner such that the sum of the activity of the transcription factor binding is greater than that of the components. Subsequently, once a better understanding of transcription factor function at enhancers and promoters and the dependence of each on BET proteins has been achieved, targeting specific transcription factors or their upstream signaling pathways, possibly alone or in combination with BET inhibitors, can provide us with a new layer of specific gene transcriptional manipulation.

However, therapeutically targeting transcription factors can be quite challenging. One approach can be targeting signaling pathways that control the activity of the transcription factor of interest. For example, the transcription factor Endothelial PAS Domain Protein 1 (EPAS1 or HIF2A) was demonstrated to play a role in promoting pancreatic cancer in cells and mice [352]. A crosstalk with Wnt-signaling was identified in this system which uncovers a new target that can potentially be inhibited. Furthermore, Transforming Growth Factor- $\beta$ (TGF $\beta$ ) signaling was reported to 
cooperate with mutant p53 to mediate distal metastasis in pancreatic cancer mouse models [353]. The activation of Nuclear Factor kappa-light-chain-enhancer of activated B cells (NF-kB), which also promotes EMT in pancreatic cancer, can also be inhibited by blocking its activation via NFKB inhibitor- $\alpha$ (IKBa) phosphorylation [354]. Another approach to target transcription factors is to attenuate their recruitment by affecting their ability to bind chromatin as previously mentioned for FOXA1 and its acetylation. This can also be achieved by designing specific inhibitors that prevent the binding of DNA to a certain transcription factor [355]; however this approach has proven to be very difficult for therapeutic application. Another approach can be targeting cooperating transcription factors.

For example, NF-kB cooperates with E26 transformation-specific (ETS) transcription factors to recruit BET members to activate genes, rendering these sensitive to BET inhibition [356, 357]. In general, transcription factors can play specific roles in a context-dependent manner based on the combinatorial repertoire of transcription factors expressed, thereby enabling a given transcription factor to activate a different set of genes and programs, dependent upon the expression of other factors. Therefore, identifying important transcription factors playing a role in aberrant transcriptional activation may uncover specific targets that can be manipulated by available inhibitors.

\subsection{Conclusions: Unconventional Epigenetic Agents Should be Used in Unconventional Ways}

Epigenetic agents are a special subclass of drugs whose targets and effects are dependent on the epigenetic and transcriptional landscape of each system. In general, a major trend is seen where low concentrations of these agents affect hypersensitive dependent genes and higher concentrations frequently display a more universal effect [309] . So far, gene transcription modulatory agents have been used to initiate cell cycle arrest and/or apoptosis and mainly 
administered at high levels just under their maximum tolerable dose (MTD). We speculate that administering these agents at these doses likely influences their specificity and probably promotes many of the intolerable adverse effects that might lead to premature termination of clinical studies. In fact, higher doses of such inhibitors, which lead to cell cycle arrest, may in fact impede the activity of many chemotherapeutic agents, while lower concentrations which elicit specific transcriptional reprogramming may have minimal effects on their own, but significantly synergize with other therapies. To ensure the maximum utilization of these agents, concentrations lower than MTD should be taken into consideration when performing pharmacodynamic and pharmacokinetic studies in vivo. However, these studies will face major challenges as markers that can be detected ex vivo to measure efficacy are still not clearly defined. Ideally, a marker should be easily detectable and highly sensitive, for example enhancer RNA of BET-dependent genes or stable messenger RNA of a highly dependent and hypersensitive gene. Using profoundly regulated gene levels in blood as a marker for BET inhibition efficacy uncovered that BET inhibitors exert their effects at lower doses than MTD in progressive lymphoma patients [358].

In this review, we summarized data pertaining to the effects of BET and HDAC inhibitors, two of the most promising epigenetic agents, in pancreatic cancer. We draw the conclusion that these agents likely mediate their specific effects through distal regulatory elements. By investigating the potential utility of these agents in lower concentrations, we may be able to uncover their potential as safe adjuvant therapies in combination with other standard of care treatments to manage and prevent recurrence of pancreatic cancer and various malignancies in general. 


\subsection{Methods/Appendix A: Parameters for data analysis used in figure generation}

Chromatin immune-precipitation profiles which are shown in Figures 1 and 2 as examples were downloaded from the Encyclopedia of DNA Elements (ENCODE) consortium when available (H3K27ac in HCT116: GSM2534277; TCF7L2 in HCT116: GSM782123; H3K27ac in K562: GSM733656; GATA-2 in K562: GSM935373) [339]. Other profiles were downloaded from the European Nucleotide Archive (H3K27ac in LNCaP: SRR2566837 [342]; AR in LNCaP: SRR4025870 [343]; H3K27ac in L3.6pl: SRR5042516,18-21 [330]; FOXA1 in CFPAC1: SRR1736462 [337]). Reads were mapped to the hg19 genome using BOWTIE/2.2.5 [359] and converted to bam using SAMTOOLS/1.4 [360]. DEEPTOOLS/2.4.0 [361] was used to produce bigwig files with ignoring the duplicates and extending the reads for 200 base pairs. Bigwig files were viewed using IGV $2.4[362,363]$.

\subsection{Author Contributions}

F.H.H. and S.A.J. have conceived the ideas for this work and wrote the manuscript.

\subsection{Acknowledgements}

F.H.H. was funded by the German Academic Exchange Service (DAAD). This work was funded by grants from the Deutsche Krebshilfe (PiPAC Consortium) [70112505], Deutsche Forschungsgemeinschaft (DFG) [JO 815/3-2], and the Roggenbuck Foundation to S.A.J.

\subsection{Conflicts of Interest}

The authors declare no conflict of interest. 


\section{Manuscript I}

\section{CHAPTER 4}

\section{DeltaNp63-dependent super enhancers define molecular identity in pancreatic cancer by an interconnected transcription factor network}

Short Title: DeltaNp63 define molecular subtypes of cancer

Feda H. Hamdan ${ }^{1}$ and Steven A. Johnsen ${ }^{1, *}$

${ }^{1}$ Section of Tumor Epigenetics, Department of General, Visceral and Pediatric Surgery, University Medical Center Göttingen, 37075 Göttingen, Germany

* To whom correspondence should be addressed. Tel: +49 55139 13711; Fax: +49 55139 12297; Email: steven.johnsen@med.uni-goettingen.de

\section{Keywords}

Super enhancers, transcription factors, DeltaNp63, HIF1A, squamous, basal, FAT2, BHLHE40, molecular subtypes

This manuscript has been peer-reviewed and is currently under revision for PNAS Journal,

Contributions: All Figures and analysis was done by F.H.H. F.H.H. and S.A.J. conceived the ideas for this project and wrote the manuscript. 


\subsection{Abstract}

Molecular subtyping of cancer offers tremendous promise for the optimization of a precision oncology approach to anti-cancer therapy. Recent advances in pancreatic cancer research uncovered various molecular subtypes with tumors expressing a squamous/basal-like gene expression signature displaying a worse prognosis. Through unbiased epigenome mapping, we identified deltaNp63 as a major driver of a gene signature in pancreatic cancer cell lines, which we report to faithfully represent the highly aggressive pancreatic squamous subtype observed in vivo, and display the specific epigenetic marking of genes associated with decreased-survival. Importantly, depletion of deltaNp63 in these systems significantly decreased cell proliferation and gene expression patterns associated with a squamous subtype and transcriptionally mimicked a subtype switch. Using genomic localization data of deltaNp63 in pancreatic cancer cell lines coupled with epigenome mapping data from patient-derived xenografts, we uncovered that deltaNp63 mainly exerts its effects by activating subtype-specific super enhancers. Furthermore, we identified a group of 45 super enhancers that are associated with poorer prognosis and are highly dependent on deltaNp63. Genes associated with these enhancers included a network of transcription factors, including HIF1A, BHLHE40, and RXRA, which form a highly intertwined transcriptional regulatory network with deltaNp63 to further activate downstream genes associated with poor survival.

\subsection{Significance statement}

Distinct molecular subtypes of pancreatic cancer have recently been identified with the squamous subtype exhibiting a particularly poorer prognosis. Precision-medicine approaches are needed in pancreatic cancer due to its very poor prognosis. While deltaNp63 was identified as a hallmark of the squamous phenotype in other cancers, mechanisms by which it defines this 
signature were largely unknown. This study uncovers deltaNp63 as a major driver of the squamous subtype by activating a subgroup of distal regulatory elements termed super enhancers. This leads to the activation of a network of transcription factors propagating the activation signal to numerous pathways. Importantly, an epigenetic signature identified in this study is capable of accurately identifying squamous subtype samples in pancreatic cancer patient-derived xenograft tumors.

\subsection{Introduction}

Distinct molecular subtypes in cancer are defined by different deregulated pathways, mutational profiles, and aberrant transcriptional programs, and may potentially be leveraged to optimize therapy and elucidate mechanisms in a disease that is characterized by a particularly high degree of heterogeneity [364]. Molecular stratification of breast and colorectal cancer, for example, revolutionized therapy for these malignancies and extended our knowledge about the pathways and mechanisms involved in disease development and progression [365-367]. Recently, analyses in pancreatic cancer, which has a consistently low survival rate, uncovered various molecular subtypes with different characteristics and prognoses [153, 213-215, 337, 368].

Collisson et al. used human and mouse samples in addition to pancreatic cancer cell lines to identify homogenous patterns of gene expression and identified three subtypes, referred to as classical, exocrine-like, and quasi-mesenchymal, with the latter being particularly correlated with poor prognosis [213]. Further optimization of molecular stratification by filtering stromal profiles further grouped the molecular subtypes of pancreatic cancer into classical-like and basal-like with the latter corresponding to the worse prognosis seen in the quasi-mesenchymal phenotype [214]. Extending these analyses to include mutational backgrounds of patients and DNA 
methylation states in addition to gene expression revealed 4 subtypes including the highly aggressive squamous subgroup [215]. Further analysis confirmed the identification of specific patterns of expression with one molecular subtype, irrespective of nomenclature, representing a small subgroup of pancreatic cancer patients with a particularly poor prognosis [337, 368].

While more advances are being made in the analytical aspect of subtyping pancreatic cancer, the molecular mechanisms underlying these gene signatures are still largely unclear. Bailey et al. identified deltaNp63 activity as a hallmark of the squamous subtype, which overlaps with its basal-like counterpart and profoundly correlates with worse prognosis [215, 368]. p63 is a transcription factor of the p53 family which has two major isoforms including the transactivation domain-containing isoform, TAp63, and the shorter isoform, deltaNp63 [227]. DeltaNp63 was reported to play a crucial role in keratinocyte differentiation and its expression was shown to be a defining feature of basal cells and squamous cell carcinomas [230, 369, 370]. Concordantly, deltaNp63 is expressed in many squamous-like cancers such as esophageal squamous cell carcinoma [233, 371], head and neck squamous carcinoma [372], and lung squamous cell carcinoma [373, 374]. p63 was found to drive epidermal differentiation through distal regulatory elements associated with its target genes [375]. However, to date a role for deltaNp63 in pancreatic cancer has remained largely unclear.

An early report described an upregulation of deltaNp63 in a group of pancreatic cancer cells displaying a squamous-like phenotype, while normal pancreata were completely devoid of deltaNp63 expression [239]. Interestingly, depletion of the histone demethylase KDM6A led to the activation of super enhancers regulating deltaNp63 and enriched for a more squamous-like phenotype in pancreatic cancer cells [212]. Super enhancers are clusters of distal regulatory elements which are highly enriched for transcription factor binding and have a high potential to affect target genes [123, 124, 248]. Our previous work identified tissue-specific patterns of gene 
expression which were particularly coupled to transcription factor and co-factor recruitment to distal enhancer regions rather than occupancy in the proximal promoter region [99, 330, 376].

In this study, we performed an unbiased analysis of epigenomic gene activation profiles and identified deltaNp63 as a major driver of gene activation in a particularly lethal subtype of pancreatic cancer. We report that L3.6pl and BxPC-3 pancreatic cancer cell lines represent appropriate cell culture models of the squamous molecular subtype described in patients. Here, we uncovered a major dependence of subtype-specific super enhancers on deltaNp63. Furthermore, we further confirmed the analyses of our model cell lines and significantly expanded the relevance of the findings by comparing our results with data from patient-derived xenograft samples. Using this approach we identified 45 super enhancers that signify the squamous subgroup and are associated with genes that are highly deltaNp63-dependent and correlate with poor prognosis in pancreatic cancer. Among these genes, we uncovered a highly interactive transcriptional regulatory hub including deltaNp63, HIF1A, RXRA, and BHLHE40, where these factors activate one another as well as downstream genes. Altogether, our study elucidates underlying mechanisms by which deltaNp63 drives gene expression patterns associated with the squamous molecular subtype in pancreatic cancer and identify a number of super enhancers that may potentially be used to identify this subgroup in order to stratify patients with poorer prognosis in a simple and accessible manner. 


\subsection{Results}

\subsubsection{DeltaNp63 is a major driver of differential gene activation in specific pancreatic cancer cell lines and patient-derived xenografts}

In order to elucidate the differences in the epigenomic landscape of commonly-studied pancreatic cell lines, we performed chromatin immunoprecipitation followed by high throughput sequencing (ChIP-seq) for histone acetylation at lysine 27 of histone 3 (H3K27ac) in Panc-1, BxPC-3 and the highly metastatic L3.6pl cell lines. As H3K27ac marks active regions, including promoters and enhancers, it gives a comprehensive insight into active gene transcription. Hierarchical clustering of H3K27ac peaks in all cell lines identified three clusters with the first and second having low and high signal, respectively, in all cell lines. Only the third cluster showed marked enrichment in BxPC-3 and L3.6pl compared to low enrichment in Panc-1 (Figure 16A). GREAT analysis for the 15,286 regions included in cluster 3 (out of 88,773 total regions) showed validated targets of deltaNp63 as the most significant hit for the genes associated with these regions (Figure 16B). To further investigate regions that are specifically marked in L3.6pl and BxPC-3, we performed unbiased differential binding analysis for H3K27ac in the three cell lines and identified 9,348 regions that are significantly gained in BxPC-3 and L3.6pl and have at least a 5-fold enrichment compared to Panc-1, henceforth referred to as H3K27ac gained regions (Figure 16C). Consistent with initial findings based on hierarchical clustering, differential occupancy analyses revealed targets of deltaNp63 to be the most highly significant pathway enriched for genes associated with H3K27ac gained regions (Figure 16D). To determine the extent to which deltaNp63 may play a direct role in determining the differential marking of H3K27ac gained regions, we performed ChIP-seq for p63 in L3.6pl and BxPC-3 and found that approximately one-third of the H3K27ac gained regions are occupied by p63 (Figure 
16E). Thus, these unbiased analyses provide evidence suggesting that p63 is a major driver of genes specifically activated in L3.6pl and BxPC-3 cell lines compared to Panc1 cells.

To examine if the epigenome patterns observed in L3.6pl and BxPC-3 also occur in patient tumors and not exclusively in vitro, we examined H3K27ac profiles from 24 pancreatic cancer patient-derived xenografts [220]. Principal component analysis using the H3K27ac gained regions identified three of the 24 xenografts as forming a distinct cluster (Figure 16F). Interestingly, these three xenografts were highly marked by H3K27ac near the transcriptional start site (TSS) of deltaNp63 compared to the rest of the samples (with three other representative samples shown as an example; Figure 16G). Analysis of RNA-seq data confirmed the expression of p63 in these tumor samples. Isoform-specific expression was confirmed by H3K27ac occupancy at the TSS of deltaNp63, but not TAp63, with only one highly p63-expressing xenograft appearing to co-express both isoforms (Supplementary Figure S1A and S1B). Importantly, H3K27ac gained regions identified in L3.6pl and BxPC3 also displayed a clear increased enrichment of H3K27ac in PDX samples expressing deltaNp63 compared to the non-expressing ones (Figure 16H). Thus, these data support that deltaNp63-driven epigenetic patterns observed in L3.6pl and BxPC-3 can also be found in pancreatic cancer patient-derived xenografts (PDX). 
A

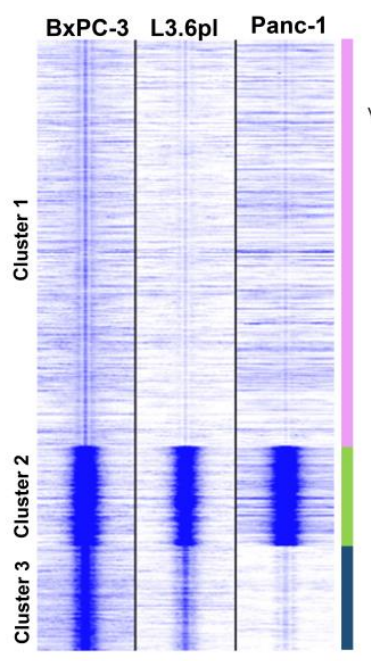

B Cluster 3

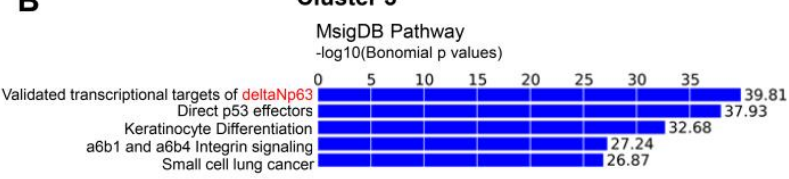

MsigDB Pathwa a6b1 and a6b4 Integrin signalin
Small cell lung cancer

C

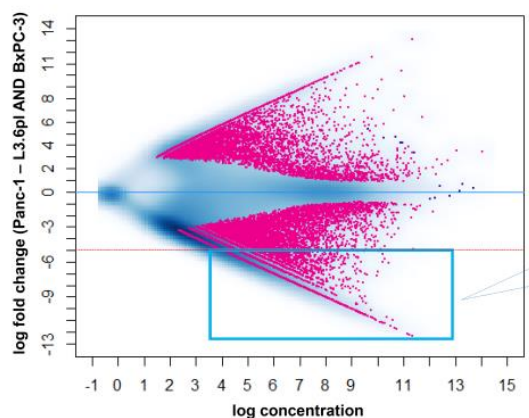

E
D Gained regions in BxPC-3 and L3.6 (Fold > 5, FDR $<0.05)$ MsigDB Pathway $-\log 10$ (Bonomial p values)
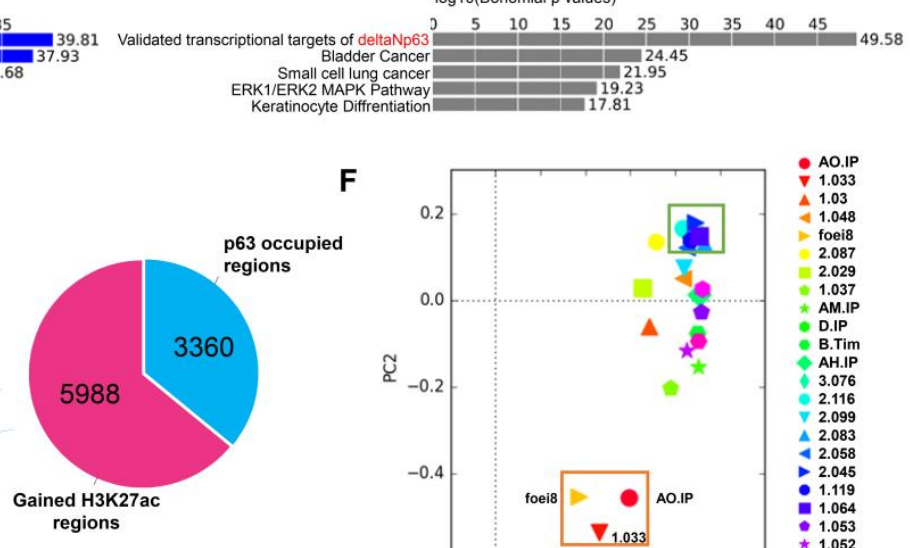

\section{$\mathbf{F}$}

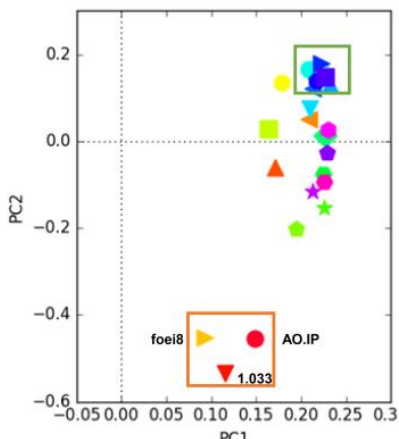

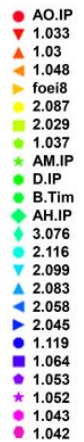

G
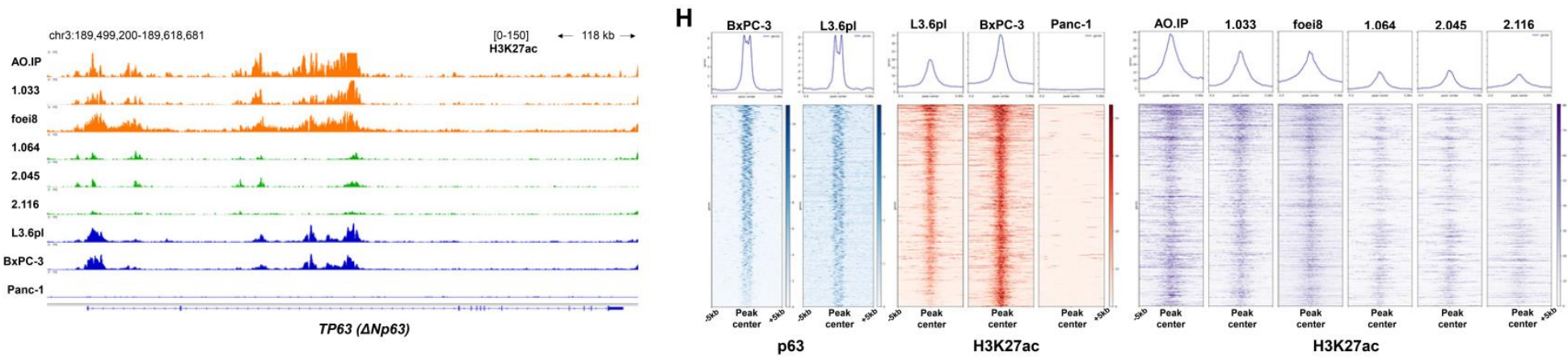

Figure 16 : DeltaNp63 drives gene activation in L3.6pl and BxPC-3. (A) Heatmap showing the enrichment of H3K27ac at the concatenated and merged peaks for BxPC-3, L3.6pl, and Panc-1. Peaks were clustered into 3 groups based on KMeans hierarchical clustering normalized to linear enrichment. Cluster 3 shows more enrichment in L3.6pl and BxPC-3 compared to Panc-1. (B) Associated genes with regions in cluster 3 defined by GREAT analysis using the default basal plus extension association rule and showing validated targets of deltaNp63 as the top hit. (C) Binding affinity plot for H3K27ac peaks in Panc-1 compared to L3.6pl and BxPC-3 showing the regions with a significant enrichment difference in pink. The blue box highlights the regions that are significantly enriched in L3.6I and BxPC-3 by more than 5 fold change, henceforth called H3K27ac gained regions (9348 regions). (D) Associated genes with the H3K27ac gained regions also showing validated targets of deltaNp63 as the top hit. (E) Pie chart depicting the percentage of the 9348 regions that are occupied by p63. (F) Principle component analysis plot for the H3K27ac profiles of 24 patient-derived xenografts named on the right limited to the 9348 regions in 1D. Orange box indicates three xenografts which clustered independently from the other samples and the green box highlights samples clustering in the opposite direction. (G) Occupancy profiles of H3K27ac at the TSS of deltaNp63 for the six xenografts highlighted in orange and green box in 1F, L3.6pl, BxPC-3, and Panc-1. Results show an enrichment for the three uniquely clustering xenografts, L3.6pl and BxPC-3, while the other samples have very low occupancy of H3K27ac. (H) Average binding profiles and heatmaps depicting the p63 and H3K27ac occupancy at the H3K27ac gained regions in L3.6pl, BxPC-3 for p63 and L3.6pl and BxPC3, Panc-1 and the six differentially clustered patient-derived xenografts for H3K27ac. 


\subsubsection{L3.6pl and BxPC-3 highly express deltaNp63 and are representative models for the squamous subtype of pancreatic cancer}

Various molecular subtypes were recently identified in pancreatic cancer based on unique gene expression profiles and distinct genomic and epigenomic signatures (Figure 17A). One of the most aggressive subgroups of pancreatic cancer identified is the squamous subtype, which is associated with worse prognosis and high expression of deltaNp63. Indeed, patient survival data from the Cancer Genome Atlas (TCGA) data confirmed that patients expressing higher p63 had a poorer prognosis than patients with lower levels (Figure 17B). As deltaNp63 was identified in our analyses to be highly correlated with L3.6pl- and BxPC-3-specific cis-regulatory regions, we next examined whether these cell lines may, indeed, represent the squamous subtype and serve as model systems for studying the molecular mechanisms driving this particularly aggressive tumor phenotype observed in vivo. Therefore, we evaluated the expression of p63 in different pancreatic cancer cell lines in the Morpheus database and verified these findings at the protein level (Figure 17C, 17D) [377]. Notably, only BxPC-3 and L3.6pl displayed high expression of deltaNp63, with DanG showing a moderate expression, and MIA Paca-2 cells specifically expressing only the TAp63 isoform (Figure $1 \mathrm{D}$ ). To further examine whether L3.6pl and BxPC-3 faithfully represent the squamous subtype, we examined whether they expressed a squamous gene signature compared to Panc-1 and, indeed, this signature was significantly enriched in both L3.6pl and BxPC-3 cell lines compared to Panc-1 cells (Figure 17E, 17G). Tendencies of enrichment for these genes were also observed in the three xenografts we identified as highly expressing of deltaNp63 (Supplementary Figure S1D). Notably, we also found that genes associated with an unfavorable prognosis in cancer patients were specifically enriched in the L3.6pl and BxPC-3 cell lines, providing further support that these cell lines may serve as a model for understanding the molecular mechanisms driving the 
Manuscript I | Feda Hamdan

aggressive characteristics of squamous-like pancreatic tumors (Figure 17F and Supplemental

Figure S1C).

A
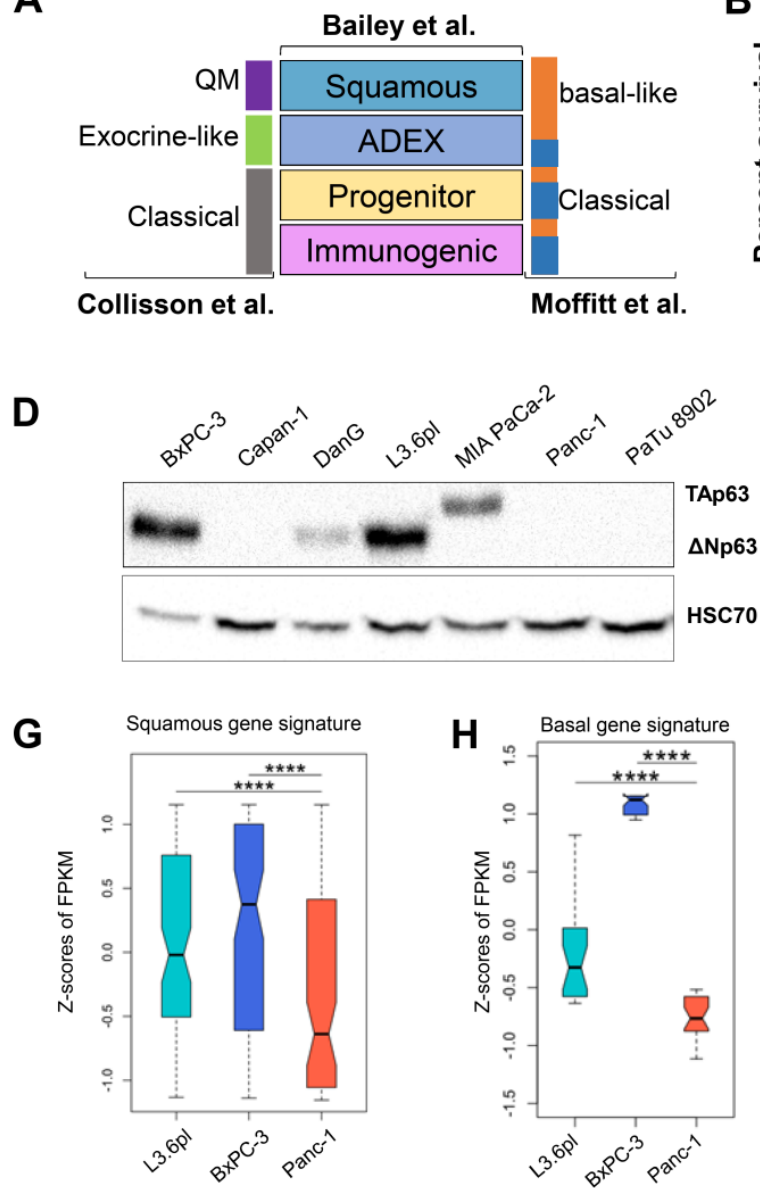

B

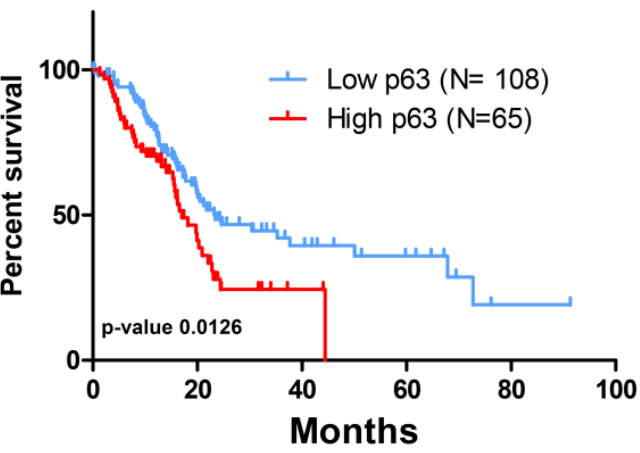

$\mathbf{E}$

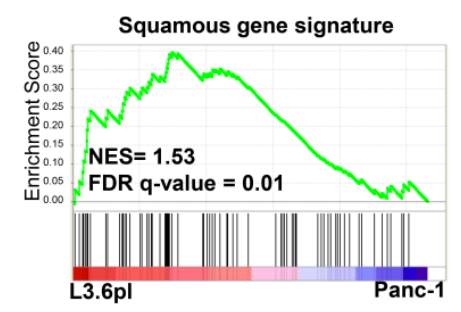

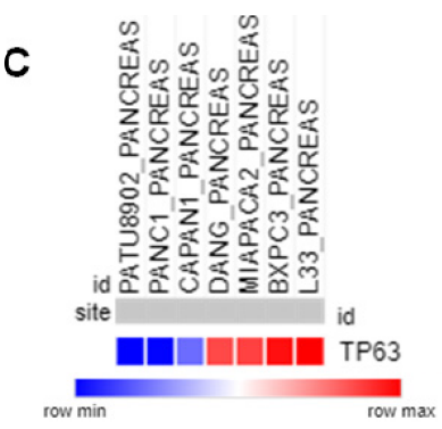

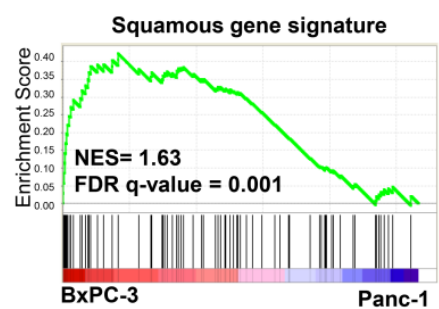

$\mathbf{F}$
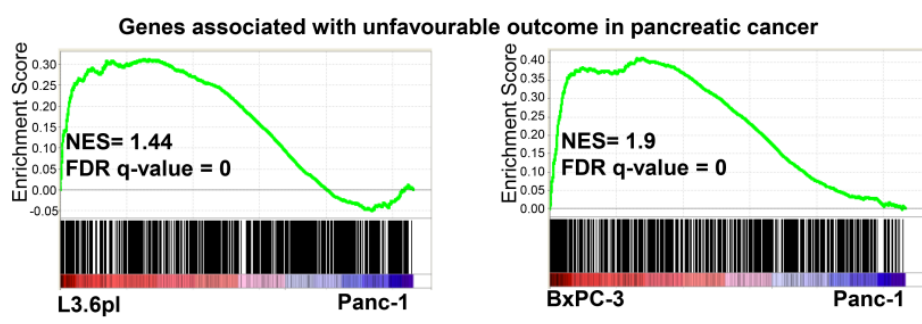

Figure 17 : L3.6pl and BxPC-3 represent the squamous subtype. (A) Graphical representation of the molecular subtypes recently defined in pancreatic cancer. (B) Kaplan-Meier plot showing the percent survival in pancreatic cancer patients (TCGA database) expressing high and low levels of p63 and showing significantly worse survival rates in patients with high levels of p63. (C) Heatmap depicting the general expression patterns of p63 in different pancreatic cancer cell lines (Morpheus database). (D) Western blot analysis of the levels of p63 in various pancreatic cell lines with HSC70 as a loading control. (E-F) GSEA plots comparing the enrichment of the squamous gene signature (E) and genes associated with unfavorable outcome in pancreatic cancer (F) in L3.6pl and BxPC-3 compared to Panc-1 using the FPKM values of all expressed genes with the NES (normalized enrichment score) and FDR (false-discovery rate) indicated on the graph. (G) Box plot showing the Z-scores of FPKM values of the squamous gene signature in L3.6pl, BXPC-3, and Panc-1. $\mathrm{n}=3$. * $\mathrm{P}$-value $<=0.05$, ${ }^{* *} \mathrm{P}$-value $<=0.01$, ${ }^{* *} \mathrm{P}$-value $<=0.001$, ${ }^{* * * *} \mathrm{P}$-value $<=0.0001$. $(\mathrm{H})$ Box plot showing the Z-scores of FPKM values of the basal gene signature in L3.6pl, BXPC-3, and Panc-1. $n=3$. ${ }^{*} \mathrm{P}$-value $<=0.05,{ }^{* *} \mathrm{P}$-value $<=0.01,{ }^{* * *}$ P-value $<=0.001,{ }^{* * *}$ P-value $<=0.0001$. 
Given that the squamous subgroup reported by Bailey et al. roughly corresponds to the basal phenotype identified by Moffitt et al., we also tested whether the basal gene signature was also enriched in L3.6pl and BxPC-3 and, indeed, observed a significant enrichment of the expression of these genes compared to Panc-1. Together, these findings confirm that L3.6pl and BxPC-3 are representative in vitro model systems of squamous/basal-like pancreatic cancer cells.

\subsubsection{Depletion of deltaNp63 alters the molecular identity of squamous pancreatic cancer cells}

To investigate the role of deltaNp63 in gene activation in L3.6pl and BxPC-3, we depleted deltaNp63 by siRNA-mediated knockdown and validated its downregulation at the mRNA and protein levels (Figure 18A, 18B). Interestingly, knockdown of deltaNp63 led to a marked decrease in proliferation in both L3.6pl and BxPC-3 cells (Figure 18C, 18D). Moreover, sphere formation in both cell lines was significantly impaired upon p63 knockdown, with the few remaining spheres that were formed displaying a more diffuse and less defined structure, particularly in the case of L3.6pl (Figure 18E, 18F). This confirmed a crucial role of deltaNp63 in driving the more aggressive phenotype in both of these cell lines. To further understand how deltaNp63 drives this phenotype, we performed RNA-seq in both L3.6pl and BxPC-3 cell lines upon knockdown of deltaNp63. Remarkably, deltaNp63 downregulation led to the reversal of the enrichment of the squamous gene signature, validating a clear and central role of deltaNp63 in driving the activation of these genes (Figure 18G). Furthermore, GSEA enrichment analysis identified MYC and HIF1A as target pathways of deltaNp63 (Figure 18H, Supplementary Table1). Notably, deltaNp63 depletion mimicked a switch from a mesenchymal to luminal phenotype defined in breast cancer. Moreover, an enrichment of pathways with decreased tumorigenesis was observed in cells with less deltaNp63 (Figure 18I, Supplementary Table 2). 
A

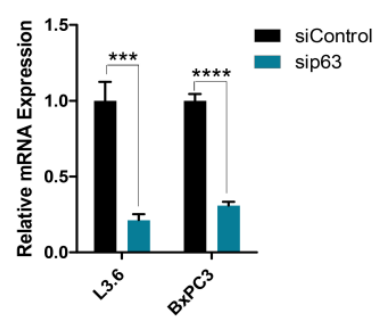

E

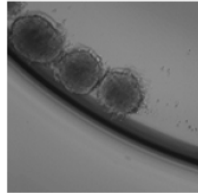

siControl

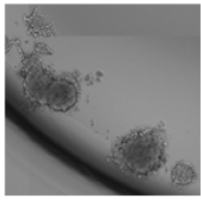

$\operatorname{sip} 63$
B

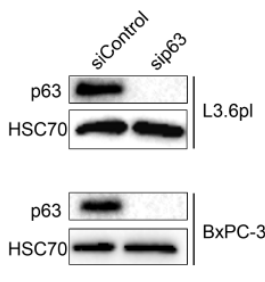

$\mathbf{F}$

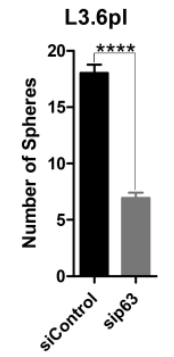

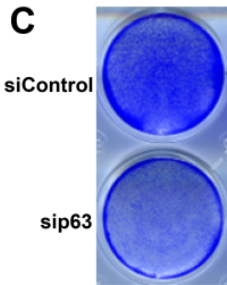

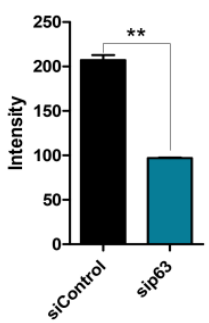

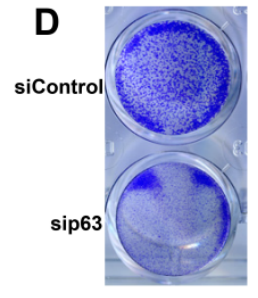

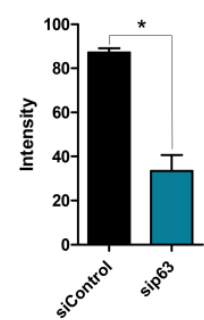

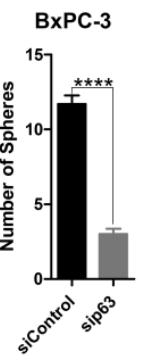

G
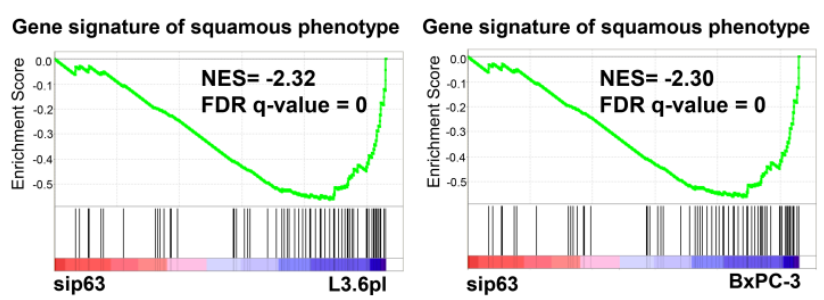

H

GSEA Enrichement in siControl L3.6 cells compared to sip63

\begin{tabular}{lcccc}
\hline NAME & SIZE & ES & NES & FDR q-Val \\
\hline SCHUHMACHER_MYC_TARGETS_UP & 78 & -0.75943 & -3.17847 & 0 \\
MANAL_HYPOXIA_DN & 283 & -0.62011 & -3.11342 & 0 \\
WONG_EMBRYONIC_STEM_CELL_CORE & 323 & -0.57769 & -2.91488 & 0 \\
DANG_MYC_TARGETS_UP & 132 & -0.58907 & -2.70979 & 0 \\
HUPER_BREAST_BASAL_VS_LUMINAL_UP & 46 & -0.73074 & -2.70709 & 0 \\
RODRIGUES_THYROID_CARCINOMA_POORLY_DIFFERENTIATED_UP & 606 & -0.49343 & -2.6682 & 0 \\
FOURNIER_ACINAR_DEVELOPMENT_LATE_2 & 273 & -0.53196 & -2.66488 & 0 \\
RAMALHO_STEMNESS_UP & 197 & -0.53356 & -2.55144 & 0 \\
RHEIN_ALL_GLUCOCORTICOID_THERAPY_DN & 335 & -0.48737 & -2.4864 & 0 \\
BILD_MYC_ONCOGENIC_SIGNATURE & 195 & -0.51516 & -2.47975 & 0 \\
BIDUS_METASTASIS_UP & 204 & -0.50879 & -2.43919 & 0 \\
YAO_TEMPORAL_RESPONSE_TO_PROGESTERONE_CLUSTER_11 & 93 & -0.57496 & -2.43622 & 0 \\
RICKMAN_TUMOR_DIFFERENTIATED_WELL_VS_MODERATELY_DN & 82 & -0.57284 & -2.37207 & 0 \\
ELVIDGE_HYPOXIA_DN & 135 & -0.50709 & -2.28979 & 0 \\
KEGG_RIBOSOME & 85 & -0.55021 & -2.28603 & 0 \\
GROSS_HYPOXIA_VIA_HIF1A_UP & 74 & -0.55224 & -2.2832 & 0 \\
RICKMAN_TUMOR_DIFFERENTIATED_WELL_VS_POORLY_DN & 335 & -0.42365 & -2.15112 & $6.24 E-05$ \\
\hline
\end{tabular}

\section{I}

GSEA Enrichement in sip63 L3.6 cells compared to siControl

\begin{tabular}{lcccc}
\hline NAME & SIZE & ES & NES & FDR q-val \\
\hline LIN_SILENCED_BY_TUMOR_MICROENVIRONMENT & 87 & 0.741889 & 3.093567 & 0 \\
SENGUPTA_NASOPHARYNGEAL_CARCINOMA_DN & 184 & 0.56477 & 2.63524 & 0 \\
LIM_MAMMARY_STEM_CELL_DN & 391 & 0.490658 & 2.472543 & 0 \\
CHARAFE_BREAST_CANCER_LUMINAL_VS_MESENCHYMAL_UP & 399 & 0.492597 & 2.471339 & 0 \\
LIM_MAMMARY_LUMINAL_MATURE_UP & 82 & 0.584894 & 2.398431 & 0 \\
BOSCO_EPITHELIAL_DIFFERENTIATION_MODULE & 46 & 0.657135 & 2.390054 & 0 \\
SMID_BREAST_CANCER_RELAPSE_IN_BONE_UP & 55 & 0.598831 & 2.281816 & $7.76 E-05$ \\
CROMER_TUMORIGENESIS_DN & 34 & 0.64979 & 2.244613 & $7.33 E-05$ \\
ODONNELL_TARGETS_OF_MY_AND_TFRC_UP & 53 & 0.596211 & 2.233752 & $5.5 E-05$ \\
SMID_BREAST_CANCER_LUMINAL_B_UP & 103 & 0.523645 & 2.228037 & $5.28 E-05$ \\
SMID_BREAST_CANCER_BASAL_DN & 438 & 0.43851 & 2.226336 & $5.07 E-05$ \\
VECCHI_GASTRIC_CANCER_ADVANCED_VS_EARLY_DN & 93 & 0.527829 & 2.206488 & 7 E-05 \\
LIEN_BREAST_CARCINOMA_METAPLASTIC_VS_DUCTAL_DN & 79 & 0.533576 & 2.177088 & 0.000105 \\
CHARAFE_B_EEAST_CANCER_LUMMINAL_VS_BASAL_UP & 295 & 0.440284 & 2.153986 & 0.000178 \\
DAUER_STAT3_TARGETS_DN & 44 & 0.558594 & 2.014827 & 0.000859 \\
MARTENS_TRETINOIN_RESPONSE_UP & 271 & 0.412329 & 1.97972 & 0.001319 \\
BIDUS_METASTASIS_DN & 115 & 0.433148 & 1.864891 & 0.003689 \\
\hline
\end{tabular}

Figure 18 : Depletion of deltaNp63 hampers growth and reverses enrichment of gene expression profiles associated with the squamous subtype. (A) Gene expression analysis of deltaNp63 upon depletion of p63 after $48 \mathrm{~h}$ shown as relative mRNA expression and normalized to the unregulated housekeeping gene (GAPDH). $\mathrm{n}=3$. * P-value $<=0.05,{ }^{* *} \mathrm{P}$-value $<=0.01,{ }^{* * *} \mathrm{P}$-value $<=0.001,{ }^{* * * *} \mathrm{P}$-value $<=0.0001$. (B) Western blot analysis for p63 in L3.6pl and BxPC-3 $48 \mathrm{~h}$ after depletion of p63 to validate its downregulation. HSC70 is shown as loading control. (C-D) Crystal violet staining showing the proliferation of cells after $48 \mathrm{~h}$ of depletion of p63 compared to control for L3.6pl (C) and BxPC-3 (D) with relative area fraction shown in the bar graph. Data are represented as mean \pm SEM. $\mathrm{n}=2$. ${ }^{*} \mathrm{P}$-value $<=0.05,{ }^{* *} \mathrm{P}$-value $<=0.01,{ }^{* * *} \mathrm{P}$-value $<=0.001,{ }^{* * * *} \mathrm{P}$-value $<=0.0001$. (E-F) Sphere formation assay analysis with $E$ showing a representative change in the sphere structure upon p63 depletion and $F$ showing the numbers of the spheres formed in L3.6pl and BxPC-3 after 500 cells were seeded in 96-well plate for 7 days. Data are represented as mean \pm SEM. $n=24$. ${ }^{*}$ P-value $<=0.05$, ${ }^{* *} P$-value $<=0.01,{ }^{* * *} P$-value $<=0.001$, ${ }^{* * * *}$ $P$-value $<=0.0001$. (H-I) GSEA report for enriched genes upon downregulation of p63 in L3.6pl with pathways enriched in the control (E) and sip63 (F). 


\subsubsection{DeltaNp63 exerts its effects through activation of super enhancers}

To elucidate the mechanism by which deltaNp63 exerts its marked effect on cell proliferation, gene activation and pancreatic cancer cell fate specification, we examined the occupancy of deltaNp63 throughout the genome and identified numerous deltaNp63-occupied regions $(20,679$ peaks). Many of these regions intersected with H3K27ac and open chromatin regions identified by Assay for Transposase-Accessible Chromatin (ATAC) sequencing (Figure 19A, Supplementary Figure S2A). Interestingly, very few of these regions were associated with transcriptional start sites (TSS) and GREAT analysis revealed that the majority of deltaNp63 peaks were distal (Figure 19B, Supplementary Figure S2B, S2C). This distal pattern of occupancy implied that deltaNp63 mainly exerts its effects via enhancer activation. As depletion of deltaNp63 severely affects the transcriptional program of the cells and dramatically alters their molecular identity, we hypothesized that deltaNp63 may occupy and potentially nucleate super enhancers (SEs), as these have been reported to be major drivers of cell identity [131]. In concordance with the different gene activation profiles of Panc-1 compared to L3.6pl and BxPC3, distinct super enhancers were identified in Panc-1 compared to the other two cell lines, which generally showed the same patterns and tendencies (Figure 19C, Supplementary Figure S2D, S2E). Interestingly, the majority of super enhancers in L3.6pl contained at least one peak of deltaNp63, with approximately a quarter of them having more than two peaks (Figure 19E).

Taking into consideration the potential bias in identifying super enhancers, which is dependent on the stitching of regions and the intensity of the factor used to rank the enhancers [304], we compared these results using a new algorithm to identify clusters of regulatory elements (COREs). COREs are determined using a machine learning approach to consider different windows between enhancers for stitching and does not require intensity of factors for ranking enhancers (https://www.biorxiv.org/content/early/2018/03/20/222562). Interestingly, we 
observed a high overlap between COREs and SEs, with COREs also showing the same high degree of occupancy by deltaNp63 (Figure 19D, 19F). Notably, COREs and/or SEs containing more than two peaks of deltaNp63 displayed a particularly high dependence on deltaNp63 (Figure 19G, 19I). Notably, ChEA and enrichR consensus predicted p63 to be an upstream activator of super enhancers and highly significant ontology terms associated with super enhancers included squamous cell carcinoma, confirming the role of SEs in defining the squamous subtype (Supplementary Figure S2G, S2H). Concordantly, deltaNp63-dependent genes associated with SEs and/or COREs included genes that are associated with epidermal differentiation like keratins and integrins (Figure 19H). To identify the super enhancer regions that are driven by deltaNp63, we intersected the super enhancer regions in L3.6pl with the gained H3K27ac regions compared to Panc-1 in the both squamous cell lines (identified in Figure 17C) as well as the super enhancers identified in the patient-derived xenograft samples, since these more accurately represent in vivo squamous-like pancreatic tumors (Figure 19J). Consequently, we identified 93 SEs that were common for all these regions. We further filtered the SEs that were specifically enriched compared to the other patient-derived xenografts which clustered separately from the squamous samples and did not express deltaNp63 (Figure 19I). In this way we identified 45 super enhancer regions that were associated with the squamous subtype with high confidence. Interestingly, most genes associated with these regions showed a significant dependence on deltaNp63. These genes included most notably FAT Atypical Cadherin 2 (FAT2), Nectin Cell Adhesion Molecule 1 (NECTIN1) and Hypoxia Inducible Factor Alpha Subunit (HIF1A). These findings are in concordance with a squamous phenotype where hypoxic pathways were reported to be enriched and adhesion factors play a role in the development of the aggressive phenotype [215]. 
A

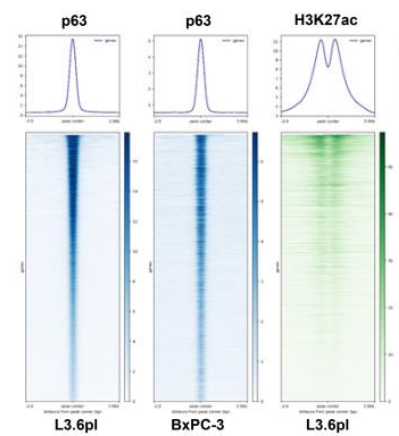

D

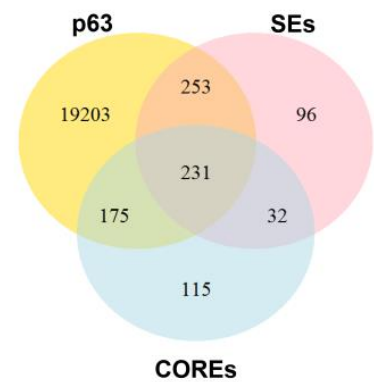

G

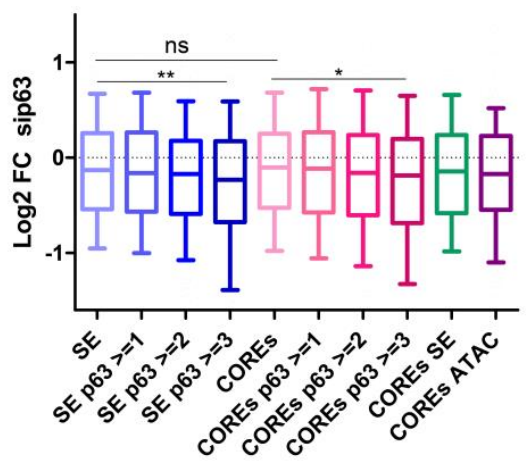

J H3K27ac_Gain

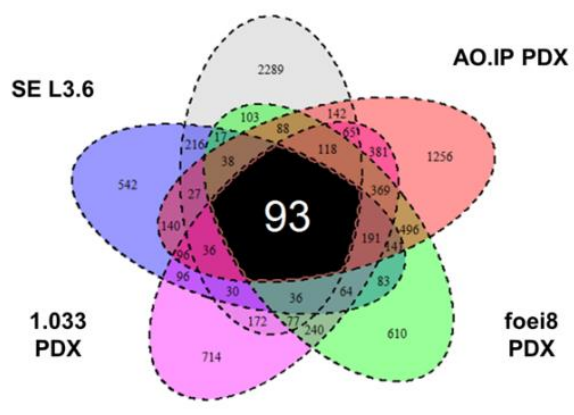

K $2.116 \mathrm{PDX} \quad 93 \mathrm{p} 63 \mathrm{SE}$

3.043 PDX 1095,14040

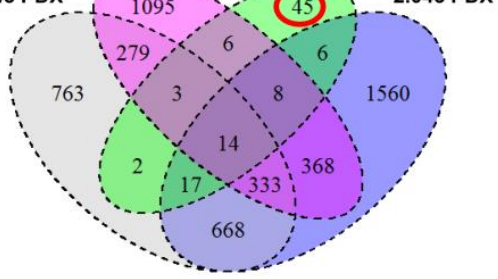

B C
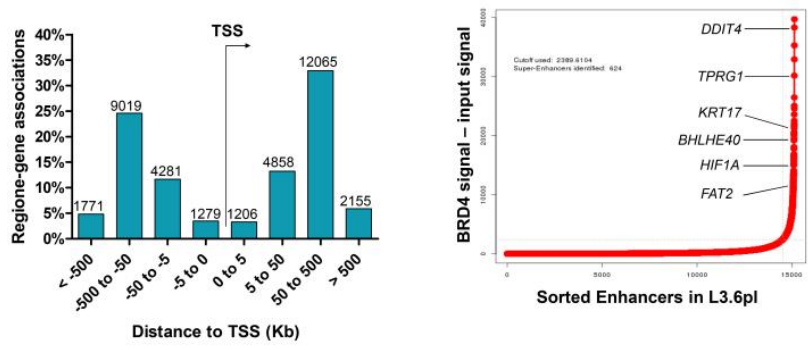

$\mathbf{F}$

E

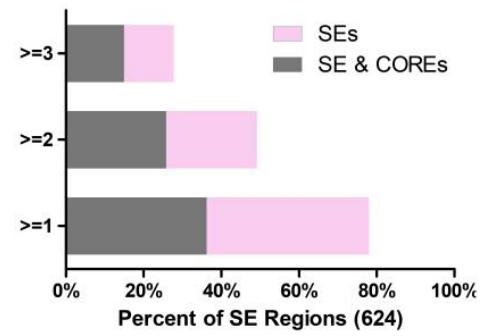

H

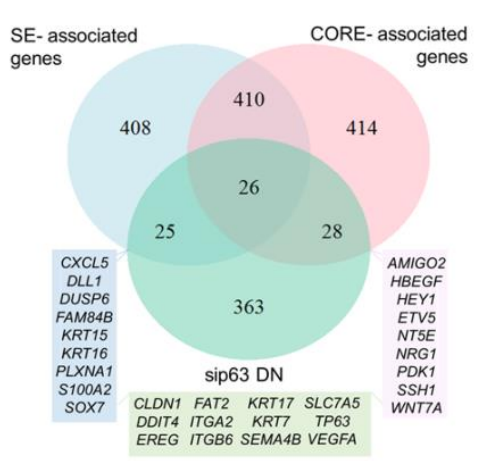

$\begin{array}{llll}\text { DDIT4 } & \text { ITGA2 } & \text { KRTT TP63 } \\ \text { EREG } & \text { ITGB6 SEMA4B VEGFA }\end{array}$

$\mathbf{L}$

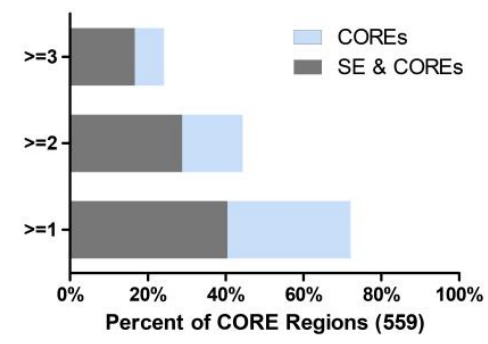

I

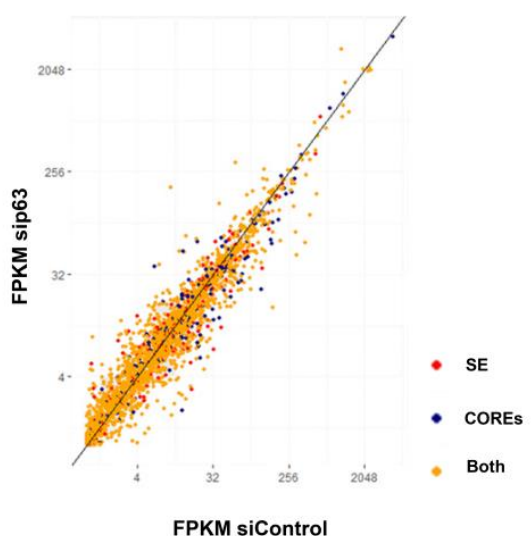

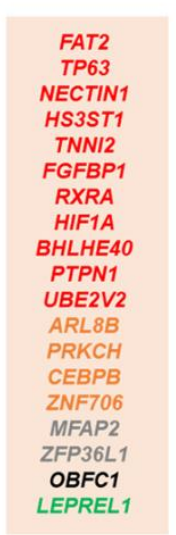

L3.6pl

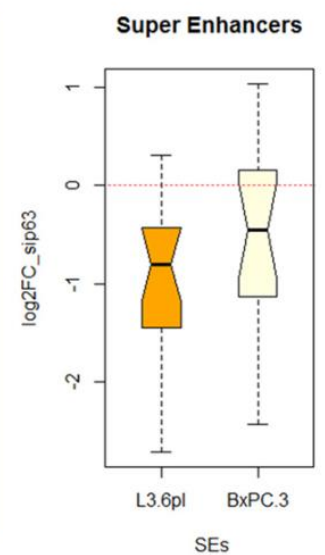

BxPC-3 
Figure 19 : DeltaNp63 exerts its effects through activation of super enhancers. (A) Average binding profiles and heatmaps depicting the p63, H3K27ac occupancy, and open chromatin defined by ATACseq at the p63 regions in L3.6pl. (B) Bar graph from GREAT analysis showing the distribution of p63 peaks of L3.6pl in relation to the TSS. (C) Enhancers in L3.6pl ranked based on BRD4 signal intensity using the ROSE algorithm defining 624 super enhancers. (D) Venn diagram showing the overlap between super enhancers defined by ROSE, clusters of regulatory elements by CREAM, and p63 peaks. (E-F) Bar graph showing the percentages of SEs (E) and COREs (F) that are occupied by any number of peaks of deltaNp63 (>=1), by at least more than 2 peaks, or 3 peaks, with grey depicting regions that are common between SEs and COREs. (G) Box plot showing the log2 fold change upon depletion of p63 for genes associated with SEs and COREs (defined based on H3K27ac or ATAC peaks) and their subgroups based on the number of p63 peaks they contain. (H) Venn diagram showing the overlap between genes associated with SEs and COREs and genes downregulated upon depletion of p63 (qvalue $=<0.05$, Fold change $=<-2$ ). Associated genes were identified by GREAT analysis using the basal plus extension association rule using default settings. (I) Scatter plot for FPKM values in control and sip63 samples for genes associated with super enhancers (red), COREs (blue), and both (yellow). (J-K) Venn diagrams depicting the overlap between H3K27ac gained regions, super enhancers in L3.6 and the three squamous xenografts in $\mathrm{J}$ and the common regions between them with the SEs of other xenografts lacking deltaNp63. (L) Box plot showing the log fold change upon depletion of p63 in the 45 super enhancer regions identified in $\mathrm{K}$ with the gene name on the left for L3.6pl and right for BxPC-3. Genes in red are significantly downregulated (q-value $<0.05$ ) by $>1.5$ fold, orange by 1.2-1.5 fold, green are significantly upregulated and grey indicate insignificant changes.

\subsubsection{Super enhancers in the squamous subtype are highly dependent on}

\section{deltaNp63}

To validate that the super enhancers which we identified are dependent on deltaNp63, we performed chromatin immunoprecipitation followed by quantitative real-time PCR on selected regions in those enhancers after depletion of deltaNp63. Specifically, we examined enhancers associated with FAT2, NECTIN1, and HIF1A due to their high dependence on deltaNp63 and their high relevance to the squamous phenotype. We observed two regions that are upstream of FAT2 which were occupied by a peak of p63 in two separate SEs and corresponding to ATAC peaks in L3.6pl and H3K27ac peaks in L3.6pl, BxPC-3 and the three squamous patient-derived xenografts (Figure 20A). We validated the downregulation of these genes in both L3.6pl and BxPC-3 by qRT-PCR and the occupancy of these regions by deltaNp63, which was lost upon its depletion (Figure 20B, 20C). 
A

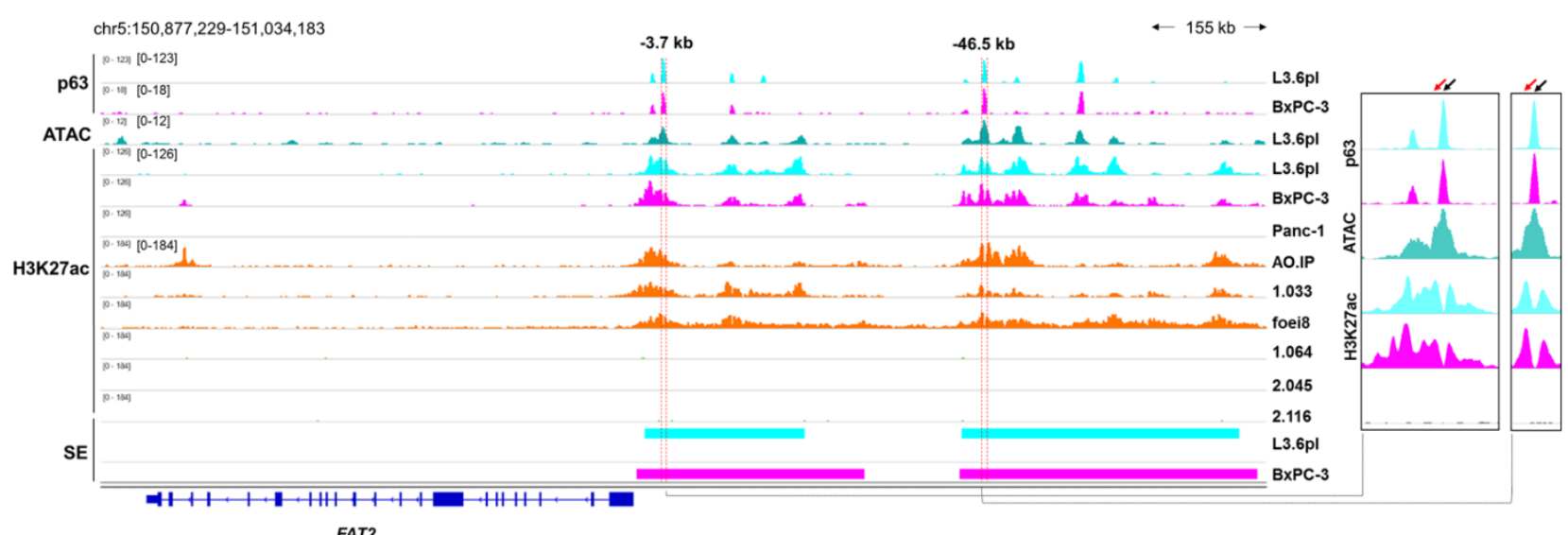

B

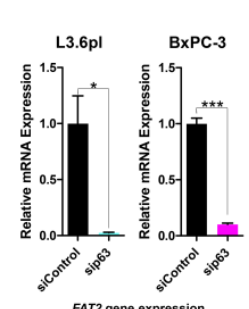

FAT2 gene expression
C

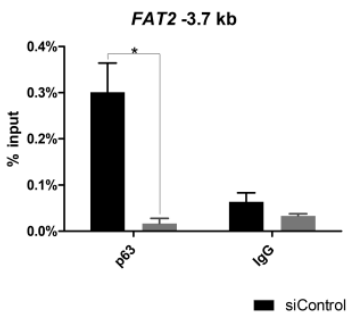

p63 ChIP

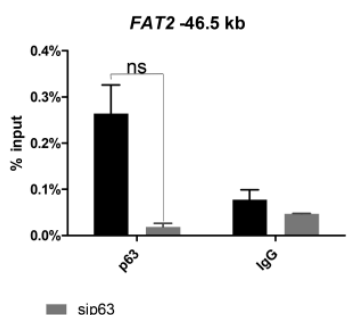

D

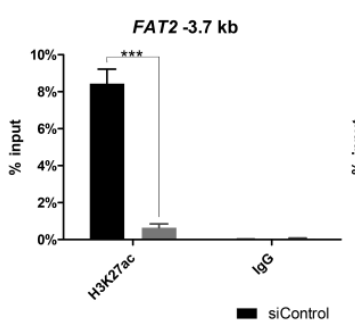

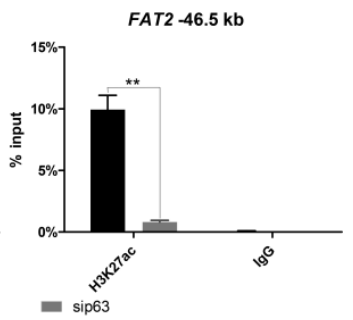

E

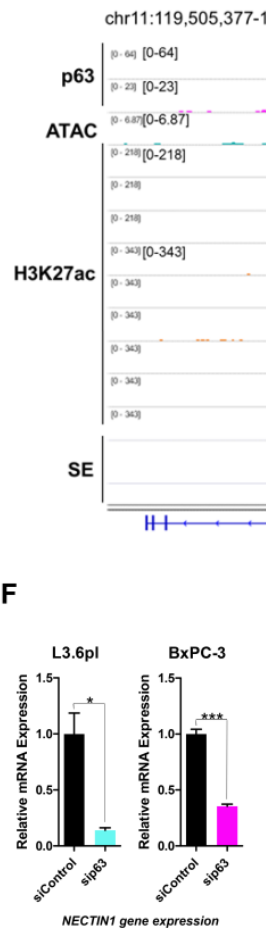

14,709

$+36.2 \mathrm{~kb}+31.8 \mathrm{~kb}$

$\leftarrow 108 \mathrm{~kb} \rightarrow$

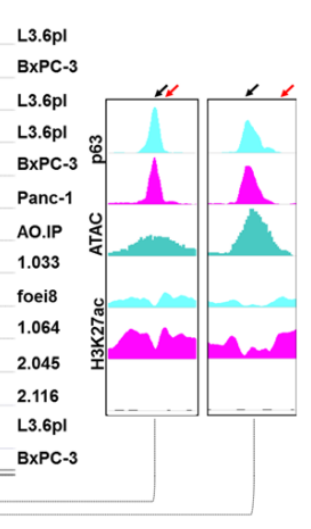

NECTIN1

G

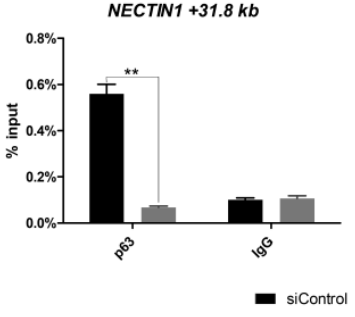

p63 ChIP

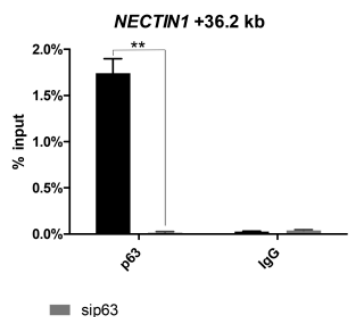

H

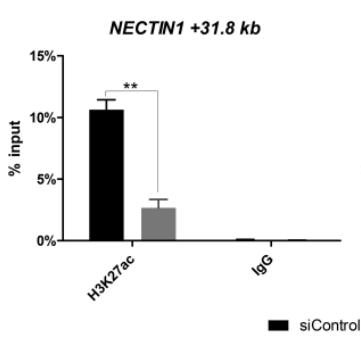

H3K27ac ChIP

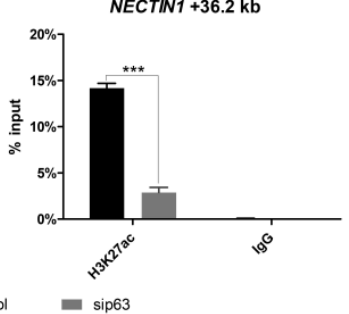


Figure 20 : Super enhancers in the squamous subtype are highly dependent on deltaNp63. (A) Occupancy profiles at the FAT2 gene, which was identified to be highly dependent on deltaNp63 and associated with subtype-specific SEs. Profiles shown are for p63 in L3.6pl and BxPC-3, ATAC-seq in L3.6pl, H3K27ac in L3.6pl and BxPC-3, and six xenografts (three in orange, which express deltaNp63expressing tumors, and three in green as a representative sample of the opposing clustering xenografts), in addition to the region files for SEs in L3.6pl and BxPC-3. Enlarged snapshots of the regions highlighted by the red dotted lines are shown on the right with black arrows showing the regions examined for p63 enrichment and the red arrows for H3K27ac. (B) Gene expression analysis for FAT2 following depletion of p63 for $48 \mathrm{~h}$ shown as relative mRNA expression and normalized to an unregulated housekeeping gene (GAPDH). $\mathrm{n}=3 .{ }^{*} \mathrm{P}$-value $<=0.05,{ }^{* *} \mathrm{P}$-value $<=0.01,{ }^{* * *} \mathrm{P}$-value $<=0.001,{ }^{* * *} \mathrm{P}$-value $<=0.0001$. (C-D) Validation of p63 (C) and H3K27ac (D) enrichment by ChIP-qPCR at the two highlighted regions in A (-3.7 $\mathrm{kb}$ from TSS of FAT2 and $-46.5 \mathrm{~kb}$ from TSS of FAT2) in control and p63-depleted cells after $48 \mathrm{~h} . \mathrm{n}=2-3$. Data are represented as mean \pm SEM. $n=2$. ${ }^{*} P$-value $<=0.05,{ }^{* *} P$-value $<=0.01,{ }^{* * *} P$-value $<=0.001$, **** P-value $<=0.0001$. (E-F) Same as in (A-B) at the NECTIN1. (G-H) Same as in (C-D) at the highlighted regions for NECTIN1 (+31.8 kb and $+36.2 \mathrm{~kb}$ from TSS of NECTIN1).

\subsubsection{DeltaNp63 cooperates with other transcription factors to activate target}

\section{genes associated with worse prognosis}

Given the crucial role of deltaNp63 in defining a tumor subtype characterized by poorer prognosis, we evaluated the association of the deltaNp63-dependent SE-associated genes with prognosis using data from the TCGA research network (http://cancergenome.nih.gov). Interestingly, increased expression of most of these deltaNp63-dependent genes displayed a significant correlation with poorer prognosis in pancreatic cancer patients (Figure 21A, Supplementary Figure 4). As transcription factors frequently function cooperatively in lineage specification [98], we examined the list of genes associated with our identified enhancers and evaluated the expression of transcription factors contained within that list using the Morpheus Database. Remarkably, the transcription factors HIF1A, Basic Helix-Loop-Helix Family Member E40 (BHLHE40), and Retinoid X Receptor Alpha (RXRA) were more highly expressed in L3.6pl and BxPC-3 cells compared to Panc-1 (Figure 21B). Consequently, we asked if this specific expression pattern may help to form a transcriptional network underlying the marked effects of deltaNp63 in our system. Accordingly, we utilized genome occupancy data for HIF1A, RXRA, and BHLHE40 from the ReMAP database and filtered out all regions that did not overlap with the gained H3K27ac regions in L3.6pl and BxPC-3 compared to Panc1 (Fold change > 4, FDR 
$<0.5)$. Then, using this information, together with our RNA-seq results, we constructed a regulatory network containing deltaNp63-dependent associated genes, along with the deltaNp63-dependent SE-associated genes, and extended the network by transcription factortarget query function using the Cytoscape iRegulon app (Figure 21C). Notably, many of the target genes were affected by a combination of these transcription factors, which also showed reciprocal regulation patterns with many of the transcription factors binding and activating one another. Accordingly, we conclude that deltaNp63 drives the expression of some central target genes via the activation of super enhancers associated with downstream transcription factors such as HIF1A, BHLHE40, and RXRA. The activation of these deltaNp63-dependent genes enables the further indirect or cooperative activation of additional downstream target genes.

\subsection{Discussion}

Gene expression and epigenetic profiles in cancer cells can be affected by many factors that are intrinsic and extrinsic to the tumor. This renders the investigation of molecular subtypes in malignancies quite challenging as systems to study the molecular mechanisms behind these subtypes are scarce. In this study, we were able to discern the same patterns of molecular subclasses observed in patients in both cell lines and patient-derived xenografts [214, 215]. This confirms the high reproducibility of these stratifications and implies that these molecular characteristics are highly conserved and can withstand extreme changes of conditions. Most importantly, these systems provide an ideal opportunity to identify and target certain dependencies specific for the more aggressive subtypes. L3.6pl is unique as it exhibits highly metastatic characteristics due to the repeated cycles of spontaneous liver metastasis that it underwent during its establishment [378]. Thus, it is not surprising that this cell line was found to be one of the systems that is representative of the squamous subtype, as it is one of the most highly metastatic and aggressive pancreatic cell lines [379]. 
A

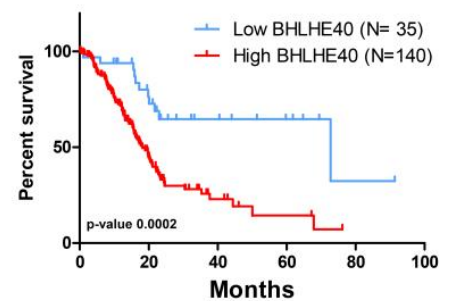

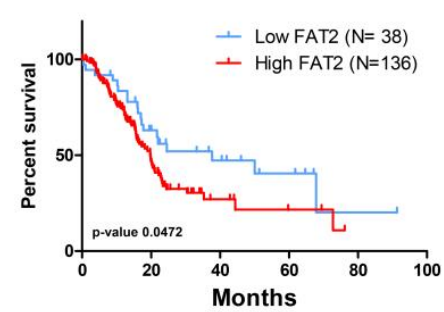
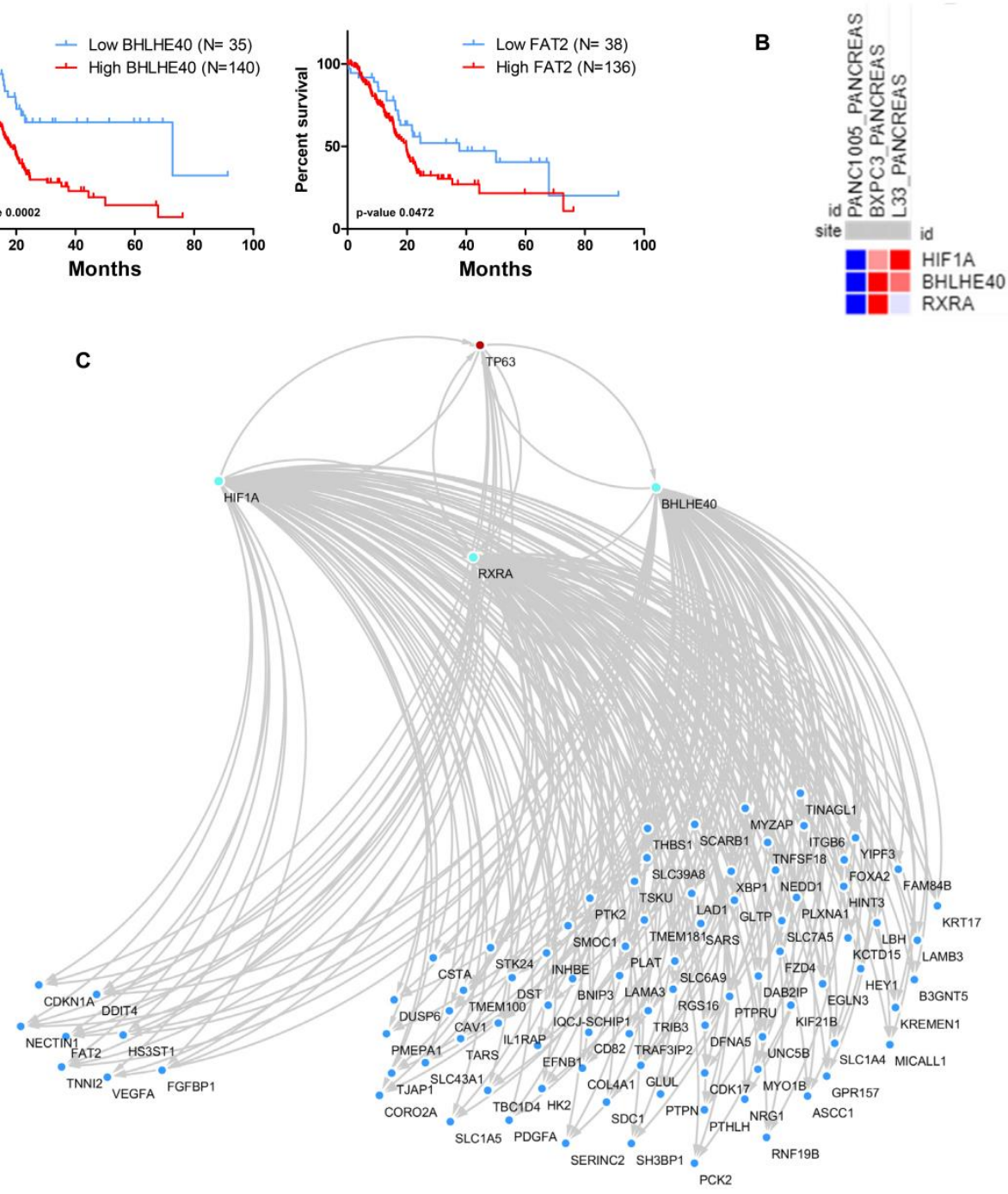

D

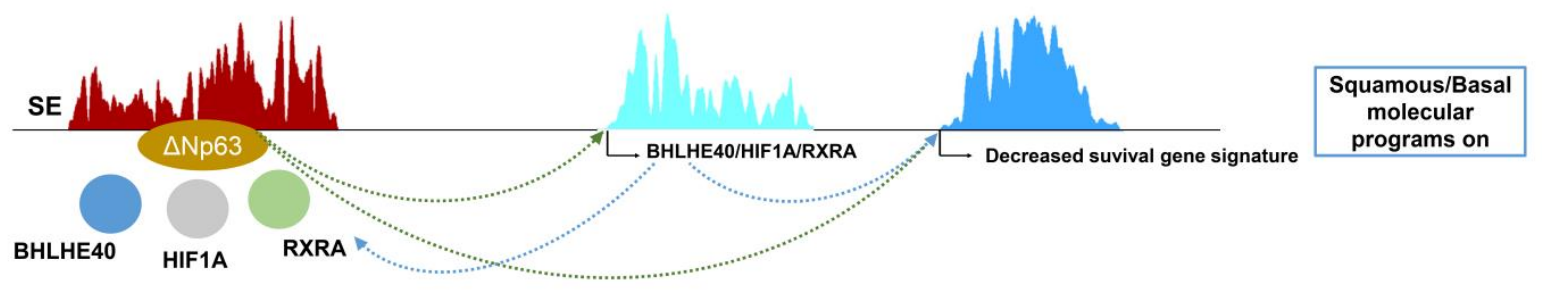

set

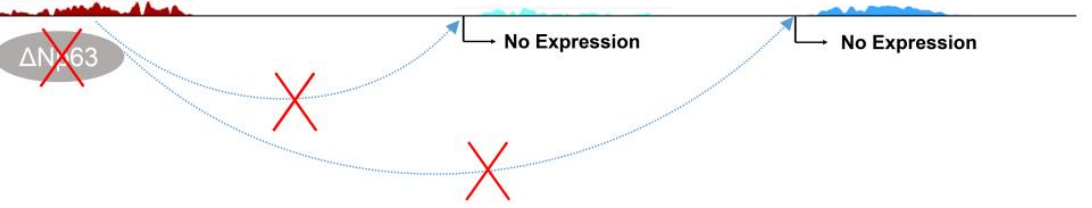

Squamous/Basal

molecular

programs off 
Figure 21 : DeltaNp63 and its target transcription factors. (A) A Kaplan-Meier plot showing the percent survival in pancreatic cancer patients (TCGA database) expressing high and low levels of BHLHE40 and FAT2 with p-value indicated on graph. (B) Heatmap depicting the gene expression patterns of transcription factors included in $4 \mathrm{~L}$, namely HIF1A, BHLHE40, and RXRA in different pancreatic cancer cell lines (Morpheus database). (C) Network depicting the interactions of HIF1A, BHLHE40, RXRA, and p63, where common genes are activated by all those transcription factors, which also activate each other. TF-target interactions were extracted from iRegulon app of Cytoscape in addition to the genes associated with peaks from these transcription factors that intersect with H3K27ac gained regions (with a lower fold change threshold of 4). (D) A graphical presentation of the mechanism by which deltaNp63 drives gene expression programs associated with a poorer prognosis by activating subtype-specific super enhancers and activation of downstream transcription factors that, in turn, further activate additional downstream enhancers and genes.

Notably, utilization of differentially active regions identified in the cell lines representative of the squamous/basal-like subtype (Supplementary Table S4) helped to successfully identify a subgroup of patient-derived xenografts which were, consistently, previously classified as squamous/basal by Lomberk et al. using other criteria [220]. Additionally, the samples which clustered the closest to the squamous triad were also identified as basal, supporting a high degree of similarity between the basal and squamous classifications. Molecular subtyping of pancreatic cancer currently requires the use of bioinformatically complicated algorithms and are usually not particularly robust as demonstrated by only partially overlapping results seen in major recent studies [213-215, 368]. One reason for the apparent discrepancies may be due to tumor subgroups within the larger subgroups. Indeed, our analysis implicates deltaNp63 as a major driver of gene activation defining a squamous subgroup contained within the larger basal subgroup. Thus, deltaNp63 expression may be a defining feature of a further unappreciated subgroup of basal-like pancreatic tumors expressing a more squamous gene expression signature. In this study, we report a directed approach which involves principle component analysis of a single epigenetic marker (H3K27ac) on a select set of enhancer regions that are differentially active in the squamous/basal subgroup and which successfully clustered PDX samples based on molecular subtypes. This serves as an example for an accessible method to identify regions and gene signature patterns in various samples. Future studies in the scope of 
molecular subtypes of pancreatic cancer will play an important role in introducing conformity and clarity to the currently diverse subtyping approaches based largely on gene expression patterns.

In this study, we were able to define subtype-specific super enhancers (Supplementary Table S5) associated with the aggressive squamous subtype in a manner akin to lineage-specific enhancers defining cell fate in pluripotent cells [380]. Consistently, our findings uncover a tightly intertwined transcriptional network downstream of deltaNp63 which resembles what has been reported for transcription factors controlling pluripotency [381-383]. Accordingly, it is evident that programming of cell fates, molecular subtypes and phenotypes is efficiently achieved using a collection of transcription factors, whereby the tight regulation of whole gene expression programs is controlled by a distinct set of master transcription factors. The identification of transcription factors that are both dependent on and activate deltaNp63 in the squamous subtype can help in optimizing therapy and shed light on the molecular mechanisms which define the squamous/basal-like subtype.

Consistent with our findings, hypoxic pathways were previously reported to be enriched in the squamous subtype [215], although a direct connection to deltaNp63 was not known. Given the major role of HIF1A in the response to hypoxia, it appears likely that it may also function in promoting the increased aggressiveness of the squamous subtype and promoting cellular plasticity under hypoxic conditions [384-386]. Less is known about the role of RXRA and BLHLE4O in pancreatic cancer. BHLHE40 was found to play a crucial role in promoting a molecular switch to pro-inflammation in T-helper cells [387]. In the brain, BHLHE40 plays a role in promoting synaptic plasticity [388]. These roles in other systems imply that BHLHE40 may also play a role in promoting plasticity and leading to a poorer outcome. RXRA forms a heterodimer with peroxisome proliferator-activated receptors (PPARs) which can be targeted by PPAR inhibitors [389]. RXRA also dimerizes with the vitamin D receptor and its mutation is 
associated with bladder cancer and melanoma $[389,390]$. Further studies will uncover if optimal treatment of the more aggressive subtype may include the pathways that are regulated through these transcription factors.

Based on our work, the primary mode of activation of the deltaNp63-associated transcription factor network appears to be at distal regulatory elements whereby the factors not only promote the expression of common target genes but also control the expression of one another. This enhancer-specific effect is supported by our observation that differentially active regions in L3.6pl and BxPC-3 compared to Panc-1 were mainly found at putative enhancer regions and not at transcriptional start sites. Moreover, deltaNp63 occupancy at enhancers, but not at TSS regions, was more closely correlated with the effects of p63 depletion on target gene expression. Altogether, our findings underscore the importance of distal regulatory elements in driving important transcriptional programs in tumorigenesis and tumor progression, thereby providing a further rationale for targeting these regions and their dependencies.

We have identified a marked dependence of a subset of super enhancers on deltaNp63 which may open the door for specific targeting of the squamous subtype of pancreatic cancer. However, our findings also confirm the tremendous plasticity of pancreatic cancer, where a single factor is required for the activation of a whole gene signature associated with a poorer outcome. Despite the fact that cancer is characterized by inter- and intra-heterogeneity, distinct patterns of gene activation still emerge and may imply a natural selection process where certain attributes, such as overexpression of deltaNp63, lead to the selective growth of these more aggressive and highly pliable tumor cells. It is likely that similar selective pressures will occur as we target the dependencies of the subtype-specific enhancers since the activation of other factors may likely lead to the activation of other compensatory gene expression programs. Future studies will be necessary to determine which factors specifically determine the gene 
expression patterns and cellular phenotypes of other pancreatic cancer subtypes. It will then be possible to examine the biological and therapeutic effects of subtype switching and determine whether such approaches may be useful in a therapeutic setting.

\subsection{Materials and Methods}

\subsubsection{Cell culture and siRNA transfections}

L3.6pl cells [378] were cultured in phenol-free minimum essential medium (MEM; Thermo Fischer Scientific) supplemented with 10\% FBS, 1\% penicillin/streptomycin, and 1\% Glutamine. Capan-1, BxPC-3, and MIA PaCa-2 were maintained in Roswell Park Memorial Institute medium (RPMI 1640; Thermo Fischer Scientific) supplemented as MEM. Panc-1, PaTu 8902, and DanG cells were maintained in high glucose GlutaMAX Dulbecco's Modified Eagle Medium (DMEM; Thermo Fischer Scientific) supplemented with $10 \%$ FBS and $1 \%$ penicillin/streptomycin. siGENOME SMARTpool siRNA (Dharmacon) were used to deplete p63 (D-003330-05, D-003330-06, D-003330-07, D-003330-08; respective target sequences: CAUCAUGUCUGGACUAUUU, CAAACAAGAUUGAGAUUAG, GCACACAGACAAAUGAAUU, CGACAGUCUUGUACAAUUU). Control siRNA used was siGENOME Non-Targeting \#5 (NT5; Target sequence: UGGUUUACAUGUCGACUAA). Transfections were performed using Lipofectamine ${ }^{\circledR}$ RNAiMAX (Invitrogen) according to the manufacturer's instructions.

\subsubsection{Proliferation and sphere formation assays}

100,000 (L3.6pl) or 50,000 (BxPC-3) cells were reverse transfected in duplicates in 12- or 24well plates, respectively, with NT5 or p63 siRNAs. After 48 h, cells were fixed with methanol for 10 min and stained with $1 \%$ crystal violet in $20 \%$ ethanol for $20 \mathrm{~min}$ and then washed and scanned. Relative area fraction was measured using ImageJ and plotted using GraphPad Prism 
version 5.04 (GraphPad Software, Inc.). For sphere formation assay, cells were transfected on day 1 as previously mentioned and in duplicates for NT5 and p63 and then 500 cells were seeded in 96-well plate ( $n=12$ for each duplicate, $n=24$ for each condition). Plates were scanned after 7 days by Celigo® $\mathrm{S}$ imaging cytometer (Nexcelom Bioscience LLC).

\subsubsection{Protein extraction and western blot analysis}

Protein was extracted by washing cells with PBS and suspending in RIPA buffer (1X PBS, 0.5\% sodium deoxycholate $0.1 \%$ SDS, $1 \%$ NP-40) supplemented with $100 \mu \mathrm{M} \beta$-glycerophosphate disodium salt hydrate (BGP), $100 \mu \mathrm{M}$ N-ethylmaleimide, and protease inhibitors (100 $\mu \mathrm{M}$ Pefabloc, $1 \mu \mathrm{M}$ aprotonin, $1 \mu \mathrm{M}$ leupeptin). Protein lysates were solubilized by sonication using a Bioruptor Pico (Diagenode) for 10 cycles (30 s on/off). Laemli buffer (375 mM Tris/ $\mathrm{HCl}, 10 \%$ SDS, 30\% glycerol, $0.02 \%$ bromophenol blue, $9.3 \%$ DTT) was added to lysates before separation with a $7 \%$ polyacrylamide gel for evaluation of expression of the levels of $p 63$ in multiple pancreatic cell line and $12 \%$ for p63 knockdown validation. Protein was then transferred onto nitrocellulose membranes that were incubated with primary antibodies in $5 \%$ milk in TBS-T overnight and then with secondary antibodies for one hour. Protein bands were visualized using Bio-Rad ChemiDocTM imager. Antibodies used were: HSC70 Santa-Cruz (\#sc-7298) in 1:10,000 dilution and p63 (4A4) Santa-Cruz (\#sc-8431) in 1:1000.

\subsubsection{RNA isolation and quantitative real-time PCR (qPCR)}

RNA isolation and quantitative real-time PCR was performed as previously described [330, 391]. Briefly, triplicates for each condition were harvested $48 \mathrm{~h}$ after transfection by QIAzo|® reagent (Qiagen). Reverse transcription of $1 \mu \mathrm{g}$ RNA was performed using M-MuLV reverse transcriptase (NEB) and random primers and the resultant complementary DNA was quantified by quantitative real-time PCR (qPCR) using a CFX Connect ${ }^{\mathrm{TM}}$ Real-Time System (Bio-Rad). 
Gene expression levels were normalized relative to an internal unregulated reference gene, GAPDH. Protocol for qPCR runs included 2 min denaturation at $95^{\circ} \mathrm{C}, 40$ cycles of $10 \mathrm{~s}$ at $95^{\circ} \mathrm{C}$ followed by $30 \mathrm{~s}$ at $60^{\circ} \mathrm{C}$. Primers (Supplementary Table S1) were designed using the NCBI primerblast design tool (https://www.ncbi.nlm.nih.gov/tools/primer-blast/) and were ordered from Sigma-Aldrich (Germany).

\subsubsection{Chromatin immunoprecipitation (ChIP)}

Chromatin immunoprecipitation was performed as described previously [99, 152]. Briefly, cells were crosslinked with $1 \%$ formaldehyde for 20 minutes and quenched by glycine $(125 \mathrm{mM}$ final concentration). Cells were scraped and nuclear pellets were prepared in and washed with the nuclear preparation buffer (150 mM NaCl, $20 \mathrm{mM}$ EDTA, $50 \mathrm{mM}$ Tris- $\mathrm{HCl}(\mathrm{pH} 7.5), 0.5 \% \mathrm{v} / \mathrm{v}$ NP-40, 1\% v/v Triton-X-100, $20 \mathrm{mM} \mathrm{NaF).} \mathrm{Samples} \mathrm{were} \mathrm{then} \mathrm{sonicated} \mathrm{in} \mathrm{sonication} \mathrm{buffer}$ $(150 \mathrm{mM} \mathrm{NaCl}, 20 \mathrm{mM}$ EDTA, $50 \mathrm{mM}$ Tris- $\mathrm{HCl}(\mathrm{pH} 8), 1 \% \mathrm{v} / \mathrm{v} \mathrm{NP}-40,0.5 \% \mathrm{v} / \mathrm{v}$ sodium deoxycholate, $20 \mathrm{mM} \mathrm{NaF}, 0.1 \%$ SDS) for 30 cycles (in L3.6pl) or 25 cycles (in BxPC-3 and Panc-1) using a Bioruptor Pico (Diagenode) and a cycle setting of $30 \mathrm{~s}$ on/off. Consequently, samples were precleared by incubation with $50 \%$ slurry of Sepharose 4B (GE Healthcare), centrifuged and supernatants were incubated with antibody overnight. Antibodies included p63

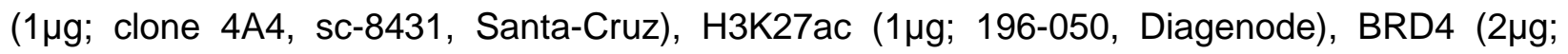

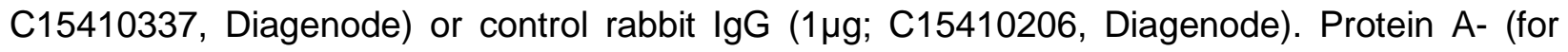
rabbit antibodies) or Protein G- (for mouse antibodies, 4A4 p63) Sepharose beads were added to samples and incubated for $2 \mathrm{~h}$, then washed, de-crosslinked, and DNA was extracted. For ChIP-sequencing, samples were performed in duplicate for each condition. For ChIP-qPCR for validation of enrichment and loss upon depletion of p63, cells were seeded in triplicate and transfected with siRNA and nuclear pellets harvested after $48 \mathrm{~h}$. Quantitative PCR conditions were the same as gene expression studies but the cycle number was increased to 46 . Primers 
were designed for regions of positive enrichment for p63 and H3K27ac and the first intron of OLIG2 was used as a negative site for enrichment to ensure specificity of signal (oligonucleotide sequences for ChIP validation can be found in Supplementary Table S1). The signal was normalized to input DNA and presented as percent input for duplicates or triplicates in each condition.

\subsubsection{Assay for transposase-accessible chromatin (ATAC)}

ATAC-seq was performed following the protocol of Buenrostro et al [392]. Briefly, 50,000 L3.6pl cells were trypsinized and washed twice with cold PBS. Then cells were re-suspended in lysis buffer (10mM Tris- $\mathrm{HCl} \mathrm{pH} 7.5,10 \mathrm{mM} \mathrm{NaCl}, 3 \mathrm{mM} \mathrm{MgCl}_{2}, 0.1 \%$ IGEPAL CA-630), incubated for $15 \mathrm{~min}$ on a rotating wheel at $4 \mathrm{C}$, followed by centrifugation and then resuspended in $50 \mu \mathrm{l}$ of transposition mix composed of $2.5 \mu$ of TDE1 (Nextera Tn5 Transposase), $25 \mu \mathrm{l}$ TD (2x reaction buffer) in nuclease free water (Nextera DNA Library Prep Kit, FC-121-1030, Illumina). DNA extraction was immediately preformed after 30 min of incubating the transposition reaction at 37 C. MinElute PCR Purification kit (Qiagen) was used for DNA extraction and following the instructions of the manufacturer. Experiment was done in duplicates.

\subsubsection{Library preparation for RNA, ChIP, and ATAC-seq and next-generation sequencing}

The integrity of RNA from L3.6pl and BxPC-3 with triplicates for siControl and sip63 were verified by agarose gel electrophoresis. Libraries for RNA were prepared using the TruSeq RNA Library Prep Kit V2 (Illumina) according to the manufacturer's instructions. Libraries for DNA from ChIP were made using the Microplex Library Preparation kit v2 (Diagenode) according to the manufacturer's instructions. ATAC libraries were made using the Nextera DNA Library Prep 
Kit and the libraries were amplified for 15 cycles in total of: $98 \mathrm{C}$ for $10 \mathrm{sec}, 63 \mathrm{C}$ for $30 \mathrm{sec}$, and $72 \mathrm{C}$ for $60 \mathrm{sec}$. The quality of the libraries was verified using the high sensitivity DNA kit (Agilent) on the Agilent Bioanalyzer 2100. RNA- and ChIP-seq samples were sequenced (single-end $50 \mathrm{bp}$ ) on a HiSeq4000 (Illumina) in the Transcriptome and Genome Analysis Laboratory (TAL) at the University Medical Center Göttingen. ATAC-seq samples were sequenced (single-end 50bp) on HiSeq2000 (Illumina). Images of sequences were converted into bcl files (BaseCaller software, Illumina) and demultiplexed to fastq files by CASAVA v1.8.2.

\subsubsection{Bioinformatic analysis for ChIP and ATAC-sequencing}

The quality of FASTQ files for ChIP- and ATAC-seq (Accession numbers in Supplementary Table S2) was checked using FASTQC/0.11.4 for data generated for this study and data from Lomberk et al. which were downloaded from ArrayExpress [220]. Data were mapped to the reference genome assembly (hg19) using BOWTIE2/2.2.5 [359] and converted to bam files and sorted and indexed using SAMTOOLS/1.4 [360]. Subsequently, reads were normalized to $1 \mathrm{X}$ sequencing depth using the bamCoverage tool in DEEPTOOLS/2.4.0 [361], ignoring duplicates and extending to $200 \mathrm{bp}$ (500 bp for ATAC-seq) to generate occupancy profiles that were viewed with the Integrative Genomics Viewer (IGV 2.4) [362, 363]. Peaks were called using MACS2/2.1.1.20160309 without building the shifting model and with cutoff of less than 0.05 (-broad-cutoff 0.05 for BRD4 and H3K27ac) and input files as background [393]. Hierarchical clustering was performed for H3K27ac regions by seqMINER/1.3.4 using KMeans enrichment linear as clustering normalization [394, 395]. Differential binding analysis was performed to identify differentially occupied regions in L3.6pl and BxPC-3 compared to Panc-1 using the Bioconductor $\mathrm{R}$ package Diffbind run on $\mathrm{R}$ version 3.3.1 according to the instruction manual [396]. Genomic Regions Enrichment of Annotations Tool (GREAT) analysis was used to identify associated genes with regions identified by differential binding analysis and hierarchical 
clustering. multiBigwigSummary BED-file and plotPCA tools in DEEPTOOLS/2.4.0 were used to plot the principle component analysis for the H3K27ac profiles on differentially occupied regions for the 24 patient-derived xenografts. Heatmaps and average profiles for occupancy were generated using the computeMatrix and plotHeatmap tools on the European UseGalaxy server and the reference point mode were selected as the peak center [397]. Super enhancers were identified using the ROSE algorithm by using the H3K27ac regions as input files and BRD4 compared to input as intensity files, ignoring regions that are 2500 bp around TSS and keeping stitching of regions to the default $12.5 \mathrm{~kb}[123,124]$. Cluster of regulatory elements (COREs) were identified using the CREAM R package according to instruction manual [398]. To identify common super enhancers, we used the multiinter tool in BEDTOOLS/2.24 and the VennDiagram R package to generate the Venn diagrams [399, 400]. Upstream activators for super enhancer regions were identified using EnrichR web-based interface [401]. Occupancy regions of HIF1A, BHLHE40, RXRA were extracted from the ReMAP database v1.2 $[402,403]$.

\subsubsection{Bioinformatic analysis for RNA-seq}

The quality for FASTQ files was evaluated as previously mentioned for datasets generated for this study in addition to the ones downloaded from Lomberk et al. and Mishra et al. (Accession numbers in Supplementary Table S3) [220, 330]. Reads were mapped using TOPHAT/2.1.0 and annotation file for hg19 was downloaded from the UCSC table browser [404, 405]. Fragments per kilobase per million (FPKM) values were calculated and differential gene expression analysis was performed using CUFFLINKS/2.2.1 [406]. Unexpressed genes with very low FPKM values in both conditions (siControl and sip63) were disregarded to avoid bias. Hierarchical clustering by Euclidean distance for FPKM and Z-score was performed using cluster 3.0 [407] and the resulting heatmaps viewed using TreeView 3.0 [408]. Z-scores were calculated by subtracting the mean of FPKM values in all cells and dividing by the deviation. FPKM values for 
expressed genes in any condition were used as input for gene set enrichment analysis (GSEA) which was performed using default settings (1000 permutations and for a maximum size of sets of 1000) [409]. For heatscatter plot for signal of p63 at TSS, the TSS with the highest signal was taken. The transcription factor and target gene network was visualized using Cytoscape v3.6.1 and extended by the TF-target query of the iRegulon app [410, 411].

\subsubsection{Statistical Analysis}

For patient survival curves, the Mantel-Cox test was used to evaluate significance. For sphere formation assays and FPKM values, the Mann-Whitney test was applied. For analysis of qPCR, a non-parametric $t$ test was used. $p$-values were symbolized by ${ }^{\star \star \star *}$ for $=<0.0001,{ }^{* \star *}$ for $=<$ $0.001,{ }^{* *}$ for $=<0.01$ and ${ }^{*}$ for $=<0.05$.

\subsection{Accession Numbers}

RNA-seq, ChIP-seq, and ATAC-seq profiles have been deposited at ArrayExpress (http://www.ebi.ac.uk/arrayexpress) and are available under the accession numbers E-MTAB7033, E-MATB-7034, and E-MTAB-7035.

\subsection{Acknowledgement}

The authors would like to thank G. Salinas, F. Ludewig, and S. Lutz from the Transcriptome and Genome Analysis Laboratory of the University Medical Center Göttingen, Germany for performing the next generation sequencing for ChIP-seq and RNA-seq data; the Fisher Lab at the German Center for Neurodegenerative Diseases for performing the next generation sequencing for ATAC-seq; and Z. Najafova, X. Wang, A. Kutschat, J. Henck and all the members of the Johnsen group for their support and helpful discussions. 


\subsection{Funding}

This work was supported by the German Academic Exchange Service (DAAD) to F.H.H.; Deutsche Krebshilfe (PiPAC Consortium) [70112505] and the Deutsche Forschungsgemeinschaft (DFG) [JO 815/3-2] to S.A.J. Funding for open access charge: Internal house funding/university funding.

\subsection{Conflict of interests}

The authors declare no conflict of interest. 


\subsection{Supplementary figures and tables}

\subsubsection{Supplementary Figure 1 (Figure 22)}

\section{Supplementary Figure 1}

A

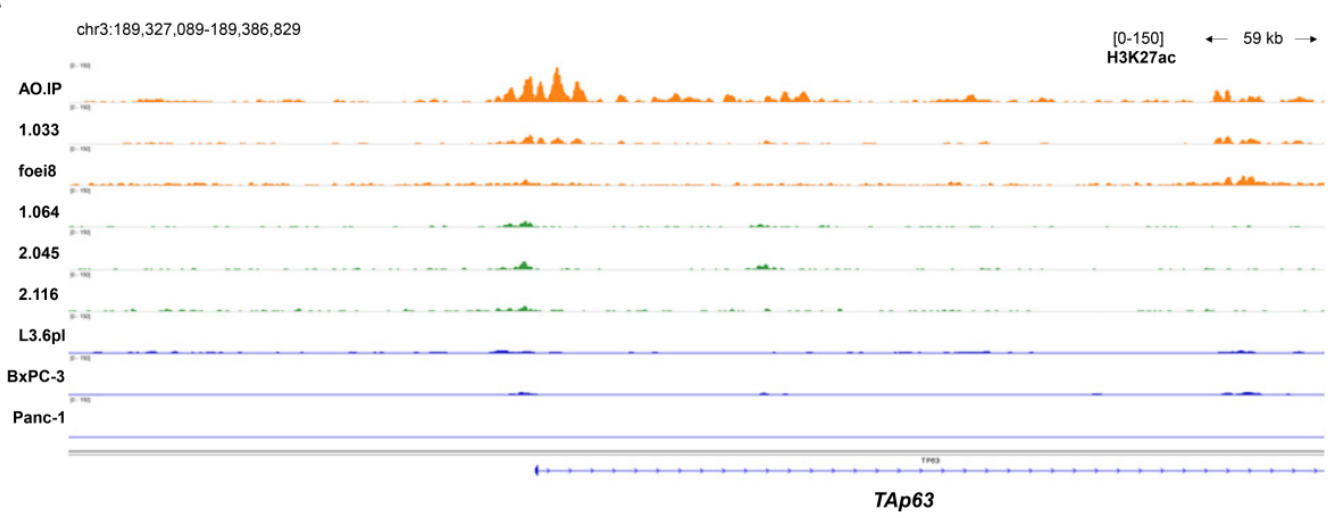

B

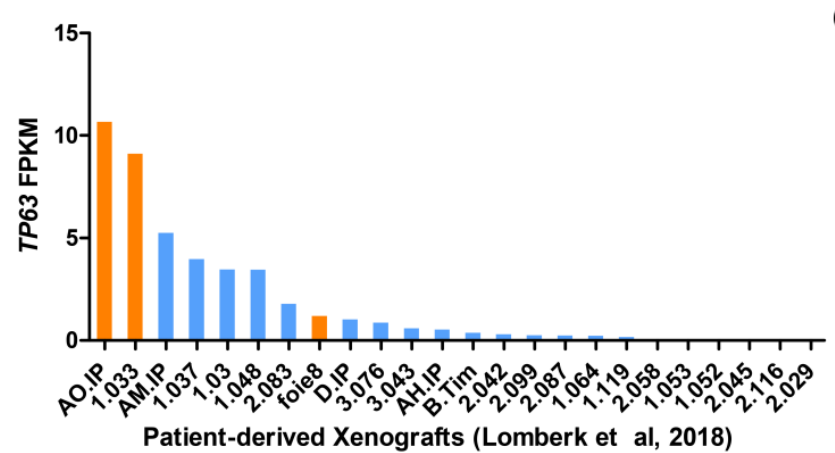

C

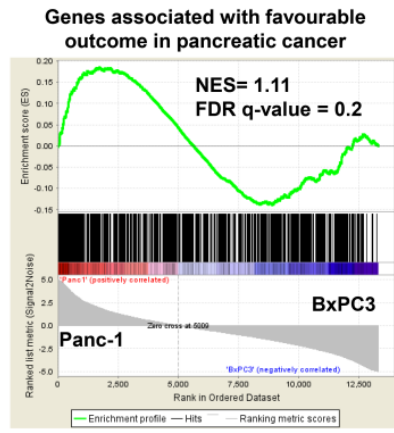

D

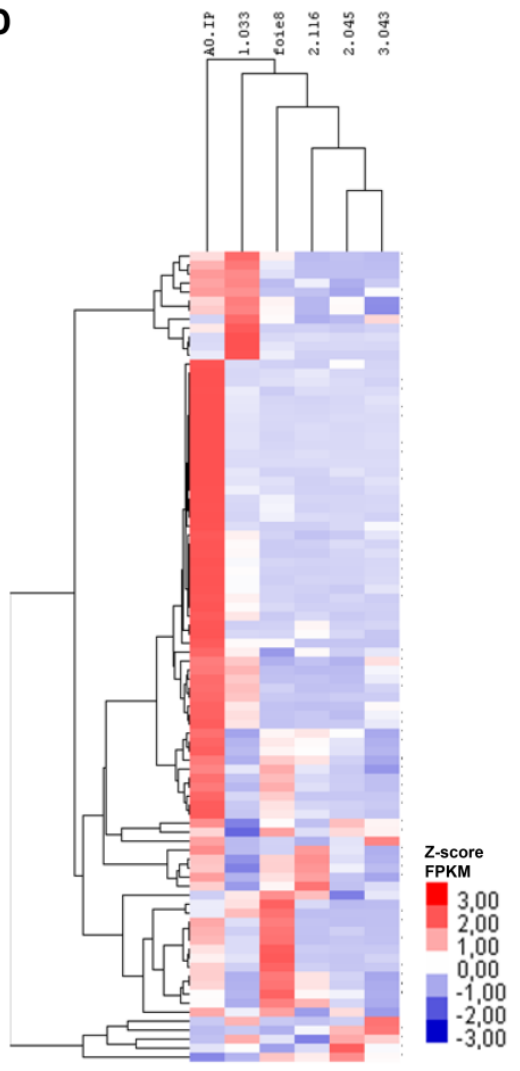

Figure 22 : (A) Occupancy profiles of H3K27ac at TSS of TAp63 for 6 xenografts, L3.6pl, BxPC3, and Panc-1 showing enrichment for only one of the xenografts. (B) Bar plot showing the FPKM values of p63 (all isoforms) calculated by CUFFLINKS/2.2.1 in the 24 PDX samples. (C) GSEA plots comparing the enrichment of genes associated with favorable outcome in pancreatic cancer in BxPC-3 compared to Panc-1 using the FPKM values of all expressed genes with the NES (normalized enrichment score) and FDR (false-discovery rate) indicated on the graph. (D) A heatmap showing the Z-scores of the FPKM values for the squamous gene signature in the 6 PDX samples shown in A. Hierarchical clusters for samples based on the expression underscores the increased expression of the signature apparent in the 3 three squamous PDX-samples. 


\subsubsection{Supplementary Figure 2 (Figure 23)}

Supplementary Figure 2

A p63н3К27ac p63non-H3K27ac

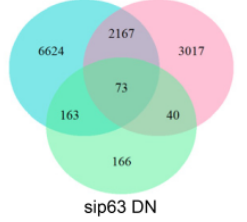

C

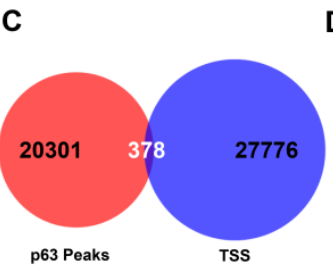

$\mathbf{F}$

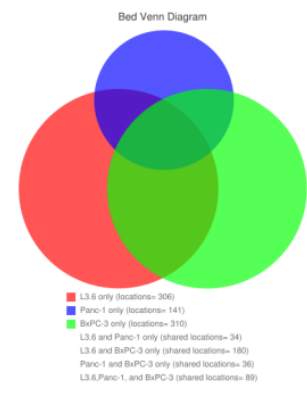

B
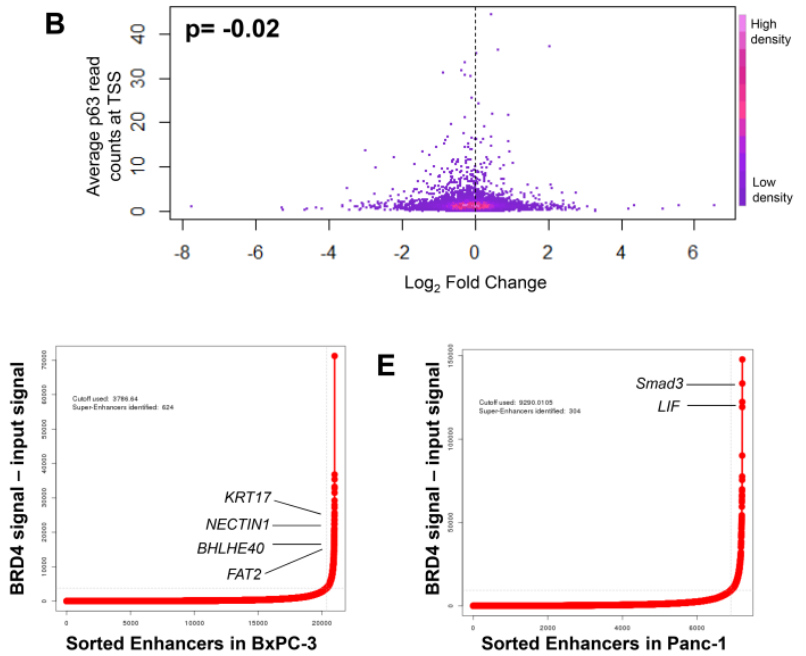

G

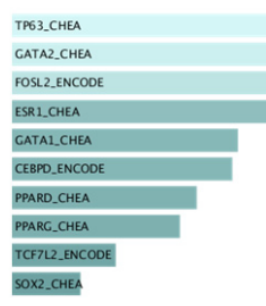

H

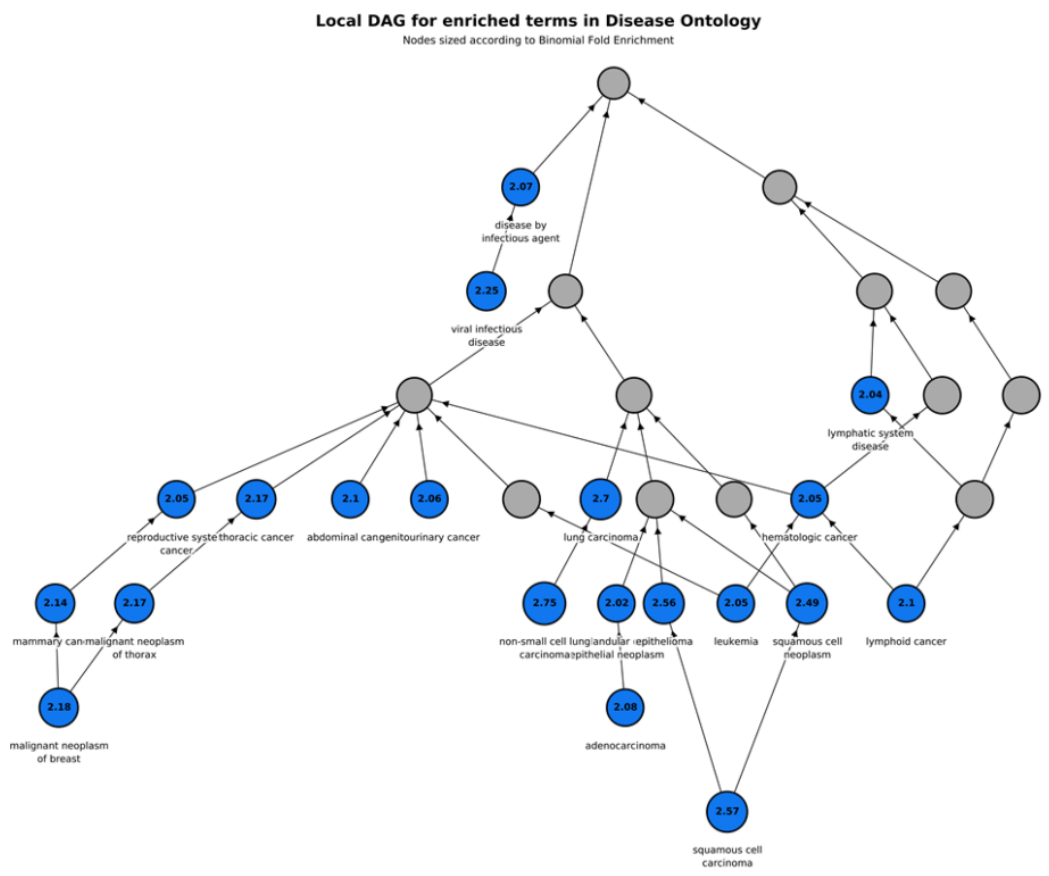


Figure 23 : (A) Venn diagram showing the overlap between down regulated genes and genes associated with p63 regions occupied with H3K27ac and regions that are not marked by H3KK27ac. Associated genes were identified using the beta-minus method using the galaxy cistrome. A slight bias for p63 dependence is shown for genes that associated with p63 peaks marked with H3K27ac. (B) Heat scatter plot showing the relationship between occupancy of p63 at TSS and the gene regulation upon knockdown of p63 showing a lack of correlation between TSS occupancy and dependence. (C) Venn Diagram showing the overlap between p63 regions and TSS in L3.6pl. (D-E) Enhancers in BxPC-3 and Panc-1 ranked based on BRD4 signal intensity using the ROSE algorithm defining 624 super enhancers. BxPC-3 shows common high ranking super enhancers with L3.6pl while Panc-1 has distinct highly ranking super enhancers. (F) Bed venn diagram depicting overlap of super enhancers in L3.6pl, BxPC-3, and Panc-1. (G) ChEA and ENCODE consensus output for super enhancer regions in L3.6pl defined by erichr. (H) Directed acyclic graph for gene ontology pathways associated with genes related to super enhancers in L3.6pl with squamous cell carcinoma as a high significant hit.

\subsubsection{Supplementary Figure 3 (Figure 24)}

A
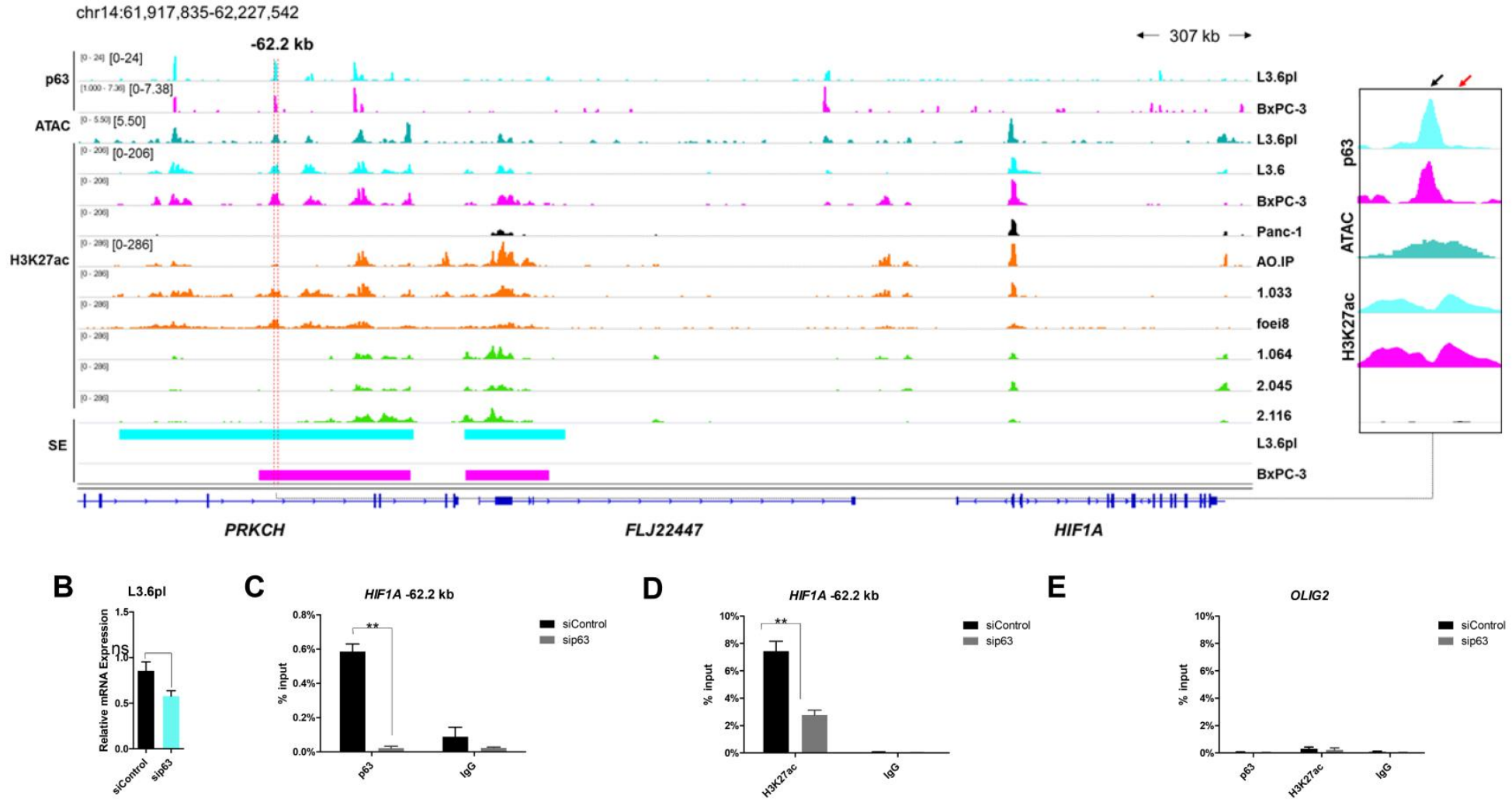

Figure 24 : (A) Occupancy profiles at HIF1A for p63 in L3.6pl and BxPC-3, ATAC seq in L3.6pl, H3K27ac in L3.6pl and BxPC-3, and 6 xenografts. (B) Gene expression analysis for HIF1A by qRT-PCR of deltaNp63 upon depletion of p63 after 48 hours shown as relative mRNA expression and normalized to the unregulated housekeeping gene (GAPDH). $n=$ 3. * P-value $<=0.05,{ }^{* *}$ P-value $<=0.01,{ }^{* * *} \mathrm{P}$-value $<=0.001$. (C-D) ChIP-qPCR analysis validating enrichment of p63 (C) and H3K27ac (D) at the highlighted region in A (-62.2 kb from TSS of HIF1A) in the control and p63 depletion after 48 hours. $n=3$ (one sample for p63 in p63 was disregarded because of decreased DNA amount to ensure significance in the decrease of the enrichment is not due to an outlier). Data are represented as mean \pm SEM. $n=2$. ${ }^{*} P$-value $<=0.05,{ }^{*} P$ value $<=0.01,{ }^{* * *} \mathrm{P}$-value $<=0.001$. (E) ChIP-qPCR analysis validating lack of enrichment of p63 and H3K27ac at a negative region (OLIG2 H3K27me3) to ensure specific signal. 


\subsubsection{Supplementary Figure 4 (Figure 25)}

A

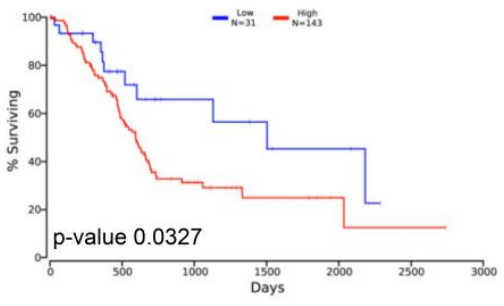

NECTIN1

C

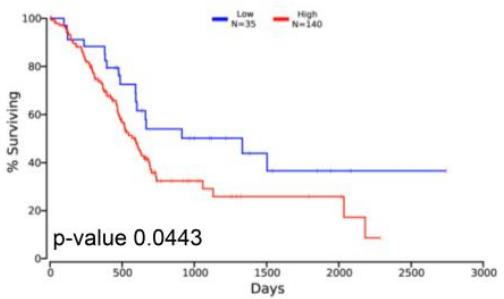

TNNI2

E

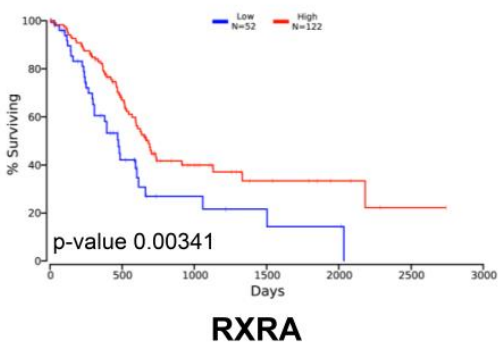

G

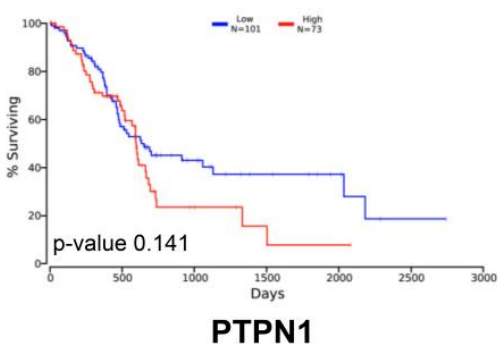

B

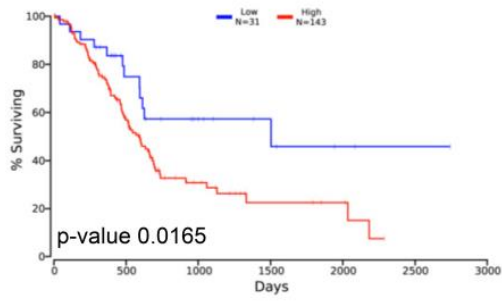

HS3ST1

D

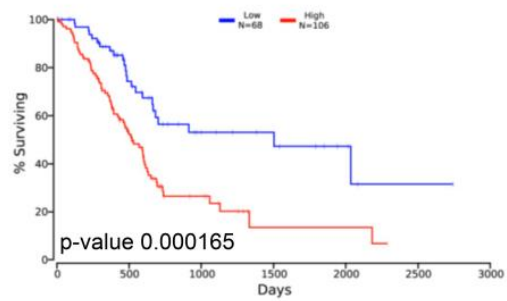

FGFBP1

$\mathbf{F}$

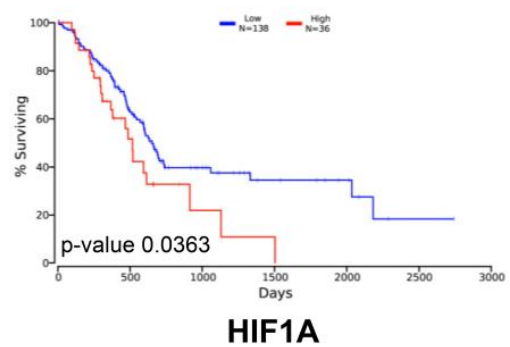

H

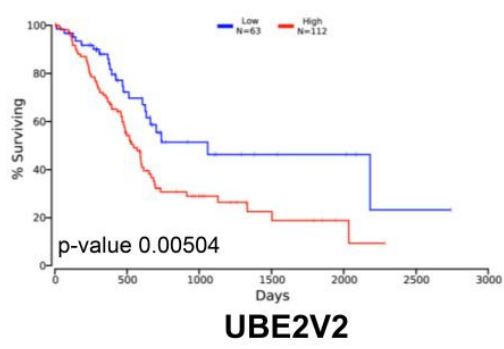

Figure $25:(\mathrm{A}-\mathrm{H})$ Kaplan-Meier plots showing the percent survival in pancreatic cancer patients (TCGA database) expressing high and low levels of the gene named on the top of each graph with p-value indicated on graph (Mantel-Cox test). 


\subsubsection{Supplementary Table S1 (Table 1) Sequences for primers used in this study} $\left(5^{\prime}-3^{\prime}\right)$

\begin{tabular}{|c|c|c|c|c|}
\hline Name & Forward Primer & Reverse Primer & Source & Application \\
\hline GAPDH & ATGGGGAAGGTGAAGGTCG & GGGGTCATTGATGGCAACAATA & Tian et al. 2013[412] & Gene Expression \\
\hline DeltaNp63 & AGAGAGAGGGACTTGAGTTCTG & GCTCACTAAATTGAGTCTGGGC & $\begin{array}{l}\text { Scheel et al. } \\
2009 \text { [413] }\end{array}$ & Gene Expression \\
\hline FAT2 & CCCACAGTGTTCACAGCTTCT & TCCAAGTCTGTGGCAGAAACC & This Study & Gene Expression \\
\hline NECTIN1 & GCGAGTTTGCTACCTTCCCT & ATTGGTGGGTTTGGCCATCA & This Study & Gene Expression \\
\hline HIF1A & TGCTTACACACAGAAATGGCCT & TACGTGAATGTGGCCTGTGC & This Study & Gene Expression \\
\hline p63_FAT2 -3.7kb & GCCTCCATGTAACTCCCAGC & CCTGTGTGTTGTTAGCCACCT & This Study & ChIP \\
\hline ac_FAT2 $-3.7 \mathrm{~kb}$ & TTCTTTCCTCCTGACTGTGCTTC & GTTGAACAGGTAGCAAGTGGTAGA & This Study & ChIP \\
\hline p63_FAT2 -46.5kb & CAGACCCTGCGTTCTGTCTT & TGATTCATGACCAGGGGTGC & This Study & ChIP \\
\hline ac_FAT2 -46.5kb & AGCTGGAAACCGACAGCTTG & GCAGTTCCATTGTCGCTGTG & This Study & ChIP \\
\hline p63_NECTIN1 $31.8 \mathrm{~kb}$ & AGGCTGGAAGGCATCTTGC & CATTGTGCAGGTGACATCGC & This Study & ChIP \\
\hline ac_NECTIN1 $31.8 \mathrm{~kb}$ & GTGCTTCCTGCTTCCCAGAAT & CCTGGTAGATAGAAGGTATTCAGCC & This Study & ChIP \\
\hline p63_NECTIN1 $36.2 \mathrm{~kb}$ & TGGGGTCTTTCCCATGCTTC & CCCAGTGACTCCTGAAACCC & This Study & ChIP \\
\hline ac_NECTIN1 $36.2 \mathrm{~kb}$ & TCCCTGGGGGAGAAAGTACAA & CACATGTGTTAACTGTTCTTGCCA & This Study & ChIP \\
\hline p63_HIF1A -62.2 kb & TACTGTGGCGGTGAAATCAACT & AGTATCTACCCTGCTCCTTGGT & This Study & ChIP \\
\hline ac_HIF1A -62.2 kb & CGGCATTTCAGCTTTGGCAG & CCCAGTGCCACAGAACAAAGA & This Study & ChIP \\
\hline OLIG2 H3K27me3 & GTCACCAACGCTCCCTGAAAT & CTGCACGCGGGTACCTATAAT & This Study & ChIP \\
\hline
\end{tabular}

\subsubsection{Supplementary Table S2 (Table 2) Accession numbers for next-generation}

\section{datasets used in this study (ChIP and ATAC-seq)}

\begin{tabular}{|c|c|c|c|c|c|}
\hline Name & Data Type & Database & Accession Number & Replicates & Source \\
\hline BxPC-3_H3K27ac & ChIP-seq & ArrayExpress & E-MTAB-7034 & 2 & This Study \\
\hline L3.6pl_H3K27ac & ChIP-seq & ArrayExpress & E-MTAB-7034 & 2 & This Study \\
\hline Panc-1_H3K27ac & ChIP-seq & ArrayExpress & E-MTAB-7034 & 2 & This Study \\
\hline AO.IP_H3K27ac & ChIP-seq & ArrayExpress & E-MTAB-5632 & 1 & Lomberk et al 2018 \\
\hline 1.033 H3K27ac & ChIP-seq & ArrayExpress & E-MTAB-5632 & 1 & Lomberk et al 2018 \\
\hline 1.03_H3K27ac & ChIP-seq & ArrayExpress & E-MTAB-5632 & 1 & Lomberk et al 2018 \\
\hline 1.048_H3K27ac & ChIP-seq & ArrayExpress & E-MTAB-5632 & 1 & Lomberk et al 2018 \\
\hline foei8 H3K27ac & ChIP-seq & ArrayExpress & E-MTAB-5632 & 1 & Lomberk et al 2018 \\
\hline 2.087_H3K27ac & ChIP-seq & ArrayExpress & E-MTAB-5632 & 1 & Lomberk et al 2018 \\
\hline $2.029 \mathrm{H} 3 \mathrm{~K} 27 \mathrm{ac}$ & ChIP-seq & ArrayExpress & E-MTAB-5632 & 1 & Lomberk et al 2018 \\
\hline 1.037_H3K27ac & ChIP-seq & ArrayExpress & E-MTAB-5632 & 1 & Lomberk et al 2018 \\
\hline AM.IP_H3K27ac & ChIP-seq & ArrayExpress & E-MTAB-5632 & 1 & Lomberk et al 2018 \\
\hline D.IP_H3K27ac & ChIP-seq & ArrayExpress & E-MTAB-5632 & 1 & Lomberk et al 2018 \\
\hline B.Tim_H3K27ac & ChIP-seq & ArrayExpress & E-MTAB-5632 & 1 & Lomberk et al 2018 \\
\hline AH.IP_H3K27ac & ChIP-seq & ArrayExpress & E-MTAB-5632 & 1 & Lomberk et al 2018 \\
\hline 3.076_H3K27ac & ChIP-seq & ArrayExpress & E-MTAB-5632 & 1 & Lomberk et al 2018 \\
\hline 2.116_H3K27ac & ChIP-seq & ArrayExpress & E-MTAB-5632 & 1 & Lomberk et al 2018 \\
\hline 2.099H3K27ac & ChIP-seq & ArrayExpress & E-MTAB-5632 & 1 & Lomberk et al 2018 \\
\hline 2.083 H3K27ac & ChIP-seq & ArrayExpress & E-MTAB-5632 & 1 & Lomberk et al 2018 \\
\hline 2.058 H3K27ac & ChIP-seq & ArrayExpress & E-MTAB-5632 & 1 & Lomberk et al 2018 \\
\hline 2.045_H3K27ac & ChIP-seq & ArrayExpress & E-MTAB-5632 & 1 & Lomberk et al 2018 \\
\hline 1.119 H3K27ac & ChIP-seq & ArrayExpress & E-MTAB-5632 & 1 & Lomberk et al 2018 \\
\hline 1.064_H3K27ac & ChIP-seq & ArrayExpress & E-MTAB-5632 & 1 & Lomberk et al 2018 \\
\hline
\end{tabular}




\begin{tabular}{|c|c|c|c|c|c|}
\hline 1.053_H3K27ac & ChIP-seq & ArrayExpress & E-MTAB-5632 & 1 & Lomberk et al 2018 \\
\hline 1.052_H3K27ac & ChIP-seq & ArrayExpress & E-MTAB-5632 & 1 & Lomberk et al 2018 \\
\hline 1.043_H3K27ac & ChIP-seq & ArrayExpress & E-MTAB-5632 & 1 & Lomberk et al 2018 \\
\hline 1.042_H3K27ac & ChIP-seq & ArrayExpress & E-MTAB-5632 & 1 & Lomberk et al 2018 \\
\hline BxPC-3_p63 & ChIP-seq & ArrayExpress & E-MTAB-7034 & 1 & This study \\
\hline L3.6pl_BRD4 & ChIP-seq & ArrayExpress & E-MTAB-7034 & 2 & This study \\
\hline BxPC-3_BRD4 & ChIP-seq & ArrayExpress & E-MTAB-7034 & 2 & This study \\
\hline L3.6pl_ATAC & ATAC-seq & ArrayExpress & E-MTAB-7035 & 2 & This study \\
\hline BxPC-3_input & ChIP-seq & ArrayExpress & E-MTAB-7034 & 1 & This study \\
\hline L3.6pl_input & ChIP-seq & ArrayExpress & E-MTAB-7034 & 2 & This study \\
\hline Panc-1_input & ChIP-seq & ArrayExpress & E-MTAB-7034 & 1 & This study \\
\hline AO.IP_input & ChIP-seq & ArrayExpress & E-MTAB-7034 & 1 & Lomberk et al 2018 \\
\hline 1.033_input & ChIP-seq & ArrayExpress & E-MTAB-5632 & 1 & Lomberk et al 2018 \\
\hline
\end{tabular}

\subsubsection{Supplementary Table S3 (Table 3) Accession numbers for next-generation}

\section{datasets used in this study (RNA-seq)}

\begin{tabular}{|c|c|c|c|c|c|}
\hline Name & Data Type & Database & Accession Number & Replicates & Source \\
\hline BxPC-3_siControl & mRNA-seq & ArrayExpress & E-MTAB-7033 & 3 & This Study \\
\hline BxPC-3 sip63 & mRNA-seq & ArrayExpress & E-MTAB-7033 & 3 & This Study \\
\hline L3.6_siControl & mRNA-seq & ArrayExpress & E-MTAB-7033 & 3 & This Study \\
\hline L3.6pl_sip63 & mRNA-seq & ArrayExpress & E-MTAB-7033 & 3 & This study \\
\hline Panc1 sicontrol & mRNA-seq & GEO & GSE90566 & 3 & Mishra et al. 2017 \\
\hline AO.IP & mRNA-seq & ArrayExpress & E-MTAB-5639 & 1 & Lomberk et al 2018 \\
\hline 1.033 & mRNA-seq & ArrayExpress & E-MTAB-5639 & 1 & Lomberk et al 2018 \\
\hline 1.03 & mRNA-seq & ArrayExpress & E-MTAB-5639 & 1 & Lomberk et al 2018 \\
\hline 1.048 & mRNA-seq & ArrayExpress & E-MTAB-5639 & 1 & Lomberk et al 2018 \\
\hline foei8 & mRNA-seq & ArrayExpress & E-MTAB-5639 & 1 & Lomberk et al 2018 \\
\hline 2.087 & mRNA-seq & ArrayExpress & E-MTAB-5639 & 1 & Lomberk et al 2018 \\
\hline 2.029 & mRNA-seq & ArrayExpress & E-MTAB-5639 & 1 & Lomberk et al 2018 \\
\hline 1.037 & mRNA-seq & ArrayExpress & E-MTAB-5639 & 1 & Lomberk et al 2018 \\
\hline AM.IP & mRNA-seq & ArrayExpress & E-MTAB-5639 & 1 & Lomberk et al 2018 \\
\hline D.IP & mRNA-seq & ArrayExpress & E-MTAB-5639 & 1 & Lomberk et al 2018 \\
\hline B.Tim & mRNA-seq & ArrayExpress & E-MTAB-5639 & 1 & Lomberk et al 2018 \\
\hline AH.IP & mRNA-seq & ArrayExpress & E-MTAB-5639 & 1 & Lomberk et al 2018 \\
\hline 3.076 & mRNA-seq & ArrayExpress & E-MTAB-5639 & 1 & Lomberk et al 2018 \\
\hline 2.116 & mRNA-seq & ArrayExpress & E-MTAB-5639 & 1 & Lomberk et al 2018 \\
\hline 2.099 & mRNA-seq & ArrayExpress & E-MTAB-5639 & 1 & Lomberk et al 2018 \\
\hline 2.083 & mRNA-seq & ArrayExpress & E-MTAB-5639 & 1 & Lomberk et al 2018 \\
\hline 2.058 & mRNA-seq & ArrayExpress & E-MTAB-5639 & 1 & Lomberk et al 2018 \\
\hline 2.045 & mRNA-seq & ArrayExpress & E-MTAB-5639 & 1 & Lomberk et al 2018 \\
\hline 1.119 & mRNA-seq & ArrayExpress & E-MTAB-5639 & 1 & Lomberk et al 2018 \\
\hline 1.064 & mRNA-seq & ArrayExpress & E-MTAB-5639 & 1 & Lomberk et al 2018 \\
\hline 1.053 & mRNA-seq & ArrayExpress & E-MTAB-5639 & 1 & Lomberk et al 2018 \\
\hline 1.052 & mRNA-seq & ArrayExpress & E-MTAB-5639 & 1 & Lomberk et al 2018 \\
\hline 1.043 & mRNA-seq & ArrayExpress & E-MTAB-5639 & 1 & Lomberk et al 2018 \\
\hline
\end{tabular}




\subsubsection{Supplementary Tables (S4-S7) provided in Excel Format (online)}

Supplementary Table S4: H3K27ac gained regions in L3.6pl and BxPC-3

Supplementary Table S5: Squamous subtype-specific super enhancers

Supplementary Table S6: GSEA table for enrichment of C2 pathways in siControl compared to sip63 in L3.6pl

Supplementary Table S7: GSEA table for enrichment of C2 pathways in sip63 compared to siControl in L3.6pl 


\section{Manuscript II}

\section{CHAPTER 5}

\section{The Role of BET Inhibition in Chemoresistance and Resensitization in Pancreatic Ductal Adenocarcinoma}

Feda H. Hamdan ${ }^{1}$, Ana P. Kutschat ${ }^{1}$, and Steven A. Johnsen ${ }^{1, *}$

1 Section of Tumor Epigenetics, Department of General, Visceral and Pediatric Surgery, University Medical Center Göttingen, 37075 Göttingen, Germany

* To whom correspondence should be addressed. Tel: +49 55139 13711; Fax: +49 55139 12297; Email: steven.johnsen@med.uni-goettingen.de

\section{Keywords}

Pancreatic cancer, Paclitaxel, BET inhibitors, JQ1, chemo-resistance, chemo-sensitization

Contributions: Figure $26 \mathrm{G}$ and $27 \mathrm{~B}$ were performed by A.P.K as part of a master rotation under the supervision of F.H.H. All the other figures and analysis was done by F.H.H. F.H.H. and S.A.J. conceived the ideas for this project and wrote the manuscript. 


\subsection{Abstract}

Resistance to conventional chemotherapy is one of the main causes of dismal survival rates in pancreatic cancer patients. Recent findings showed that inhibition of the Bromodomain and Extraterminal (BET) protein family is effective, both alone and in combination with conventional chemotherapy, in decreasing pancreatic tumor growth in patient-derived xenografts. Thus, we aim to evaluate the potential role of BET inhibitors as an adjuvant therapeutic option in pancreatic cancer.

We established L3.6pl pancreatic cells that are resistant to paclitaxel by maintaining them in incrementally higher concentrations for 3 months. Paclitaxel resistant cells showed a half maximal inhibitory concentration (IC50) which is 100 fold more than that of parental cells. Intriguingly, we report that low concentrations of the BET inhibitor, JQ1, not only sensitized cells to paclitaxel, but also induced significant re-sensitization of chemoresistant cells. In order to elucidate the mechanism by which BET inhibition induces chemo-sensitization, we investigated the differential gene expression profiles of resistant and sensitive cells. Thereby, we uncovered that $\mathrm{BET}$ inhibition reverses the regulation of transcriptionally-activated programs in resistant cells which are associated with inflammation and epithelial-to-mesenchymal transition. Interestingly, these genes showed a major tendency to gain BRD4 at putative enhancer regions. Furthermore, we observed that BET-dependent super enhancers that are specific to resistant cells are associated with genes that are correlated to poor prognosis in pancreatic cancer. Finally, we identified different regulation patterns for BET-dependent genes when subjected to low and high doses of the BET inhibitor JQ1.

In conclusion, we provide evidence that BET inhibitors can potentially be used as adjuvant agents in pancreatic cancer. However, such indication is not due to their anti-proliferative effects 
that require higher concentrations and possibly lead to intolerable adverse effects, but rather to their transcriptional regulatory functions that attenuate the activated programs in chemoresistant cancers.

\subsection{Introduction}

In spite of relatively lower incidence rates compared to more common malignancies, pancreatic cancer emerges as one of the leading causes of cancer-related deaths around the world [153, 414]. In the upcoming 10 years, pancreatic cancer is predicted to exceed breast and colorectal cancer and become the second most common cause of cancer-related mortalities [415]. Resistance to conventional chemotherapy is one of the main causes of dismal survival rates in pancreatic cancer patients [246]. Consequently, there is an urgent need to establish and test new unconventional treatments that can efficiently improve the prognosis of pancreatic cancer.

Interestingly, inhibition of the epigenetic reader family of Bromodomain and Extraterminal (BET) proteins was reported to be effective in decreasing pancreatic tumor growth in patient-derived xenografts when administered alone or in combination with conventional chemotherapies [198, 209]. BRD4, the most thoroughly investigated member of the BET family, plays an important role in cell growth, cell cycle control, and gene transcription [416]. BRD4 knockout is lethal in mouse embryos which suggests that BRD4 has a crucial role in fundamental cellular processes [417]. However, upon inhibition of BRD4 with small molecular inhibitors, only a specific subset of genes is affected, particularly cancer-promoting genes such as the MYC proto-oncogene and the apoptotic regulator B Cell Lymphoma 2 (BCL2) $[205,286]$.

JQ1, the most studied BET inhibitor, was reported to show efficacy in chemo-resistant pancreatic cancer cells in three-dimensional collagen [210]. Moreover, general drug screening found that JQ1 and paclitaxel have a synergistic effect in triple negative breast cancer [418]. 
Paclitaxel, along with gemcitabine, is one of the first line conventional therapies that are commonly used in the management of pancreatic cancer [172]. Paclitaxel binds to microtubules forming the mitotic spindle and enhances its polymerization, increases its stability, and prevents its disassembly leading the cells into an arrested cell cycle state and apoptosis [182, 419].

Paclitaxel treatment was reported to induce a pro-inflammatory state in patients [420]. In contrast to other chemotherapeutic agents like doxorubicin and vinblastine, non-steroidal antiinflammatory drugs (NSAIDs) did not show synergy with paclitaxel [421]. Interestingly, paclitaxel- resistant ovarian cancer cells showed a transcriptional activation of inflammatory response $[422,423]$ and stimulation of a pro-inflammatory environment lead to the development of resistance to paclitaxel [424]. Furthermore, inhibition of the Nuclear Factor kappa-light-chainenhancer of activated B cells (NF-KB) pro-inflammatory signaling sensitized breast cancer cells to paclitaxel [425]. Moreover, inhibition of NF-KB signaling activation by a tumor necrosis factor alpha (TNFa) inhibitor sensitized pancreatic cancer cells to paclitaxel [426].

Notably, resistance to conventional therapy was linked to activation of program driving superenhancers in leukemia [427]. Super enhancers have drawn significant attention in cancer research due to their high dependence on targetable factors such as BRD4 and their ability to activate specific oncogenic programs $[123,124]$. They include large stretches of activated regions that are bound by a plethora of transcription factors [248]. To date, the mechanism of dependence of these enhancers on BET inhibition has not been fully elucidated. Studies investigating the efficacy of BET inhibitors usually use high concentrations that are just below the maximum-tolerated dose (MTD) [428-431]. So far, the relation between dose and enhancer deactivation has not been established. However, it is usually reported that different cell lines have various sensitivities toward BET inhibitors [212, 315, 432]. Accordingly, further studies are 
necessary to uncover the mechanisms of super enhancer sensitivity toward BET inhibition to guide pre-clinical and clinical studies to use the most effective dosage.

In this study, we investigated the potential use of BET inhibition as sensitizers to chemotherapeutic agents, in particular paclitaxel, in the highly metastatic pancreatic cancer cell line L3.6pl. We observed that low concentrations of JQ1, which do not affect the proliferation to a high extent on its own, sensitize normal and highly resistant cells to paclitaxel. Moreover, we report that paclitaxel-resistant cells exhibit transcriptional activation of inflammatory signaling that is reversed by treatment with JQ1. Interestingly, we observed that the transcriptional changes in paclitaxel resistance indicated a change to a more migratory phenotype and a molecular switch that were reversed by JQ1. Additionally, we uncovered that the mechanism of this activation is mediated by a gain of BRD4 on putative enhancers and super enhancers which drive genes that correlate with worse prognosis in pancreatic cancer patients. Interestingly, we also report that a certain cluster of genes regulated by JQ1 can be preferentially affected by low or high concentrations of JQ1 in a manner related to the occupancy of BRD4 at the TSS of these genes. Altogether, we provide a rationale to use BET inhibitors in low concentrations along with paclitaxel to enhance its effects and de-activate programs that are related with poorer prognosis in pancreatic cancer patients. 


\subsection{Results}

\subsubsection{BET inhibitors sensitize normal and resistant pancreatic cancer cells to Paclitaxel}

Sensitivity to paclitaxel and the BET inhibitor, JQ1, was evaluated in the highly metastatic pancreatic cancer cell line, L3.6pl, by proliferation assays using a series of different concentrations. While the half maximal inhibitory concentration (IC50) of paclitaxel was defined as $2 \mathrm{nM}, 316 \mathrm{nM}$ were needed from the epigenetic inhibitor, JQ1, to hinder the growth of $50 \%$ of the cells (Figure 26A, 26B). In order to check if JQ1 can sensitize L3.6pl cells to paclitaxel, we combined different concentrations with the JQ1 concentration required to inhibit 10\% (IC10) and $25 \%$ (IC25) of the cells (58 nM and $133 \mathrm{nM}$ ), respectively. Interestingly, cells showed increased sensitivity to paclitaxel even when combined with very low concentrations of JQ1 leading the IC50 of paclitaxel to drop to $0.81 \mathrm{nM}$ and $0.39 \mathrm{nM}$ with increased concentrations of JQ1 (Figure 26C, 26D).

To check if sensitization to paclitaxel by JQ1 can occur after resistance is already present, we established a paclitaxel-resistant cell line by maintaining the cells in increasing concentrations of paclitaxel (scheme in Figure 26E). We started with the IC50 of paclitaxel in L3.6pl and continued doubling the concentration till the cells were thriving in $64 \mathrm{nM}$ of paclitaxel and exhibiting an IC50 that is 100 times of the normal cells (Figure 26F). Remarkably, the established resistant cells were sensitized to paclitaxel upon co-treatment with the IC25 of JQ1 (Figure 26G). 


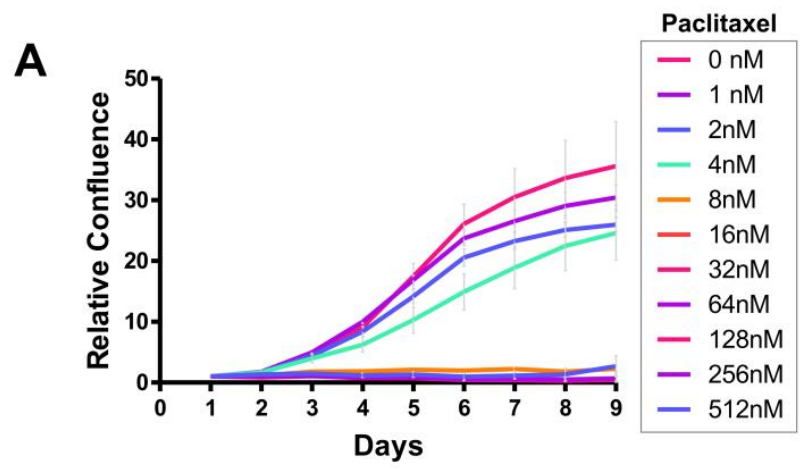

B

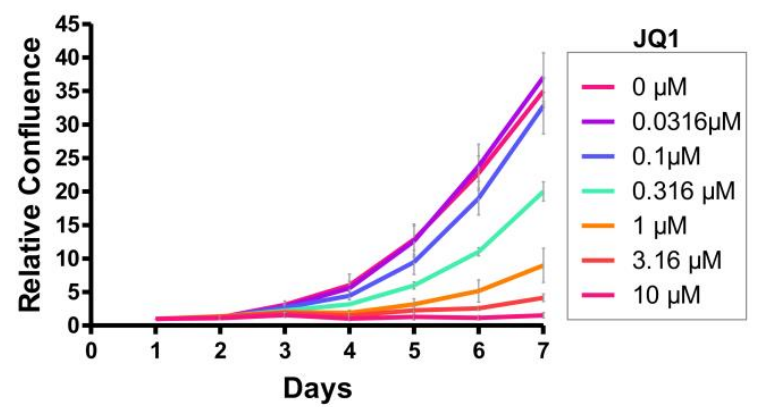

C

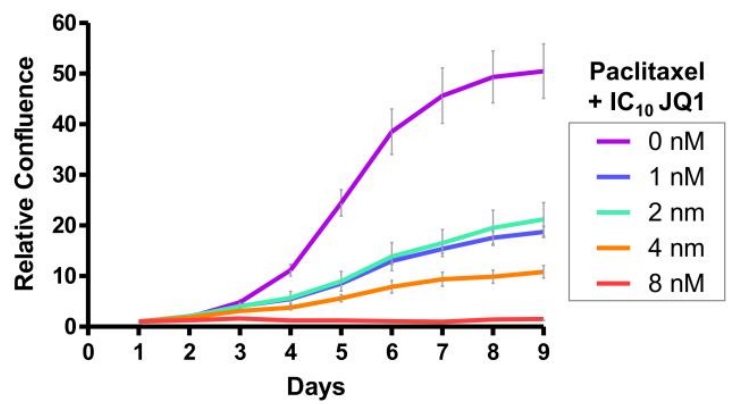

D

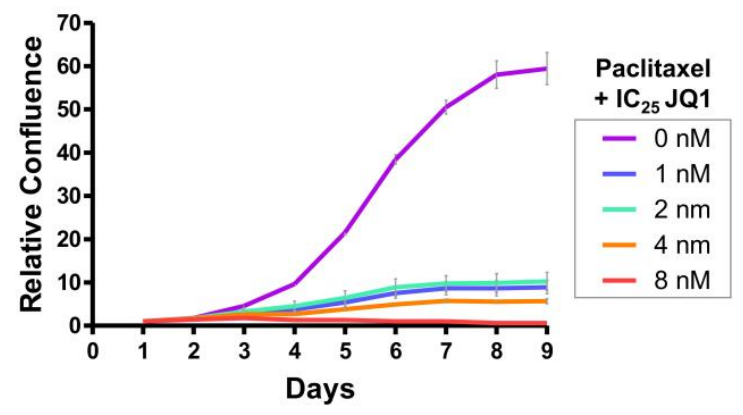

E
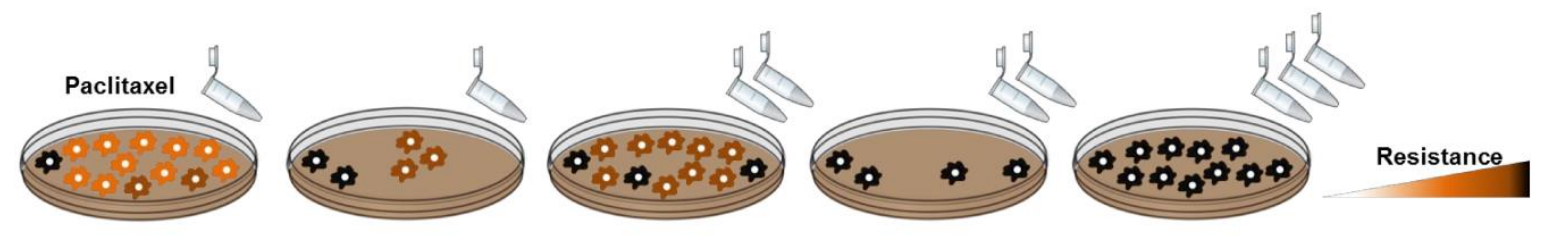

$\mathbf{F}$

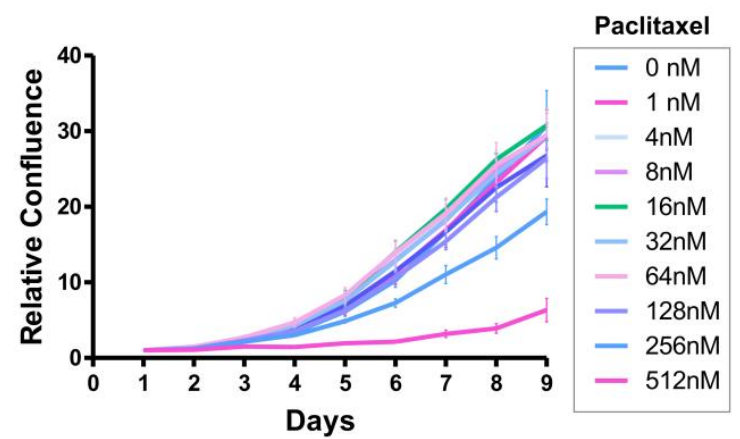

G L3.6 resistant cells (PacR)

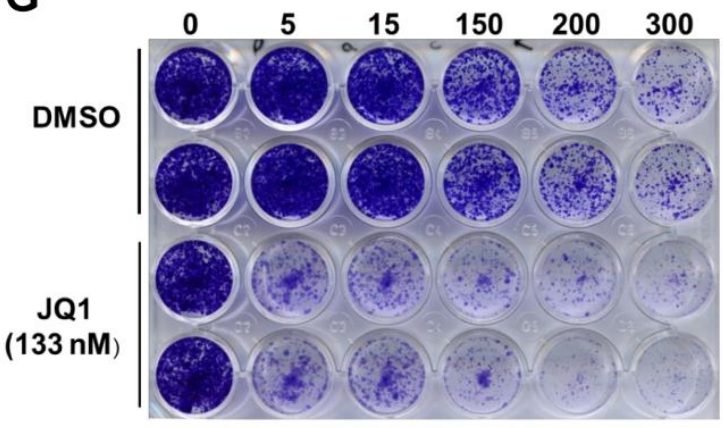

Figure 26 : JQ1 sensitizes L3.6pl cells to paclitaxel. Growth curves for L3.6pl upon treatment with (A) paclitaxel, (B) JQ1, (C) paclitaxel and IC10 of JQ1, (D) paclitaxel and IC25 of JQ1. y- axis: relative confluence normalized to day $1, \mathrm{x}$-axis; day of treatment. 1000 cells were seeded in 96-well plate and treated every two days. (E) Schematic approach for establishment of L3.6pl cells resistant to paclitaxel by maintaining them in incremental concentrations (F) Growth curve of resistant cells show their toleration to high paclitaxel concentrations (G) Crystal violet showing the ability of paclitaxel resistant cells to survive at much higher concentrations of paclitaxel debilitated by low concentrations of JQ1. 5000 cells were seeded in a 24-well plate and treated every two days up to the eighth day when cells were stained. 


\subsubsection{JQ1 reverses the regulation of transcriptionally-activated genes in resistant cells}

To understand the mechanisms by which JQ1 leads to paclitaxel sensitization, we studied the gene transcription profile changes in paclitaxel-resistant (PacR) cells compared to normal L3.6pl cells and upon treatment with $500 \mathrm{nM}$ of JQ1. RNA sequencing (RNA-seq) uncovered the activation of certain clusters of genes in PacR cells which are reversed by the treatment with JQ1 (Figure 27A). These genes include, for example, Apolipoprotein B mRNA Editing Enzyme Catalytic Subunit $3 C(A P O B E C 3 C)$ which plays a role in the induction of mutations and meiotic recombination [433, 434]. Additionally, these clusters include genes related to epithelial-tomesenchymal transition (EMT) programs which correlate with higher invasion and metastasis rates in various cancers such as Semaphorin 3C (SEMA3C), Caveolin 1 (CAV1), and Cadherin 5 (CDH5) [435-438]. Interestingly, inflammation-related genes, for example interleukin 6 family cytokine (LIF) and TNF Alpha Induced Protein 3 (TNFAIP3), were also found in BET-responsive clusters. These patterns which were observed in gene set enrichment analysis (GSEA) were validated by quantitative real-time PCR (Figure 27B - 27D). Particularly, GSEA analysis uncovered a major activation of TNF- alpha signaling that is reversed by JQ1 treatment. Moreover, a molecular subtype switch and migration was equally reversed upon JQ1 treatment. In summary, we have observed the transcriptional activation of various programs including inflammation and migration in paclitaxel-resistant cells which can be reversed by BET inhibition. 


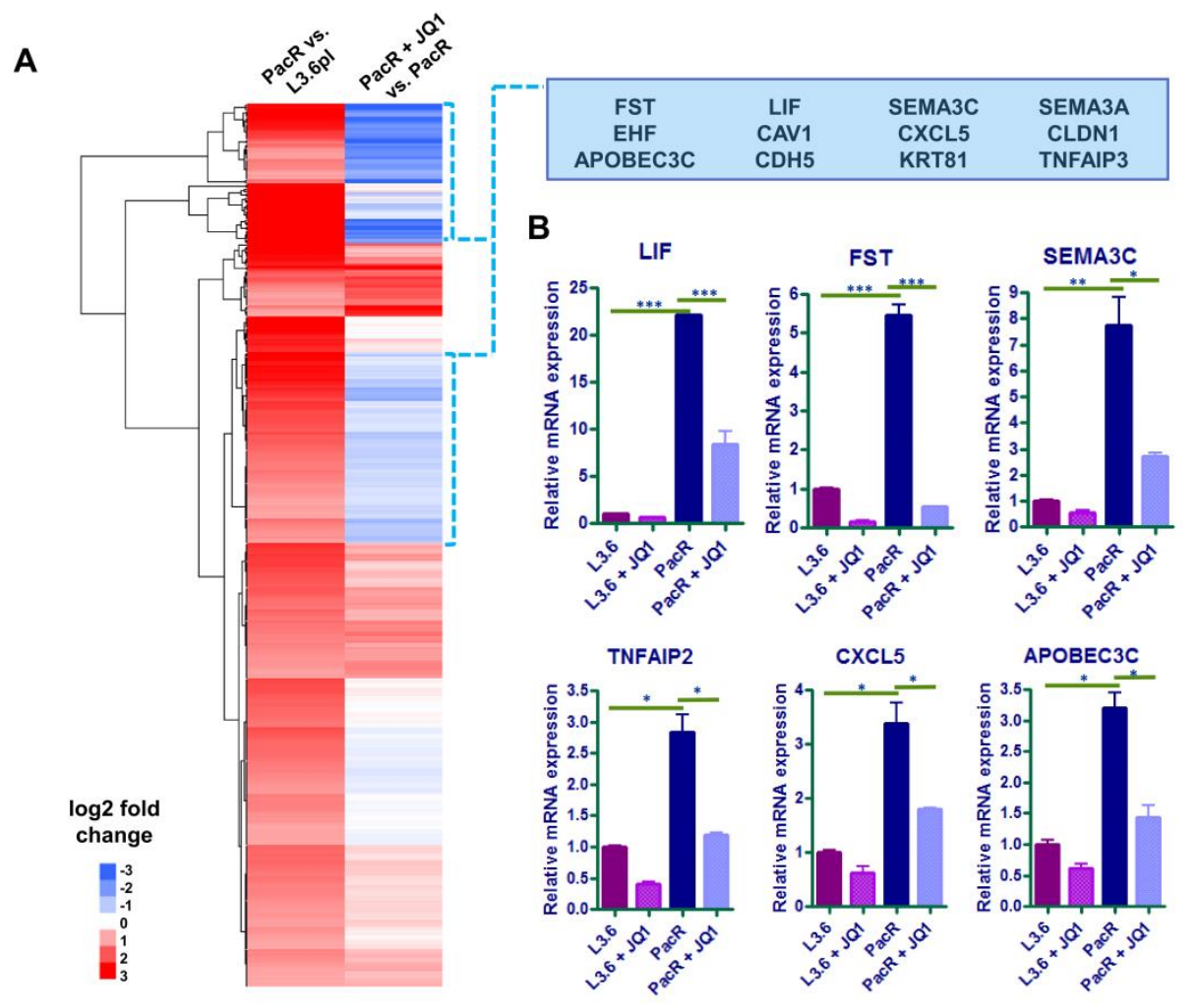

C
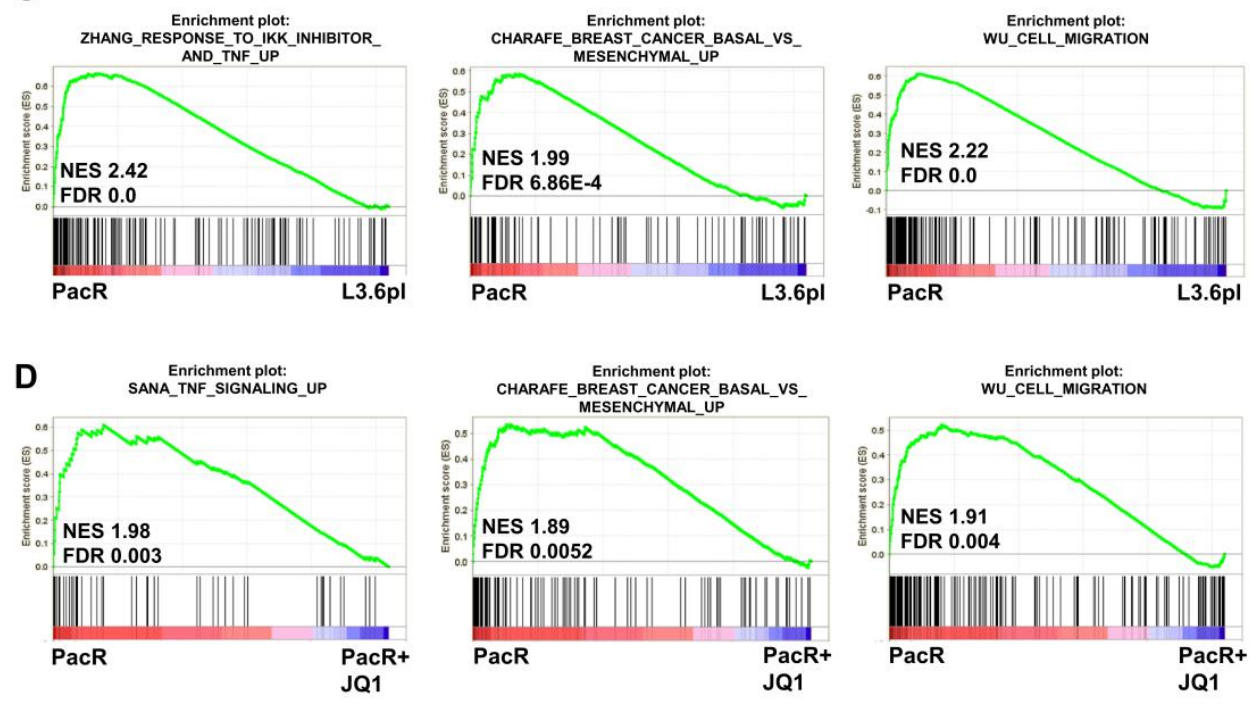

Figure 27 : JQ1 reverses transcriptionally activated programs in resistant cells. (A) Heatmap showing the significantly upregulated and downregulated genes (2 folds) in paclitaxel resistant cells compared to sensitive cells with the corresponding regulation upon treatment with JQ1. Clusters of genes show reversed regulation upon JQ1 treatment. (B) Validation of genes that are upregulated in paclitaxel resistant cells and downregulated with JQ1 treatment. Such genes include major activators in inflammatory pathways such as NFK $\beta$ and TNFa. (C, D) GSEA analysis showing significantly enriched pathways in PacR compared to sensitive cells (C) and compared to PacR cells treated with JQ1 treatment (D). 


\subsubsection{BRD4 is gained in putative enhancer regions of transcriptionally-activated genes in resistant cells}

To uncover the mechanisms by which JQ1 reverses transcriptionally-activated programs in resistant cells, we performed chromatin immunoprecipitation followed by high throughput sequencing (ChIP-seq) for BRD4 in PacR and L3.6pl cells. Thereby, we identified 1165 regions that gained BRD4 occupancy in PacR cells using differential binding analysis (Figure 28A). Notably, only 4 regions significantly lost BRD4 occupancy and the majority of the BRD4 gained regions were localized at putative enhancers as the majority did not intersect with known transcription start sites (TSS) (Figure 28B). Genes associated with those regions included genes that we identified as sensitive to JQ1 treatment and upregulated in PacR cells using RNA-seq (Figure 28C). Interestingly, we observed that BRD4 gained regions in resistant cells comprised open chromatin regions identified by Assay for Transposase-Accessible Chromatin (ATAC-seq) in normal cells (Figure 28C). Motif analysis revealed the significant enrichment for binding sequences for the transcription factors ETS Homologous Factor (EHF) and Forkhead Box A1 (FOXA1) (Figure 28D). FOXA1 was reported to play a significant role in activation of enhancers associated with metastasis in pancreatic cancer while EHF is part of the ETS family which shows high dependence on BET inhibition (Figure 28E) [154, 439]. Furthermore, pathway enrichment analysis from the molecular signatures database (MSigDB) identified mitotic phase and cell cycle as enriched signatures in genes associated with the BRD4 gained regions in PacR cells (Figure 28F). Additionally, Encyclopedia of DNA Elements (ENCODE) and ChIP Enrichment Analysis (ChEA) showed the known BRD4 target, MYC Proto-Oncogene (MYC), as a highly enriched transcription factor in these regions (Figure 28G). Altogether, we uncovered that the transcriptional activation of certain programs in resistant cells is associated with a gain of BRD4 at putative enhancer regions hence their sensitivity to BET inhibition. 
A

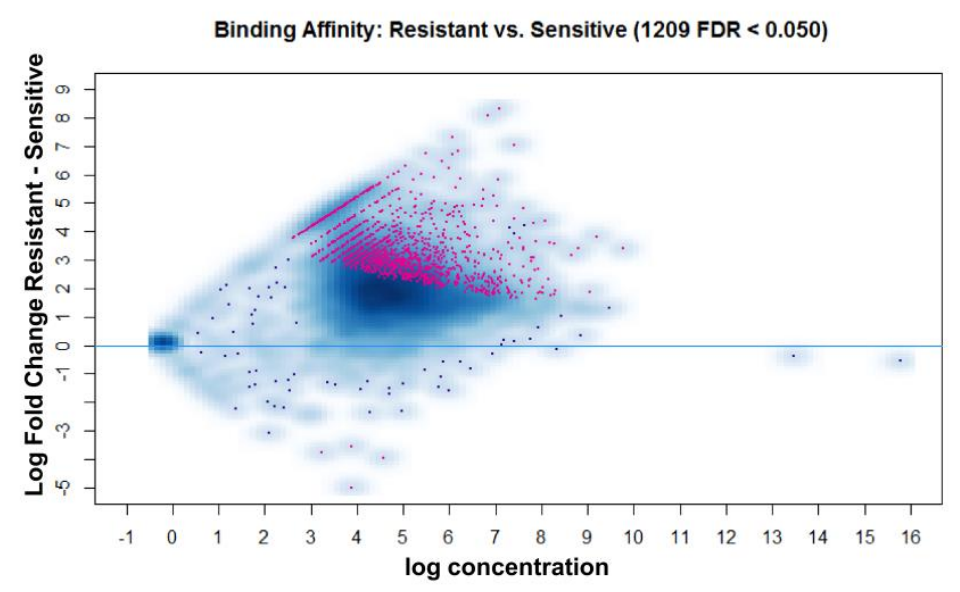

C

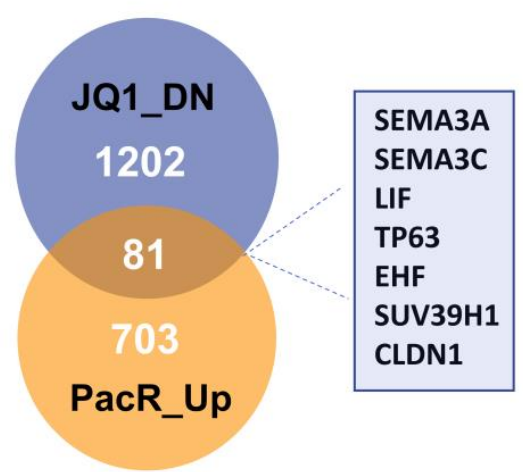

$\mathbf{F}$

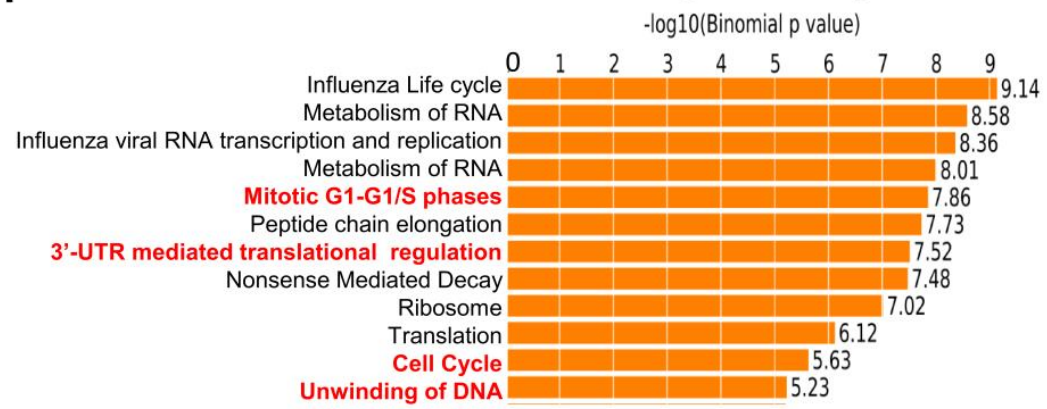

B

1165 regions ( > 2 Fold Change) TSS

(225)

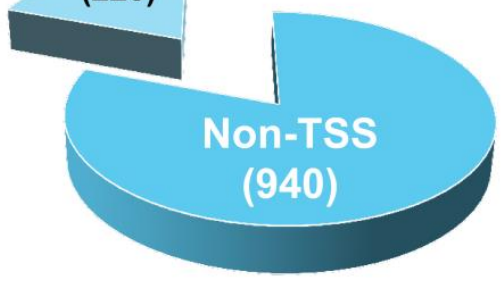

E

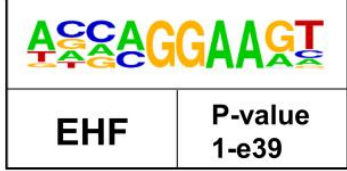

PAAGTAAACA

Figure 28 : BRD4 is gained at putative enhancers near transcriptionally active genes in resistant cells. (A) Binding affinity plot showing differentially bound regions of BRD4 in PacR cells compared to sensitive cells. (B) Pie chart showing that around $81 \%$ of the regions are not associated with TSS. (C) Venn diagram showing overlap between genes associated with gained regions and downregulated genes by JQ1. (D) Occupancy profiles of BRD4 for gained regions in PacR cells and co-localization with already open regions in sensitive cells identified by ATAC-seq. (E) Enriched motifs found by HOMER in BRD4-gained regions. (F) GREAT analysis gained regions uncovering association with paclitaxel-related pathways (shown in red). (G) ChEA and ENCODE consensus output for gained regions defined by erichr. 


\subsubsection{Gained super enhancers in resistant cells drive programs leading to poorer prognosis}

Given the role of BRD4 at enhancers in resistant cells and the well-established implication of BRD4 in super enhancer dependence and activation, we investigated the differential epigenetic gene activation in resistant and normal cells. This was done by studying their acetylation of Histone 3 Lysine 27 (H3K27ac) profiles and identified super enhancers (Figure 29A). Notably, certain genes that were upregulated in PacR cells and showed sensitivity to BET inhibition , such as Semaphorin 3A (SEMA3A), SEMA3C, LIF, and EHF, were found to be associated with super enhancers. The change in H3K27ac and BRD4 profiles in PacR cells led to identification of 104 PacR-specific super enhancers (SEs) that are not identified as SEs in normal cells (Figure 29B). Of the genes associated with PacR-specific SEs, we uncovered 8 genes that are also upregulated in PacR cells and downregulated upon treatment with JQ1 (Figure 29C). Interestingly, high expression of these genes correlated with a poorer prognosis in pancreatic cancer patients as seen in data from the Cancer Genome Atlas (TCGA) with SEMA3C, and Ras Homolog Family Member V (RHOV) shown as example (Figure 29D, 29E). For these genes, a gain of BRD4 and H3K27ac marked in some cases the activation of multiple super enhancers near one gene as in the case of LIF (Figure 29F). These gained peaks were localized at regions of open chromatin in normal cells and showed mild occupancy of JQ1 at these regions observed by Chem-seq. In other cases, gain of BRD4 and H3K27ac peaks was observed at regions devoid of open regions in normal cells and showing low occupancy of JQ1 (Figure 29G). This indicates that some of the activated regions upon resistance were already mildly active in parental cells. However, another group of regions were completely inactive in parental cells and only activated upon resistance. 
A

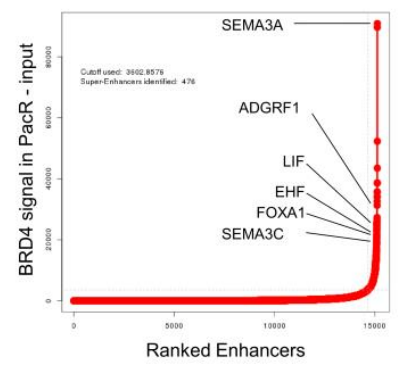

C

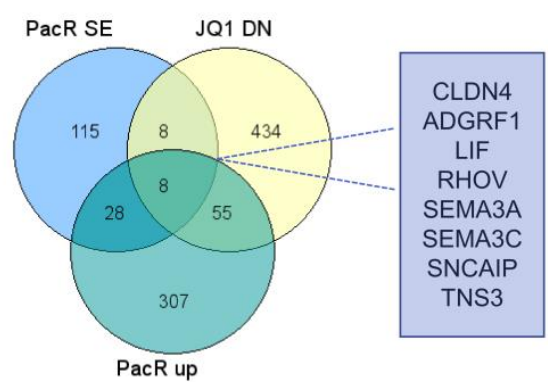

D
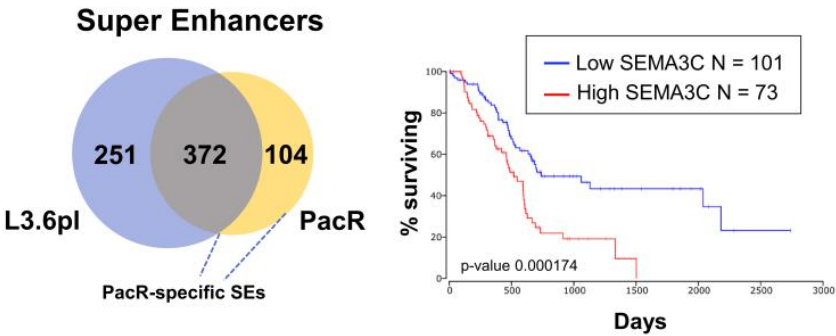

E

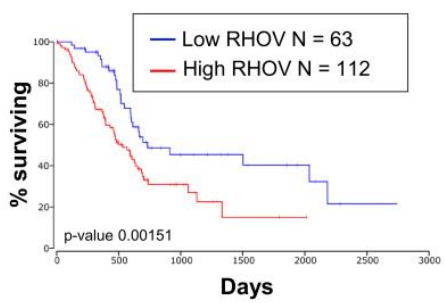

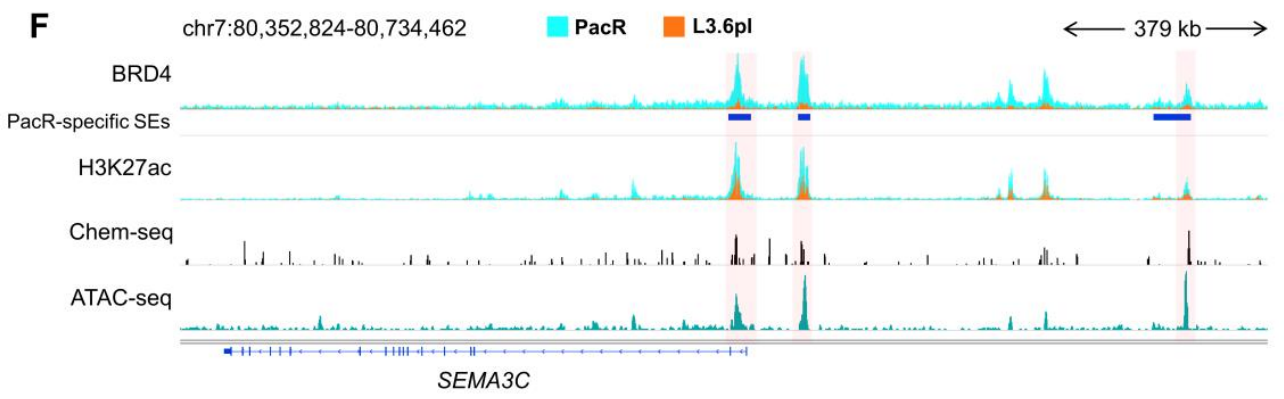

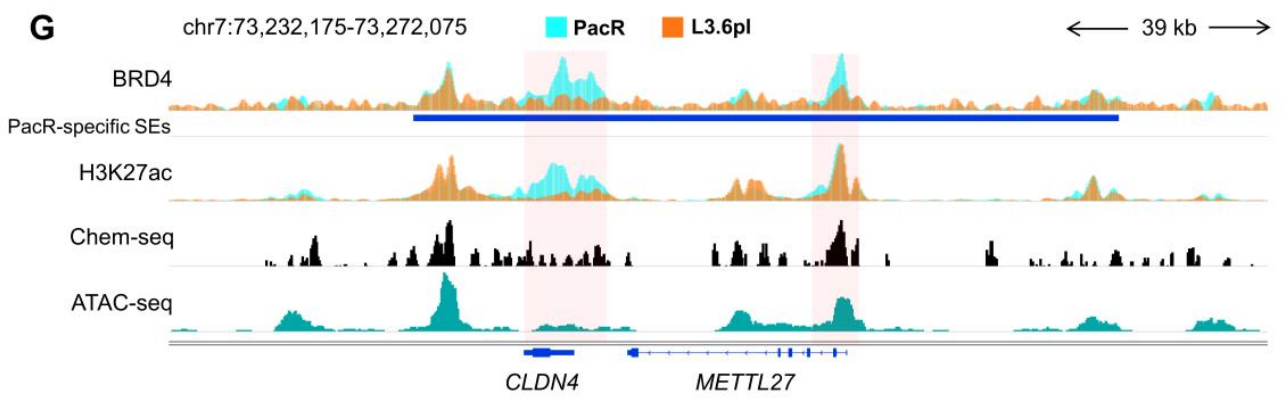

Figure 29 : BET dependent super enhancers are activated in resistant cells. (A) ROSE algorithm output showing the identified super enhancers in resistant cells with the y-axis corresponding to the intensity of BRD4 signal and the $\mathrm{x}$-axis to the rank of enhancers. Genes of interest associated with the ranked super enhancer are shown as examples. (B) Venn diagram showing the overlap between super enhancers identified in PacR and sensitive cells. (C) Venn diagram showing the genes that are associated with PacR-specific super enhancers, upregulated in resistant cells and sensitive to JQ1. (D, E) Kaplan-Meier plot for pancreatic cancer patients from the TCGA database with high and low expression of SEMA3C and RHOV. ( $F, G)$ Occupancy profiles of BRD4 and H3K27ac at LIF and CLDN4 in PacR and sensitive cells accompanied by ATAC-seq and Chem-seq profiles. PacR-specific super enhancers are shown as blue boxes. 


\subsubsection{BET-dependent genes respond differentially to low and high doses of JQ1}

Given the ability of low concentrations of JQ1 to sensitize cells to paclitaxel, we investigated the effects of JQ1 on gene transcription upon treatment for 24 hours with $100 \mathrm{nM}$ and $500 \mathrm{nM}$ JQ1. We have already shown that $100 \mathrm{nM}$ inhibits the growth of approximately $20 \%$ of cells while 500 $\mathrm{nM}$ affects around $70 \%$ of cell growth (Figure 26B). Upon studying the gene transcriptional changes of regulated genes at the two concentrations, we identified 16 patterns of gene transcriptional modulation. Notably, the most significant cluster of genes included 222 genes which were downregulated at $100 \mathrm{nM}$ and further downregulated with $500 \mathrm{nM}$. Most interestingly, other genes showed preference in their response to one condition where they were downregulated more profoundly by $100 \mathrm{nM}$ and others by $500 \mathrm{nM}$ (Figure 30A, 30B, 30D). Remarkably, a cluster comprised of 45 histone genes were shown to be upregulated in the case of high concentrations of JQ1 (Figure 30C). This is probably due to perturbed processing of the poly adenylated histone mRNAs as BET inhibition decreases the recruitment of CDK9 which was shown to play a significant role in $3^{\prime}$ end processing of replication-dependent histones [201, 440-442].

Notably, the cluster of genes which responded more to $100 \mathrm{nM}$ showed significantly lower occupation of BRD4 at their TSS compared to the clusters which showed no preference or were more affected by $500 \mathrm{nM}$ JQ1 (Figure 30E). Gene ontology for genes responsive to lower concentrations showed that already at lower concentration, JQ1 can attenuate inflammation and regulation of gene cycle explaining the ability of lower concentration to sensitize cells to paclitaxel (Figure 30F, 30G). 
A

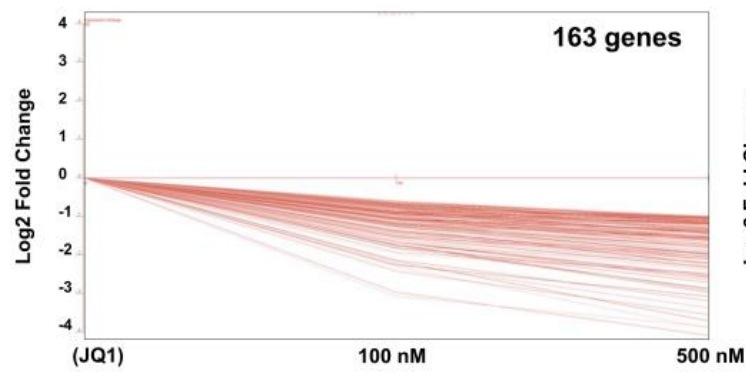

C

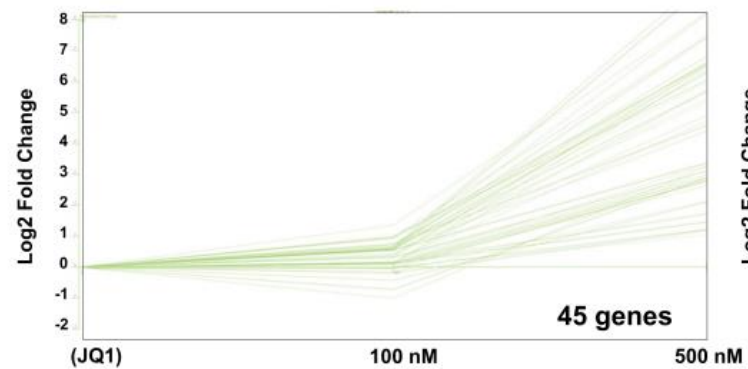

B

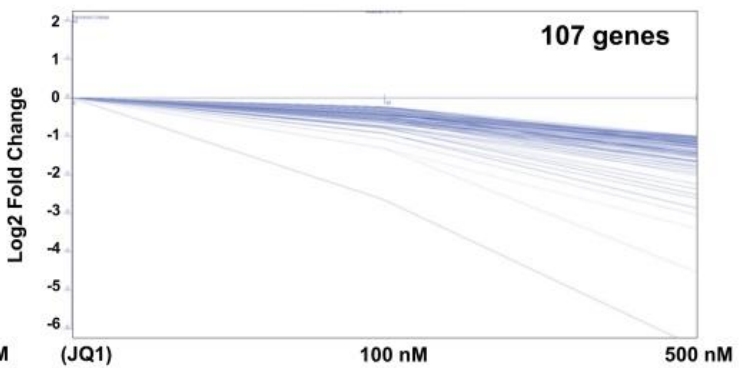

D

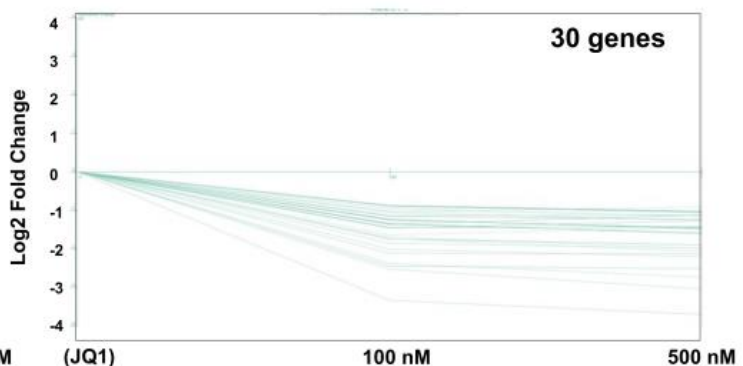

E

BRD4 occupancy and JQ1 responsiveness

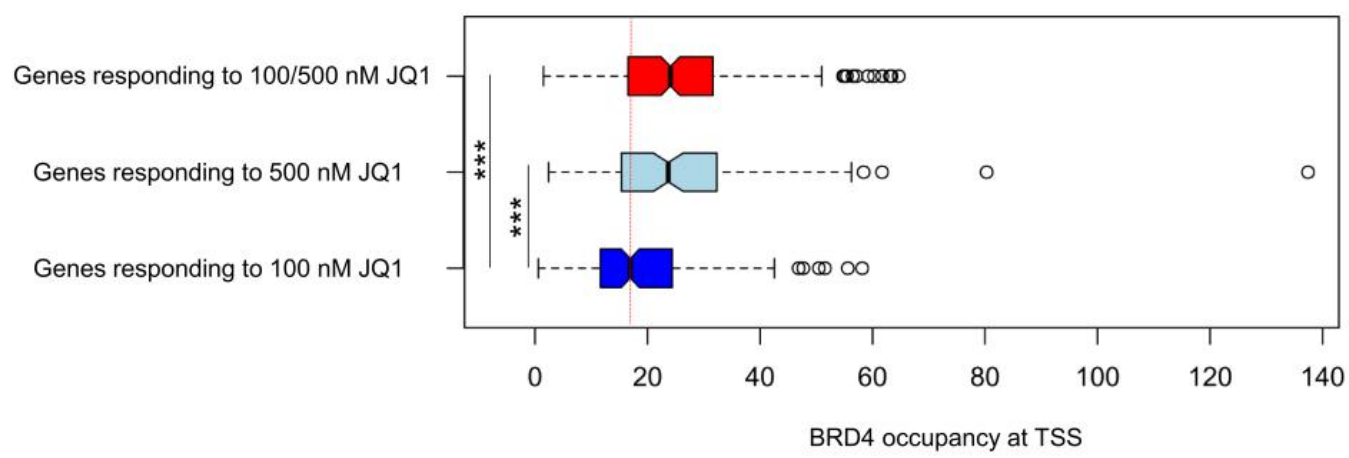

$\mathbf{F}$

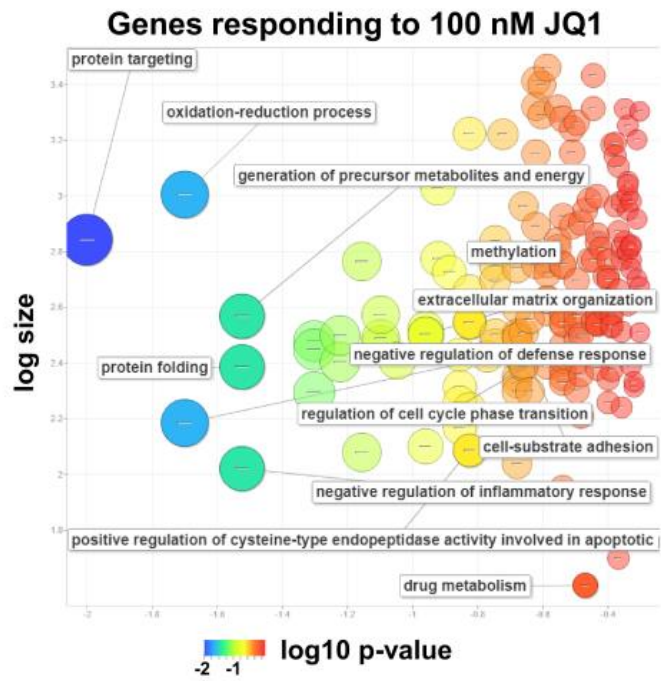

G

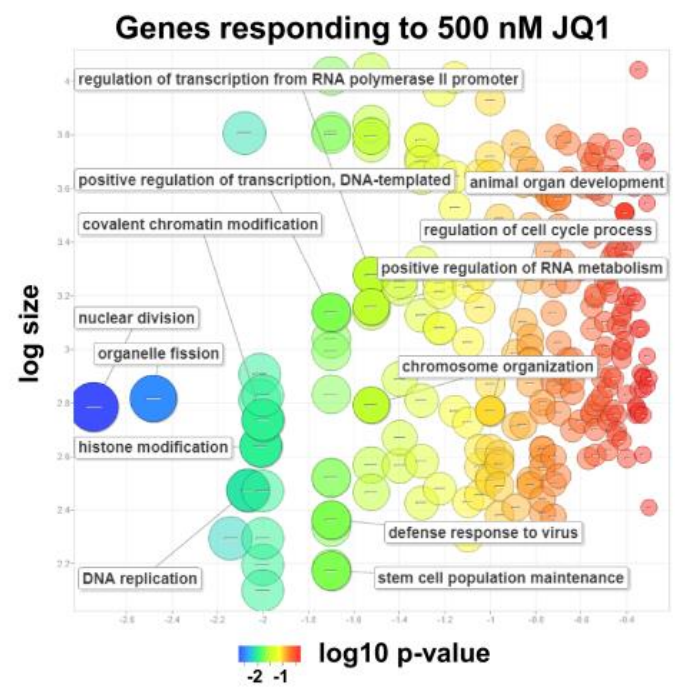


Figure 30 : BET-dependent genes respond differentially to low and high doses of JQ1. (A-D) Expression plots showing different clusters of genes sharing response patterns to low and high concentrations of JQ1. $x$-axis shows concentration of JQ1 and y-axis stand for fold change compared to cells treated with DMSO. (E) Box-plot showing the occupancy of BRD4 at the TSS of the genes of different clusters. ${ }^{* * *}$ stands for $p$-value $<0.0001$ for unpaired t-test. $(\mathbf{F}, \mathbf{G})$ Gene ontology plot for clusters of genes that are more responsive to low concentration (F) and high concentration (G).

\subsection{Discussion}

We report in this study the sensitization of pancreatic cancer cells to paclitaxel by low concentrations of the BET inhibitor JQ1. Combining JQ1 with chemotherapeutic agents has already been under investigation and showed promising results in various types of malignancies [443-447]. In particular, anti-microtubule drugs exhibited the most potent synergism with BET inhibitors in a screen of hundreds of agents in neuroblastoma [448]. While the anti-proliferative effect is usually used for JQ1 as a readout for efficiency, we showed that JQ1 sensitization can be exhibited in very low concentrations that have modest effects on growth. This indicates that sensitization can be rather due the transcriptional modulatory effects of BET inhibition.

Although the changes accompanying and causing paclitaxel resistance in vitro are stochastic in nature, we observed a transcriptional activation of a pro-inflammatory program that was reported in other systems upon paclitaxel treatment and resistance [420, 422, 423]. BET inhibition was reported to attenuate inflammation in acute pancreatitis [449], heart failure [450], and a subset of chronic obstructive pulmonary disease (COPD) patients [451]. It is not clear whether activation of inflammation is predisposing or accompanying to resistance. In any case, the fact that TNFa inhibition synergizes with BET inhibition in sensitizing pancreatic cancer to paclitaxel implies a causative role of inflammation in the development of resistance [426]. Additionally, the reversal of the enrichment for a migratory phenotype coupled with increased 
expression for the mutation inducers from the APOBEC family in resistant cells further justifies the investigation of BET inhibitors as an adjuvant therapy.

Interestingly, we observed a high association between BRD4 occupancy at putative enhancers and activation of associated genes in resistant cells. This was accompanied by the observation that some of the gained BRD4 regions at enhancers were associated with an open chromatin state in normal cells. Enhancers associated with open chromatin regions that are activated upon metastasis were reported in pancreatic cancer [154]. We also report here that enhancers associated with resistance may also be open before activation in case of resistance. This may imply that these regions are primed for activation and may explain why pancreatic cancer frequently displays innate or acquired resistance in a shorter period compared to other tumor types. It would be of interest to identify such regions and evaluate the potential of using their "openness" to predict the development of resistance and potentially the sensitization with BET inhibition. Moreover, enhancer RNA (eRNA) transcribed at these particular enhancers can also provide us with unconventional biomarkers for prognosis and therapy [103].

Given that the gain of BRD4 has also resulted in the identification of resistance-specific super enhancers driving genes with poorer prognosis, other therapies that specifically target this special class of enhancers can be highly beneficial in resistant pancreatic cancer. For example, inhibition of Cyclin Dependent Kinase 7 (CDK7), a subunit of TFIIH, has been shown to extensively affect the activity of super enhancers in neuroblastoma and ovarian cancer [452, 453]. Additionally, histone deactylases inhibitors (HDACs) were reported to affect super enhancer-driven transcription [331]. Accordingly, these and other inhibitors can potentially be used to manage patients with resistance to de-activate SE-driven transcriptional activation and improve prognosis. 
To evaluate the effect of low concentrations of JQ1 in sensitization, we compared the gene transcriptional profile of normal cells upon treatment with low and high concentrations of JQ1. Interestingly, various clusters of genes showed different patterns of responsiveness to the different concentrations. So far, dose escalation studies for BET inhibitors only takes into consideration the anti-proliferative and side effects of those agents and not their transcriptional modulatory effects. Thus, the rationale for variant sensitivity of genes to different concentrations of BET inhibitors is not yet known. As JQ1 targets both the BD1 and BD2 of the BET family members, we propose a bivalent pattern of inhibition which can be mirrored in dosedependence. For example, if only one of the bromodomains is bound at certain genes, they would respond to lower concentrations. On the other hand, if the two bromodomains are required to be occupied by acetylation at certain regions, higher concentrations of JQ1 may be needed for ejection of the BET family member from these regions (scheme in Figure 31A). This hypothesis can be tested by using a monovalent selective BET inhibitor for each bromodomain and investigating the profile of each. If this theory is true, the genes which respond to high concentrations would be resistant to either monovalent inhibitor but responsive to co-treatment. Another explanation for various dose responses can be due to the various occupancies of transcription factors at these regions. It can be possible that other transcription factors which are not BET-dependent can compensate for the absence of BET members at some regions and ensure the activation of the genes irrespective of the concentration of BET inhibitor (Figure 31B). Additionally, genes that are responsive to high concentrations may be highly occupied by BRD4, requiring a higher concentration for an effect to take place (Figure 31C). This is further supported by our observation that highly responsive genes have lower occupancy of BRD4 at their TSS. 
A
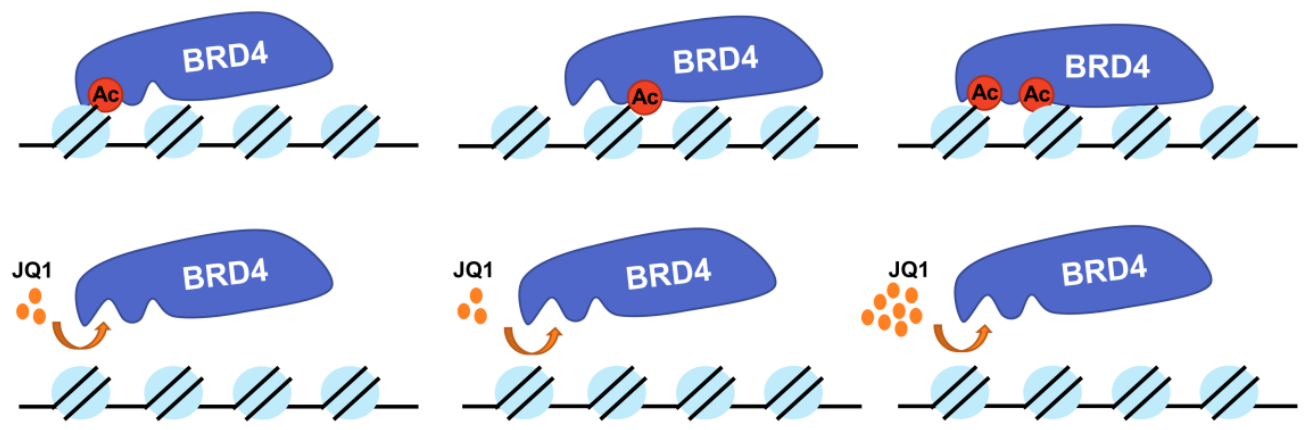

B
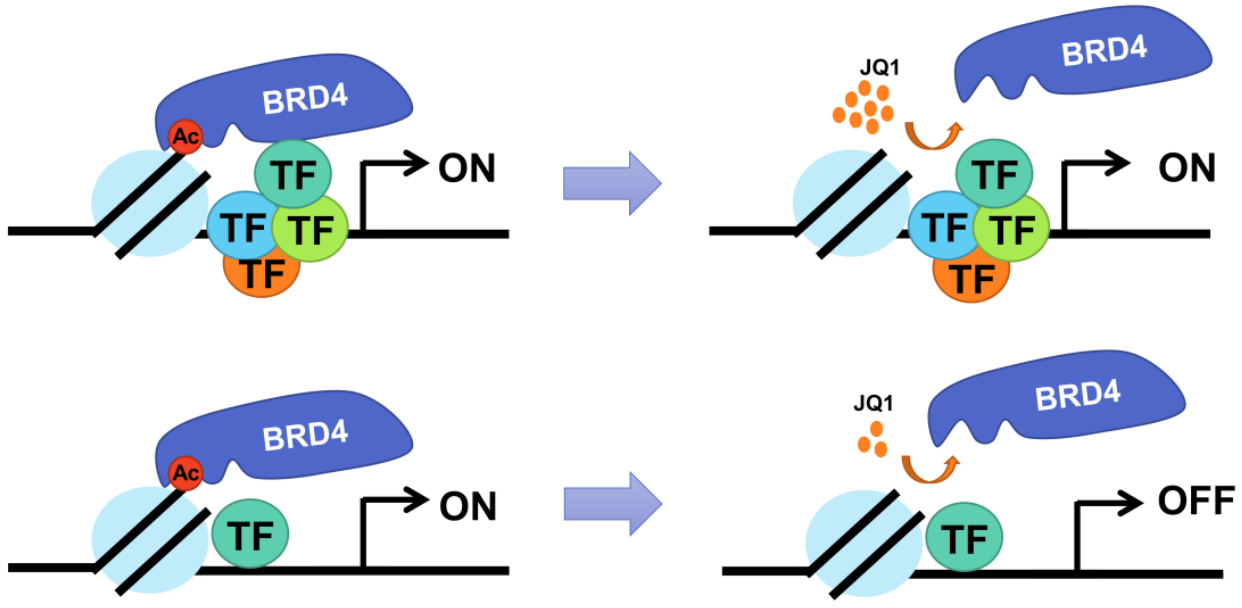

C
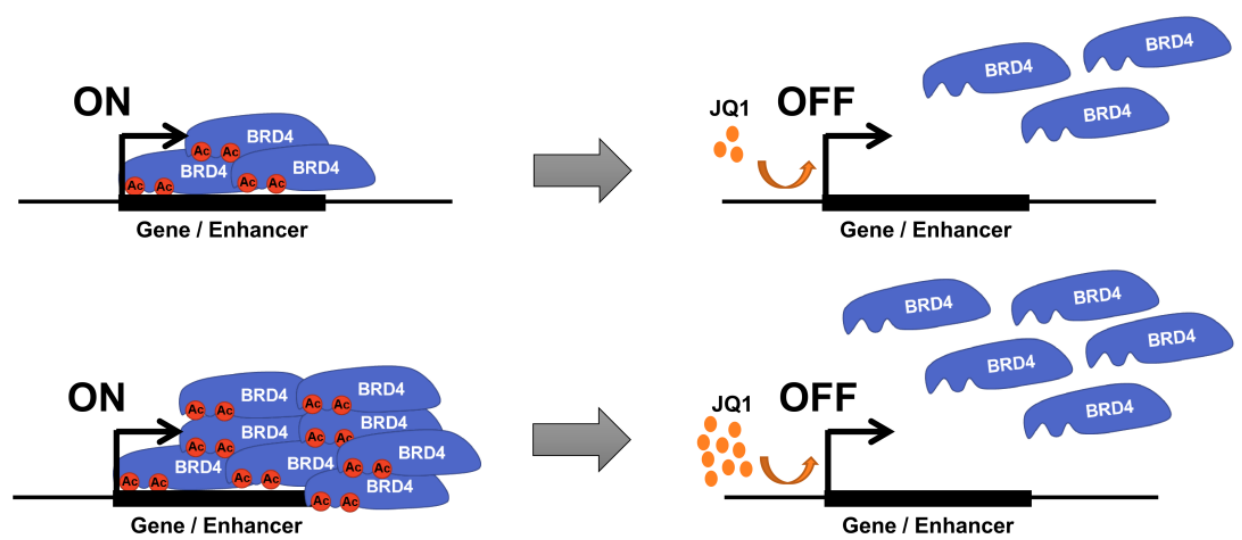

Figure 31 : Graphical representation for hypotheses explaining dose-dependent response for BET inhibitors. (A) Genes occupied in a bivalent manner require higher concentrations to be affected. (B) Other activating transcription factors can compensate for absence of BRD4. (C) Occupancy of BRD4 can affect response with lower occupancy leading to high response. 
In conclusion, as BET inhibitors are under investigation for their role as therapeutic management options for patients, insights into their mechanism of action and the optimal approaches to use them are currently lacking. We propose BET inhibitors as good options for sensitization to paclitaxel in both innate and acquired settings. These combinations however should be approached with the goal of leveraging the gene transcription modulating effects of these inhibitors rather than primarily their anti-proliferative effects in isolation.

\subsection{Materials and Methods}

\subsubsection{Cell Culture}

L3.6pl pancreatic cancer cells [378] were maintained in minimum essential media (MEM; Thermo Fischer scientific) supplemented with 10\% FBS (Thermo Fischer scientific), 1\% Penicillin/streptomycin (Sigma-Aldrich), and 1\% L-Glutamine (Sigma-Aldrich). For establishment of resistant cells, paclitaxel (T7191; Sigma-Aldrich) diluted in dimethyl sulfoxide (DMSO) (Roth) was added to the cells starting from $2 \mathrm{nM}$ (the estimated IC50). Once the cells started thriving, the concentration of paclitaxel was doubled till the cells were able to grow in $64 \mathrm{nM}$ after 4 months from starting with the lowest concentration. Subsequently, resistant cells were maintained in $64 \mathrm{nM}$ of paclitaxel in MEM media at all times.

\subsubsection{Proliferation assays}

For proliferation assays in 96 well plates, 1,000 cells were seeded in each well and treatment was performed every 48 hour in quadruplicates for each condition. Confluence was measured every day using Celigo Imaging Cell cytometer (Nexelecom Bioscience) and normalized on confluence of day 1. IC50 was extrapolated from dose-response curves using GraphPad Prism 4. Crystal violet experiment was performed in 24 -well plates where 5,000 cells were seeded and 
treatment was performed every 48 hours. After 8 days, cells were fixed with methanol, stained with $1 \%$ Crystal Violet in $20 \%$ ethanol, washed with water, and dried.

\subsubsection{RNA isolation and cDNA synthesis}

Cells were treated for 24 hours before RNA isolation. QIAzol Lysis Reagent (Qiagen) was added to the cells in addition to $100 \mu$ l of chloroform and centrifuged at full speed for $30 \mathrm{~min}$. The aqueous phase was kept and mixed with an equal volume of chloroform and centrifuged for 20 minutes. The aqueous phase was then kept and the RNA was allowed to precipitate in isopropanol at $-20^{\circ} \mathrm{C}$ overnight. Subsequently, RNA was washed twice with $70 \%$ ethanol and resuspended in RNase free water. Complementary DNA (cDNA) was synthesized from $1000 \mathrm{ng}$ of RNA by mixing with $1.875 \mu \mathrm{M}$ of random 9mer primers (Sigma-Aldrich) and $625 \mu \mathrm{M}$ deoxyribonucleotides dNTPs (Jena Bioscience) in a total volume of $16 \mu \mathrm{l}$ and heated at $70^{\circ} \mathrm{C}$. Then, the mix was added to 10 units of RNase inhibitor (New England Biolabs) and 25 units of M-MuLV Reverse transcriptase (New England Biolabs) in 1X reaction buffer and incubated at $42^{\circ} \mathrm{C}$ for 1 hour. The enzyme was then inactivated by incubation at $95^{\circ} \mathrm{C}$ for 5 minutes. The resulting DNA was then diluted to a total volume of $50 \mu \mathrm{l}$ of water.

\subsubsection{Library preparation for RNA-seq}

The integrity of RNA was validated by gel electrophoresis and 500ng were used to make the libraries in triplicates for each condition. Libraries were made using the TruSeq RNA Library Prep Kit V2 (Illumina) according to the manufacturer's instructions. Briefly, Oligo-dT beads were used to capture poly-A tailed-mRNA followed by first strand cDNA synthesis by Superscript II reverse transcriptase (Thermo Fischer scientific). Second strand synthesis was followed by end repair, 3' adenylation, adapter ligation and library amplification. Agencourt AMPure XP (Beckman Coulter) was used for size selection during the library synthesis. The quality of the 
resulting DNA was measured with high sensitivity DNA kit (Agilent) on the Agilent Bioanalyzer 2100. Samples were sequenced (single-end 50 bp) on a HiSeq4000 (Illumina) in the Transcriptome and Genome Analysis Laboratory (TAL) at the University Medical Center Göttingen. Images of sequences were converted into bcl files (BaseCaller software, Illumina) and demultiplexed to fastq files by CASAVA v1.8.2.

\subsubsection{Quantitative real time PCR (qPCR)}

$1 \mu \mathrm{l}$ of the cDNA reaction was mixed with $600 \mathrm{nM}$ primer mix, $8.5 \mu \mathrm{l}$ of water and $14 \mu \mathrm{l}$ of a home-made PCR mix (3 mM MgCl $2,800.000 X$ Sybr Green (Eugene), $200 \mu \mathrm{M}$ dNTPs, 0.5 unit Taq polymerase (Primetech), 0.25\% Triton-X-100, $300 \mu \mathrm{M}$ Trehalose, $75 \mathrm{mM}$ Tris-HCl (pH 8.8), $20 \mathrm{mM}(\mathrm{NH} 4)_{2} \mathrm{SO} 4,0.01 \%$ Tween 20) [391]. The PCR program run comprised of 2 minutes of initial denaturation at $95^{\circ} \mathrm{C}$ followed by 40 cycles of 10 seconds at $95^{\circ} \mathrm{C}$ and 30 seconds at 60 ${ }^{\circ} \mathrm{C}$. Gene expression changes were evaluated using the starting quantity method and plotted on GraphPad Prism 4. Each condition was evaluated in biological triplicates and technical duplicates. Primers used in this study included:

\begin{tabular}{|c|c|c|}
\hline Gene & Forward sequence $\left(5^{\prime}-3^{\prime}\right)$ & Reverse sequence (5'-3') \\
\hline APOBEC3C & TGAATCCACAGATCAGAAACCCG & ACTGAGCGGCGCTTTATACC \\
\hline CXCL5 & AGAGAGCTGCGTTGCGTTT & TTCTTCAGGGAGGCTACCACTT \\
\hline FST & ATCCCCTGTAAAGAAACGTGTGA & GCGGTAGGTTTTCCCATCCA \\
\hline LIF & TCCCGGCTAAATATAGCTGTTTCTG & CCGGCAGTTTTCAGAGGTTCA \\
\hline SEMA3C & TCGGAACAGATCGGGGTACT & AGAATGAGCTCGCCACTGAC \\
\hline TNFAIP2 & TACCCTGACTTCAGCAAAGGC & CTGACGTCCAAGATGCTCCG \\
\hline
\end{tabular}

\subsubsection{Chromatin immunoprecipitation (ChIP-seq)}

Chromatin immunoprecipitation was performed as described previously [99, 152]. Briefly, crosslinking was performed using 1\% formaldehyde for 20 minutes and nuclear pellets were sonicated in sonication buffer $(150 \mathrm{mM} \mathrm{NaCl}, 20 \mathrm{mM}$ EDTA, $50 \mathrm{mM}$ Tris-HCl (pH 8), 1\% v/v NP40, 0.5\% v/v sodium deoxycholate, $20 \mathrm{mM} \mathrm{NaF}, 0.1 \%$ SDS) for 30 cycles using a Bioruptor Pico 
(Diagenode) and a cycle setting of $30 \mathrm{~s}$ on/off. Samples were precleared by $50 \%$ slurry of

Sepharose 4B (GE Healthcare) and incubated with antibody overnight. Antibodies included

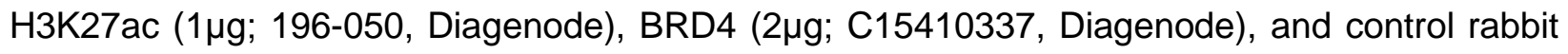

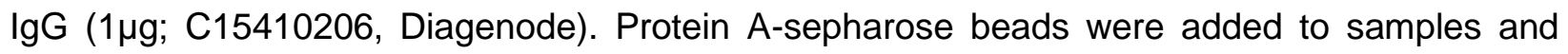
incubated for $2 \mathrm{~h}$, then washed, de-crosslinked, and DNA was extracted. Samples were performed in duplicate for each condition. Libraries were prepared from $0.5 \mathrm{ng}$ of immunoprecipitated DNA using the MicroPlex Library Preparation Kit v2 (Diagenode) according to manufacturer's protocol. DNA integrity was measured with high sensitivity DNA kit (Agilent) on the Agilent Bioanalyzer 2100. Samples were sequenced (single-end 50 bp) on a HiSeq4000 (Illumina) in the Transcriptome and Genome Analysis Laboratory (TAL) at the University Medical Center Göttingen.

\subsubsection{Chem- seq for biotinylated JQ1}

Chem-seq was performed according to Anders et al with minor modifications [454]. Briefly, cells were crosslinked for 20 minutes with $1 \%$ formaldehyde and quenched with Tris- $\mathrm{HCl}(\mathrm{pH}$ 7.5) for a final concentration of $300 \mathrm{mM}$. Cells were washed twice with cold PBS and re-suspended in Chem Lysis Buffer (50 mM HEPES (pH 7.5), $140 \mathrm{mM} \mathrm{NaCl,} \mathrm{1mM} \mathrm{EDTA,} \mathrm{10 \%} \mathrm{Glycerol,} \mathrm{0.5 \%}$ NP-40, 0.25\% Triton X-100). Samples were consequently washed with Chem washing Buffer (10 mM Tris- $\mathrm{HCl}$ (pH8), 1mM ethylenediamine tetraacetic acid (EDTA), $0.5 \mathrm{mM}$ ethylene glycol tetraacetic acid (EGTA) $200 \mathrm{mM} \mathrm{NaCl}$ ). Samples were then sonicated in Chem sonication Buffer (50 mM HEPES-KOH (pH 7.5), 140 mM NaCl, 1 mM EDTA, 1 mM EGTA, 1\% Triton X-100, $0.1 \%$ Sodium Dodecyl Sulfate (SDS), $0.1 \%$ sodium deoxycholate) for 30 cycles (30s on/off).

All buffers included $100 \mu \mathrm{M} \beta$-glycerophosphate disodium salt hydrate (BGP), $100 \mu \mathrm{M} \mathrm{N}$ ethylmaleimide, $1 \mathrm{nM}$ iodoacetic acid, and protease inhibitors (100 $\mu \mathrm{M}$ Pefabloc, $1 \mu \mathrm{M}$ aprotonin, 
$1 \mu \mathrm{M}$ leupeptin). Magnetic streptavidin Dynabeads (Invitrogen) were incubated with $200 \mu \mathrm{M}$ biotinylated JQ1 (Kindly provided by James Bradner) or DMSO in $0.5 \%$ bovine serum albumin (BSA) in PBS at a rotating wheel for 6 hours. Consequently, sonicated samples were incubated with the magnetic beads for 20 hours. Next, the beads were washed twice with sonication buffer, once with high-salt sonication buffer (with $500 \mathrm{mM} \mathrm{NaCl}$ instead of $140 \mathrm{mM}$ ). This was followed by one wash with Chem LiCl Buffer (20 mM Tris- $\mathrm{HCl}$ (pH 8), 1 mM EDTA, 0.5\% NP-40, $0.5 \%$ sodium deoxycholate, $0.1 \%$ SDS, and $250 \mathrm{mM} \mathrm{LiCl}$ ) and one final wash with Chem TE Buffer (10 mM Tris-HCl (pH 7.5), $0.1 \mathrm{mM}$ EDTA). Consequently, DNA was isolated with phenol/chloroform. $1 \mathrm{ng}$ of the extracted DNA was used for library preparation using Microplex Library kit (Diagenode) according to manufacturer's protocol. Quality of resulting DNA was checked on the Agilent bioanalyzer and samples were sequenced as mentioned previously for ChIP-seq samples.

\subsubsection{Bioinformatic analysis of RNA-seq}

Bam files were generated by mapping raw reads by TOPHAT/2.1.0 and the annotation file for hg19 which was exported from the UCSC table browser [404, 405]. Differential gene expression analysis was performed by CUFFLINKS/2.2.1 using cuffdiff to evaluate log fold changes between condition and cufflinks to calculate Fragments per kilobase per million (FPKM) values [406]. Euclidean distance was used to hierarchically cluster genes using cluster 3.0 [407] and the resulting Heatmap was viewed by Gene TreeView 3.0 [408]. Gene set enrichment analysis (GSEA) was performed with default settings using FPKM values of expressed genes as an input [409]. Gene expression patterns in response to different conditions were clustered by Short Time-series Expression Miner (STEM) [455] with the built-in gene ontology for the clusters used to generate the gene ontology plots viewed by REVIGO [456]. 


\subsubsection{Bioinformatic analysis of ChIP-seq}

Mapping of reads was performed to the reference genome assembly (hg19) by BOWTIE2/2.2.5 [359]. The resulting sam files were converted to bam files then sorted and indexed using SAMTOOLS/1.4 [360]. The bamCoverage tool in DEEPTOOLS/2.4.0 was used to normalize reads to $1 \mathrm{X}$ sequencing depth [361]. Bigwig files were generated by ignoring duplicates and extending to $200 \mathrm{bp}$ and were viewed using the Integrative Genomics Viewer (IGV 2.4) [362, 363]. MACS2/2.1.1.20160309 was used to call the significant peaks without building the shifting model and with --broad-cutoff 0.05 for BRD4 and H3K27ac and input files from respective cells as background [393]. The Bioconductor $\mathrm{R}$ package Diffbind was run on $\mathrm{R}$ version 3.3.1 according to the instruction manual to define regions that gained BRD4 upon paclitaxel resistance [396]. Associated genes with specific regions were identified using Genomic Regions Enrichment of Annotations Tool (GREAT) analysis with default settings. Occupancy of BRD4 was evaluated by the computeMatrix tool on the Galaxy platform and the average profiles and heatmaps were generated based on computeMatrix values with the plotheatmap tool with the reference point selected as the peak center [397]. Boxplot were plotted using the boxplot function in R 3.3.1. The ROSE algorithm was used to identify super enhancers from stitched regions of $\mathrm{H} 3 \mathrm{~K} 27 \mathrm{ac}$ regions and BRD4 signal with the default settings with ignoring regions that are 2500 bp around the TSS [123, 124]. Upstream activators for regions that gained BRD4 in resistant cells were identified using EnrichR web-based interface [401]. Motif analysis was performed using the findMotifs.pl command in HOMER/4.8 with the background as shuffled sequences prepared from the same files with scrambleFasta.pl [457]. 


\subsubsection{Statistical analysis}

The Mantel-Cox test was used to indicate significance for Kaplan-Meier plots. Significance in

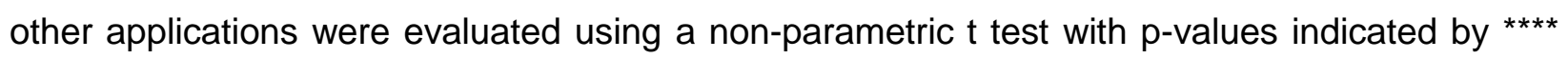
for $=<0.0001,{ }^{* * *}$ for $=<0.001,{ }^{* *}$ for $=<0.01$ and ${ }^{*}$ for $=<0.05$.

\subsection{Data availability}

Sequencing data available upon request.

\subsection{Acknowledgments}

The authors would like to thank G. Salinas, F. Ludewig, and S. Lutz from the Transcriptome and Genome Analysis Laboratory of the University Medical Center Göttingen, Germany for performing the next generation sequencing for ChIP-seq, RNA-seq, and Chem-seq data; James E. Bradner for kindly providing biotinylated JQ1; Dobbelstein Group for providing access for their cytometer; G.E. Schmidt; F. Wegwitz; X. Wang; Jana Henck and all the members of the Johnsen group for their support and discussions.

\subsection{Authors contribution}

F.H.H. performed proliferation assays, established resistant cells, next-generation sequencing including ChIP, RNA, and Chem-seq and all bioinformatic analysis. A.P.K performed quantitative real-time PCR and crystal violet assays. S.A.J. and F.H.H. designed experiments and conceived the ideas for this project. F.H.H. and S.A.J wrote this manuscript.

\subsection{Funding}

This work was supported by the German Academic Exchange Service (DAAD) to F.H.H.; Deutsche Krebshilfe (PiPAC Consortium) [70112505] and the Deutsche Forschungsgemeinschaft (DFG) [JO 815/3-2] to S.A.J. 


\subsection{Conflict of interests}

The authors declare no conflict of interest. 


\section{CHAPTER 6}

\section{General Discussion}

This chapter provides a general discussion of the general themes in this project including the future prospects.

No published data is included in this chapter. 


\section{General Discussion}

\subsection{Enhancers as an emerging therapeutic target in malignancy}

In this project, we investigated the role of enhancers and super enhancers in different contexts of malignancy. Expansion of our understanding of complex processes controlling gene expression regulation will undoubtedly uncover numerous novel targets that can be perturbed and modulated in cancer. Focusing on targets involved in such processes is highly rational due to cancer addiction to aberrant transcriptional regulation [271]. On the other hand, such exponential growth in knowledge will render the task to invest in a select few of effective and relatively safe transcriptional targets immensely challenging.

A hypothetical "ideal" transcriptional target in cancer therapy would necessarily exhibit certain attributes which can lead to a perceptible change in the quality of life, prognosis and the therapeutic management of patients. To do so, a target should be firstly easily modulated, preferably by small molecule inhibitors that can be made easily available at target site with an acceptable half-life. Most importantly, such target should be highly specific sparing normal cells from further gene transcriptional modulation, thereby insuring the lack of induction of unwanted anomalies in healthy tissue and decreasing the severity and gravity of resulting adverse effects. Additionally, this ideal target should be indispensable to cancer cells rendering them highly dependent on such target. Ideally, this dependence should be shared by all or a high percentage of the malignant cell population. This dependence should not necessarily cause cell death but could alternatively lead to a higher sensitivity to other less specific therapies such as radio/chemotherapy. Moreover, a compensatory mechanism circumventing the need for such target should be unattainable or at least targetable. Furthermore, a biomarker to predict the rate of success of this target prior to and succeeding its use should be available and easily tested. 
While identification of an ideal transcriptional target may not realistically be possible, current available targets can be considered highly promising if they exhibit more of its aforementioned traits. In concordance with this, enhancers emerge as a highly promising target in malignant diseases. Enhancers were shown to be easily targeted by numerous small molecular inhibitors. Interestingly, we have uncovered in Chapter 5 a sensitivity of enhancers gained in paclitaxel resistant cells to the BET-inhibitor, JQ1. Preferential dependence of enhancers on BET proteins has been consistently reported in various diseases such as pancreatic cancer [212], T-cells in auto-immune diseases [458], leukemia [459], multiple myeloma and glioblastoma [123]. Other modulators with reported efficacy on enhancers include CDK7 inhibitors [453], HDAC inhibitors [460], and MEK inhibitors [461]. Accordingly, our study in chapter 5 adds to the existent evidence of the targetability of enhancers with molecular inhibitors.

One of the major characteristics of a subgroup of enhancers is its context-specificity. As we have shown in chapter 3 , enhancers of the $M Y C$ gene showed system-specificity where the enhancer CCAT1 is responsible for activating MYC in colorectal cancer. Alternatively, PCAT1 enhancer is active in prostate cancer in contrast to PVT1 enhancer in leukemia. Such a systemspecific pattern of enhancer activation indicates a selectivity that can be positively harnessed in targeted therapy. Not only do enhancers show specificity between systems, but this extends to molecular subtypes in the same malignancy. In chapter 4, we showed that enhancers are enriched in a particular molecular subtype of pancreatic cancer and defines its molecular identity. This further proves the highly selective and identity determining role of enhancer elements in disease. Furthermore, we reported in chapter 5 the enrichment of enhancers upon development of resistance to paclitaxel which further implicates enhancer activation in diverse phenotypes, in this example resistance, in the same cell line. All our observations in the 3 chapters are consistent with the established pattern of context-specificity of enhancers [462]. 
Notably, many enhancers were identified as indispensable for driving oncogenic programs in diverse cancer types. For example, a chromosomal rearrangement in GATA2 distal enhancer led to the activation of stemness inducing ecotropic virus integration site-1 (EVI1) and resulted in the development of leukemia [136]. Additionally, the known oncogene MYC was also shown to be driven by a BET-dependent enhancer in colorectal and esophageal carcinomas [251, 463]. Furthermore, hijacked enhancers led to the activation of the oncogenic growth factor independent 1 family in medulloblastoma [464]. In this project, we have shown in chapter 4 that deltaNp63 leads to the activation of enhancers driving a squamous phenotype and associated with a worse prognosis. Additionally, we uncovered in chapter 5 the activation of proinflammatory and migratory programs by enhancers. Altogether, our findings support that enhancers are a bone fide activators of aberrant transcriptional programs underlying malignancy.

While targeting transcriptional enhancers is still under investigation, compensatory mechanisms upon inhibition of active enhancers have already been described. For example, the BET inhibitor, JQ1, was reported to induce resistance mediated by transcriptional activation in bromodomain independent pathways in castration-resistant prostate cancer [432]. Interestingly, this resistance uncovered an alternative dependency on CDK9-mediated activation of androgen receptor signaling. Additionally, acquired resistance to MEK inhibition was compensated for by BET-mediated activation that can be easily targeted by BET inhibition in triple-negative breast cancer [465]. It is probable that even after the use of BET inhibitors along with chemotherapy as we recommend in chapter 5 , cancer cells will find a way to evade the specific transcriptional block brought by BET inhibition by activating other pathways. Accordingly, it is of highest importance to try and identify these activated pathways upon acquired resistance and investigate new approaches to target them. Extended experience with using inhibitors to target 
enhancers coupled with increased knowledge about different pathways to activate transcription can help guide a more precision-based and most likely a multi-layered approach in patient therapeutic management.

Finally, as discussed in Chapter 2, while a particular function for eRNA has not been conclusively identified, we were able to reasonably identify super enhancers by their eRNA signal. As we already indicated, this implies that eRNA can play a potential role as biomarkers for enhancer activity. This is of particular interest as eRNA were reported to be highly enriched at tissue-specific enhancers [466]. Indeed, the eRNA CCAT1 was proposed as a therapeutic biomarker that can predict responsiveness to BET inhibition [251]. Interestingly, the largely known marker of prostatic cancer, Prostate-specific antigen (PSA), was identified to be associated with an enhancer with the resulting eRNA having a prominent role in gene transcriptional regulation [467]. Additionally, Kaczkowski et al. [468] identified 90 eRNAs that are generally upregulated in cancer cells upon screening over 200 cell lines and approximately 300 primary cell samples. Thus, based on our results in chapter 5, eRNAs can have a potential role as biomarkers for resistance and responsiveness to BET inhibition sensitization.

In conclusion, enhancers exhibit many attributes of the "ideal" transcriptional target and are highly promising to be leveraged in cancer therapy and management. This is due to the fact that they are targetable, specific, indispensable, provide biomarkers (eRNA), and the compensatory mechanism in response to their modulation can be targeted as well (Figure 32). However, the application of inhibitors targeting enhancers will face few hurdles upon application in the clinics. Enhancers are usually identified using highly complex bioinformatic analysis that is not always accessible to clinicians or scientists alike. Additionally, targeting enhancer elements can be either detrimental or beneficial depending on the context. As cancer is a heterogeneous disease, this might inadvertently lead to unwanted effects in a select population of cells. 
Furthermore, the long term repercussions of transcriptional modulation, even in normal cells which theoretically lack the dependence on these activated enhancers, are yet to be defined. These challenges should be considered while investigating the targeting of enhancers in cancer amongst other diseases.
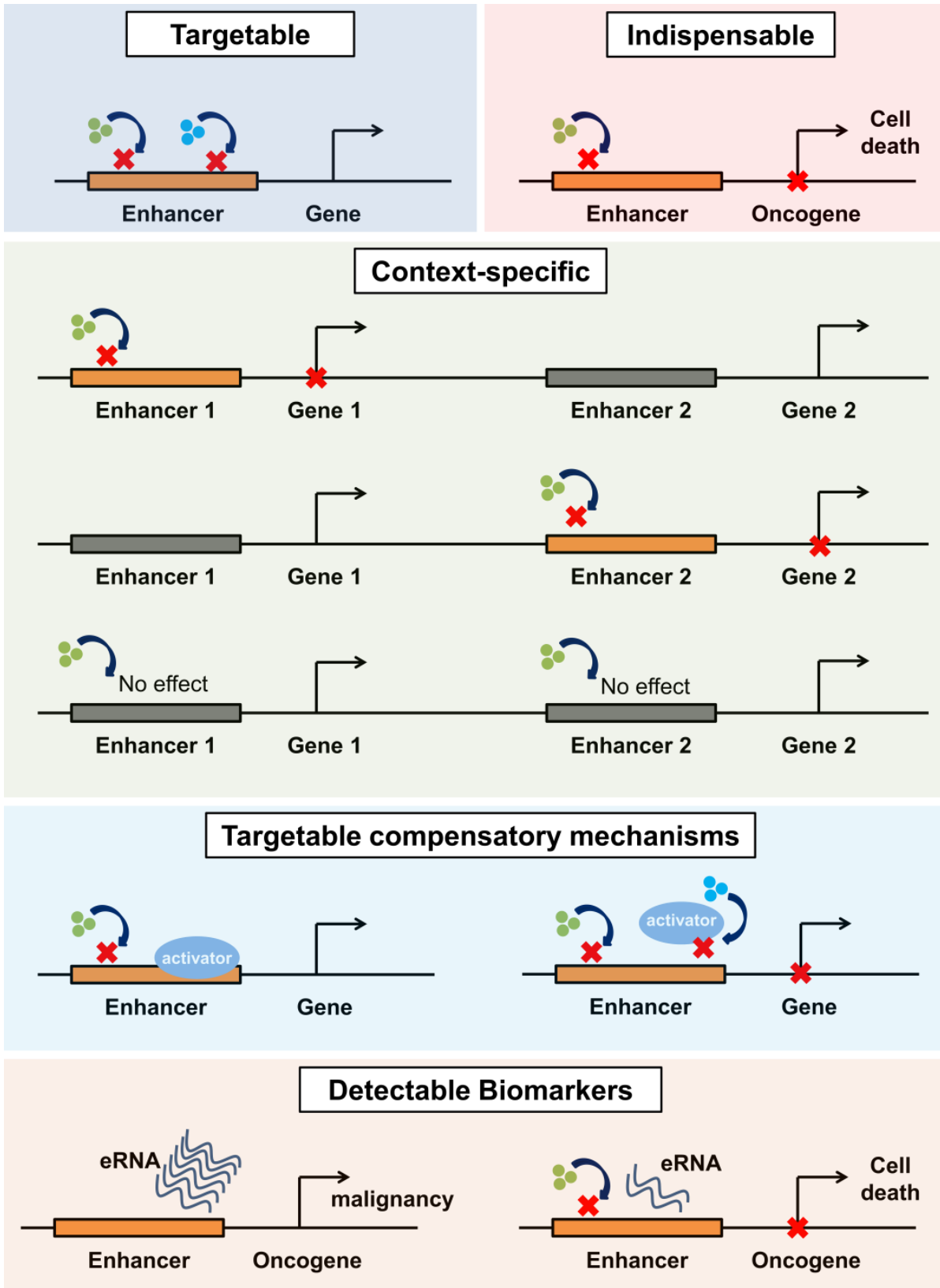

Figure 32 : Positive attributes of enhancers leading them to be good candidates for targeting in cancer. Grey enhancers are inactive and orange active. 


\subsection{Super enhancers as an important subset of enhancer elements}

Since the recent identification of super enhancers in 2013 [123, 124], approximately 300 scientific papers discussing this subclass have been published. In this project, we endeavored to study the role of super enhancers as part of our general study of distal regulatory elements in cancer progression and disease. In chapter 2, we used publicly available data from the ERpositive cell line MCF7 to investigate if super enhancers have indeed higher efficacy compared to typical enhancers. This provided us with a system that is highly dependent on enhancers to mediate its effects. Our analysis failed to uncover a particular advantage of super enhancers over typical enhancers in the percentage of genes affected but identified major ER targets to be driven by adjacent super enhancers. This has also been observed in embryonic stem cells where deletion of various super enhancers had variable extent of effects on transcription of target genes [469]. However, a big limitation in our study is that we used simple gene associations based on proximity. A better approach would be to use newly available techniques that identify contact points between regions throughout the genome to ensure the accurate identification of target genes for super and typical enhancers [118]. As many neighboring genes may not be affected by an associated super enhancer, they may decrease the significant correlation seen when studying super enhancers and their target genes.

On the other hand, our studies of super enhancers in pancreatic cancer in the context of different molecular subtypes (chapter 4) and chemo-resistance (chapter 5) have identified a particularly important role for this subclass in driving gene regulation while applying the same association rule. It can be probable that super enhancers have diverse impacts in various contexts and that few systems are more dependent on super enhancers for activating particularly important programs. Future studies in different systems will uncover if super enhancers have such a preference in impact in certain contexts and settings. 
Super enhancers represent a lucrative target as they have shown particular sensitivity when modulated by certain inhibitors leading to perceptible effects. For example, super enhancers in esophageal cancer showed high dependence on the CDK7 inhibitor, THZ1 [470]. Interestingly, a subset of super enhancers in esophageal cancer was observed to be highly enriched with TP63 [463]. As we have identified deltaNp63-dependent super enhancers driving the more aggressive squamous phenotype in pancreatic cancer, it would be of high interest to check the sensitivity of squamous cell lines to THZ1 in comparison to cell lines of other molecular subtypes. This can also be investigated in PDX from patients stratified into different subtypes and have a promising potential as a therapeutic agent in the squamous molecular subtype. Notably, combining of THZ1 and JQ1 has shown increased effects on super enhancer deactivation in osteosarcoma [471]. The effects of combining THZ1 and JQ1 in paclitaxel-resistant cells is also of particular interest given the implication of super enhancers in activating pro-inflammatory and promigratory programs in resistant cells and the partial reprogramming of super enhancers in resistant cells. Additionally, analysis in acute myeloid leukemia uncovered a specific super enhancer in a subtype of patients that can be sensitive to an agonist leading to myeloid differentiation [472]. Accordingly, analysis should not only concentrate on super enhancers that can be silenced, but also on novel super enhancers that can be further activated leading to a better prognosis.

One of the challenges facing the study of super enhancers is the identification of less known enhancer subclasses which are not well defined. For example, stretch enhancers are sometimes used interchangeably with super enhancers [473, 474] while other studies indicate that stretch enhancers meet only the requirement of spanning long stretches of DNA and are not necessarily rich with transcription factors or cell-specific [475]. Shadow enhancers are a group of "secondary" enhancers that are redundant to an active enhancer and ensure the 
precision of gene transcriptional regulation [476]. Such a concept which was first identified in Drosphila has also been reported in mammals [477]. This led to the sometimes imprecise use of shadow enhancers to describe typical enhancers which are not necessarily supportive of other enhancers. A clear definition of these new classifications will significantly help in controlling the confusion that is usually associated with super enhancers.

Testing and understanding the settings of the algorithms used in identifying super enhancers was a major focus of this project. In chapter 2 , we have critically studied the various settings of the ROSE algorithm and questioned its biased approach for stitching enhancers at an already pre-defined distance. Interestingly, a new algorithm with a machine learning approach to define stitching distance was recently developed [130]. We have used both algorithms in identifying super enhancers and did not observe a big difference between them. To further optimize the process of identification of super enhancers, other types of data can be used. For example, occupancy data from more than one factor can be simultaneously incorporated to calculate the intensity at enhancers. By definition, super enhancers are highly enriched with transcription factors [248]. Thus, having high enrichment with more than one transcription or activating factor can uncover a highly effective subtype of super enhancers. Another optimizing possibility can be to include 3D genome data to account for the looping effect needed for enhancers to target their genes. While it is still possible that an enhancer can affect a target gene without direct interaction, e.g. eRNAs, it is still not studied if super enhancers particularly form a hub of interaction with many genes or other enhancers. The question arises if super enhancers will indeed have a higher interaction with many regions throughout the genome or if they exert their effects by simply controlling master upstream regulatory genes. Moreover, RNA-seq data from systems treated with inhibitors that preferentially target super enhances can be incorporated in the algorithms identifying super enhancers and may uncover new dependencies. Our main 
findings throughout this project includes identifying of important ER effectors as downstream targets of super enhancers, uncovering of DeltaNp63-dependent super enhancers as drivers for a squamous molecular subtype in pancreatic cancer, in addition to detecting super enhancers driving genes leading to poorer prognosis in paclitaxel-resistant cell. All these observations underscore the importance of super enhancers in driving crucial programs in the cell and support the increased interest in this particularly important subset of enhancers.

\subsection{Transcription factors circuitry in context-specific gene regulation}

One of the major findings in our project was the uncovering of a transcription factor circuit including $\triangle$ Np63, HIF1A, BHLHE40, and RXRA that underlies the activation of the squamous molecular subtype by deltaNp63-dependent super enhancers in pancreatic cancer. The pattern of regulating major programs related to molecular identities of cells via cooperating transcription factors was already observed in other systems. Driving somatic cells toward a more pluripotent identity was reported to be mediated by a group of cooperating transcription factors namely, POU Class 5 Homeobox 1, SRY-Box 2, and Nanog Homeobox (POU5F1/SOX2/NANOG) [478480]. Muscle specific transcription factors such as myogenic differentiation 1 (MyoD) and myocyte enhancer factor $2 \mathrm{~A}$ (MEF2A) cooperates in defining a muscular phenotype in the cell [481, 482]. Upon analysis of footprinting data from accessible chromatin assays in 41 different cells, networks comprising of interconnected transcription factors that are highly specific to each system could be easily predicted and identified [483]. Indeed, a pattern where a group of transcription factors are co-expressed leading to the differentiation of a cell into a certain identity provide a tightly-controlled gene regulation system.

Notably, transcription factor circuits are highly important due to the possibility of their modulation, thereby manipulating cell identity and crucial transcriptional programs. While 
targeting the direct interaction between transcription factors and DNA is quite challenging [484], transcription factor activity can be attenuated by targeting their interactions with other co-factors, their downstream pathways, or by decreasing their stability through increased rate of proteasomal degradation [485]. Perturbation of these transcriptional circuits may follow different patterns where targeting one transcription factor can disturb the whole circuit and largely affect downstream targets or only partially with the other factors compensating for the absence of one factor [486]. Thus, it would be of high interest to check which rule would the transcription factor circuit that we identified follow. This can be uncovered by knocking down the individual components of this circuitry and evaluating the effects on chromatin status (H3K27ac as marker of activation) along with the effects on target genes. It could be possible that those transcription factors have an additive effect or an all-or-none effect on activation of their target genes. A more collaborative effect meaning that the modulation of any of these transcription factors can lead to the shutdown of the squamous program would be highly promising for patients of this particularly aggressive subtype. Interestingly, few of the transcription factors which we identified can be chemically modulated. HIF1A can be targeted at many levels spanning its transcription, translation, protein stability and degradation [487]. While RXRA can also be targeted, it should be taken into consideration that it was the only gene that is associated with our defined subtypespecific super enhancers that correlated with better prognosis. Accordingly, targeting RXRA should be approached with caution as it might not lead to favorable effects in pancreatic cancer patients. Altogether, our work identifies promising new targets that underlie the development of more aggressive phenotypes. 


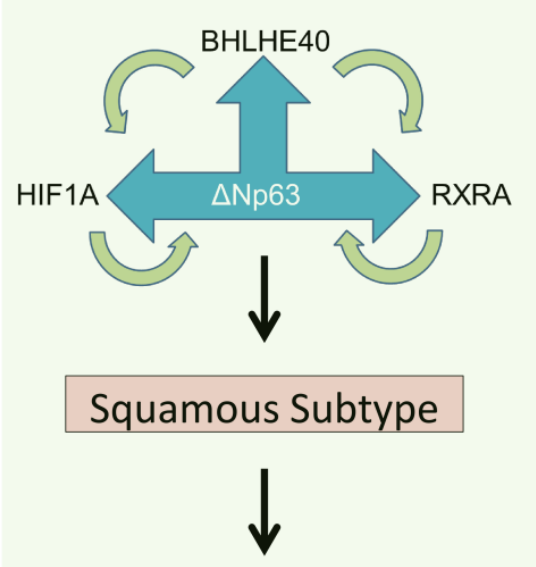

Worse prognosis

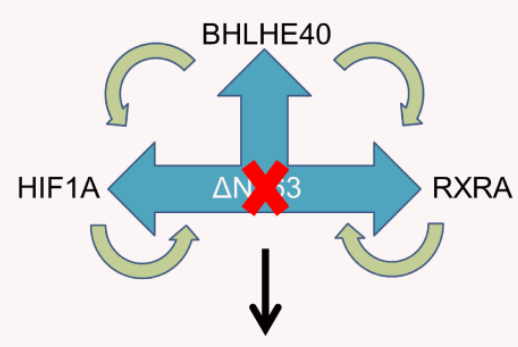

Squamo! Subtype

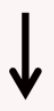

Better prognosis

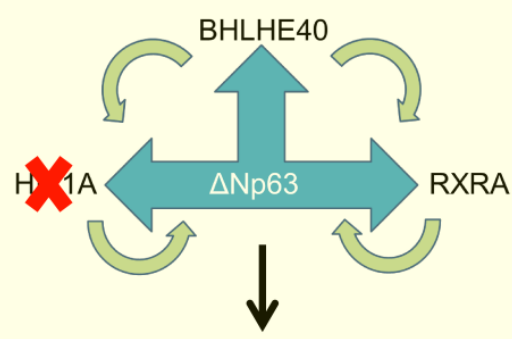

?? Squamous Subtype

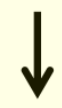

??

Figure 33 : The transcriptional factor circuitry driven by deltaNp63-dependent super enhancers in the squamous molecular subtype of pancreatic cancer. Upon knockdown of deltaNp63, the downstream transcription factor in the regulatory circuit decrease leading to a molecular phenotype switch. It remains to be tested if modulation of the other members of this circuit leads to the same effect.

\subsection{DeltaNp63 as an activator of gene transcription}

As previously mentioned, deltaNp63 was first postulated to be a dominant negative factor for p53 and TAp63 due to its lack of transactivation domains [228]. Indeed, deltaNp63 isoforms were reported to fail in the activation of p53 responsive genes in contrast to most isoforms of TAp63. Accordingly, deltaNp63 was speculated to play an oncogenic role in contrast to TAp63 which was considered to play a more tumor suppressor role similar to p53 [488]. Consistently, knockout of deltaNp63 in cervical squamous cell carcinoma and human keratinocytes had a negative effect on cell growth and migration [489]. On the other hand, knockout of TAp63 in the pancreatic cancer cell line MIAPaCa-2 led to increased growth rate and migration. Furthermore, knockout of deltaNp63 resulted in decreased growth and colony formation in lung and esophagus squamous cell carcinomas [490]. 
In chapter 5, we report deltaNp63 as an activator of gene transcription. This is quite surprising due to the general conception that deltaNp63 is unable to activate transcription. Interestingly, our report is joined by other studies reporting the role of deltaNp63 as a transcription activator [491-496]. For example, the viral oncogene protein BamHI-A rightward frame 1 (BARF1) was shown to be exclusively transactivated by deltaNp63 and not p53 or TAp63 in epithelial tumors [492]. Moreover, NECTIN1 which we reported as a highly dependent gene in squamous pancreatic cancer, was also identified to be activated via two deltaNp63-dependent enhancers in skin [493]. The mechanism by which this activation occurs is still unclear and needs further investigation.

It should be noted that in both cell lines that we identified as squamous, p53 was either mutated (in the case of BxPC3) or null (L3.6pl). Bailey et al. also identified mutation of p53 as one of the characteristics of the squamous subtype [215]. So a dominant negative effect for p53 is less probable in this case with deltaNp63 able to activate genes on its own. Notably, the antiapoptotic effects of deltaNp63 were observed even in the case of p53 mutation [497]. A rescue experiment after knockdown or knockout of deltaNp63 with constructs including and lacking the transactivation domains would confirm if TAp63 is able to activate the target genes of deltaNp63. Other constructs with mutant deltaNp63 at different site can identify the region associated with the activation of genes. Additionally, a study of the interactome of deltaNp63 can also uncover cooperating factors that can be recruited and lead to the activation of target genes.

Additionally, we observed that only a subset of pancreatic cancer cells express deltaNp63 (L3.6pl, BxPC3, and moderately DanG) while MIAPaca-2 expresses TAp63. The mutual exclusivity of p63 isoform expression in pancreatic cancer cells is of high interest. Nonoverlapping patterns of expression for the different isoforms has already been reported in a 
panel of cell lines with each isoform interacting with a distinct set of transcription factors [498]. In triple negative breast cancer, both deltaNp63 and TAp63 are expressed with the latter associated with better prognosis [234]. Investigating the occupancy profile of TAp63 in MIAPaca-2 and comparing it with the H3K27ac profiles in MIAPaca-2 and deltaNp63 occupancy in BxPC3 and L3.6pl can shed light on the role of TAp63 in pancreatic cancer. This can also be combined with studying the gene expression profile upon loss of TAp63 in MIAPaca-2. Overexpression of deltaNp63 and TAp63 followed by evaluating proliferation, aggressiveness and gene expression can further elucidate the different roles of these isoforms in pancreatic cancer. However, it should be taken into consideration that overexpression of those two isoforms in deficient cell systems might not give an accurate representation for the real role of these isoforms. This is due to the absence of supporting networks of specific transcription factors possibly mediating the effects of p63 in normal conditions.

Finally, to further validate the dependence of enhancers and gene activation on deltaNp63, tethering of the two isoforms to the active enhancer regions that we identified can verify the dependence of these enhancers on deltaNp63. Tethering can be performed by utilizing guide RNAs to recruit dCas9 fusion proteins with deltaNp63 to these exclusive regions $[499,500]$. This can also be extended to the tethering of TAp63 and mutated forms of deltaNp63 to further investigate the effects of different isoforms in gene activation. Future studies will uncover the mechanisms and dependencies of deltaNp63 in a broader and more precise manner.

\subsection{Epigenetic approaches in precision-based medicine}

Recent advances in molecular subtyping of pancreatic cancer patients uncovered that using gene expression profiles in contrast to mutational landscapes is a robust approach for classification of distinct subtypes. Consistently, we reported in chapter 5 that both BxPC-3 and 
L3.6pl cells represent the squamous molecular subtype. Notably, BxPC-3 pancreatic cancer cell line is devoid of KRAS mutations which is highly uncommon in pancreatic cancer [501]. In spite of this disparity, both BxPC-3 and L3.6pl recapitulated the same molecular subtype and shared highly common features concerning their gene expression and epigenetic profiles. Such a phenomenon can support a paradigm of molecular classification of diverse malignancies that may be heterogeneous in mutations but share common features due to shared active regulatory transcriptional factors circuitry. We speculate that this may extend to cancers from various types which share common activators. In the near future, a new precision-based approach can disregard classical attributes of a certain cancer and rather try to identify specific activators for pre-defined gene expression profiles such as super enhancers and active transcription factors which can be specifically targeted and perturbed.

On the other hand, a uniform molecular classification of pancreatic cancer can serve in the accelerated application of these approaches in the clinic. A new update for the classification without arriving to a general consensus hinders the efforts to most effectively use and apply those methods. Given the plethora of high throughput sequencing and general diverse analyses, a committee to critically appraise these classifications and translate these findings to the general medical and scientific community will be highly beneficial.

In this work, we have consistently showed the high potential of epigenetic mechanisms to be leveraged in precision-based medicine approaches. In chapter 3, we proposed a mechanism underlying the synergy of BET and HDAC inhibitors that target the activation of aberrant transcriptional activation brought by the transcription factor FOXA1. Accordingly, cancers where FOXA1 plays a significant role can benefit from treatment with both of these epigenetic modulators. In chapter 4, we identified deltaNp63 as a major driver in the squamous subtype by activating super enhancers. Thus, this specific subtype of patients who characteristically show 
poorer prognosis can potentially benefit from inhibitors that target super enhancers such as THZ1 or agents that affect the stability of deltaNp63 like HDAC inhibitors [452, 471, 502]. Additionally, we reported a sensitizing potential for BET inhibitors in pancreatic cancer cells in chapter 5 . The eRNA for the active enhancers that we identified in paclitaxel-resistant cells have the potential to be used to identify subgroups of patients that might be resistant to paclitaxel and responsive to BET sensitization. Altogether, we showed various epigenetic approaches that have the potential to be successfully applied in the new era of precision-based medicine for cancer patients.

\subsection{BET inhibitors as eminent epigenetic modulators in cancer}

BET inhibitors role in targeting distal regulatory elements was thoroughly discussed in Chapter 3. A main focus of this project was to study the modulation of gene expression regulation by inhibiting the epigenetic reader family of BET proteins. As previously mentioned, while BET inhibitors are heavily studied, their mechanism of action persists to be poorly understood [211]. In chapter 5, we propose a dose-dependent effect for the BET inhibitor, JQ1, where low concentrations have specific effects on BET-dependent genes and higher concentrations lead to cell cycle arrest and a more general effect on gene transcription. We proposed in the discussion part of chapter 5, various hypotheses that can underlie this dose-dependence (Figure 31). One of those theories included a bivalent binding pattern which can lead to sensitivity to high concentration when both bromodomain pockets need to bind to acetylated histones at a certain region. This theory can be tested by using highly selective BD1 and BD2 inhibitors which target only one of the bromodomains. If such a hypothesis is true, this will endow BD1 and BD2 inhibitors with high context-specificity due to various dependencies on BD1 and BD2 in different systems. This can be investigated by testing specific and general BET inhibitors in different 
systems to check their specificity. Indeed, BD2-specific inhibitors have shown efficacy by affecting proliferation in only few systems [348, 503].

Till this date, targeting of BETs was limited to targeting the bromodomain- dependent effects. As new modulators that affect the stability of the BET family (discussed in chapter 3) showed more profound effects, non-bromodomain effects also seem to play an important role and can in theory be selectively targeted [504]. Notably, the extra-terminal domain can interact with many factors with similar and unique interactors for each family member [262]. Targeting these interactions or the pathways they activate can also be specific and effective targets.

\subsection{Concluding remarks}

In this project, we have studied different patterns of gene transcriptional activation via distal regulatory elements in the context of cancer. Aberrant gene transcriptional regulation is one of the characteristics of malignancy which can be most efficiently and specifically manipulated through enhancer elements. A bank of information for activated enhancers or super enhancers interconnected with dependencies and biomarkers may significantly play a role in optimization of therapy for patients suffering from cancer and other diseases alike. 


\section{References}

1. Allis, C.D. and T. Jenuwein, The molecular hallmarks of epigenetic control. Nat Rev Genet, 2016. 17(8): p. 487-500.

2. Gold, M., J. Hurwitz, and M. Anders, THE ENZYMATIC METHYLATION OF RNA AND DNA, II. ON THE SPECIES SPECIFICITY OF THE METHYLATION ENZYMES. Proc Natl Acad Sci U S A, 1963. 50(1): p. 164-9.

3. Venner, $\mathrm{H}$. and $\mathrm{H}$. Reinert, Possible role of methylated DNA bases for the transcription of the genetic information. Z Allg Mikrobiol, 1973. 13(7): p. 613-24.

4. Razin, A. and A.D. Riggs, DNA methylation and gene function. Science, 1980. 210(4470): p. 60410.

5. Quina, A.S., M. Buschbeck, and L. Di Croce, Chromatin structure and epigenetics. Biochem Pharmacol, 2006. 72(11): p. 1563-9.

6. Tie, F., et al., CBP-mediated acetylation of histone H3 lysine 27 antagonizes Drosophila Polycomb silencing. Development, 2009. 136(18): p. 3131-41.

7. Pavri, R., et al., Histone H2B monoubiquitination functions cooperatively with FACT to regulate elongation by RNA polymerase II. Cell, 2006. 125(4): p. 703-17.

8. Bell, O., et al., Localized H3K36 methylation states define histone H4K16 acetylation during transcriptional elongation in Drosophila. Embo j, 2007. 26(24): p. 4974-84.

9. Brinkman, A.B., et al., Histone modification patterns associated with the human $X$ chromosome. EMBO Rep, 2006. 7(6): p. 628-34.

10. Simic, R., et al., Chromatin remodeling protein Chd1 interacts with transcription elongation factors and localizes to transcribed genes. Embo j, 2003. 22(8): p. 1846-56.

11. Winston, F. and M. Carlson, Yeast SNF/SWI transcriptional activators and the SPT/SIN chromatin connection. Trends Genet, 1992. 8(11): p. 387-91.

12. Delmas, V., D.G. Stokes, and R.P. Perry, A mammalian DNA-binding protein that contains a chromodomain and an SNF2/SWI2-like helicase domain. Proc Natl Acad Sci U S A, 1993. 90(6): p. 2414-8.

13. Varga-Weisz, P.D., T.A. Blank, and P.B. Becker, Energy-dependent chromatin accessibility and nucleosome mobility in a cell-free system. Embo j, 1995. 14(10): p. 2209-16.

14. Gupta, R.A., et al., Long non-coding RNA HOTAIR reprograms chromatin state to promote cancer metastasis. Nature, 2010. 464(7291): p. 1071-6.

15. Salido, E.C., et al., Expression of the X-inactivation-associated gene XIST during spermatogenesis. Nat Genet, 1992. 2(3): p. 196-9. 
16. McCarrey, J.R. and D.D. Dilworth, Expression of Xist in mouse germ cells correlates with $X$ chromosome inactivation. Nat Genet, 1992. 2(3): p. 200-3.

17. Brockdorff, N., et al., The product of the mouse Xist gene is a $15 \mathrm{~kb}$ inactive $X$-specific transcript containing no conserved ORF and located in the nucleus. Cell, 1992. 71(3): p. 515-26.

18. Zhang, T., S. Cooper, and N. Brockdorff, The interplay of histone modifications - writers that read. EMBO Rep, 2015. 16(11): p. 1467-81.

19. Allfrey, V.G., R. Faulkner, and A.E. Mirsky, ACETYLATION AND METHYLATION OF HISTONES AND THEIR POSSIBLE ROLE IN THE REGULATION OF RNA SYNTHESIS. Proc Natl Acad Sci U S A, 1964. 51: p. 786-94.

20. Kim, S.C., et al., Substrate and functional diversity of lysine acetylation revealed by a proteomics survey. Mol Cell, 2006. 23(4): p. 607-18.

21. Xu, Y.M., J.Y. Du, and A.T. Lau, Posttranslational modifications of human histone H3: an update. Proteomics, 2014. 14(17-18): p. 2047-60.

22. McGhee, J.D., et al., Histone hyperacetylation has little effect on the higher order folding of chromatin. Nucleic Acids Res, 1983. 11(12): p. 4065-75.

23. Protacio, R.U., et al., Effects of histone tail domains on the rate of transcriptional elongation through a nucleosome. Mol Cell Biol, 2000. 20(23): p. 8866-78.

24. Wang, X., et al., Acetylation increases the alpha-helical content of the histone tails of the nucleosome. J Biol Chem, 2000. 275(45): p. 35013-20.

25. Strahl, B.D. and C.D. Allis, The language of covalent histone modifications. Nature, 2000. 403(6765): p. 41-5.

26. Dhalluin, C., et al., Structure and ligand of a histone acetyltransferase bromodomain. Nature, 1999. 399(6735): p. 491-6.

27. Karlic, R., et al., Histone modification levels are predictive for gene expression. Proc Natl Acad Sci U S A, 2010. 107(7): p. 2926-31.

28. Wilkins, B.J., et al., Genetically encoding lysine modifications on histone H4. ACS Chem Biol, 2015. 10(4): p. 939-44.

29. Kimura, H., Histone modifications for human epigenome analysis. J Hum Genet, 2013. 58(7): p. 439-45.

30. Rothbart, S.B. and B.D. Strahl, Interpreting the language of histone and DNA modifications. Biochim Biophys Acta, 2014. 1839(8): p. 627-43.

31. Pokholok, D.K., et al., Genome-wide map of nucleosome acetylation and methylation in yeast. Cell, 2005. 122(4): p. 517-27.

32. Minsky, N., et al., Monoubiquitinated H2B is associated with the transcribed region of highly expressed genes in human cells. Nat Cell Biol, 2008. 10(4): p. 483-8. 
33. Sawan, C. and Z. Herceg, Histone modifications and cancer. Adv Genet, 2010. 70: p. 57-85.

34. Clarke, S., Protein methylation. Curr Opin Cell Biol, 1993. 5(6): p. 977-83.

35. Wang, Z., et al., Combinatorial patterns of histone acetylations and methylations in the human genome. Nat Genet, 2008. 40(7): p. 897-903.

36. Young, M.D., et al., ChIP-seq analysis reveals distinct H3K27me3 profiles that correlate with transcriptional activity. Nucleic Acids Res, 2011. 39(17): p. 7415-27.

37. Arthur, R.K., et al., Evolution of H3K27me3-marked chromatin is linked to gene expression evolution and to patterns of gene duplication and diversification. Genome Res, 2014. 24(7): p. 1115-24.

38. Daujat, S., et al., H3K64 trimethylation marks heterochromatin and is dynamically remodeled during developmental reprogramming. Nat Struct Mol Biol, 2009. 16(7): p. 777-81.

39. Snowden, A.W., et al., Gene-specific targeting of H3K9 methylation is sufficient for initiating repression in vivo. Curr Biol, 2002. 12(24): p. 2159-66.

40. Mermoud, J.E., et al., Histone $H 3$ lysine 9 methylation occurs rapidly at the onset of random $X$ chromosome inactivation. Curr Biol, 2002. 12(3): p. 247-51.

41. Ringrose, L., H. Ehret, and R. Paro, Distinct contributions of histone H3 lysine 9 and 27 methylation to locus-specific stability of polycomb complexes. Mol Cell, 2004. 16(4): p. 641-53.

42. Kaimori, J.Y., et al., Histone $\mathrm{H} 4$ lysine 20 acetylation is associated with gene repression in human cells. Sci Rep, 2016. 6: p. 24318.

43. Weake, V.M. and J.L. Workman, Histone ubiquitination: triggering gene activity. Mol Cell, 2008. 29(6): p. 653-63.

44. Zhou, W., et al., Histone H2A monoubiquitination represses transcription by inhibiting RNA polymerase II transcriptional elongation. Mol Cell, 2008. 29(1): p. 69-80.

45. Jahan, S. and J.R. Davie, Protein arginine methyltransferases (PRMTs): role in chromatin organization. Adv Biol Regul, 2015. 57: p. 173-84.

46. Weiss, V.H., et al., The structure and oligomerization of the yeast arginine methyltransferase, Hmt1. Nat Struct Biol, 2000. 7(12): p. 1165-71.

47. Jenuwein, T., et al., SET domain proteins modulate chromatin domains in eu- and heterochromatin. Cell Mol Life Sci, 1998. 54(1): p. 80-93.

48. Sze, C.C. and A. Shilatifard, MLL3/MLL4/COMPASS Family on Epigenetic Regulation of Enhancer Function and Cancer. Cold Spring Harb Perspect Med, 2016. 6(11).

49. Margueron, R. and D. Reinberg, The Polycomb complex PRC2 and its mark in life. Nature, 2011. 469(7330): p. 343-9. 
50. Shiloh, Y., et al., RNF20-RNF40: A ubiquitin-driven link between gene expression and the DNA damage response. FEBS Lett, 2011. 585(18): p. 2795-802.

51. Torres, I.O. and D.G. Fujimori, Functional coupling between writers, erasers and readers of histone and DNA methylation. Curr Opin Struct Biol, 2015. 35: p. 68-75.

52. Seto, E. and M. Yoshida, Erasers of histone acetylation: the histone deacetylase enzymes. Cold Spring Harb Perspect Biol, 2014. 6(4): p. a018713.

53. Shi, Y., et al., Histone demethylation mediated by the nuclear amine oxidase homolog LSD1. Cell, 2004. 119(7): p. 941-53.

54. Shi, Y. and J.R. Whetstine, Dynamic regulation of histone lysine methylation by demethylases. Mol Cell, 2007. 25(1): p. 1-14.

55. Musselman, C.A., et al., Perceiving the epigenetic landscape through histone readers. Nat Struct Mol Biol, 2012. 19(12): p. 1218-27.

56. Maurer-Stroh, S., et al., The Tudor domain 'Royal Family': Tudor, plant Agenet, Chromo, PWWP and MBT domains. Trends Biochem Sci, 2003. 28(2): p. 69-74.

57. $\mathrm{Li}, \mathrm{H}$., et al., Molecular basis for site-specific read-out of histone H3K4me3 by the BPTF PHD finger of NURF. Nature, 2006. 442(7098): p. 91-5.

58. Lee, J.S., E. Smith, and A. Shilatifard, The language of histone crosstalk. Cell, 2010. 142(5): $p$. 682-5.

59. Roeder, R.G., The role of general initiation factors in transcription by RNA polymerase II. Trends Biochem Sci, 1996. 21(9): p. 327-35.

60. Heintzman, N.D., et al., Distinct and predictive chromatin signatures of transcriptional promoters and enhancers in the human genome. Nat Genet, 2007. 39(3): p. 311-8.

61. Ong, C.T. and V.G. Corces, Enhancer function: new insights into the regulation of tissue-specific gene expression. Nat Rev Genet, 2011. 12(4): p. 283-93.

62. Liang, G., et al., Distinct localization of histone $\mathrm{H3}$ acetylation and H3-K4 methylation to the transcription start sites in the human genome. Proc Natl Acad Sci U S A, 2004. 101(19): p. 735762.

63. Barbieri, M., et al., Active and poised promoter states drive folding of the extended HoxB locus in mouse embryonic stem cells. Nat Struct Mol Biol, 2017. 24(6): p. 515-524.

64. Creyghton, M.P., et al., Histone H3K27ac separates active from poised enhancers and predicts developmental state. Proc Natl Acad Sci U S A, 2010. 107(50): p. 21931-6.

65. Bernhart, S.H., et al., Changes of bivalent chromatin coincide with increased expression of developmental genes in cancer. Sci Rep, 2016. 6: p. 37393.

66. Ko, J.Y., S. Oh, and K.H. Yoo, Functional Enhancers As Master Regulators of Tissue-Specific Gene Regulation and Cancer Development. Mol Cells, 2017. 40(3): p. 169-177. 
67. Kaiser, V.B. and C.A. Semple, When TADs go bad: chromatin structure and nuclear organisation in human disease. F1000Res, 2017. 6.

68. Mutskov, V.J., et al., The barrier function of an insulator couples high histone acetylation levels with specific protection of promoter DNA from methylation. Genes Dev, 2002. 16(12): p. 1540-54.

69. Weth, O., et al., CTCF induces histone variant incorporation, erases the H3K27me3 histone mark and opens chromatin. Nucleic Acids Res, 2014. 42(19): p. 11941-51.

70. Buratowski, S., Progression through the RNA polymerase I/ CTD cycle. Mol Cell, 2009. 36(4): $\mathrm{p}$. 541-6.

71. Kanno, T., et al., BRD4 assists elongation of both coding and enhancer RNAs by interacting with acetylated histones. Nat Struct Mol Biol, 2014. 21(12): p. 1047-57.

72. Scheidegger, A. and S. Nechaev, RNA polymerase II pausing as a context-dependent reader of the genome. Biochem Cell Biol, 2016. 94(1): p. 82-92.

73. Yamaguchi, Y., H. Shibata, and H. Handa, Transcription elongation factors DSIF and NELF: promoter-proximal pausing and beyond. Biochim Biophys Acta, 2013. 1: p. 98-104.

74. Kwak, H. and J.T. Lis, Control of transcriptional elongation. Annu Rev Genet, 2013. 47: p. 483508.

75. Nechaev, S. and K. Adelman, Pol I/ waiting in the starting gates: Regulating the transition from transcription initiation into productive elongation. Biochim Biophys Acta, 2011. 1: p. 34-45.

76. Marshall, N.F. and D.H. Price, Purification of P-TEFb, a transcription factor required for the transition into productive elongation. J Biol Chem, 1995. 270(21): p. 12335-8.

77. Washburn, R.S. and M.E. Gottesman, Regulation of transcription elongation and termination. Biomolecules, 2015. 5(2): p. 1063-78.

78. Allen, B.L. and D.J. Taatjes, The Mediator complex: a central integrator of transcription. Nat Rev Mol Cell Biol, 2015. 16(3): p. 155-66.

79. Schilbach, S., et al., Structures of transcription pre-initiation complex with TFIIH and Mediator. Nature, 2017. 551(7679): p. 204-209.

80. Kornberg, R.D., Mediator and the mechanism of transcriptional activation. Trends Biochem Sci, 2005. 30(5): p. 235-9.

81. Poss, Z.C., C.C. Ebmeier, and D.J. Taatjes, The Mediator complex and transcription regulation. Crit Rev Biochem Mol Biol, 2013. 48(6): p. 575-608.

82. Kagey, M.H., et al., Mediator and cohesin connect gene expression and chromatin architecture. Nature, 2010. 467(7314): p. 430-5.

83. Dunn, T.M., et al., An operator at -280 base pairs that is required for repression of araBAD operon promoter: addition of DNA helical turns between the operator and promoter cyclically hinders repression. Proc Natl Acad Sci U S A, 1984. 81(16): p. 5017-20. 
84. Wendt, K.S., et al., Cohesin mediates transcriptional insulation by CCCTC-binding factor. Nature, 2008. 451(7180): p. 796-801.

85. Bell, A.C., A.G. West, and G. Felsenfeld, The protein CTCF is required for the enhancer blocking activity of vertebrate insulators. Cell, 1999. 98(3): p. 387-96.

86. Liu, J., et al., Transcriptional dysregulation in NIPBL and cohesin mutant human cells. PLoS Biol, 2009. 7(5): p. e1000119.

87. Lewis, B.A. and D. Reinberg, The mediator coactivator complex: functional and physical roles in transcriptional regulation. J Cell Sci, 2003. 116(Pt 18): p. 3667-75.

88. Petrenko, N., et al., Mediator Undergoes a Compositional Change during Transcriptional Activation. Mol Cell, 2016. 64(3): p. 443-454.

89. Spitz, F. and E.E. Furlong, Transcription factors: from enhancer binding to developmental control. Nat Rev Genet, 2012. 13(9): p. 613-26.

90. Grosschedl, R. and M.L. Birnstiel, Spacer DNA sequences upstream of the T-A-T-A-A-A-T-A sequence are essential for promotion of H2A histone gene transcription in vivo. Proc Natl Acad Sci U S A, 1980. 77(12): p. 7102-6.

91. Laimins, L.A., et al., Host-specific activation of transcription by tandem repeats from simian virus 40 and Moloney murine sarcoma virus. Proc Natl Acad Sci U S A, 1982. 79(21): p. 6453-7.

92. Rabbitts, T.H., et al., Transcription enhancer identified near the human $C$ mu immunoglobulin heavy chain gene is unavailable to the translocated c-myc gene in a Burkitt lymphoma. Nature, 1983. 306(5945): p. 806-9.

93. Gillies, S.D., et al., A tissue-specific transcription enhancer element is located in the major intron of a rearranged immunoglobulin heavy chain gene. Cell, 1983. 33(3): p. 717-28.

94. Toohey, M.G., K.L. Morley, and D.O. Peterson, Multiple hormone-inducible enhancers as mediators of differential transcription. Mol Cell Biol, 1986. 6(12): p. 4526-38.

95. Palstra, R.J. and F. Grosveld, Transcription factor binding at enhancers: shaping a genomic regulatory landscape in flux. Front Genet, 2012. 3: p. 195.

96. Lambert, S.A., et al., The Human Transcription Factors. Cell, 2018. 172(4): p. 650-665.

97. Zaret, K.S. and J.S. Carroll, Pioneer transcription factors: establishing competence for gene expression. Genes Dev, 2011. 25(21): p. 2227-41.

98. Yamamizu, K., et al., Identification of transcription factors for lineage-specific ESC differentiation. Stem Cell Reports, 2013. 1(6): p. 545-59.

99. Najafova, Z., et al., BRD4 localization to lineage-specific enhancers is associated with a distinct transcription factor repertoire. Nucleic Acids Res, 2017. 45(1): p. 127-141.

100. Lan, X., et al., Integration of Hi-C and ChIP-seq data reveals distinct types of chromatin linkages. Nucleic Acids Res, 2012. 40(16): p. 7690-704. 
101. Grossman, S.R., et al., Positional specificity of different transcription factor classes within enhancers. Proc Natl Acad Sci U S A, 2018. 115(30): p. E7222-e7230.

102. Mayran, A. and J. Drouin, Pioneer transcription factors shape the epigenetic landscape. J Biol Chem, 2018.

103. Kim, T.K., et al., Widespread transcription at neuronal activity-regulated enhancers. Nature, 2010. 465(7295): p. $182-7$.

104. Schaukowitch, K., et al., Enhancer RNA facilitates NELF release from immediate early genes. Mol Cell, 2014. 56(1): p. 29-42.

105. Liang, J., et al., Epstein-Barr virus super-enhancer eRNAs are essential for MYC oncogene expression and lymphoblast proliferation. Proc Natl Acad Sci U S A, 2016. 113(49): p. 1412114126.

106. Pnueli, L., et al., RNA transcribed from a distal enhancer is required for activating the chromatin at the promoter of the gonadotropin alpha-subunit gene. Proc Natl Acad Sci U S A, 2015. 112(14): p. 4369-74.

107. Kim, Y.W., et al., Chromatin looping and eRNA transcription precede the transcriptional activation of gene in the beta-globin locus. Biosci Rep, 2015. 35(2).

108. Leveille, N., et al., Genome-wide profiling of p53-regulated enhancer RNAs uncovers a subset of enhancers controlled by a IncRNA. Nat Commun, 2015. 6: p. 6520.

109. Core, L.J., J.J. Waterfall, and J.T. Lis, Nascent RNA sequencing reveals widespread pausing and divergent initiation at human promoters. Science, 2008. 322(5909): p. 1845-8.

110. Schwalb, B., et al., TT-seq maps the human transient transcriptome. Science, 2016. 352(6290): p. $1225-8$.

111. Mahat, D.B., et al., Base-pair-resolution genome-wide mapping of active RNA polymerases using precision nuclear run-on (PRO-seq). Nat Protoc, 2016. 11(8): p. 1455-76.

112. Chu, T., et al., Chromatin run-on reveals the transcriptional etiology of glioblastoma multiforme. bioRxiv, 2018.

113. Benabdallah, N.S. and W.A. Bickmore, Regulatory Domains and Their Mechanisms. Cold Spring Harb Symp Quant Biol, 2015. 80: p. 45-51.

114. He, B., et al., Global view of enhancer-promoter interactome in human cells. Proc Natl Acad Sci U $S$ A, 2014. 111(21): p. E2191-9.

115. Dekker, J., et al., Capturing chromosome conformation. Science, 2002. 295(5558): p. 1306-11.

116. van Berkum, N.L., et al., Hi-C: a method to study the three-dimensional architecture of genomes. $J$ Vis Exp, 2010(39).

117. Fullwood, M.J., et al., An oestrogen-receptor-alpha-bound human chromatin interactome. Nature, 2009. 462(7269): p. 58-64. 
118. Mumbach, M.R., et al., HiChIP: efficient and sensitive analysis of protein-directed genome architecture. Nat Methods, 2016. 13(11): p. 919-922.

119. Pope, B.D., et al., Topologically associating domains are stable units of replication-timing regulation. Nature, 2014. 515(7527): p. 402-5.

120. Ong, C.T. and V.G. Corces, CTCF: an architectural protein bridging genome topology and function. Nat Rev Genet, 2014. 15(4): p. 234-46.

121. Schmitt, A.D., et al., A Compendium of Chromatin Contact Maps Reveals Spatially Active Regions in the Human Genome. Cell Rep, 2016. 17(8): p. 2042-2059.

122. Nora, E.P., et al., Targeted Degradation of CTCF Decouples Local Insulation of Chromosome Domains from Genomic Compartmentalization. Cell, 2017. 169(5): p. 930-944.e22.

123. Loven, J., et al., Selective inhibition of tumor oncogenes by disruption of super-enhancers. Cell, 2013. 153(2): p. 320-34.

124. Whyte, W.A., et al., Master transcription factors and mediator establish super-enhancers at key cell identity genes. Cell, 2013. 153(2): p. 307-19.

125. Zhang, X., et al., Identification of focally amplified lineage-specific super-enhancers in human epithelial cancers. Nat Genet, 2016. 48(2): p. 176-82.

126. Bojcsuk, D., G. Nagy, and B.L. Balint, Inducible super-enhancers are organized based on canonical signal-specific transcription factor binding elements. Nucleic Acids Res, 2017. 45(7): p. 3693-3706.

127. Gérard, D., et al., Temporal epigenomic profiling identifies AHR as dynamic super-enhancer controlled regulator of mesenchymal multipotency. bioRxiv, 2017.

128. Perez-Rico, Y.A., et al., Comparative analyses of super-enhancers reveal conserved elements in vertebrate genomes. Genome Res, 2017. 27(2): p. 259-268.

129. Sabari, B.R., et al., Coactivator condensation at super-enhancers links phase separation and gene control. Science, 2018. 361(6400).

130. Madani Tonekaboni, S.A., et al., CREAM: Clustering of genomic REgions Analysis Method. bioRxiv, 2018.

131. Hnisz, D., et al., Super-enhancers in the control of cell identity and disease. Cell, 2013. 155(4): $p$. 934-47.

132. Vahedi, G., et al., Super-enhancers delineate disease-associated regulatory nodes in $T$ cells. Nature, 2015. 520(7548): p. 558-62.

133. Sun, W., et al., Integrative analysis of super enhancer SNPs for type 2 diabetes. PLoS One, 2018. 13(1): p. e0192105.

134. Corradin, O. and P.C. Scacheri, Enhancer variants: evaluating functions in common disease. Genome Med, 2014. 6(10): p. 85. 
135. Smith, E. and A. Shilatifard, Enhancer biology and enhanceropathies. Nature Structural \&Amp; Molecular Biology, 2014. 21: p. 210.

136. Groschel, S., et al., A single oncogenic enhancer rearrangement causes concomitant EVI1 and GATA2 deregulation in leukemia. Cell, 2014. 157(2): p. 369-381.

137. Knoechel, B., et al., An epigenetic mechanism of resistance to targeted therapy in $T$ cell acute lymphoblastic leukemia. Nat Genet, 2014. 46(4): p. 364-70.

138. Heyn, H., et al., Epigenomic analysis detects aberrant super-enhancer DNA methylation in human cancer. Genome Biol, 2016. 17: p. 11.

139. Takeda, D.Y., et al., A Somatically Acquired Enhancer of the Androgen Receptor Is a Noncoding Driver in Advanced Prostate Cancer. Cell, 2018. 174(2): p. 422-432.e13.

140. Zeid, R., et al., Enhancer invasion shapes MYCN-dependent transcriptional amplification in neuroblastoma. Nat Genet, 2018. 50(4): p. 515-523.

141. Roe, J.S., et al., Enhancer Reprogramming Promotes Pancreatic Cancer Metastasis. Cell, 2017. 170(5): p. 875-888.e20.

142. Suzuki, A., et al., Aberrant transcriptional regulations in cancers: genome, transcriptome and epigenome analysis of lung adenocarcinoma cell lines. Nucleic Acids Res, 2014. 42(22): p. 13557-72.

143. Jemal, A., et al., Global cancer statistics. CA Cancer J Clin, 2011. 61(2): p. 69-90.

144. Torre, L.A., et al., Global Cancer Incidence and Mortality Rates and Trends-An Update. Cancer Epidemiol Biomarkers Prev, 2016. 25(1): p. 16-27.

145. Puhalla, S., S. Bhattacharya, and N.E. Davidson, Hormonal therapy in breast cancer: a model disease for the personalization of cancer care. Mol Oncol, 2012. 6(2): p. 222-36.

146. Lumachi, F., et al., Treatment of estrogen receptor-positive breast cancer. Curr Med Chem, 2013. 20(5): p. 596-604.

147. Yasar, P., et al., Molecular mechanism of estrogen-estrogen receptor signaling. Reprod Med Biol, 2017. 16(1): p. 4-20.

148. Losel, R. and M. Wehling, Nongenomic actions of steroid hormones. Nat Rev Mol Cell Biol, 2003. 4(1): p. 46-56.

149. Carroll, J.S., et al., Genome-wide analysis of estrogen receptor binding sites. Nature Genetics, 2006. 38: p. 1289.

150. Carleton, J.B., K.C. Berrett, and J. Gertz, Multiplex Enhancer Interference Reveals Collaborative Control of Gene Regulation by Estrogen Receptor alpha-Bound Enhancers. Cell Syst, 2017. 5(4): p. 333-344.e5.

151. Murakami, S., A. Nagari, and W.L. Kraus, Dynamic assembly and activation of estrogen receptor alpha enhancers through coregulator switching. Genes Dev, 2017. 31(15): p. 1535-1548. 
152. Nagarajan, S., et al., Bromodomain protein BRD4 is required for estrogen receptor-dependent enhancer activation and gene transcription. Cell Rep, 2014. 8(2): p. 460-9.

153. Malvezzi, M., et al., European cancer mortality predictions for the year 2017, with focus on lung cancer. Ann Oncol, 2017. 28(5): p. 1117-1123.

154. Roe, J.S., et al., Enhancer Reprogramming Promotes Pancreatic Cancer Metastasis. Cell, 2017. 170(5): p. 875-888.

155. Chandra, R. and R.A. Liddle, Modulation of pancreatic exocrine and endocrine secretion. Curr Opin Gastroenterol, 2013. 29(5): p. 517-22.

156. Liang, C., et al., Complex roles of the stroma in the intrinsic resistance to gemcitabine in pancreatic cancer: where we are and where we are going. Experimental \&Amp; Molecular Medicine, 2017. 49: p. e406.

157. Koorstra, J.B., et al., Morphogenesis of pancreatic cancer: role of pancreatic intraepithelial neoplasia (PanINs). Langenbecks Arch Surg, 2008. 393(4): p. 561-70.

158. Distler, M., et al., Precursor lesions for sporadic pancreatic cancer: PanIN, IPMN, and MCN. Biomed Res Int, 2014. 2014: p. 474905.

159. Hruban, R.H., et al., Progression model for pancreatic cancer. Clin Cancer Res, 2000. 6(8): p. 2969-72.

160. Lemstrova, R., et al., Dysregulation of KRAS signaling in pancreatic cancer is not associated with KRAS mutations and outcome. Oncol Lett, 2017. 14(5): p. 5980-5988.

161. di Magliano, M.P. and C.D. Logsdon, Roles for KRAS in pancreatic tumor development and progression. Gastroenterology, 2013. 144(6): p. 1220-9.

162. McWilliams, R.R., et al., Prevalence of CDKN2A mutations in pancreatic cancer patients: implications for genetic counseling. Eur J Hum Genet, 2011. 19(4): p. 472-8.

163. Lukas, J., et al., Retinoblastoma-protein-dependent cell-cycle inhibition by the tumour suppressor p16. Nature, 1995. 375(6531): p. 503-6.

164. Weissmueller, S., et al., Mutant p53 drives pancreatic cancer metastasis through cellautonomous PDGF receptor beta signaling. Cell, 2014. 157(2): p. 382-394.

165. Klump, B., et al., Methylation status of p14ARF and p16INK4a as detected in pancreatic secretions. Br J Cancer, 2003. 88(2): p. 217-22.

166. Soufi, A., et al., Pioneer Transcription Factors Target Partial DNA Motifs on Nucleosomes to Initiate Reprogramming. Cell, 2015. 161(3): p. 555-568.

167. Wei, D., et al., KLF4 Is Essential for Induction of Cellular Identity Change and Acinar-to-Ductal Reprogramming during Early Pancreatic Carcinogenesis. Cancer Cell, 2016. 29(3): p. 324-338. 
168. Patra, K.C., N. Bardeesy, and Y. Mizukami, Diversity of Precursor Lesions For Pancreatic Cancer: The Genetics and Biology of Intraductal Papillary Mucinous Neoplasm. Clinical And Translational Gastroenterology, 2017. 8: p. e86.

169. Hariharan, D., A. Saied, and H.M. Kocher, Analysis of mortality rates for pancreatic cancer across the world. HPB, 2008. 10(1): p. 58-62.

170. Beger, H.G., et al., Pancreatic cancer--low survival rates. Dtsch Arztebl Int, 2008. 105(14): p. 255-62.

171. Klein, A.P., Identifying people at a high risk of developing pancreatic cancer. Nat Rev Cancer, 2013. 13(1): p. 66-74.

172. Tempero, M.A., et al., Pancreatic adenocarcinoma, version 2.2014: featured updates to the NCCN guidelines. J Natl Compr Canc Netw, 2014. 12(8): p. 1083-93.

173. Cid-Arregui, A. and V. Juarez, Perspectives in the treatment of pancreatic adenocarcinoma. World J Gastroenterol, 2015. 21(31): p. 9297-316.

174. Lee, H.S. and S.W. Park, Systemic Chemotherapy in Advanced Pancreatic Cancer. Gut Liver, 2016. 10(3): p. 340-7.

175. Longley, D.B., D.P. Harkin, and P.G. Johnston, 5-Fluorouracil: mechanisms of action and clinical strategies. Nature Reviews Cancer, 2003. 3: p. 330.

176. Thibodeau, S. and I.A. Voutsadakis, FOLFIRINOX Chemotherapy in Metastatic Pancreatic Cancer: A Systematic Review and Meta-Analysis of Retrospective and Phase II Studies. J Clin Med, 2018. 7(1).

177. Shore, S., et al., Review article: chemotherapy for pancreatic cancer. Aliment Pharmacol Ther, 2003. 18(11-12): p. 1049-69.

178. Burris, H.A., 3rd, et al., Improvements in survival and clinical benefit with gemcitabine as first-line therapy for patients with advanced pancreas cancer: a randomized trial. J Clin Oncol, 1997. 15(6): p. 2403-13.

179. Sultana, A., et al., Meta-Analyses of Chemotherapy for Locally Advanced and Metastatic Pancreatic Cancer. 2007. 25(18): p. 2607-2615.

180. Von Hoff, D.D., et al., Gemcitabine plus nab-paclitaxel is an active regimen in patients with advanced pancreatic cancer: a phase I/II trial. J Clin Oncol, 2011. 29(34): p. 4548-54.

181. Goldstein, D., et al., nab-Paclitaxel plus gemcitabine for metastatic pancreatic cancer: long-term survival from a phase III trial. J Natl Cancer Inst, 2015. 107(2).

182. Kampan, N.C., et al., Paclitaxel and Its Evolving Role in the Management of Ovarian Cancer. Biomed Res Int, 2015. 2015: p. 413076.

183. Grasso, C., G. Jansen, and E. Giovannetti, Drug resistance in pancreatic cancer: Impact of altered energy metabolism. Crit Rev Oncol Hematol, 2017. 114: p. 139-152. 
184. Orr, G.A., et al., Mechanisms of Taxol resistance related to microtubules. Oncogene, 2002. 22: p. 7280.

185. Chand, S., et al., The Landscape of Pancreatic Cancer Therapeutic Resistance Mechanisms. Int J Biol Sci, 2016. 12(3): p. 273-82.

186. Gnanamony, M. and C.S. Gondi, Chemoresistance in pancreatic cancer: Emerging concepts. Oncol Lett, 2017. 13(4): p. 2507-2513.

187. Fiorini, C., et al., Mutant p53 stimulates chemoresistance of pancreatic adenocarcinoma cells to gemcitabine. Biochimica et Biophysica Acta (BBA) - Molecular Cell Research, 2015. 1853(1): p. 89-100.

188. Jia, Y. and J. Xie, Promising molecular mechanisms responsible for gemcitabine resistance in cancer. Genes \& Diseases, 2015. 2(4): p. 299-306.

189. Frese, K.K., et al., nab-Paclitaxel potentiates gemcitabine activity by reducing cytidine deaminase levels in a mouse model of pancreatic cancer. Cancer Discov, 2012. 2(3): p. 260-269.

190. Le Large, T., et al., Gemcitabine resistant pancreatic cancer cells are sensitive to paclitaxel treatment. Vol. 17. 2017. S42.

191. Whiteley, A., et al., A pilot, non-randomized evaluation of the safety of anakinra plus FOLFIRINOX in metastatic pancreatic ductal adenocarcinoma patients. Journal of Clinical Oncology, 2016. 34(15_suppl): p. e15750-e15750.

192. Troiani, T., et al., Targeting EGFR in pancreatic cancer treatment. Curr Drug Targets, 2012. 13(6): p. 802-10.

193. Golan, T., et al., Phase II study of olaparib for BRCAness phenotype in pancreatic cancer. Journal of Clinical Oncology, 2018. 36(4_suppl): p. 297-297.

194. Lee, H.S., et al., A novel HDAC inhibitor, CG200745, inhibits pancreatic cancer cell growth and overcomes gemcitabine resistance. Sci Rep, 2017. 7: p. 41615.

195. Arlt, A. and H. Schäfer, Investigational histone deacetylase inhibitors for treating pancreatic adenocarcinoma. Expert Opinion on Investigational Drugs, 2016. 25(11): p. 1251-1254.

196. Leal, A.S., et al., Bromodomain inhibitors, JQ1 and I-BET 762, as potential therapies for pancreatic cancer. Cancer Lett, 2017. 394: p. 76-87.

197. Yamamoto, K., et al., Stromal remodeling by the BET bromodomain inhibitor JQ1 suppresses the progression of human pancreatic cancer. Oncotarget, 2016. 9(10): p. 11129.

198. Garcia, P.L., et al., The BET bromodomain inhibitor JQ1 suppresses growth of pancreatic ductal adenocarcinoma in patient-derived xenograft models. Oncogene, 2016. 35(7): p. 833-45.

199. Shi, J. and C.R. Vakoc, The mechanisms behind the therapeutic activity of BET bromodomain inhibition. Mol Cell, 2014. 54(5): p. 728-36. 
200. Liu, X., W.L. Kraus, and X. Bai, Ready, pause, go: regulation of RNA polymerase II pausing and release by cellular signaling pathways. Trends Biochem Sci, 2015. 40(9): p. 516-25.

201. Itzen, F., et al., Brd4 activates P-TEFb for RNA polymerase I/ CTD phosphorylation. Nucleic Acids Res, 2014. 42(12): p. 7577-90.

202. Zhang, F. and X. Yu, WAC, a functional partner of RNF20/40, regulates histone H2B ubiquitination and gene transcription. Mol Cell, 2011. 41(4): p. 384-97.

203. Kim, J., S.B. Hake, and R.G. Roeder, The human homolog of yeast BRE1 functions as a transcriptional coactivator through direct activator interactions. Mol Cell, 2005. 20(5): p. 759-70.

204. Zhu, B., et al., Monoubiquitination of human histone H2B: the factors involved and their roles in HOX gene regulation. Mol Cell, 2005. 20(4): p. 601-11.

205. Filippakopoulos, P. and S. Knapp, Targeting bromodomains: epigenetic readers of lysine acetylation. Nat Rev Drug Discov, 2014. 13(5): p. 337-56.

206. Mirguet, O., et al., From ApoA1 upregulation to BET family bromodomain inhibition: discovery of I-BET151. Bioorg Med Chem Lett, 2012. 22(8): p. 2963-7.

207. Picaud, S., et al., $R V X-208$, an inhibitor of BET transcriptional regulators with selectivity for the second bromodomain. Proc Natl Acad Sci U S A, 2013. 110(49): p. 19754-9.

208. Xie, F., et al., The BET inhibitor I-BET762 inhibits pancreatic ductal adenocarcinoma cell proliferation and enhances the therapeutic effect of gemcitabine. Scientific Reports, 2018. 8(1): p. 8102.

209. Yamamoto, K., et al., Stromal remodeling by the BET bromodomain inhibitor JQ1 suppresses the progression of human pancreatic cancer. Oncotarget, 2016. 7(38): p. 61469-61484.

210. Sahai, V., et al., BET bromodomain inhibitors block growth of pancreatic cancer cells in threedimensional collagen. Mol Cancer Ther, 2014. 13(7): p. 1907-17.

211. Andrieu, G., A.C. Belkina, and G.V. Denis, Clinical trials for BET inhibitors run ahead of the science. Drug Discov Today Technol, 2016. 19: p. 45-50.

212. Andricovich, J., et al., Loss of KDM6A Activates Super-Enhancers to Induce Gender-Specific Squamous-like Pancreatic Cancer and Confers Sensitivity to BET Inhibitors. Cancer Cell, 2018. 33(3): p. 512-526.

213. Collisson, E.A., et al., Subtypes of pancreatic ductal adenocarcinoma and their differing responses to therapy. Nat Med, 2011. 17(4): p. 500-3.

214. Moffitt, R.A., et al., Virtual microdissection identifies distinct tumor- and stroma-specific subtypes of pancreatic ductal adenocarcinoma. Nat Genet, 2015. 47(10): p. 1168-78.

215. Bailey, P., et al., Genomic analyses identify molecular subtypes of pancreatic cancer. Nature, 2016. 531(7592): p. 47-52. 
216. Raphael, B.J.e.a., Integrated Genomic Characterization of Pancreatic Ductal Adenocarcinoma. Cancer Cell, 2017. 32(2): p. 185-203.

217. Torres, C. and P.J. Grippo, Pancreatic cancer subtypes: a roadmap for precision medicine. Ann Med, 2018. 50(4): p. 277-287.

218. Pishvaian, M.J. and J.R. Brody, Therapeutic Implications of Molecular Subtyping for Pancreatic Cancer. Oncology (Williston Park), 2017. 31(3): p. 159-66, 168.

219. Tiriac, H., et al., Organoid Profiling Identifies Common Responders to Chemotherapy in Pancreatic Cancer. Cancer Discov, 2018.

220. Lomberk, G., et al., Distinct epigenetic landscapes underlie the pathobiology of pancreatic cancer subtypes. Nat Commun, 2018. 9(1): p. 018-04383.

221. Kuhlmann, L., et al., Identification and Validation of Novel Subtype-Specific Protein Biomarkers in Pancreatic Ductal Adenocarcinoma. Pancreas, 2017. 46(3): p. 311-322.

222. Janky, R., et al., Prognostic relevance of molecular subtypes and master regulators in pancreatic ductal adenocarcinoma. BMC Cancer, 2016. 16: p. 632.

223. Zhao, L., H. Zhao, and H. Yan, Gene expression profiling of 1200 pancreatic ductal adenocarcinoma reveals novel subtypes. BMC Cancer, 2018. 18(1): p. 603.

224. Er, J.L., et al., Identification of inhibitors synergizing gemcitabine sensitivity in the squamous subtype of pancreatic ductal adenocarcinoma (PDAC). Apoptosis, 2018.

225. Brauswetter, D., et al., Molecular subtype specific efficacy of MEK inhibitors in pancreatic cancers. PLoS One, 2017. 12(9): p. e0185687.

226. Muckenhuber, A., et al., Pancreatic Ductal Adenocarcinoma Subtyping Using the Biomarkers Hepatocyte Nuclear Factor-1A and Cytokeratin-81 Correlates with Outcome and Treatment Response. Clin Cancer Res, 2018. 24(2): p. 351-359.

227. Soares, E. and H. Zhou, Master regulatory role of p63 in epidermal development and disease. Cell Mol Life Sci, 2018. 75(7): p. 1179-1190.

228. Yang, A., et al., p63, a p53 homolog at 3q27-29, encodes multiple products with transactivating, death-inducing, and dominant-negative activities. Mol Cell, 1998. 2(3): p. 305-16.

229. Brady, C.A. and L.D. Attardi, p53 at a glance. Journal of Cell Science, 2010. 123(15): p. 2527.

230. Mills, A.A., et al., p63 is a p53 homologue required for limb and epidermal morphogenesis. Nature, 1999. 398(6729): p. 708-13.

231. Yang, A., et al., p63 is essential for regenerative proliferation in limb, craniofacial and epithelial development. Nature, 1999. 398(6729): p. 714-8.

232. Bretz, A.C., et al., DeltaNp63 activates the Fanconi anemia DNA repair pathway and limits the efficacy of cisplatin treatment in squamous cell carcinoma. Nucleic Acids Res, 2016. 44(7): p. 3204-18. 
233. Kumakura, Y., et al., Elevated expression of DeltaNp63 in advanced esophageal squamous cell carcinoma. Cancer Sci, 2017. 108(11): p. 2149-2155.

234. Coates, P.J., et al., p63 isoforms in triple-negative breast cancer: DeltaNp63 associates with the basal phenotype whereas TAp63 associates with androgen receptor, lack of BRCA mutation, PTEN and improved survival. Virchows Arch, 2018. 472(3): p. 351-359.

235. Orzol, P., et al., DeltaNp63 regulates cell proliferation, differentiation, adhesion, and migration in the BL2 subtype of basal-like breast cancer. Tumour Biol, 2016. 37(8): p. 10133-40.

236. Mitani, Y., et al., Expression and Regulation of the $\Delta N$ and TAp63 Isoforms in Salivary Gland Tumorigenesis: Clinical and Experimental Findings. The American Journal of Pathology, 2011. 179(1): p. 391-399.

237. Zhuang, Z., et al., Interplay between DeltaNp63 and miR-138-5p regulates growth, metastasis and stemness of oral squamous cell carcinoma. Oncotarget, 2017. 8(13): p. 21954-21973.

238. Matsubara, R., et al., Increased DeltaNp63 expression is predictive of malignant transformation in oral epithelial dysplasia and poor prognosis in oral squamous cell carcinoma. Int J Oncol, 2011. 39(6): p. 1391-9.

239. Basturk, O., et al., DeltaNp63 expression in pancreas and pancreatic neoplasia. Mod Pathol, 2005. 18(9): p. 1193-8.

240. Xie, J.-J., et al., Super-Enhancer-Driven Long Non-Coding RNA LINC01503, Regulated by TP63, Is Over-Expressed and Oncogenic in Squamous Cell Carcinoma. Gastroenterology, 2018. 154(8): p. 2137-2151.e1.

241. Lin-Shiao, E., et al., KMT2D regulates p63 target enhancers to coordinate epithelial homeostasis. Genes Dev, 2018. 32(2): p. 181-193.

242. Ferri, E., C. Petosa, and C.E. McKenna, Bromodomains: Structure, function and pharmacology of inhibition. Biochem Pharmacol, 2016. 106: p. 1-18.

243. Xu, Y. and C.R. Vakoc, Targeting Cancer Cells with BET Bromodomain Inhibitors. Cold Spring Harb Perspect Med, 2017. 7(7).

244. Nicodeme, E., et al., Suppression of inflammation by a synthetic histone mimic. Nature, 2010. 468(7327): p. 1119-23.

245. Filippakopoulos, P., et al., Selective inhibition of BET bromodomains. Nature, 2010. 468(7327): $\mathrm{p}$. 1067-73.

246. Hessmann, E., et al., Epigenetic treatment of pancreatic cancer: is there a therapeutic perspective on the horizon? Gut, 2017. 66(1): p. 168-179.

247. Nagarajan, S., et al., BRD4 promotes p63 and GRHL3 expression downstream of FOXO in mammary epithelial cells. Nucleic Acids Res, 2017. 45(6): p. 3130-3145.

248. Pott, S. and J.D. Lieb, What are super-enhancers? Nat Genet, 2015. 47(1): p. 8-12. 
249. Evan, G.l., et al., Re-engineering the Pancreas Tumor Microenvironment: $A$ "Regenerative Program" Hacked. Clin Cancer Res. 2017 Apr 1;23(7):1647-1655. doi: 10.1158/1078-0432.CCR16-3275.

250. Zuber, V., et al., Bromodomain protein 4 discriminates tissue-specific super-enhancers containing disease-specific susceptibility loci in prostate and breast cancer. BMC Genomics, 2017. 18(1): p. 017-3620.

251. McCleland, M.L., et al., CCAT1 is an enhancer-templated RNA that predicts BET sensitivity in colorectal cancer. J Clin Invest, 2016. 126(2): p. 639-52.

252. Dave, K., et al., Mice deficient of Myc super-enhancer region reveal differential control mechanism between normal and pathological growth. Elife, 2017. 6(6): p. 23382.

253. Theodorou, V., et al., GATA3 acts upstream of FOXA1 in mediating ESR1 binding by shaping enhancer accessibility. Genome Res, 2013. 23(1): p. 12-22.

254. Xie, S., et al., Multiplexed Engineering and Analysis of Combinatorial Enhancer Activity in Single Cells. Mol Cell, 2017. 66(2): p. 285-299.

255. Hay, D., et al., Genetic dissection of the alpha-globin super-enhancer in vivo. Nat Genet, 2016. 48(8): p. 895-903.

256. Shin, H.Y., et al., Hierarchy within the mammary STAT5-driven Wap super-enhancer. Nat Genet, 2016. 48(8): p. 904-11.

257. McLean, C.Y., et al., GREAT improves functional interpretation of cis-regulatory regions. Nat Biotechnol, 2010. 28(5): p. 495-501.

258. He, H.H., et al., Differential DNase I hypersensitivity reveals factor-dependent chromatin dynamics. Genome Res, 2012. 22(6): p. 1015-25.

259. Xu, X., et al., A CRISPR-based approach for targeted DNA demethylation. Cell Discov, 2016. 2(16009).

260. Hilton, I.B., et al., Epigenome editing by a CRISPR-Cas9-based acetyltransferase activates genes from promoters and enhancers. Nat Biotechnol, 2015. 33(5): p. 510-7.

261. Li, W., et al., Functional roles of enhancer RNAs for oestrogen-dependent transcriptional activation. Nature, 2013. 498(7455): p. 516-20.

262. Rahman, S., et al., Single-cell profiling reveals that eRNA accumulation at enhancer-promoter loops is not required to sustain transcription. Nucleic Acids Res, 2017. 45(6): p. 3017-3030.

263. Hah, N., et al., Inflammation-sensitive super enhancers form domains of coordinately regulated enhancer RNAs. Proc Natl Acad Sci U S A, 2015. 112(3): p. 6.

264. Hah, N., et al., Enhancer transcripts mark active estrogen receptor binding sites. Genome Res, 2013. 23(8): p. 1210-23. 
265. Waddell, N., et al., Whole genomes redefine the mutational landscape of pancreatic cancer. Nature, 2015. 518(7540): p. 495-501.

266. Jakel, C., et al., Genome-wide genetic and epigenetic analyses of pancreatic acinar cell carcinomas reveal aberrations in genome stability. Nat Commun, 2017. 8(1): p. 017-01118.

267. Jia, J., et al., An integrated transcriptome and epigenome analysis identifies a novel candidate gene for pancreatic cancer. BMC Med Genomics, 2013. 6(33): p. 1755-8794.

268. McCleary-Wheeler, A.L., et al., Insights into the epigenetic mechanisms controlling pancreatic carcinogenesis. Cancer Lett, 2013. 328(2): p. 212-21.

269. Vogelstein, B. and K.W. Kinzler, The multistep nature of cancer. Trends Genet, 1993. 9(4): p. 138-41.

270. Iguchi, E., et al., Pancreatic Cancer, A Mis-interpreter of the Epigenetic Language. Yale J Biol Med, 2016. 89(4): p. 575-590.

271. Bradner, J.E., D. Hnisz, and R.A. Young, Transcriptional Addiction in Cancer. Cell, 2017. 168(4): p. $629-643$.

272. Sharma, S.V. and J. Settleman, Oncogene addiction: setting the stage for molecularly targeted cancer therapy. Genes Dev, 2007. 21(24): p. 3214-31.

273. Alekseyenko, A.A., et al., The oncogenic BRD4-NUT chromatin regulator drives aberrant transcription within large topological domains. Genes Dev, 2015. 29(14): p. 1507-23.

274. Iwahashi, S., et al., Effects of valproic acid in combination with S-1 on advanced pancreatobiliary tract cancers: clinical study phases I/II. Anticancer Res, 2014. 34(9): p. 5187-91.

275. Chan, E., et al., Phase I trial of vorinostat added to chemoradiation with capecitabine in pancreatic cancer. Radiother Oncol, 2016. 119(2): p. 312-8.

276. Abdelfatah, E., et al., Epigenetic therapy in gastrointestinal cancer: the right combination. Therap Adv Gastroenterol, 2016. 9(4): p. 560-79.

277. Lomberk, G.A., J. lovanna, and R. Urrutia, The promise of epigenomic therapeutics in pancreatic cancer. Epigenomics, 2016. 8(6): p. 831-42.

278. Neureiter, D., et al., Epigenetics and pancreatic cancer: pathophysiology and novel treatment aspects. World J Gastroenterol, 2014. 20(24): p. 7830-48.

279. Silverman, B.R. and J. Shi, Alterations of Epigenetic Regulators in Pancreatic Cancer and Their Clinical Implications. Int J Mol Sci, 2016. 17(12).

280. Quilichini, E. and C. Haumaitre, Implication of epigenetics in pancreas development and disease. Best Pract Res Clin Endocrinol Metab, 2015. 29(6): p. 883-98.

281. Klieser, E., et al., Role of histone deacetylases in pancreas: Implications for pathogenesis and therapy. World J Gastrointest Oncol, 2015. 7(12): p. 473-83. 
282. Damaskos, C., et al., Histone Deacetylase (HDAC) Inhibitors: Current Evidence for Therapeutic Activities in Pancreatic Cancer. Anticancer Res, 2015. 35(6): p. 3129-35.

283. French, C.A., Pathogenesis of NUT midline carcinoma. Annu Rev Pathol, 2012. 7: p. 247-65.

284. Al Diffalha, S., et al., NUT Midline Carcinoma: A Rare Malignancy. Cancer Control, 2017. 24(2): p. 202-206.

285. Evans, A.G., et al., Pathologic characteristics of NUT midline carcinoma arising in the mediastinum. Am J Surg Pathol, 2012. 36(8): p. 1222-7.

286. Fu, L.L., et al., Inhibition of BET bromodomains as a therapeutic strategy for cancer drug discovery. Oncotarget, 2015. 6(8): p. 5501-16.

287. Huang, Y., et al., Regulation of GLI Underlies a Role for BET Bromodomains in Pancreatic Cancer Growth and the Tumor Microenvironment. Clin Cancer Res, 2016. 22(16): p. 4259-70.

288. Sherman, M.H., et al., Stromal cues regulate the pancreatic cancer epigenome and metabolome. Proc Natl Acad Sci U S A, 2017. 114(5): p. 1129-1134.

289. Bian, B., et al., Gene expression profiling of patient-derived pancreatic cancer xenografts predicts sensitivity to the BET bromodomain inhibitor JQ1: implications for individualized medicine efforts. EMBO Mol Med, 2017. 9(4): p. 482-497.

290. Uccello, M., et al., Towards an optimal treatment algorithm for metastatic pancreatic ductal adenocarcinoma (PDA). Curr Oncol, 2018. 25(1): p. e90-e94.

291. McDonald, O.G., et al., Epigenomic reprogramming during pancreatic cancer progression links anabolic glucose metabolism to distant metastasis. Nat Genet, 2017. 49(3): p. 367-376.

292. Morrow, J.J., et al., Positively selected enhancer elements endow osteosarcoma cells with metastatic competence. Nat Med, 2018. 24(2): p. 176-185.

293. Mack, S.C., et al., Therapeutic targeting of ependymoma as informed by oncogenic enhancer profiling. Nature, 2018. 553(7686): p. 101-105.

294. Gryder, B.E., et al., PAX3-FOXO1 Establishes Myogenic Super Enhancers and Confers BET Bromodomain Vulnerability. Cancer Discov, 2017. 7(8): p. 884-899.

295. Chen, H.S., et al., BET-Inhibitors Disrupt Rad21-Dependent Conformational Control of KSHV Latency. PLoS Pathog, 2017. 13(1).

296. Cheung, K.L., et al., Distinct Roles of Brd2 and Brd4 in Potentiating the Transcriptional Program for Th17 Cell Differentiation. Mol Cell, 2017. 65(6): p. 1068-1080.

297. Hsu, S.C., et al., The BET Protein BRD2 Cooperates with CTCF to Enforce Transcriptional and Architectural Boundaries. Mol Cell, 2017. 66(1): p. 102-116.

298. Kaiser, V.B. and C.A. Semple, When TADs go bad: chromatin structure and nuclear organisation in human disease. F1000Res, 1000. 24(6). 
299. Cao, Q., et al., Reconstruction of enhancer-target networks in 935 samples of human primary cells, tissues and cell lines. Nat Genet, 2017. 49(10): p. 1428-1436.

300. Xie, W., et al., RNF40 regulates gene expression in an epigenetic context-dependent manner. Genome Biol, 2017. 18(1): p. 32.

301. Fontanals-Cirera, B., et al., Harnessing BET Inhibitor Sensitivity Reveals AMIGO2 as a Melanoma Survival Gene. Mol Cell, 2017. 68(4): p. 731-744.e9.

302. Yokoyama, Y., et al., BET Inhibitors Suppress ALDH Activity by Targeting ALDH1A1 SuperEnhancer in Ovarian Cancer. Cancer Res, 2016. 76(21): p. 6320-6330.

303. Sengupta, D., et al., Disruption of BRD4 at H3K27Ac-enriched enhancer region correlates with decreased c-Myc expression in Merkel cell carcinoma. Epigenetics, 2015. 10(6): p. 460-6.

304. Hamdan, F.H. and S.A. Johnsen, Super enhancers - new analyses and perspectives on the low hanging fruit. Transcription, 2018. 9(2): p. 123-130.

305. Chapuy, B., et al., Discovery and characterization of super-enhancer-associated dependencies in diffuse large B cell lymphoma. Cancer Cell, 2013. 24(6): p. 777-90.

306. Gelato, K.A., et al., Super-enhancers define a proliferative PGC-1alpha-expressing melanoma subgroup sensitive to BET inhibition. Oncogene, 2018. 37(4): p. 512-521.

307. Huang, Y., et al., Molecular and cellular mechanisms of castration resistant prostate cancer. Oncol Lett, 2018. 15(5): p. 6063-6076.

308. Faivre, E.J., et al., Exploitation of Castration-Resistant Prostate Cancer Transcription Factor Dependencies by the Novel BET Inhibitor ABBV-075. Mol Cancer Res, 2017. 15(1): p. 35-44.

309. Muhar, M., et al., SLAM-seq defines direct gene-regulatory functions of the BRD4-MYC axis. Science, 2018.

310. Taniguchi, Y., The Bromodomain and Extra-Terminal Domain (BET) Family: Functional Anatomy of BET Paralogous Proteins. Int J Mol Sci, 2016. 17(11).

311. Lamonica, J.M., et al., Bromodomain protein Brd3 associates with acetylated GATA1 to promote its chromatin occupancy at erythroid target genes. Proc Natl Acad Sci U S A, 2011. 108(22): p. 2.

312. Denis, G.V., et al., Identification of transcription complexes that contain the double bromodomain protein Brd2 and chromatin remodeling machines. J Proteome Res, 2006. 5(3): p. 502-11.

313. Sakamoto, K.M., et al., Protacs: chimeric molecules that target proteins to the Skp1-Cullin-F box complex for ubiquitination and degradation. Proc Natl Acad Sci U S A, 2001. 98(15): p. 8554-9.

314. Saenz, D.T., et al., Novel BET protein proteolysis-targeting chimera exerts superior lethal activity than bromodomain inhibitor (BETi) against post-myeloproliferative neoplasm secondary (s) AML cells. Leukemia, 2017. 31(9): p. 1951-1961. 
315. Mazur, P.K., et al., Combined inhibition of BET family proteins and histone deacetylases as a potential epigenetics-based therapy for pancreatic ductal adenocarcinoma. Nat Med, 2015. 21(10): p. 1163-71.

316. Suraweera, A., K.J. O'Byrne, and D.J. Richard, Combination Therapy With Histone Deacetylase Inhibitors (HDACi) for the Treatment of Cancer: Achieving the Full Therapeutic Potential of HDACi. Front Oncol, 2018. 8: p. 92.

317. Barneda-Zahonero, B. and M. Parra, Histone deacetylases and cancer. Mol Oncol, 2012. 6(6): p. 579-89.

318. Li, Y. and E. Seto, HDACs and HDAC Inhibitors in Cancer Development and Therapy. Cold Spring Harb Perspect Med, 2016. 6(10).

319. Vancurova, I., et al., Combination Therapies Targeting HDAC and IKK in Solid Tumors. Trends Pharmacol Sci, 2018. 39(3): p. 295-306.

320. Chen, Y.J., et al., Novel histone deacetylase inhibitor AR-42 exhibits antitumor activity in pancreatic cancer cells by affecting multiple biochemical pathways. PLoS One, 2017. 12(8): p. e0183368.

321. He, M., et al., Chidamide Inhibits Aerobic Metabolism to Induce Pancreatic Cancer Cell Growth Arrest by Promoting Mcl-1 Degradation. PLoS One, 2016. 11(11): p. e0166896.

322. Dalla Pozza, E., et al., Trichostatin A alters cytoskeleton and energy metabolism of pancreatic adenocarcinoma cells: An in depth proteomic study. J Cell Biochem, 2018. 119(3): p. 2696-2707.

323. Wang, G., et al., Class I and class II histone deacetylases are potential therapeutic targets for treating pancreatic cancer. PLoS One, 2012. 7(12): p. e52095.

324. Cai, M.H., et al., Depletion of HDAC1, 7 and 8 by Histone Deacetylase Inhibition Confers Elimination of Pancreatic Cancer Stem Cells in Combination with Gemcitabine. Sci Rep, 2018. 8(1): p. 1621.

325. Chan, E., et al., Phase I/II study of mocetinostat in combination with gemcitabine for patients with advanced pancreatic cancer and other advanced solid tumors. Cancer Chemother Pharmacol, 2018. 81(2): p. 355-364.

326. Samulitis, B.K., et al., Gemcitabine resistant pancreatic cancer cell lines acquire an invasive phenotype with collateral hypersensitivity to histone deacetylase inhibitors. Cancer Biol Ther, 2015. 16(1): p. 43-51.

327. Pan, Y., et al., Gd-Metallofullerenol Nanomaterial Suppresses Pancreatic Cancer Metastasis by Inhibiting the Interaction of Histone Deacetylase 1 and Metastasis-Associated Protein 1. ACS Nano, 2015. 9(7): p. 6826-36.

328. Meidhof, S., et al., ZEB1-associated drug resistance in cancer cells is reversed by the class I HDAC inhibitor mocetinostat. EMBO Mol Med, 2015. 7(6): p. 831-47. 
329. Aghdassi, A., et al., Recruitment of histone deacetylases HDAC1 and HDAC2 by the transcriptional repressor ZEB1 downregulates E-cadherin expression in pancreatic cancer. Gut, 2012. 61(3): p. 439-48.

330. Mishra, V.K., et al., Histone deacetylase class-I inhibition promotes epithelial gene expression in pancreatic cancer cells in a BRD4- and MYC-dependent manner. Nucleic Acids Res, 2017. 45(11): p. 6334-6349.

331. Sanchez, G.J., et al., Genome-wide dose-dependent inhibition of histone deacetylases studies reveal their roles in enhancer remodeling and suppression of oncogenic super-enhancers. Nucleic Acids Res, 2018. 46(4): p. 1756-1776.

332. Huang, J.P. and K. Ling, EZH2 and histone deacetylase inhibitors induce apoptosis in triple negative breast cancer cells by differentially increasing H3 Lys(27) acetylation in the BIM gene promoter and enhancers. Oncol Lett, 2017. 14(5): p. 5735-5742.

333. Greer, C.B., et al., Histone Deacetylases Positively Regulate Transcription through the Elongation Machinery. Cell Rep, 2015. 13(7): p. 1444-1455.

334. Tao, R., et al., HDAC-mediated deacetylation of KLF5 associates with its proteasomal degradation. Biochem Biophys Res Commun, 2018.

335. Wang, L., et al., Histone/protein deacetylase inhibitor therapy for enhancement of Foxp3+ Tregulatory cell function posttransplantation. Am J Transplant, 2018.

336. Kohler, S. and L.A. Cirillo, Stable chromatin binding prevents FoxA acetylation, preserving FoxA chromatin remodeling. J Biol Chem, 2010. 285(1): p. 464-72.

337. Diaferia, G.R., et al., Dissection of transcriptional and cis-regulatory control of differentiation in human pancreatic cancer. Embo J, 2016. 35(6): p. 595-617.

338. Sainsbury, S., C. Bernecky, and P. Cramer, Structural basis of transcription initiation by RNA polymerase II. Nat Rev Mol Cell Biol, 2015. 16(3): p. 129-43.

339. An integrated encyclopedia of DNA elements in the human genome. Nature, 2012. 489(7414): $\mathrm{p}$. 57-74.

340. Gotze, S., et al., Histone deacetylase inhibitors induce attenuation of Wnt signaling and TCF7L2 depletion in colorectal carcinoma cells. Int J Oncol, 2014. 45(4): p. 1715-23.

341. Yu, Q., et al., Long non-coding RNA CCAT1 that can be activated by c-Myc promotes pancreatic cancer cell proliferation and migration. Am J Transl Res, 2016. 8(12): p. 5444-5454.

342. Taberlay, P.C., et al., Three-dimensional disorganization of the cancer genome occurs coincident with long-range genetic and epigenetic alterations. Genome Res, 2016. 26(6): p. 719-31.

343. Shukla, S., et al., Aberrant Activation of a Gastrointestinal Transcriptional Circuit in Prostate Cancer Mediates Castration Resistance. Cancer Cell, 2017. 32(6): p. 792-806.e7.

344. Walsh, A.L., et al., Long noncoding RNAs and prostate carcinogenesis: the missing 'linc'? Trends Mol Med, 2014. 20(8): p. 428-36. 
345. Zeng, C., et al., Overexpression of the long non-coding RNA PVT1 is correlated with leukemic cell proliferation in acute promyelocytic leukemia. J Hematol Oncol, 2015. 8(126): p. 015-0223.

346. Rathert, P., et al., Transcriptional plasticity promotes primary and acquired resistance to BET inhibition. Nature, 2015. 525(7570): p. 543-547.

347. Ganesan, A., Epigenetics: the first 25 centuries. Philos Trans R Soc Lond B Biol Sci, 2018. 373(1748).

348. Lin, X., et al. ABBV-744, a first-in-class and highly selective inhibitor of the second beomodomain of BET family proteins, displays robust activities in preclinical models of acute myelogenous leukemia. in AACR Annual Meeting 2018.

349. Peng, X.L., et al., MyoD- and FoxO3-mediated hotspot interaction orchestrates super-enhancer activity during myogenic differentiation. Nucleic Acids Res, 2017. 45(15): p. 8785-8805.

350. Hay, D., et al., Genetic dissection of the alpha-globin super-enhancer in vivo. Nat Genet, 2016. 48(8): p. 895-903.

351. Shin, H.Y., et al., Hierarchy within the mammary STAT5-driven Wap super-enhancer. Nat Genet, 2016. 48(8): p. 904-911.

352. Zhang, Q., et al., Hypoxia-inducible factor-2alpha promotes tumor progression and has crosstalk with Wnt/beta-catenin signaling in pancreatic cancer. Mol Cancer, 2017. 16(1): p. 119.

353. Zhong, Y., et al., Mutant p53 Together with TGFbeta Signaling Influence Organ-Specific Hematogenous Colonization Patterns of Pancreatic Cancer. Clin Cancer Res, 2017. 23(6): p. 1607-1620.

354. Nomura, A., et al., Inhibition of NF-kappa B pathway leads to deregulation of epithelialmesenchymal transition and neural invasion in pancreatic cancer. Lab Invest, 2016. 96(12): p. 1268-1278.

355. Nhili, R., et al., Targeting the DNA-binding activity of the human ERG transcription factor using new heterocyclic dithiophene diamidines. Nucleic Acids Res, 2013. 41(1): p. 125-38.

356. Huang, M., et al., The suppression of bromodomain and extra-terminal domain inhibits vascular inflammation by blocking NF-kappaB and MAPK activation. Br J Pharmacol, 2017. 174(1): p. 101115.

357. Hensel, T., et al., Targeting the EWS-ETS transcriptional program by BET bromodomain inhibition in Ewing sarcoma. Oncotarget, 2016. 7(2): p. 1451-63.

358. Mertz, J.A., et al. Pharmacodynamic assessment in whole blood for the BET bromodomain inhibitor CPI-0610 of target engagement in patients with progressive lymphoma. in AACR Annual Meeting 2018. 2018.

359. Langmead, B. and S.L. Salzberg, Fast gapped-read alignment with Bowtie 2. Nat Methods, 2012. 9(4): p. 357-9. 
360. Li, H., et al., The Sequence Alignment/Map format and SAMtools. Bioinformatics, 2009. 25(16): $\mathrm{p}$. 2078-9.

361. Ramirez, F., et al., deepTools: a flexible platform for exploring deep-sequencing data. Nucleic Acids Res, 2014. 42(Web Server issue): p. 5.

362. Robinson, J.T., et al., Integrative genomics viewer. Nat Biotechnol. 2011 Jan;29(1):24-6. doi: 10.1038/nbt.1754.

363. Thorvaldsdottir, H., J.T. Robinson, and J.P. Mesirov, Integrative Genomics Viewer (IGV): highperformance genomics data visualization and exploration. Brief Bioinform, 2013. 14(2): p. 178-92.

364. Zhao, L., et al., Molecular subtyping of cancer: current status and moving toward clinical applications. Brief Bioinform, 2018. 12(4969517).

365. Dai, X., et al., Breast cancer intrinsic subtype classification, clinical use and future trends. Am J Cancer Res, 2015. 5(10): p. 2929-43.

366. Andre, F. and L. Pusztai, Molecular classification of breast cancer: implications for selection of adjuvant chemotherapy. Nat Clin Pract Oncol, 2006. 3(11): p. 621-32.

367. Comprehensive molecular characterization of human colon and rectal cancer. Nature, 2012. 487(7407): p. 330-7.

368. Integrated Genomic Characterization of Pancreatic Ductal Adenocarcinoma. Cancer Cell, 2017. 32(2): p. 185-203.

369. Nylander, K., et al., Differential expression of p63 isoforms in normal tissues and neoplastic cells. J Pathol, 2002. 198(4): p. 417-27.

370. Koike, M., et al., Molecular detection of circulating esophageal squamous cell cancer cells in the peripheral blood. Clin Cancer Res, 2002. 8(9): p. 2879-82.

371. Ye, S., et al., p63 regulates growth of esophageal squamous carcinoma cells via the Akt signaling pathway. Int J Oncol, 2014. 44(6): p. 2153-9.

372. Kakuki, T., et al., Dysregulation of junctional adhesion molecule-A via p63/GATA-3 in head and neck squamous cell carcinoma. Oncotarget, 2016. 7(23): p. 33887-900.

373. Bir, F., et al., Potential utility of p63 expression in differential diagnosis of non-small-cell lung carcinoma and its effect on prognosis of the disease. Med Sci Monit, 2014. 20: p. 219-26.

374. Lo lacono, M., et al., p63 and p73 isoform expression in non-small cell lung cancer and corresponding morphological normal lung tissue. J Thorac Oncol, 2011. 6(3): p. 473-81.

375. Kouwenhoven, E.N., et al., Transcription factor 063 bookmarks and regulates dynamic enhancers during epidermal differentiation. EMBO Rep, 2015. 16(7): p. 863-78.

376. Xie, W., et al., RNF40 regulates gene expression in an epigenetic context-dependent manner. Genome Biol, 2017. 18(1): p. 017-1159. 
377. Starruss, J., et al., Morpheus: a user-friendly modeling environment for multiscale and multicellular systems biology. Bioinformatics, 2014. 30(9): p. 1331-2.

378. Bruns, C.J., et al., In vivo selection and characterization of metastatic variants from human pancreatic adenocarcinoma by using orthotopic implantation in nude mice. Neoplasia, 1999. 1(1): p. 50-62.

379. Nakamura, T., I.J. Fidler, and K.R. Coombes, Gene expression profile of metastatic human pancreatic cancer cells depends on the organ microenvironment. Cancer Res, 2007. 67(1): p. $139-48$.

380. Soucie, E.L., et al., Lineage-specific enhancers activate self-renewal genes in macrophages and embryonic stem cells. Science, 2016. 351(6274): p. 21.

381. Maherali, N., et al., Directly reprogrammed fibroblasts show global epigenetic remodeling and widespread tissue contribution. Cell Stem Cell, 2007. 1(1): p. 55-70.

382. Simandi, Z., et al., OCT4 Acts as an Integrator of Pluripotency and Signal-Induced Differentiation. Mol Cell, 2016. 63(4): p. 647-661.

383. $\mathrm{Xu}$, J., et al., Transcriptional competence and the active marking of tissue-specific enhancers by defined transcription factors in embryonic and induced pluripotent stem cells. Genes Dev, 2009. 23(24): p. 2824-38.

384. Ye, L.Y., et al., Hypoxia-inducible factor 1alpha expression and its clinical significance in pancreatic cancer: a meta-analysis. Pancreatology, 2014. 14(5): p. 391-7.

385. Matsuo, Y., et al., Hypoxia inducible factor-1 alpha plays a pivotal role in hepatic metastasis of pancreatic cancer: an immunohistochemical study. J Hepatobiliary Pancreat Sci, 2014. 21(2): p. 105-12.

386. Shi, C.Y., et al., HIF1 contributes to hypoxia-induced pancreatic cancer cells invasion via promoting QSOX1 expression. Cell Physiol Biochem, 2013. 32(3): p. 561-8.

387. Yu, F., et al., The transcription factor Bhlhe40 is a switch of inflammatory versus antiinflammatory Th1 cell fate determination. J Exp Med, 2018. 215(7): p. 1813-1821.

388. Hamilton, K.A., et al., Mice lacking the transcriptional regulator Bh/he40 have enhanced neuronal excitability and impaired synaptic plasticity in the hippocampus. PLoS One, 2018. 13(5).

389. Halstead, A.M., et al., Bladder-cancer-associated mutations in RXRA activate peroxisome proliferator-activated receptors to drive urothelial proliferation. Elife, 2017. 16(6): p. 30862.

390. Yin, J., et al., Genetic variants in the vitamin $D$ pathway genes VDBP and RXRA modulate cutaneous melanoma disease-specific survival. Pigment Cell Melanoma Res, 2016. 29(2): p. 17685.

391. Prenzel, T., et al., Estrogen-dependent gene transcription in human breast cancer cells relies upon proteasome-dependent monoubiquitination of histone H2B. Cancer Res, 2011. 71(17): p. 5739-53. 
392. Buenrostro, J.D., et al., ATAC-seq: A Method for Assaying Chromatin Accessibility GenomeWide. Curr Protoc Mol Biol, 2015. 109(21): p. 1-9.

393. Zhang, Y., et al., Model-based analysis of ChIP-Seq (MACS). Genome Biol, 2008. 9(9): p. 20089.

394. Ye, T., et al., seqMINER: an integrated ChIP-seq data interpretation platform. Nucleic Acids Res, 2011. 39(6): p. 21.

395. Ye, T., et al., Interpreting and visualizing ChIP-seq data with the seqMINER software. Methods Mol Biol, 2014: p. 0512-6_8.

396. Ross-Innes, C.S., et al., Differential oestrogen receptor binding is associated with clinical outcome in breast cancer. Nature, 2012. 481(7381): p. 389-93.

397. Afgan, E., et al., The Galaxy platform for accessible, reproducible and collaborative biomedical analyses: 2016 update. Nucleic Acids Res, 2016. 44(W1): p. W3-W10.

398. Madani Tonekaboni, S.A., et al., CREAM: Clustering of genomic REgions Analysis Method. bioRxiv, 2017.

399. Quinlan, A.R. and I.M. Hall, BEDTools: a flexible suite of utilities for comparing genomic features. Bioinformatics, 2010. 26(6): p. 841-2.

400. Chen, H. and P.C. Boutros, VennDiagram: a package for the generation of highly-customizable Venn and Euler diagrams in R. BMC Bioinformatics, 2011. 12(35): p. 1471-2105.

401. Kuleshov, M.V., et al., Enrichr: a comprehensive gene set enrichment analysis web server 2016 update. Nucleic Acids Res, 2016. 44(W1): p. 3.

402. Griffon, A., et al., Integrative analysis of public ChIP-seq experiments reveals a complex multi-cell regulatory landscape. Nucleic Acids Res, 2015. 43(4): p. 3.

403. Cheneby, J., et al., ReMap 2018: an updated atlas of regulatory regions from an integrative analysis of DNA-binding ChIP-seq experiments. Nucleic Acids Res, 2018. 46(D1): p. D267-D275.

404. Karolchik, D., et al., The UCSC Table Browser data retrieval tool. Nucleic Acids Res, 2004. 32(Database issue).

405. Trapnell, C., L. Pachter, and S.L. Salzberg, TopHat: discovering splice junctions with RNA-Seq. Bioinformatics, 2009. 25(9): p. 1105-11.

406. Trapnell, C., et al., Differential gene and transcript expression analysis of RNA-seq experiments with TopHat and Cufflinks. Nat Protoc, 2012. 7(3): p. 562-78.

407. de Hoon, M.J., et al., Open source clustering software. Bioinformatics, 2004. 20(9): p. 1453-4.

408. Saldanha, A.J., Java Treeview--extensible visualization of microarray data. Bioinformatics, 2004. 20(17): p. 3246-8. 
409. Subramanian, A., et al., Gene set enrichment analysis: a knowledge-based approach for interpreting genome-wide expression profiles. Proc Natl Acad Sci U S A, 2005. 102(43): p. $15545-50$.

410. Shannon, P., et al., Cytoscape: a software environment for integrated models of biomolecular interaction networks. Genome Res, 2003. 13(11): p. 2498-504.

411. Janky, R., et al., iRegulon: from a gene list to a gene regulatory network using large motif and track collections. PLoS Comput Biol, 2014. 10(7).

412. Tian, B., et al., CDK9-dependent transcriptional elongation in the innate interferon-stimulated gene response to respiratory syncytial virus infection in airway epithelial cells. J Virol, 2013. 87(12): p. 7075-92.

413. Scheel, A.H., et al., Immunofluorescence-based screening identifies germ cell associated microRNA 302 as an antagonist to p63 expression. Cell Cycle, 2009. 8(9): p. 1426-32.

414. Kim, V.M. and N. Ahuja, Early detection of pancreatic cancer. Chinese Journal of Cancer Research, 2015. 27(4): p. 321-331.

415. Rahib, L., et al., Projecting cancer incidence and deaths to 2030: the unexpected burden of thyroid, liver, and pancreas cancers in the United States. Cancer Res, 2014. 74(11): p. 2913-21.

416. Li, X., et al., MYC-mediated synthetic lethality for treatment of hematological malignancies. Curr Cancer Drug Targets, 2015. 15(1): p. 53-70.

417. Houzelstein, D., et al., Growth and early postimplantation defects in mice deficient for the bromodomain-containing protein Brd4. Mol Cell Biol, 2002. 22(11): p. 3794-802.

418. Wali, V.B., et al., Systematic Drug Screening Identifies Tractable Targeted Combination Therapies in Triple-Negative Breast Cancer. Cancer Res, 2017. 77(2): p. 566-578.

419. Horwitz, S.B., Taxol (paclitaxel): mechanisms of action. Annals of oncology : official journal of the European Society for Medical Oncology, 1994. 5 Suppl 6: p. S3-6.

420. Pusztai, L., et al., Changes in plasma levels of inflammatory cytokines in response to paclitaxel chemotherapy. Cytokine, 2004. 25(3): p. 94-102.

421. Duffy, C.P., et al., Enhancement of chemotherapeutic drug toxicity to human tumour cells in vitro by a subset of non-steroidal anti-inflammatory drugs (NSAIDs). European Journal of Cancer, 1998. 34(8): p. 1250-1259.

422. Lamendola, D.E., et al., Molecular Description of Evolving Paclitaxel Resistance in the SKOV-3 Human Ovarian Carcinoma Cell Line. Cancer Research, 2003. 63(9): p. 2200.

423. Kim, K.H., et al., NSAID-activated gene 1 mediates pro-inflammatory signaling activation and paclitaxel chemoresistance in type I human epithelial ovarian cancer stem-like cells. Oncotarget, 2016. 7(44): p. 72148-72166.

424. Kelly, M.G., et al., TLR-4 Signaling Promotes Tumor Growth and Paclitaxel Chemoresistance in Ovarian Cancer. Cancer Research, 2006. 66(7): p. 3859. 
425. Tu, S.H., et al., Garcinol sensitizes breast cancer cells to Taxol through the suppression of caspase-3/iPLA2 and NF-kappaB/Twist1 signaling pathways in a mouse $4 T 1$ breast tumor model. Food Funct, 2017. 8(3): p. 1067-1079.

426. Zhao, X., et al., Inhibiting tumor necrosis factor-alpha diminishes desmoplasia and inflammation to overcome chemoresistance in pancreatic ductal adenocarcinoma. Oncotarget, 2016. 7(49): p. 81110-81122.

427. Pear, W., et al., Modular domains within a super enhancer determine drug resistance in T-cell acute lymphoblastic leukemia. Experimental Hematology, 2016. 44(9): p. S25.

428. Doroshow, D.B., J.P. Eder, and P.M. LoRusso, BET inhibitors: a novel epigenetic approach. Ann Oncol, 2017. 28(8): p. 1776-1787.

429. Dombret, H., et al., A Phase 1 Study of the BET-Bromodomain Inhibitor OTX015 in Patients with Advanced Acute Leukemia. Blood, 2014. 124(21): p. 117.

430. Shan, X., et al., Replication Study: Inhibition of BET recruitment to chromatin as an effective treatment for MLL-fusion leukaemia. Elife, 2017. 6.

431. Bernasconi, E., et al., Preclinical evaluation of the BET bromodomain inhibitor BAY 1238097 for the treatment of lymphoma. Br J Haematol, 2017. 178(6): p. 936-948.

432. Pawar, A., et al., Resistance to BET Inhibitor Leads to Alternative Therapeutic Vulnerabilities in Castration-Resistant Prostate Cancer. Cell Rep, 2018. 22(9): p. 2236-2245.

433. Harris, R.S., S.K. Petersen-Mahrt, and M.S. Neuberger, RNA editing enzyme APOBEC1 and some of its homologs can act as DNA mutators. Mol Cell, 2002. 10(5): p. 1247-53.

434. Pauklin, S., et al., Alternative induction of meiotic recombination from single-base lesions of DNA deaminases. Genetics, 2009. 182(1): p. 41-54.

435. Fry, S.A., et al., Cadherin-5: a biomarker for metastatic breast cancer with optimum efficacy in oestrogen receptor-positive breast cancers with vascular invasion. $\mathrm{Br} \mathrm{J}$ Cancer, 2016. 114(9): $\mathrm{p}$. 1019-26.

436. Liang, W., et al., CAV-1 contributes to bladder cancer progression by inducing epithelial-tomesenchymal transition. Urol Oncol, 2014. 32(6): p. 855-63.

437. Luan, T.Y., et al., Expression of caveolin-1 is correlated with lung adenocarcinoma proliferation, migration, and invasion. Med Oncol, 2015. 32(7): p. 207.

438. Tam, K.J., et al., Semaphorin 3 C drives epithelial-to-mesenchymal transition, invasiveness, and stem-like characteristics in prostate cells. Sci Rep, 2017. 7(1): p. 11501.

439. Gollavilli, P.N., et al., EWS/ETS-Driven Ewing Sarcoma Requires BET Bromodomain Proteins. Cancer Res, 2018. 78(16): p. 4760-4773.

440. Pirngruber, J., et al., CDK9 directs $H 2 B$ monoubiquitination and controls replication-dependent histone mRNA 3'-end processing. EMBO Rep, 2009. 10(8): p. 894-900. 
441. Shema, E., et al., The histone H2B-specific ubiquitin ligase RNF20/hBRE1 acts as a putative tumor suppressor through selective regulation of gene expression. Genes Dev, 2008. 22(19): $p$. 2664-76.

442. Pirngruber, J. and S.A. Johnsen, Induced G1 cell-cycle arrest controls replication-dependent histone mRNA 3' end processing through p21, NPAT and CDK9. Oncogene, 2010. 29(19): p. 2853-63.

443. Sun, B., et al., Synergistic activity of BET protein antagonist-based combinations in mantle cell lymphoma cells sensitive or resistant to ibrutinib. Blood, 2015. 126(13): p. 1565-74.

444. Stewart, H.J.S., et al., BET Inhibition Suppresses S100A8 and S100A9 Expression in Acute Myeloid Leukemia Cells and Synergises with Daunorubicin in Causing Cell Death. Bone Marrow Res, 2018. 2018: p. 5742954.

445. Xie, F., et al., The BET inhibitor I-BET762 inhibits pancreatic ductal adenocarcinoma cell proliferation and enhances the therapeutic effect of gemcitabine. Sci Rep, 2018. 8(1): p. 8102.

446. Zanellato, I., D. Colangelo, and D. Osella, JQ1, a BET inhibitor, synergizes with cisplatin and induces apoptosis in highly chemoresistant malignant pleural mesothelioma cells. Curr Cancer Drug Targets, 2017.

447. Vazquez, R., et al., The bromodomain inhibitor OTX015 (MK-8628) exerts anti-tumor activity in triple-negative breast cancer models as single agent and in combination with everolimus. Oncotarget, 2017. 8(5): p. 7598-7613.

448. Liu, P.Y., et al., The BET bromodomain inhibitor exerts the most potent synergistic anticancer effects with quinone-containing compounds and anti-microtubule drugs. Oncotarget, 2016. 7(48): p. 79217-79232.

449. Huang, W., et al., Selective inhibition of BET proteins reduces pancreatic damage and systemic inflammation in bile acid- and fatty acid ethyl ester- but not caerulein-induced acute pancreatitis. Pancreatology, 2017. 17(5): p. 689-697.

450. Duan, Q., et al., BET bromodomain inhibition suppresses innate inflammatory and profibrotic transcriptional networks in heart failure. Sci Transl Med, 2017. 9(390).

451. Malhotra, R., et al., Altered regulation and expression of genes by BET family of proteins in COPD patients. PLoS One, 2017. 12(3): p. e0173115.

452. Zhang, Z., et al., Preclinical Efficacy and Molecular Mechanism of Targeting CDK7-Dependent Transcriptional Addiction in Ovarian Cancer. Mol Cancer Ther, 2017. 16(9): p. 1739-1750.

453. Chipumuro, E., et al., CDK7 inhibition suppresses super-enhancer-linked oncogenic transcription in MYCN-driven cancer. Cell, 2014. 159(5): p. 1126-1139.

454. Anders, L., et al., Genome-wide localization of small molecules. Nat Biotechnol, 2014. 32(1): p. 92-6.

455. Ernst, J. and Z. Bar-Joseph, STEM: a tool for the analysis of short time series gene expression data. BMC Bioinformatics, 2006. 7: p. 191-191. 
456. Supek, F., et al., REVIGO summarizes and visualizes long lists of gene ontology terms. PLoS One, 2011. 6(7): p. e21800.

457. Heinz, S., et al., Simple combinations of lineage-determining transcription factors prime cisregulatory elements required for macrophage and B cell identities. Mol Cell, 2010. 38(4): p. 57689.

458. Peeters, Janneke G.C., et al., Inhibition of Super-Enhancer Activity in Autoinflammatory SiteDerived T Cells Reduces Disease-Associated Gene Expression. Cell Reports, 2015. 12(12): p. 1986-1996.

459. Wang, H., et al., Alternative Super-Enhancer States Determine \&lt;em\&gt;MYC\&lt;/em\&gt; Sensitivity to Notch and Brd4 Inhibitors in T Lymphoblastic Leukemia/Lymphoma. Blood, 2014. 124(21): p. 863.

460. Frank, C.L., et al., HDAC inhibitors cause site-specific chromatin remodeling at PU.1-bound enhancers in K562 cells. Epigenetics \& Chromatin, 2016. 9: p. 15.

461. Yohe, M.E., et al., MEK inhibition induces MYOG and remodels super-enhancers in RAS-driven rhabdomyosarcoma. Science Translational Medicine, 2018. 10(448).

462. Heinz, S., et al., The selection and function of cell type-specific enhancers. Nat Rev Mol Cell Biol, 2015. 16(3): p. 144-54.

463. Jiang, Y., et al., Co-activation of super-enhancer-driven CCAT1 by TP63 and SOX2 promotes squamous cancer progression. Nat Commun, 2018. 9(1): p. 3619.

464. Northcott, P.A., et al., Enhancer hijacking activates GFl1 family oncogenes in medulloblastoma. Nature, 2014. 511(7510): p. 428-434.

465. Zawistowski, J.S., et al., Enhancer Remodeling During Adaptive Bypass to MEK Inhibition Is Attenuated by Pharmacological Targeting of the P-TEFb Complex. Cancer Discovery, 2017.

466. Cheng, J.-H., et al., Genome-wide analysis of enhancer RNA in gene regulation across 12 mouse tissues. Scientific Reports, 2015. 5: p. 12648.

467. Zhao, Y., et al., Activation of P-TEFb by Androgen Receptor-Regulated Enhancer RNAs in Castration-Resistant Prostate Cancer. Cell reports, 2016. 15(3): p. 599-610.

468. Kaczkowski, B., et al., Transcriptome analysis of recurrently deregulated genes across multiple cancers identifies new pan-cancer biomarkers. Cancer Research, 2015.

469. Moorthy, S.D., et al., Enhancers and super-enhancers have an equivalent regulatory role in embryonic stem cells through regulation of single or multiple genes. Genome Res, 2017. 27(2): p. 246-258.

470. Jiang, Y.-Y., et al., Targeting super-enhancer-associated oncogenes in oesophageal squamous cell carcinoma. Vol. 66. 2016. gutjnl-2016.

471. Chen, D., et al., Super enhancer inhibitors suppress MYC driven transcriptional amplification and tumor progression in osteosarcoma. Bone Research, 2018. 6(1): p. 11. 
472. Wang, E. and I. Aifantis, Targeting the Noncoding Genome: Superenhancers Meet Their Kryptonite. Cancer discovery, 2017. 7(10): p. 1065-1066.

473. Parker, S.C.J., et al., Chromatin stretch enhancer states drive cell-specific gene regulation and harbor human disease risk variants. Proceedings of the National Academy of Sciences, 2013. 110(44): p. 17921.

474. Quang, D.X., et al., Motif signatures in stretch enhancers are enriched for disease-associated genetic variants. Epigenetics Chromatin, 2015. 8: p. 23.

475. Khan, A., A. Mathelier, and X. Zhang, Super-enhancers are transcriptionally more active and cell type-specific than stretch enhancers. Epigenetics, 2018.

476. Hong, J.-W., D.A. Hendrix, and M.S. Levine, Shadow Enhancers as a Source of Evolutionary Novelty. Science (New York, N.Y.), 2008. 321(5894): p. 1314-1314.

477. Cao, K., et al., SET1A/COMPASS and shadow enhancers in the regulation of homeotic gene expression. Genes \& Development, 2017. 31(8): p. 787-801.

478. Zhao, H.X., et al., Rapid and efficient reprogramming of human amnion-derived cells into pluripotency by three factors OCT4/SOX2/NANOG. Differentiation, 2010. 80(2-3): p. 123-9.

479. Li, Y., et al., Generation of human-induced pluripotent stem cells from gut mesentery-derived cells by ectopic expression of OCT4/SOX2/NANOG. Cell Reprogram, 2010. 12(3): p. 237-47.

480. Kim, J., et al., An extended transcriptional network for pluripotency of embryonic stem cells. Cell, 2008. 132(6): p. 1049-61.

481. Kaushal, S., et al., Activation of the myogenic lineage by MEF2A, a factor that induces and cooperates with MyoD. Science, 1994. 266(5188): p. 1236-40.

482. Naidu, P.S., et al., Myogenin and MEF2 function synergistically to activate the MRF4 promoter during myogenesis. Mol Cell Biol, 1995. 15(5): p. 2707-18.

483. Neph, S., et al., Circuitry and dynamics of human transcription factor regulatory networks. Cell, 2012. 150(6): p. 1274-1286.

484. Hagenbuchner, J. and M.J. Ausserlechner, Targeting transcription factors by small compoundsCurrent strategies and future implications. Biochemical Pharmacology, 2016. 107: p. 1-13.

485. Bhagwat, A.S. and C.R. Vakoc, Targeting Transcription Factors in Cancer. Trends in cancer, 2015. 1(1): p. 53-65.

486. de-Leon, S.B.-T., Perturbation analysis analyzed - Mathematical modeling of intact and perturbed gene regulatory circuits for animal development. Developmental biology, 2010. 344(2): p. 11101118.

487. Yu, T., B. Tang, and X. Sun, Development of Inhibitors Targeting Hypoxia-Inducible Factor 1 and 2 for Cancer Therapy. Yonsei Medical Journal, 2017. 58(3): p. 489-496. 
488. Vakonaki, E., et al., Overexpression and ratio disruption of DeltaNp63 and TAp63 isoform equilibrium in endometrial adenocarcinoma: correlation with obesity, menopause, and grade I/II tumors. J Cancer Res Clin Oncol, 2012. 138(8): p. 1271-8.

489. Yan, W., Y. Zhang, and X. Chen, TAp63y and $\triangle N p 63 y$ are regulated by RBM38 via mRNA stability and have an opposing function in growth suppression. Oncotarget, 2017. 8(45): p. 7832778339.

490. Yoshida, M., et al., Development of an integrated CRISPRi targeting DeltaNp63 for treatment of squamous cell carcinoma. Oncotarget, 2018. 9(49): p. 29220-29232.

491. Kajiwara, C., et al., p63-dependent Dickkopf3 expression promotes esophageal cancer cell proliferation via CKAP4. Cancer Res, 2018.

492. Hoebe, E., et al., Epstein-Barr Virus Gene BARF1 Expression is Regulated by the Epithelial Differentiation Factor DeltaNp63alpha in Undifferentiated Nasopharyngeal Carcinoma. Cancers (Basel), 2018. 10(3).

493. Mollo, M.R., et al., p63-dependent and independent mechanisms of nectin-1 and nectin-4 regulation in the epidermis. Experimental Dermatology, 2015. 24(2): p. 114-119.

494. Yang, K., et al., DeltaNp63alpha Transcriptionally Regulates the Expression of CTEN That Is Associated with Prostate Cell Adhesion. PLoS One, 2016. 11(1): p. e0147542.

495. Holcakova, J., et al., DeltaNp63 activates EGFR signaling to induce loss of adhesion in triplenegative basal-like breast cancer cells. Breast Cancer Res Treat, 2017. 163(3): p. 475-484.

496. Romano, R.A., et al., An active role of the DeltaN isoform of p63 in regulating basal keratin genes K5 and K14 and directing epidermal cell fate. PLoS One, 2009. 4(5): p. e5623.

497. Lee, H.O., et al., A dominant negative form of p63 inhibits apoptosis in a p53-independent manner. Biochem Biophys Res Commun, 2006. 344(1): p. 166-72.

498. Sethi, I., et al., A global analysis of the complex landscape of isoforms and regulatory networks of p63 in human cells and tissues. BMC Genomics, 2015. 16(1): p. 584.

499. Perez-Pinera, P., et al., RNA-guided gene activation by CRISPR-Cas9-based transcription factors. Nat Methods, 2013. 10(10): p. 973-6.

500. Xu, X., et al., High-fidelity CRISPR/Cas9- based gene-specific hydroxymethylation rescues gene expression and attenuates renal fibrosis. Nature Communications, 2018. 9(1): p. 3509.

501. Deer, E.L., et al., Phenotype and Genotype of Pancreatic Cancer Cell Lines. Pancreas, 2010. 39(4): p. 425-435.

502. Napoli, M., et al., DeltaNp63/DGCR8-Dependent MicroRNAs Mediate Therapeutic Efficacy of HDAC Inhibitors in Cancer. Cancer Cell, 2016. 29(6): p. 874-888.

503. Sheppard, G.S., et al. Discovery of ABBV-744, a first-in-class highly BDIl-selective BET bromodomain inhibitor. in American Association for Cancer Research Annual Meeting 2018. 2018. 
504. DeMars, K.M., et al., Selective degradation of BET proteins with $d B E T 1$, a proteolysis-targeting chimera, potently reduces pro-inflammatory responses in lipopolysaccharide-activated microglia. Biochem Biophys Res Commun, 2018. 497(1): p. 410-415. 


\section{Abbreviations}

\begin{tabular}{|c|c|}
\hline ac & Acetylation \\
\hline ADEX & Aberrantly Differentiated Endocrine Exocrine \\
\hline ALDH & Aldehyde Dehydrogenase 2 Family \\
\hline AMIGO2 & Adhesion Molecule With Ig Like Domain 2 \\
\hline AML & Acute Myeloid Leukemia \\
\hline APOBEC3C & Apolipoprotein B mRNA Editing Enzyme Catalytic Subunit 3C \\
\hline AR & Androgen Receptor \\
\hline ATAC & Assay for Transposase-Accessible Chromatin \\
\hline BARF1 & BamHI-A Rightward Frame 1 \\
\hline BCL2 & apoptotic regulator B Cell Lymphoma 2 \\
\hline BET & Bromodomain and Extraterminal \\
\hline $\mathrm{BETi}$ & small molecule BET inhibitors \\
\hline BGP & $\beta$-glycerophosphate disodium salt hydrate \\
\hline BHLHE40 & Basic Helix-Loop-Helix Family Member E40 \\
\hline BIM & B cell lymphoma-2-like 11 \\
\hline BRD2 & Bromodomain containing 2 \\
\hline BRD3 & Bromodomain containing 3 \\
\hline BRD4 & Bromodomain containing 4 \\
\hline BSA & Bovine Serum Albumin \\
\hline CAV1 & Caveolin 1 \\
\hline CBP & CREB-binding protein \\
\hline CCAT1 & Colon Cancer Associated Transcript 1 \\
\hline CDH1 & Calcium-dependent adhesion protein-1 \\
\hline CDH5 & Cadherin 5 \\
\hline CDK7 & Cyclin Dependent Kinase 7 \\
\hline CDK8 & Cyclin dependent kinase 8 \\
\hline CDKN2A & Cyclin dependent kinase inhibitor $2 \mathrm{~A}$ \\
\hline cDNA & Complementary DNA \\
\hline ChEA & ChIP Enrichment Analysis \\
\hline ChIP-seq & Chromatin immunoprecipitation followed by high throughput sequencing \\
\hline ChRO-seq & Chromatin run-on and sequencing \\
\hline CORES & Clusters Of cis-Regulatory Elements \\
\hline
\end{tabular}




\begin{tabular}{|c|c|}
\hline CREAM & Clustering of genomic Regions Analysis Method \\
\hline CTCF & CCTC-Binding Factor \\
\hline deltaNp63 & Transactivation domain-lacking p63 isoform \\
\hline dNTPs & deoxyribonucleotides \\
\hline DSIF & DRB-sensitivity inducing factor \\
\hline E2 & Estradiol \\
\hline EDTA & Ethylenediamine tetraacetic acid \\
\hline EGFR & Epidermal growth factor receptor \\
\hline EGTA & ethylene glycol tetraacetic acid \\
\hline EHF & ETS Homologous Factor \\
\hline EMT & Epithelial-to-mesenchymal transition \\
\hline ENCODE & Encyclopedia of DNA Elements \\
\hline EPAS1, HIF2A & Endothelial PAS Domain Protein 1 \\
\hline ER & Estrogen Receptor \\
\hline ER+ & Estrogen Receptor positive \\
\hline EREs & Estrogen Eesponse Elements \\
\hline eRNA & Enhancer ribonucleic acids \\
\hline ERa & Estrogen receptor-alpha \\
\hline ETS & E26 transformation-specific \\
\hline EVI1 & Ecotropic virus integration site-1 \\
\hline EZH2 & Enhancer of Zeste 2 \\
\hline FAT2 & FAT Atypical Cadherin 2 \\
\hline FOXA1 & Forkhead Box A1 \\
\hline FOXA2 & Forkhead Box A2 \\
\hline FPKM & Fragments per kilobase per million \\
\hline GATA1 & GATA binding protein 1 \\
\hline GATA4 & GATA Binding Protein 4 \\
\hline GATA6 & GATA binding protein 6 \\
\hline GREAT & Genomic Regions Enrichment of Annotations Tool \\
\hline GRO-seq & Global run-on sequencing \\
\hline GSEA & Gene Set Enrichment Analysis \\
\hline H2A & Histone 2A \\
\hline H2Aub1 & Mono-ubiquitination of Histone H2A Lysine 119 \\
\hline H2B & Histone 2B \\
\hline H2Bub1 & Mono-ubiquitination of Histone H2B Lysine 120 \\
\hline H3 & Histone 3 \\
\hline
\end{tabular}




\begin{tabular}{|c|c|}
\hline H3K14ac & Acetylation Histone 3 Lysine 14 \\
\hline H3K18ac & Acetylation Histone 3 Lysine 18 \\
\hline H3K23ac & Acetylation Histone 3 Lysine 23 \\
\hline H3K27ac & Acetylation of Histone 3 Lysine 27 \\
\hline H3K27me3 & Tri-methylation of Histone 3 Lysine 27 \\
\hline H3К36ас & Acetylation Histone 3 Lysine 36 \\
\hline H3K4me1 & Mono-methylation of Histone 3 Lysine 4 \\
\hline H3K4me3 & Tri-methylation of Histone 3 Lysine 4 \\
\hline H3K64me3 & tri-methylation of Histone 3 Lysine 64 \\
\hline H3K9ac & Acetylation Histone 3 Lysine 9 \\
\hline H4 & Histone4 \\
\hline H4K16ac & Acetylation of Histone 4 Lysine 16 \\
\hline H4K20ac & acetylation of Histone 4 Lysine 20 \\
\hline H4K5ac & Acetylation of Histone 4 Lysine 5 \\
\hline H4K8ac & Acetylation of Histone 4 Lysine 8 \\
\hline HAT & Histone acetyltransferase \\
\hline HDACs & Histone deactylases \\
\hline HIF1A & Hypoxia Inducible Factor Alpha Subunit \\
\hline HMTs & Histone methyltransferases \\
\hline IC10 & Inhibitory concentration at $10 \%$ \\
\hline IC25 & Inhibitory concentration at $25 \%$ \\
\hline IC50 & half maximal inhibitory concentration \\
\hline IGV & Integrative Genomics Viewer \\
\hline IKBa & NFKB inhibitor- $\alpha$ \\
\hline $\mathbf{k b}$ & Kilo base \\
\hline KLF4 & Krüppel Like Factor 4 \\
\hline KRAS & KRAS proto-oncogene \\
\hline LCR & Locus control region \\
\hline LIF & Interleukin 6 family cytokine \\
\hline IncRNA & Long non-coding RNA \\
\hline LSD1 & Lysine-specific histone demethylase $1 \mathrm{~A}$ \\
\hline MAPK & Mitogen activated protein kinase \\
\hline me & Methylation \\
\hline me3 & Tri-methylation \\
\hline MECOM & MDS1 and EVI1 complex locus \\
\hline MEF2A & myocyte enhancer factor $2 \mathrm{~A}$ \\
\hline
\end{tabular}




\begin{tabular}{|c|c|}
\hline MEM & minimum essential medium \\
\hline microliter & $\mu l$ \\
\hline micromolar & $\mu \mathrm{M}$ \\
\hline MLL3 & mixed-lineage leukemia 3 \\
\hline MLL4 & mixed-lineage leukemia 4 \\
\hline MSigDB & Molecular signatures database \\
\hline MTD & Maximum tolerable dose \\
\hline MYC & MYC Proto-Oncogene \\
\hline MyoD & myogenic differentiation 1 \\
\hline Nanog Homeobox & NANOG \\
\hline nanomolar & $\mathrm{nM}$ \\
\hline ncRNA & non-coding RNA \\
\hline NECTIN1 & Nectin Cell Adhesion Molecule 1 \\
\hline NELF & Negative elongation factor \\
\hline NF-kB & Nuclear Factor kappa-light-chain-enhancer of activated B cells \\
\hline NIPBL & Nipped-B-Like \\
\hline NSAIDs & Non-steroidal anti-inflammatory drugs \\
\hline NT5 & Non-Targeting \#5 \\
\hline NUT & Nuclear protein in testis \\
\hline p63 & tumor protein p63 \\
\hline p73 & Tumor Protein P73 \\
\hline PacR & Paclitaxel-resistant \\
\hline PanINs & Pancreatic intraepithelial neoplasms \\
\hline PARP & Poly-ADP ribose polymerase \\
\hline PCAT1 & Prostate Cancer Associated Transcript 1 \\
\hline PDAC & Pancreatic ductal adenocarcinoma \\
\hline PDX & Patient-derived xenografts \\
\hline PGC-1 a & PPARG Coactivator $1 \alpha$ \\
\hline PI3K & Phosphatidylinositol 3-kinase \\
\hline PIC & Pre-initiation complex \\
\hline POU5F1 & POU Class 5 Homeobox 1 \\
\hline PPARG & Peroxisome Proliferative Activated Receptor- $\vee$ \\
\hline PRC1 & Polycomb repressive complex 1 \\
\hline PRC2 & Polycomb repressive complex 2 \\
\hline PRMI & Roswell Park Memorial Institute medium \\
\hline PRMTs & Protein arginine methyltransferases \\
\hline
\end{tabular}




\begin{tabular}{|c|c|}
\hline PRO-seq & Precision nuclear run-on sequencing \\
\hline PROTACs & Protein-targeting chimeric molecules \\
\hline PSA & Prostate-specific antigen \\
\hline P-TEFb & Positive elongation factor- $b$ \\
\hline QM & Quasi-mesenchymal \\
\hline qPCR & quantitative real-time PCR \\
\hline RHOV & Ras Homolog Family Member V \\
\hline RNA Pol II & RNA polymerase II \\
\hline RNA sequencing & RNA seq \\
\hline RNF20 & Ring Finger Protein 20 \\
\hline RNF40 & Ring Finger Protein 40 \\
\hline ROSE & Ranking of Super Enhancer \\
\hline RXRA & Retinoid X Receptor Alpha \\
\hline SDS & Sodium Dodecyl Sulfate \\
\hline SEMA3A & Semaphorin $3 A$ \\
\hline SEMA3C & Semaphorin $3 C$ \\
\hline SE & Super Enhancer \\
\hline SLAM-seq & Thiol(SH)-linked alkylation for the metabolic sequencing of RNA \\
\hline SMARCA4 & $\begin{array}{l}\text { SWI/SNF Related, Matrix Associated, Actin Dependent Regulator Of } \\
\text { Chromatin, Subfamily A, Member } 4\end{array}$ \\
\hline SNPs & Single nucleotide polymorphisms \\
\hline SOX2 & SRY-Box 2 \\
\hline STEM & Short Time-series Expression Miner \\
\hline SUV39H1 & Suppressor Of Variegation 3-9 Homolog 1 \\
\hline TADs & Topologically Associating Domains \\
\hline TAp63 & Transactivation domain-containing p63 isoform \\
\hline TCGA & Cancer Genome Atlas \\
\hline TE & Typical enhancer \\
\hline TF & Transcription Factor \\
\hline TGF $\beta$ & Transforming Growth Factor- $\beta$ \\
\hline TMPRSS2 & Transmembrane Protease, Serine 2 \\
\hline TNFAIP3 & TNF Alpha Induced Protein 3 \\
\hline TNFa & Tumor necrosis factor alpha \\
\hline TP53 & Tumor Protein P53 \\
\hline TSS & Transcription Start Site \\
\hline TT-seq & Transient transcriptome sequencing \\
\hline
\end{tabular}


References | Feda Hamdan

\begin{tabular}{cl}
\hline ub & Ubiquitination \\
WAC & WW domain-containing adaptor with coiled-coil \\
ZEB1 & Zinc Finger E-Box Binding Homeobox 1 \\
\hline
\end{tabular}




\section{List of Figures}

Figure 1: Mechanisms of Epigenetic Gene Regulation.............................................................. 6

Figure 2 : The main histone modifications acting as active and repressive marks............................... 9

Figure 3 : Epigenetic writers, erasers, and readers. ................................................................ 11

Figure 4 : Defining histone marks of active, inactive, and poised promoters and enhancers.................. 12

Figure 5 : A schematic diagram depicting the pre-initiation complex at the promoter site. .................... 14

Figure 6 : Transcription factors and enhancers cooperate in differential gene activation. ......................16

Figure 7 : ROSE Algorithm in defining super enhnacers. ........................................................... 19

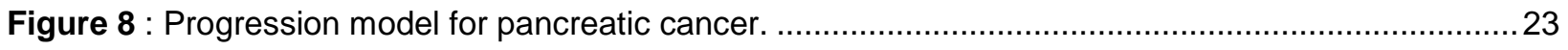

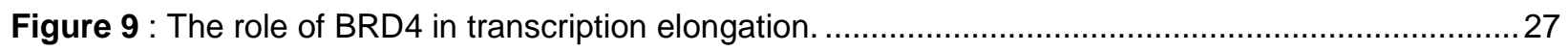

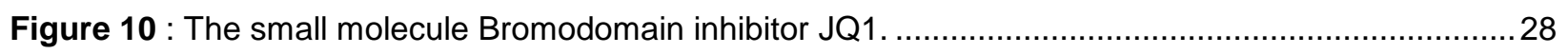

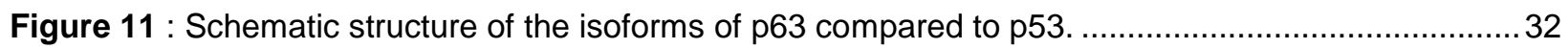

Figure 12 : Different approaches followed to identify super enhancers using the ROSE algorithm.........41

Figure 13 : Tethering of BRD4 to enhancer regions via nuclease-deficient Cas9 (dCas9) can provide causative information about the contribution of individual components of (super) enhancers................. 47

Figure 14 : HDAC inhibition and FOXA1 at distal regulatory elements ............................................. 63

Figure 15 : A schematic model showing distal regulatory mechanisms controlling the expression of the

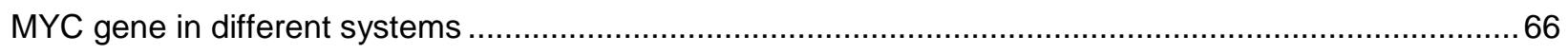

Figure 16 : DeltaNp63 drives gene activation in L3.6pl and BxPC-3.............................................. 78

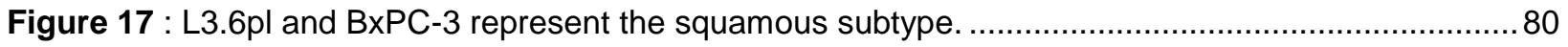

Figure 18 : Depletion of deltaNp63 hampers growth and reverses enrichment of gene expression profiles

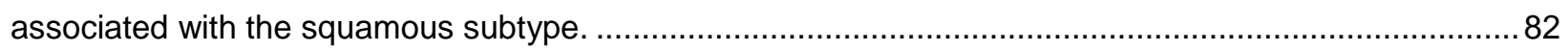

Figure 19 : DeltaNp63 exerts its effects through activation of super enhancers .................................. 86

Figure 20 : Super enhancers in the squamous subtype are highly dependent on deltaNp63 ................88

Figure 21 : DeltaNp63 and its target transcription factors...................................................... 91

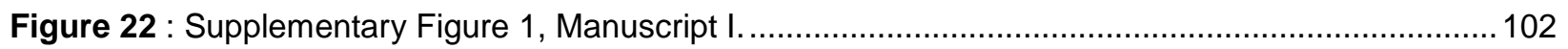




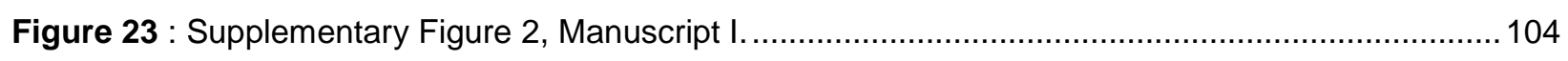

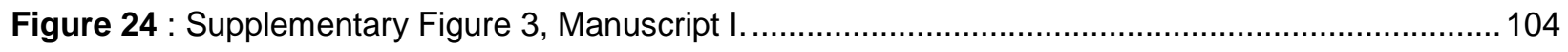

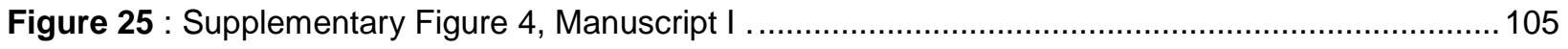

Figure 26 : JQ1 sensitizes L3.6pl cells to paclitaxel ................................................................ 115

Figure 27 : JQ1 reverses transcriptionally activated programs in resistant cells ............................... 117

Figure 28 : BRD4 is gained at putative enhancers near transcriptionally active genes in resistant cells 119

Figure 29 : BET dependent super enhancers are activated in resistant cells................................... 121

Figure 30 : BET-dependent genes respond differentially to low and high doses of JQ1 ..................... 124

Figure 31 : Graphical representation for hypotheses explaining dose-dependent response for BET

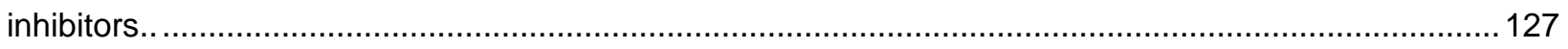

Figure 32 : Positive attributes of enhancers leading them to be good candidates for targeting in cancer. 141

Figure 33 : The transcriptional factor circuitry driven by deltaNp63-dependent super enhancers in the squamous molecular subtype of pancreatic cancer..... 


\section{Acknowledgments}

Doctoral studies is an uphill trip not only due to the difficulty of learning complex concepts and investigating what is so far unknown, but also to the perseverance required to handle failure and falling down countless times and still standing up again and trying without loss of passion and focus. Thus, I would like to thank all the people who made it possible for me to conduct this work and made it fun and worthwhile.

First of all, I would like to thank my doctoral supervisor Professor Steven Johnsen who provided me with the privilege to do my studies in his exceptional lab. I would like to thank him for his patience, limitless support, and extreme generosity with his time, ideas and budget. I would like to thank him for all the 10 minutes meetings that extended to hours, all the collaborations that he has included me into, and all the conferences he sent me to. I would particularly like to thank him for pushing me to a level that I have never thought possible and for believing in me even when I lost belief in myself. I really appreciate the role he played as a super enhancer driving my motivation and passion for everything enhancer-related. I am very thankful for the opportunity to continue working with him and cannot wait to work more, better, and faster on many exciting projects.

Additionally, I would like to thank my thesis committee members, Professor Volker Ellenrieder and Professor Heidi Hahn for all the positive feedback, exciting discussion and support that they provided throughout my PhD years. I would also like to thank Professor Matthias Dobbelstein for providing advice, support, and illuminating conversations during my studies.

Furthermore, I would like to thank the German Academic Exchange Program (DAAD) for providing me with a scholarship that enabled me to pursue my studies in Germany.

I could not have learned all the things I learned without the help of my supporting circuitry of previous and current members of the Johnsen lab. I would like to thank Dr. Florian Wegwitz for dropping anything is his hands to answer a question and help; Dr. Vijayalakshmi Kari for explaining things in the simplest way possible; Dr. Sankari Nagarajan for setting a great example for passion and hard work and teaching me basic lab techniques; Dr. Zeynab Najafova 
for providing good advice and help in technical and scientific aspects; Dr. Vivek Mishra for all the support and fun times; Dr. Robyn Kosinsky for teaching me my first experiment in the lab and providing a great example of organization and efficiency; Jacobe Rapp for all the great times we spent learning together; Nicole Molitor and Sabine Beurmann for excellent technical support; Madhobi Sen for making my second year much easier with her friendship; Geske Schmidt for providing me with the support during my vacation and taking care of my cells; Xin Wang for helping me extensively in starting to work with bioinformatics and providing such great support; Evangelos Prokakis for all the great help and support; Iga Miesczkowska for providing the nice laughs; Ana Patricia Kutschat for her extreme kindness, help and all the fun we had working closely together; Jana Henck for the heart-felt laughs and great support. I would also like to thank all the students I had the chance to supervise and learn from during my studies: Alexandra Michurina; Robert Lorenz Chua; Raquel Vivet Noguer; and Alessandro Consorte.

My thanks extend to all the members of the general, visceral and pediatric surgery in the University Medical Center in Göttingen with special thanks to Jessica Eggert.

I would also like to thank all the people I had the chance to discuss science and ideas with and learn from in collaborating labs: Dr. Med. Oliver Hahn, Dr. Maria Wiese; PD. Dr. Elisabeth Hessmann; Anusha Sriraman; Sabrina Rodewald; Melanie Patzak; Marie Hasselhuhn; Zhe Zhang; and Shilpa Patil.

I would like to thank the people who took from their time to proofread my thesis: Robyn Kosinsky, Ana Kutschat, Geske Schmidt, and Anna Dyas.

I would also like to thank all my friends in Jordan and Germany for the unwavering support they provided me during my studies. Most importantly, I would like to thank my family who pushed me to strive to work as hard as I can and enabled me to pursue my dreams with their great support. 
Curriculum Vitae | Feda Hamdan 